Interpersonal cross border knowledge transfer within the multinational enterprise: Subsidiary power, intra-MNE competition and cultural intelligence

By

\title{
Himadree Phookan
}

\author{
A thesis submitted to the \\ Victoria University of Wellington \\ in fulfilment of the requirements for the degree of \\ Doctor of Philosophy in International Business
}

Victoria University of Wellington 2020 


\section{Dedicated to my}

Papa. Maa and my family

for belicuing in me, loving and supporting me

always in pursuit of fulfilling my dreams. 


\section{Acknowledgement}

First and foremost, I would like to thank the 'Almighty' for blessing me with the strength, perseverance and resilience to endure the hardships in the path to pursuing my dreams. I take this opportunity to thank and express my gratitude to each and every one who has been part of this amazing $\mathrm{PhD}$ journey and contributed to my successful completion of this thesis.

At the very outset, I would like to offer my sincere thanks to my primary supervisor, Dr Revti Raman, without whose support it would not have been possible for me to write this thesis. I would like to express my sincere gratitude to him for his valuable guidance, time, and collegiality. I cannot thank him enough for giving me the opportunity to be his mentee and for all the knowledge and wisdom that he has shared, which has helped me develop my research skills, and lay the foundation for my career as a researcher. I would also like to thank my secondary supervisors, Dr Yang Yu, and Dr Cheryl Rivers (former), for their valuable time and advice towards improving this thesis.

I would like to offer my gratitude to AProf Val Hooper, the Head of School of Marketing \& International Business (SMIB); the PhD Director Dr Micael-Lee Johnstone; the former PhD Director, Dr. James Richard; and the academic and professional staff of SMIB, who have always been there for us offering their constant support and assistance. I truly appreciate their efforts. Thanks for making this journey a pleasant one. I would also like to thank the respondents, the people associated with this research who offered their unconditional support and helped me conduct the study. I am thankful to the examiners who would put their valuable time and effort to reviewing this thesis.

I would like to especially thank all my $\mathrm{PhD}$ colleagues who have been part of this incredible journey. I am thankful to all of them for their love, motivation, companionship, and the knowledge that they shared, without which these four years would not have been the same. I would like to thank my family, friends, near and dear ones, for their enduring love and support. Last but not the least, I thank my parents, Tarun Phookan \& Bonti Phookan; and my family, Kaushik, Soumarika, Shivalika, and Alex. Without their love, support, and blessings this would not have been possible. 


\section{Abstract}

Cross border knowledge transfer is not only a major activity of multi-national enterprises (MNEs), but also the very reason for their existence. Most of the literature has investigated cross-border knowledge transfer at the firm level - with the headquarters or the subsidiaries as the actors. However, the action of knowledge transfer occurs between people within organizations but not between amorphous organizations. To account for the heterogeneous, independent individual behaviour, which may not always align with organizational objectives, I investigate interpersonal cross-border knowledge transfers (knowledge seeking and sharing) between subsidiaries in an MNE.

Based on the Social Identity Theory (SIT) insights, my proposed conceptual model hypothesised the impact of subsidiary power on interpersonal knowledge seeking and sharing being mediated by organisational identity of the individuals. It also includes two boundary conditions, intra-MNE competition and cultural intelligence, due to which knowledge transfer outcomes are likely to vary. The proposed conceptual model is tested using a questionnaire survey data from 333 employees from 40 R\&D subsidiaries of foreign MNEs in India. Before analysing the data with Conditional Process Analysis using the PROCESS macro within SPSS, a Confirmatory Factor Analysis was conducted with the help of AMOS.

The findings suggest that subsidiary power has a significant direct effect on knowledge sharing and an indirect effect on knowledge seeking. The results show that when it comes to seeking knowledge from another subsidiary, subsidiary power influences employees' seeking behaviour due to the organizational identification of employees. Whereas, although the decision to share knowledge is influenced by subsidiary power, it is due to factors other than identification. Further, cultural intelligence is found to moderate the indirect effect on knowledge seeking and intra-MNE competition moderates the indirect effect on knowledge sharing.

My study makes three key contributions. Firstly, I bring in SIT insights to the knowledge governance approach (KGA). I have argued and proposed identity based KGA mechanisms such as subsidiary power and intra-MNE competition which influence individual level knowledge transfer. Such mechanisms (although not governance mechanisms per se) can be 
used by the subsidiary to govern individual knowledge exchanges across the border. Secondly, I contribute by examining two boundary conditions for the subsidiary power and interpersonal knowledge transfer relationships. This explains under what conditions the effect of subsidiary power is strengthened or weakened. Finally, by conducting the study in the context of India, which is fast emerging as a R\&D hub for MNEs from different countries, the study provides insights to employees' knowledge exchange behaviour which is crucial for knowledge transfers within the MNEs and for their success. 


\section{Contents}

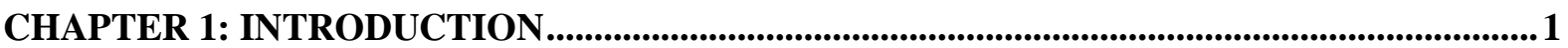

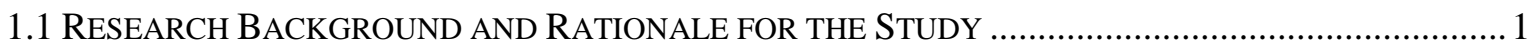

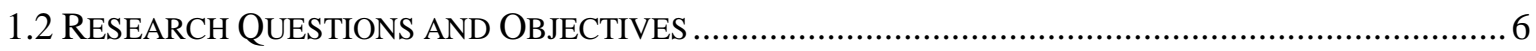

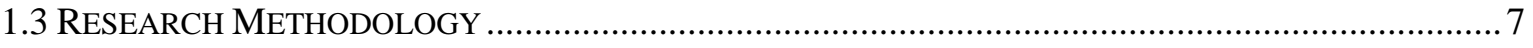

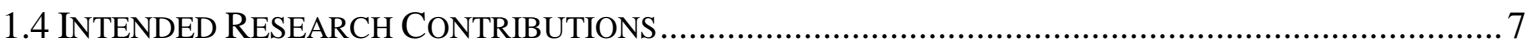

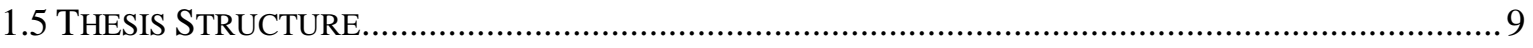

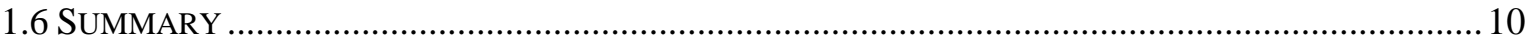

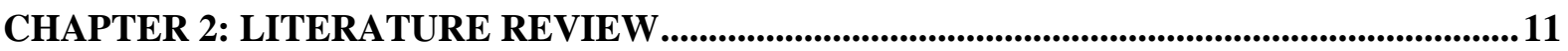

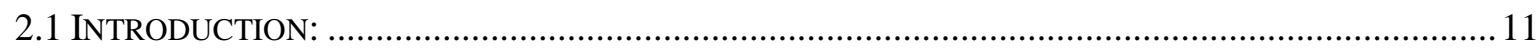

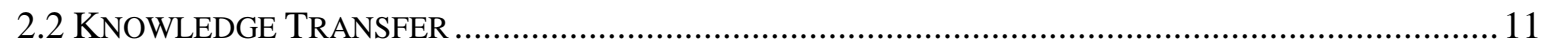

2.2.1 Organizational level knowledge transfer and organizational level antecedents: Quad 1 .... 14

2.2.2 Organizational level knowledge transfer and individual level antecedents: Quad 2...........16

2.2.3 Individual level knowledge transfer and individual level antecedents: Quad 3 ................. 18

2.2.4 Individual level knowledge transfer and organizational level antecedents: Quad 4 ...........21

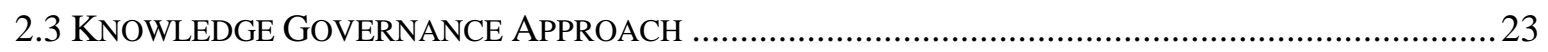

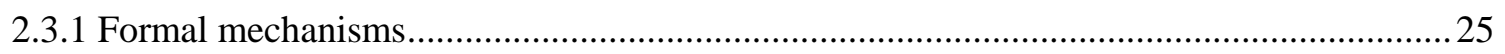

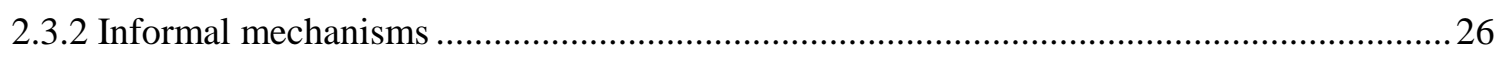

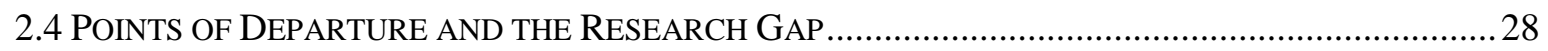

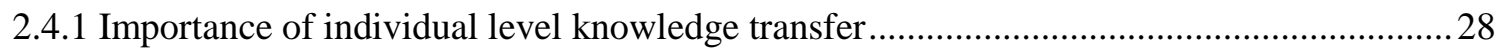

2.4.2 Individual level knowledge transfers in MNEs-filling the research gap .............................. 30

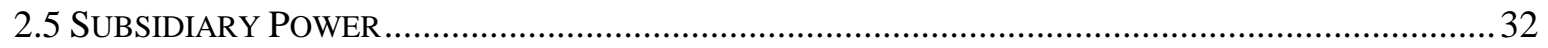

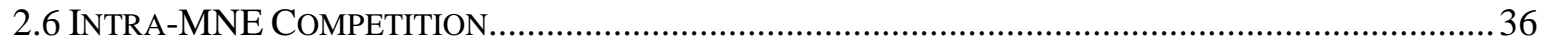

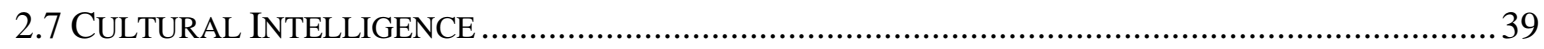

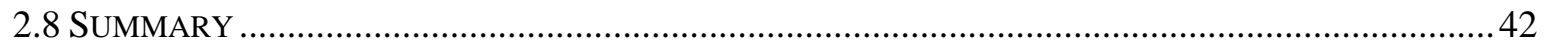

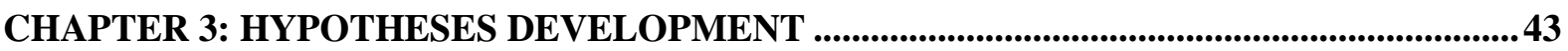

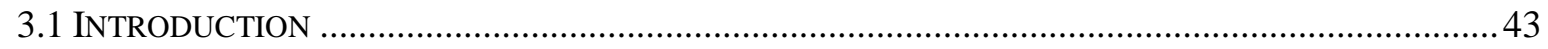

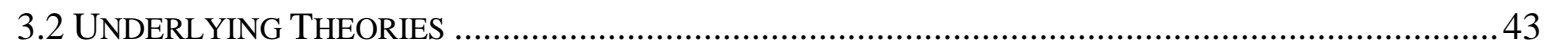

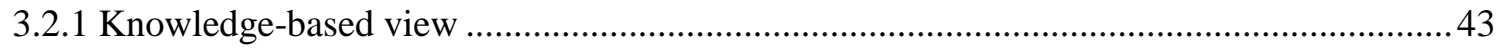

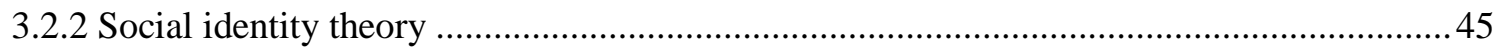

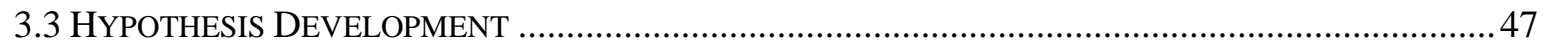

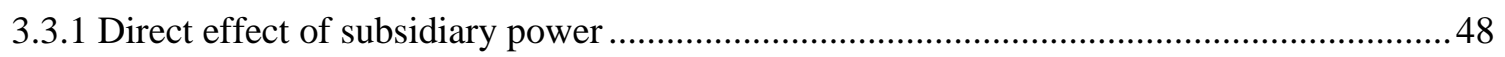

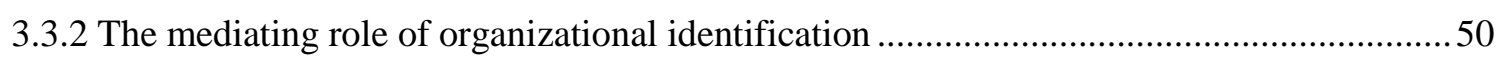

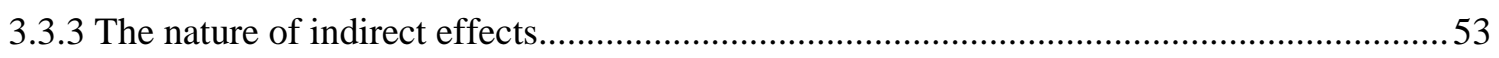




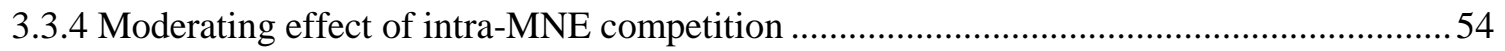

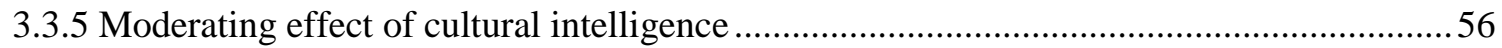

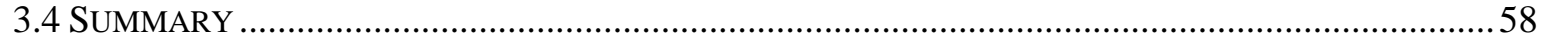

CHAPTER 4: RESEARCH DESIGN ....................................................................................................61

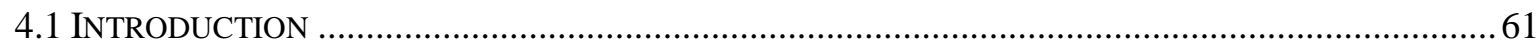

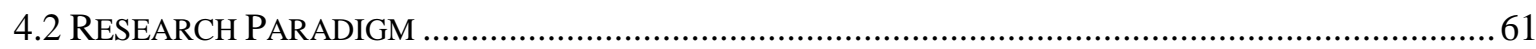

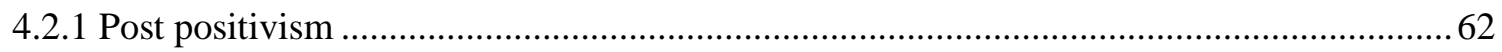

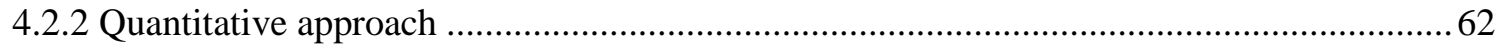

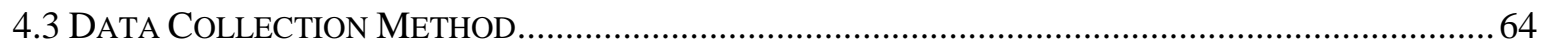

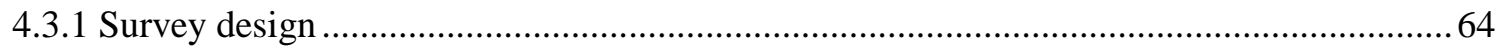

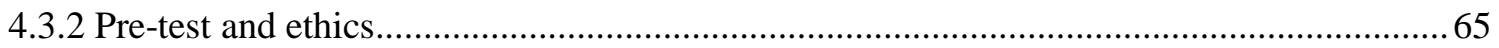

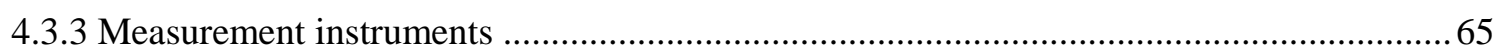

4.3.3.1 Dependent variable-interpersonal cross border knowledge transfer ...........................65

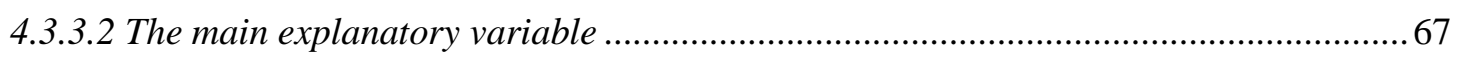

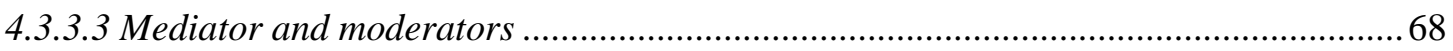

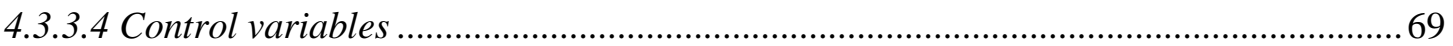

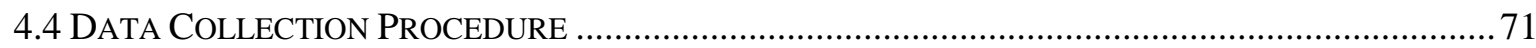

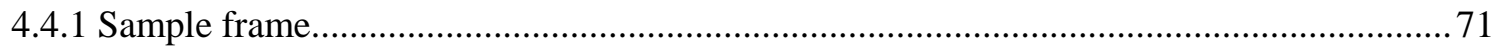

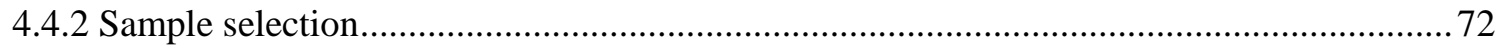

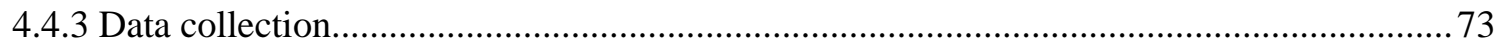

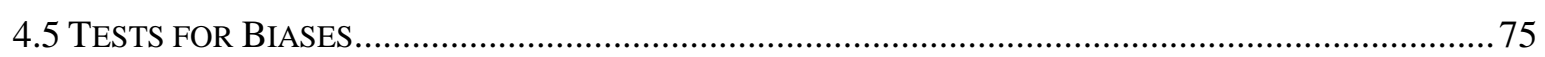

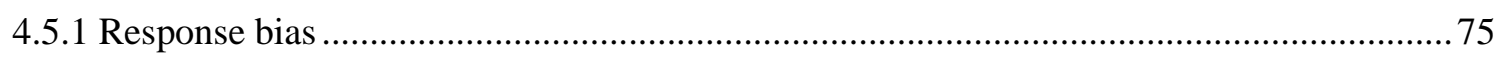

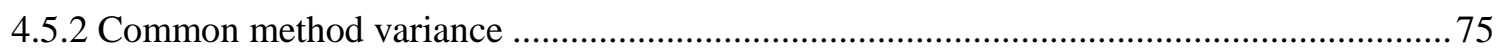

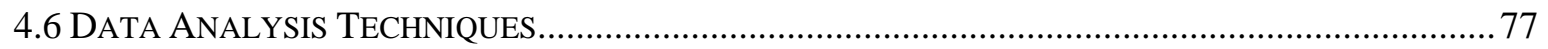

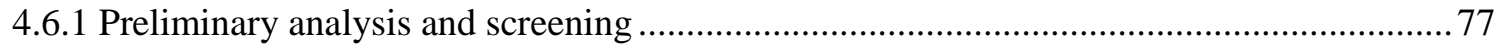

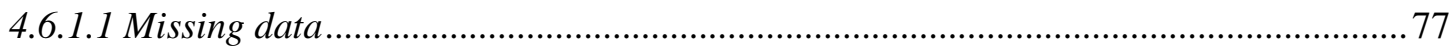

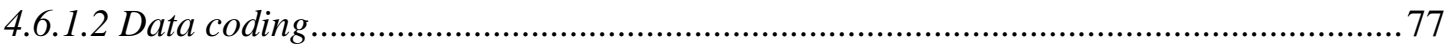

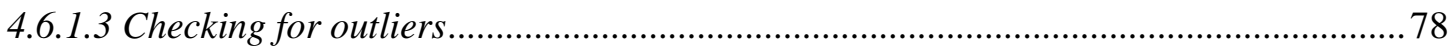

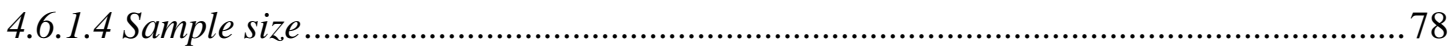

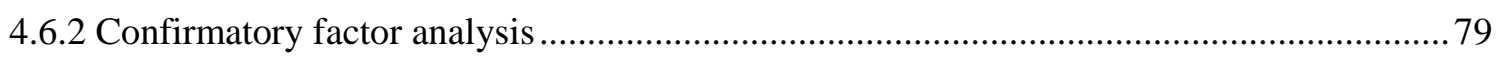

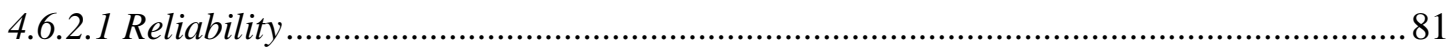

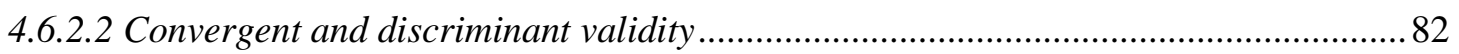

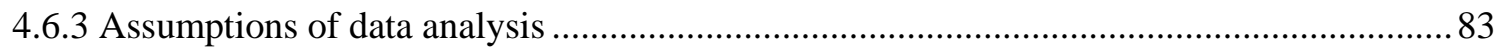

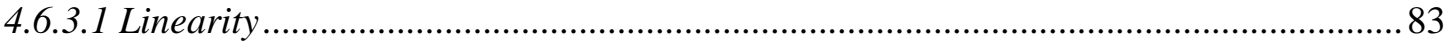

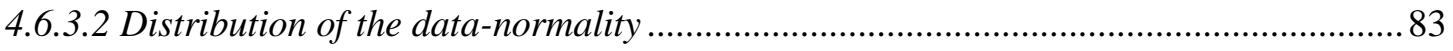

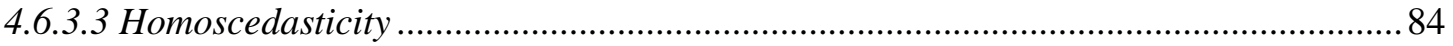




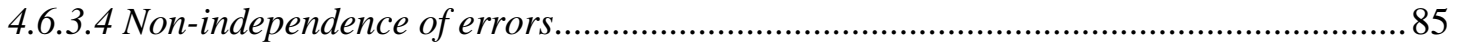

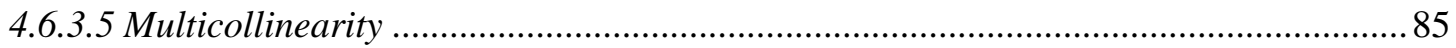

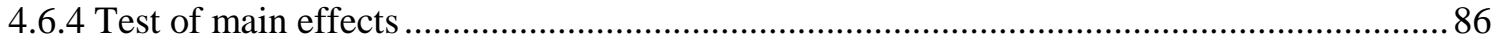

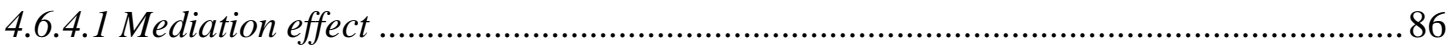

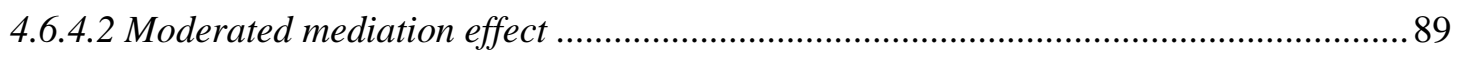

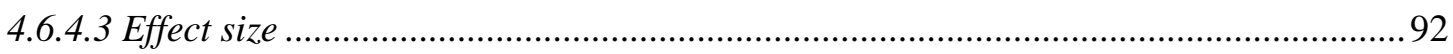

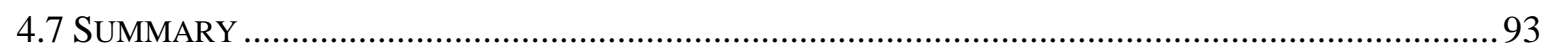

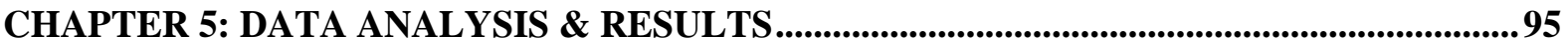

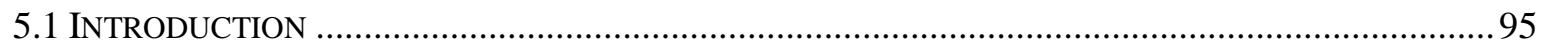

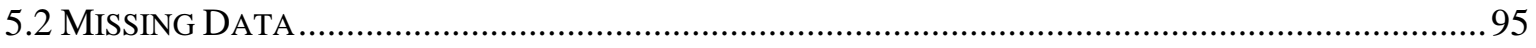

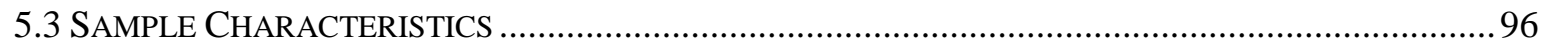

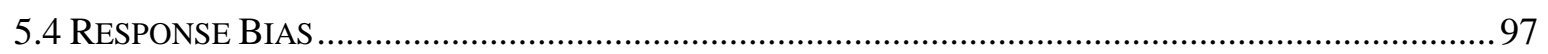

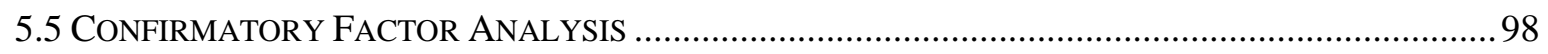

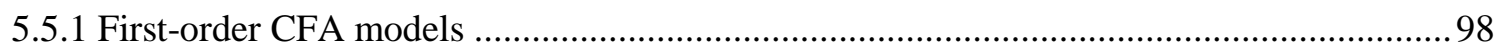

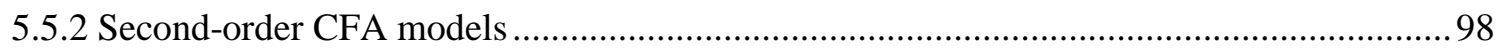

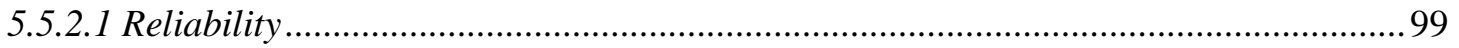

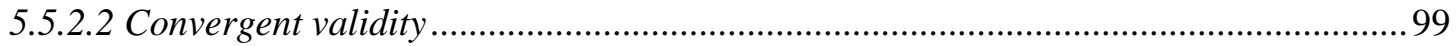

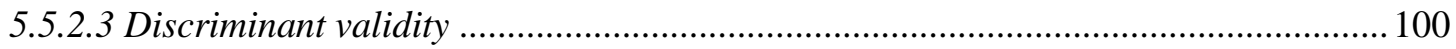

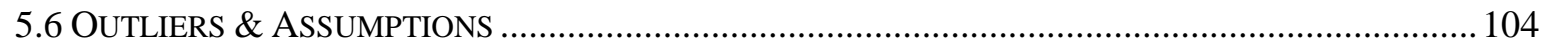

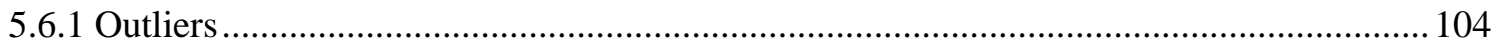

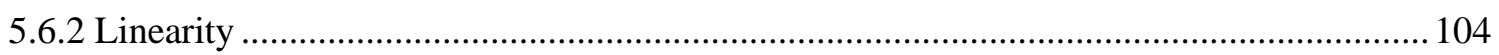

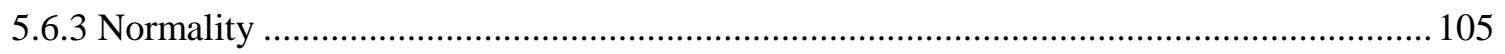

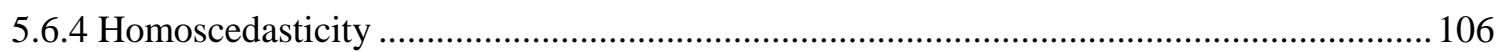

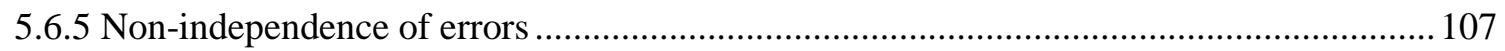

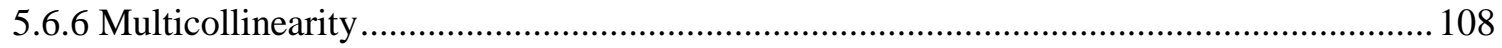

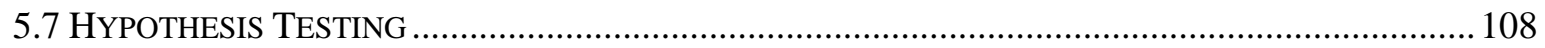

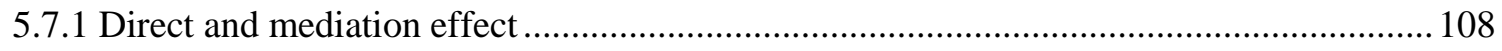

5.7.2 Moderation effect of competition and cultural intelligence ….......................................... 112

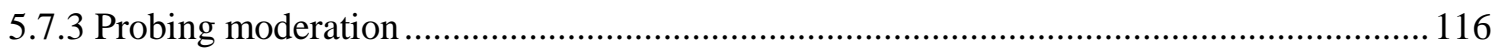

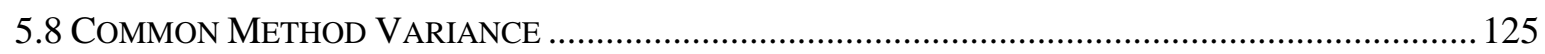

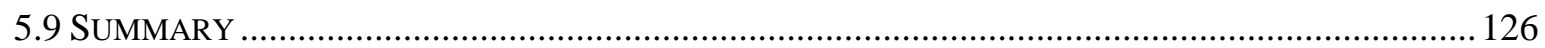

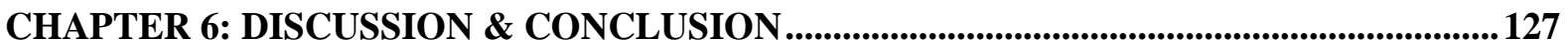

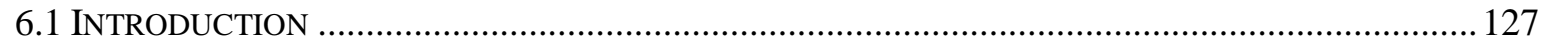

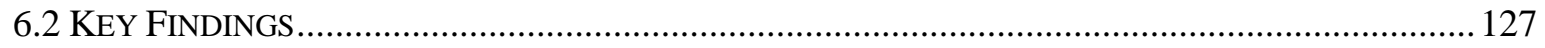

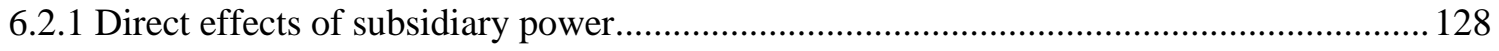


6.2.2 Indirect effect of subsidiary power.

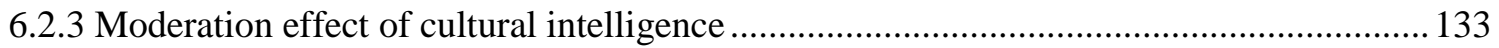

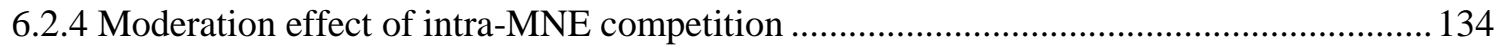

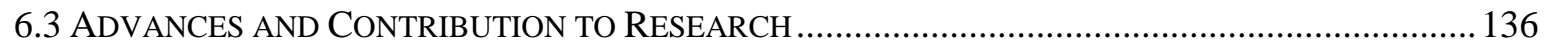

6.3.1 Cross-border individual level knowledge transfer in the MNE.......................................... 136

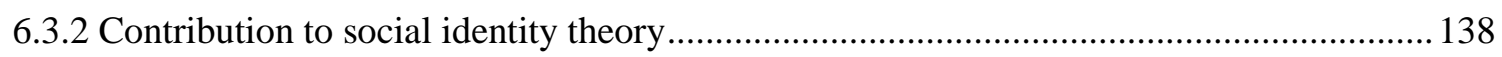

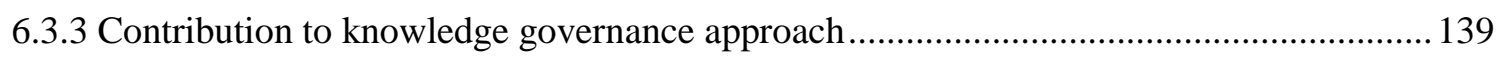

6.3.4 Contribution to understanding the context of study ........................................................... 141

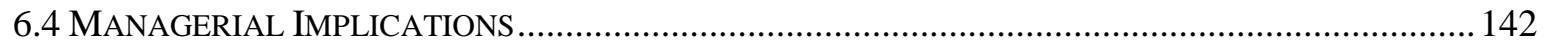

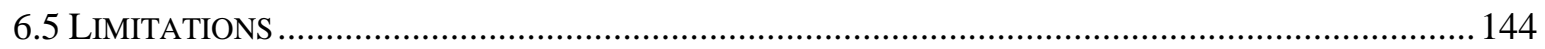

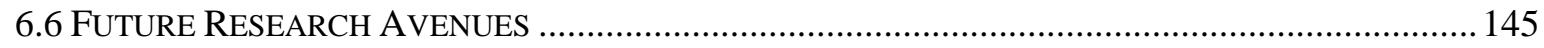

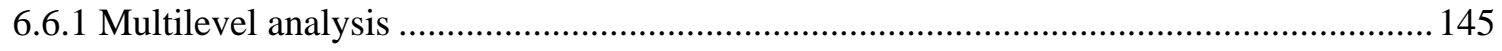

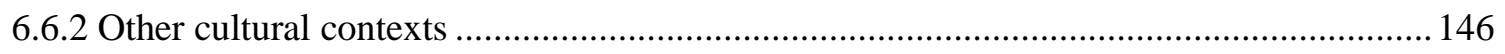

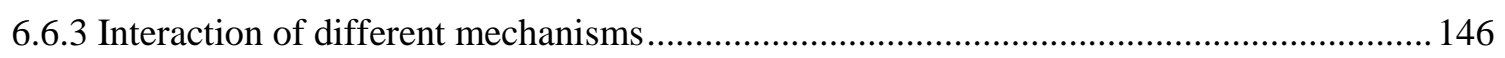

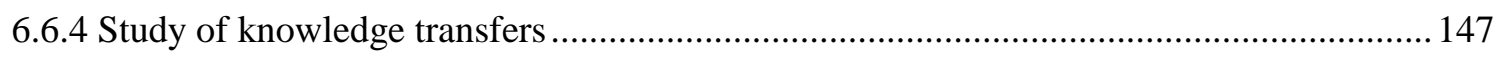

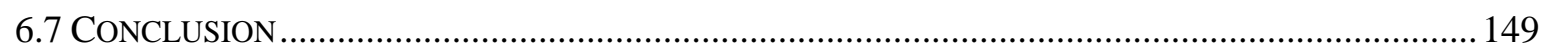

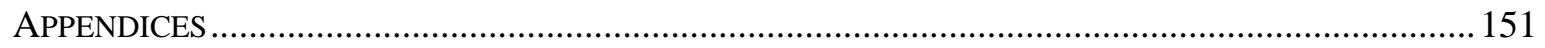

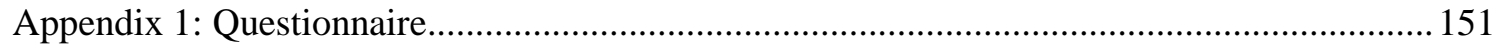

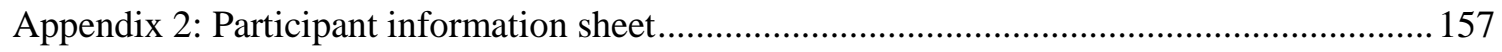

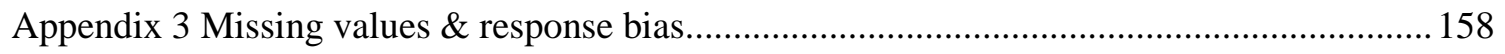

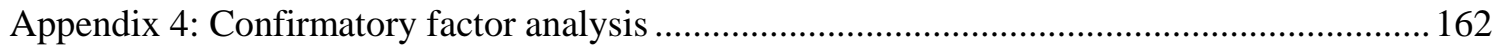

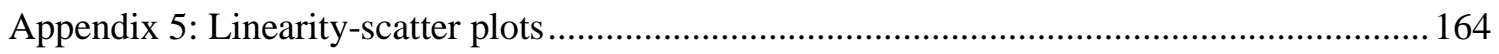

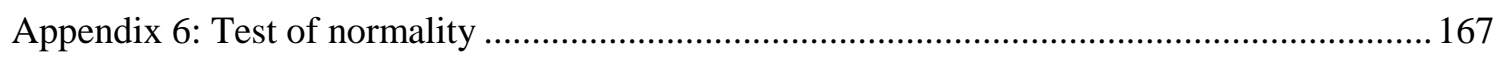

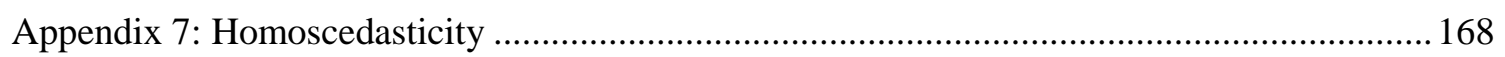

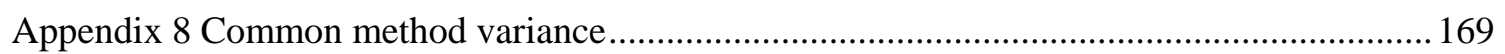

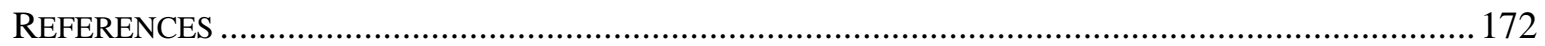




\section{List of Tables}

Table 4.1Empirical estimates of sample sizes needed for 0.8 power using percentile bootstrap ......... 79

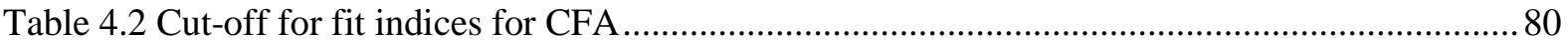

Table 5.1 Sample characteristics of respondents........................................ 96

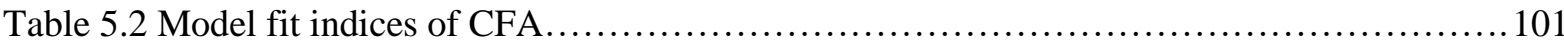

Table 5.3 Final measurement model item loadings, and reliability and validity test results 103

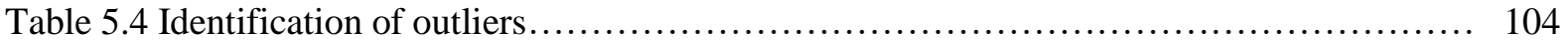

Table 5.5 Descriptive statistics- knowledge seeking................................... 106

Table 5.6 Descriptive statistics- knowledge sharing..................................... 106

Table 5.7 Intra-class correlations........................................................... 108

Table 5.8 Correlations output-Knowledge Seeking ........................................ 109

Table 5.9 Correlations output-Knowledge Sharing.................................... 109

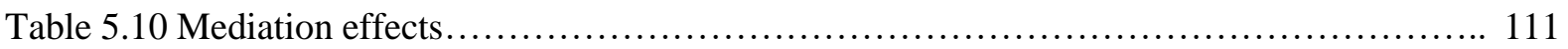

Table 5.11 Effect sizes................................................................. 111

Table 5.12 Indices of partial moderated mediation................................... 116

Table 5.13 Probing moderated mediation by cultural intelligence in the knowledge seeking

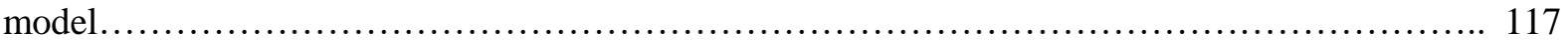

Table 5.14 Probing moderated mediation by intra-MNE competition in the knowledge sharing model........................................................................... 119

Table 5.15 Summary results- knowledge seeking ..................................... 122

Table 5.16 Summary results- knowledge sharing..................................... 123

Table 5.17 Summary of hypotheses tests findings..................................... 124

Appendix 3

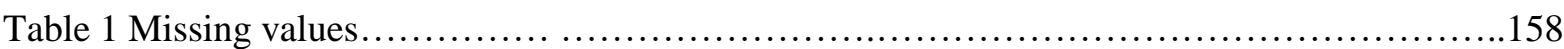

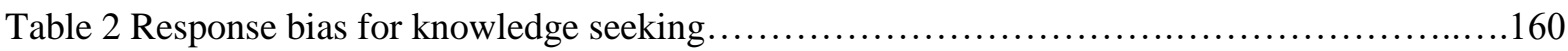

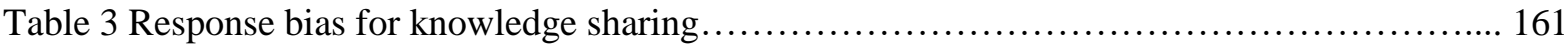

Appendix 6

Table 1 Kolmogorov-Smirnova test for knowledge seeking....................................167

Table 2 Kolmogorov-Smirnova test for knowledge sharing ...................................167

Appendix 7

Table 1 Breusch-Pagan and Koenker test for knowledge seeking.............................. 168

Table 2 Breusch-Pagan and Koenker test for knowledge sharing.................................168

Appendix 8

Table 1 Harman Single Factor Test for knowledge seeking ..................................... 169

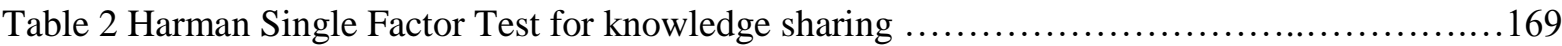

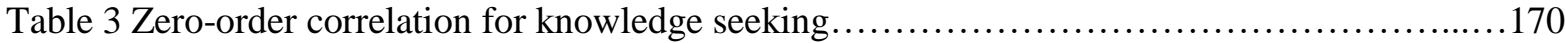

Table 4 Partial-correlation for knowledge seeking............................................ 170

Table 5 Zero-order correlation for knowledge sharing...................................... 171

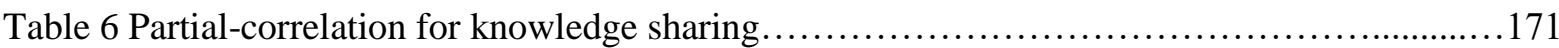




\section{List of Figures}

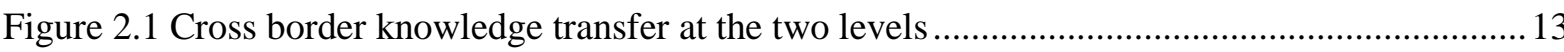

Figure 2.2 Interaction of knowledge transfer and its antecedents at two levels-individual and organizational

Figure 3.1 The conceptual model 48

Figure 4.1 Methodological fit 63

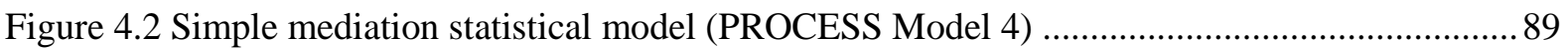

Figure 4.3 Moderated mediation statistical model (PROCESS Model 16).........................................92

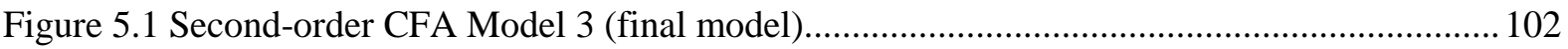

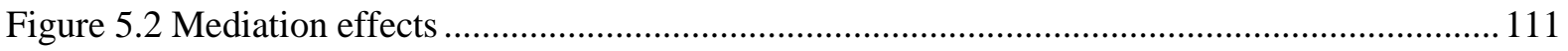

Figure 5.3 Moderation of OI on knowledge seeking and sharing by competition and cultural

intelligence

Figure 5.4 Moderated mediation effect

Figure 5.5 Interaction effect of CQ keeping competition constant at low, moderate and high levels 118

Figure 5.6 Moderation by CQ on the indirect effect

Figure 5.7 Interaction effect of competition keeping CQ constant at low, moderate and high levels 120

Figure 5.8 Moderation by competition on the indirect effect ........................................................ 120

Figure 6.1 Quasi-formal identity based mechanisms ..................................................................... 140

Figure 6.2 Countries from which knowledge is received and with which it is shared by the Indian subsidiary.....

Appendix 4

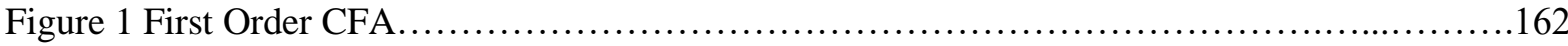

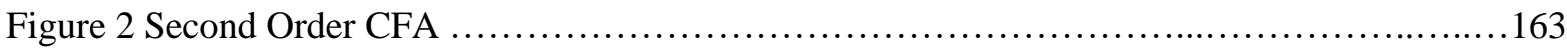

Appendix 5

Figure 5a The scatter plots of residuals of the combined independent variables on the dependent are

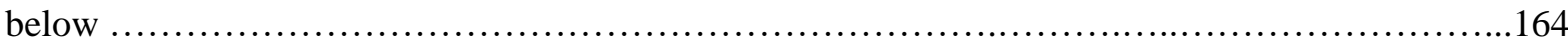

Figure $5 \mathrm{~b}$ The partial plots of each independent variable with the dependent variable................165

Appendix 7

Figure 1 Homoscedasticity Scatterplot for knowledge seeking ................................ 168

Figure 2 Homoscedasticity Scatterplot for knowledge sharing .................................168 


\section{List of abbreviations}

\begin{tabular}{|c|c|}
\hline MNE & Multinational Enterprise \\
\hline CBKT & Cross-border knowledge transfer \\
\hline SIT & Social Identity Theory \\
\hline KBV & Knowledge Based View \\
\hline OI & Organizational Identification \\
\hline CQ & Cultural Intelligence \\
\hline Comp & Intra-MNE competition \\
\hline KGA & Knowledge Governance Approach \\
\hline $\mathrm{R} \& \mathrm{D}$ & Research \& Development \\
\hline HQ & Headquarters \\
\hline CFA & Confirmatory Factor Analysis \\
\hline HRM & Human Resource Management \\
\hline SDT & Self-determination Theory \\
\hline WOS & Wholly owned subsidiary \\
\hline CMV & Common Method Variance \\
\hline SPSS & Statistical Package for the Social Sciences \\
\hline $\mathrm{CR}$ & Composite Reliability \\
\hline AVE & Average Variance Extracted \\
\hline MSV & Maximum Variance Extracted \\
\hline OLS & Ordinary Least Squares \\
\hline VIF & Variance Inflation Factor \\
\hline $\mathrm{T}$ & Tolerance \\
\hline SEM & Structural Equation Modeling \\
\hline TLI & Tucker-Lewis Index \\
\hline CFI & Comparative fit Index \\
\hline RMSEA & Root Mean Square Errors of Approximation \\
\hline IFI & Incremental Fit Index \\
\hline AIC & Akaike Information Criterion \\
\hline K-S & Kolmogorov-Smirnov \\
\hline HCSE & Heteroscedasticity-consistent Standard Error \\
\hline ICC & Intra-class Correlation \\
\hline SD & Standard Deviation \\
\hline
\end{tabular}




\section{Chapter 1: Introduction}

\subsection{Research Background and Rationale for the Study}

Knowledge transfer refers to the process by which knowledge is moved across the firm in order to leverage the firm's knowledge base (Argote, 1999; Patriotta, Castellano, \& Wright, 2013). The need to transfer knowledge and capabilities embedded in the geographically dispersed network nodes lies in the view of the MNE as a network organization (Ghoshal \& Bartlett, 1990; Ghoshal \& Nohria, 1989). Cross-border knowledge transfer is of crucial importance to multinational enterprises (MNEs) because effective internal transfer of knowledge has been linked to better coordination (Szulanski, 2000), innovation (Kotabe, Dunlap-Hinkler, Parente, \& Mishra, 2007; Phene \& Almeida, 2008), and to its competitive advantage (Argote \& Ingram, 2000; Grant, 1996; Kogut \& Zander, 2003). Indeed, Kogut and Zander (1993) attribute internalization of the firms and creation of the multinationals to their ability to transfer their idiosyncratic knowledge across national borders. Thus, intra-MNE cross-border knowledge transfer is not only a major activity of MNEs, but also the very reason for their existence.

Broadly, knowledge transfer happens at two levels in the organization: organizational level and individual level. Organizational level transfers take place between the headquarters (HQ) and subsidiary or between subsidiaries. Individual level knowledge transfers are interpersonal exchanges that take place between employees situated in different subsidiaries or in a HQ. Although the actors in an organizational level knowledge transfer are the HQ and subsidiaries, the action of knowledge transfer occurs between people within organizations and not between amorphous organizations (Argote \& Ingram, 2000). Most of the studies have examined intra-MNE knowledge transfer at the unit level at the cost of individual level analysis of the phenomenon (Dasí, Pedersen, Gooderham, Elter, \& Hildrum, 2017; Gupta \& Govindarajan, 2000; Michailova \& Mustaffa, 2012; Minbaeva, Pedersen, Björkman, Fey, \& Park, 2003; Schulz, 2003). This lacks complete understanding as the micro analysis view shows that knowledge transfer takes place at the individual level (Argote \& Ingram, 2000). 
Typically, organizational level knowledge flows are labelled as 'knowledge transfer' and individual level as 'knowledge flows' or 'exchanges' consisting of knowledge seeking and sharing (Minbaeva, Mäkelä, \& Rabbiosi, 2012). For the sake of simplicity, I use the terms knowledge 'transfer', 'flows' and 'exchanges' synonymously and interchangeably, which involves the two separate activities of 'seeking' and 'sharing' of knowledge. As proposed by the knowledge-based view, individuals are the locus of knowledge in the organization as knowledge creation, storage and efficient transfer depends upon individual specialization (Grant, 1996). The interpersonal knowledge exchange relationships are conduits of knowledge and information flow in the organization (Nebus, 2006). They are not only the channels of knowledge flows, but also the "prisms" that shape perception (Podolny, 2001) due to the exchange of opinions and insights between individuals. From the point of view of a focal individual, this involves two activities; seeking and sharing knowledge.

Interpersonal cross-border knowledge transfers involve both formal and informal exchanges. While formal transfers are under the directives of the organization, informal transfers are voluntary and outside organizational directives. The decision of an individual to seek knowledge from someone and share with someone is personal to the individual. Critical information gets transferred through these informal exchanges which facilitate knowledge assimilation and dissemination across the MNE (Bouty, 2000; Hippel, 1987; Kreiner \& Schultz, 1993). This substantiates the need to explore individual level cross-border knowledge transfers in MNEs.

However, individuals, as agents of interpersonal knowledge transfers are heterogeneous (Felin \& Hesterly, 2007). Knowledge exchange behaviour is also less likely to be homogeneous across different employees. It is independent to each individual and may not always align with the goals of the organization (Bjerregaard \& Klitmoller, 2016). Knowledge transfer is a multilevel concept as its antecedents at both organizational and individual levels impact the phenomenon. Apart from each individual's personal characteristics, factors such as the relationship between the exchange partners, analysis of costs and benefits, expectation of reciprocity, competition, as well as status and power may influence an individual's decision to seek or share knowledge with another individual (Caimo \& Lomi, 2015; Haas \& Cummings, 2015; Kachra \& White, 2008; Park, Chae, \& Choi, 2017; Wang \& Noe, 2010).

Antecedents that promote knowledge sharing across organizational units are different and distinct from knowledge sharing within the organization (Dasí et al., 2017). Knowledge exchange between individuals across subunits in an MNE is characterised by heterogeneous conditions and variability of the context. The effect of organizational separation in MNEs is 
"distinct from (and additional to) the various types of 'distance' that are found to be affecting knowledge sharing, as identified by the IB literature" (Dasí et al., 2017, p. 432). This calls for the need to identify those factors that emerge as a result of organizational separation of the business units in the MNE which may hinder the individual knowledge exchange behaviour across business units.

As subsidiaries within an MNE have different roles and the HQ-Subsidiary relationships vary accordingly, the MNE is far from being a "unitary organization"; rather, it can be conceptualized as an "inter-organizational network" where the units are embedded in a network of customers, suppliers or such other external actors (Ghoshal \& Bartlett, 1990). Based on the roles, responsibilities, and idiosyncratic characteristics, a subsidiary can gain more bargaining power within the MNE and exert considerable influence on MNE decisions (Ciabuschi, Dellestrand, \& Kappen, 2012; Mudambi \& Navarra, 2004). Therefore, power is important to a subsidiary as it can have great influence over various MNE processes which impact the subsidiary's development as well as its survival in the MNE (Balogun, Jarzabkowski, \& Vaara, 2011).

Owing to the fact that subsidiary power is important for a subsidiary and dynamics of power causes power to ebb and flow within the MNE, it is likely to influence each individual employee's knowledge exchange behaviour across different units. Knowledge held by the subsidiary is a source of its competitive advantage (Argote \& Ingram, 2000) and also a source of its power (Mudambi \& Navarra, 2004). By monitoring knowledge flows to and from the subsidiary, subsidiary members will try to protect and increase their knowledge and enhance their knowledge base to maintain or improve their current power status.

The idea of subsidiary power influencing individual knowledge exchange behaviour is underpinned by the Social Identity Theory (SIT) advocated by Tajfel (1978) and Turner (1975). Due to organizational separation and separate group identification at the subsidiary and the global organizational level, subsidiary employees distinguish between themselves and employees of other subsidiaries in the MNE (Gregersen \& Black, 1992). Power as a distinctive characteristic of the subsidiary, distinguishes it from other subsidiaries and confers the employees affiliated to it with enhanced organizational identification (OI). If an individual identifies strongly with a group, group norms affect his/her behaviour (Terry, Hogg, \& White, 1999). The group norms influence beliefs and behaviours that promote optimum maximization of inter-group differences and minimization of in-group differences (Terry \& Hogg, 1996). It is organizational identification through which subsidiary power is likely to influence individual knowledge exchange behaviour across subsidiaries. 
Given that MNEs are "complex organizational entities with intricate and multifaceted internal political processes" (Geppert, Becker-Ritterspach, \& Mudambi, 2016, p.2), typically there is ongoing competition and bargaining within the MNE. The MNE has been viewed as an internal market system, where subsidiaries participate in internal markets for goods and services, charters, competencies and practices (Cerrato, 2006). Subsidiaries within the MNE compete for parent's resources, mandates, system positions and customers (Becker-Ritterspach \& Dörrenbächer, 2011). Competition drives subsidiaries to take initiatives and to develop or acquire mandates (Birkinshaw, 1996), or engage in issue selling (Dörrenbächer \& Gammelgaard, 2016; Ling, Floyd, \& Baldridge, 2005), rent-seeking behaviour (Mudambi \& Navarra, 2004), or other profile building activities to attract headquarters' attention (Bouquet \& Birkinshaw, 2008).

Subsidiary employees are important actors in this intra-firm competition. Competition affects negotiations between subsidiary managers as they have the ability to mobilize resources based on their resource exchange relationships within and outside the MNE (BeckerRitterspach \& Dörrenbächer, 2011). Competition may lead them to use the resource exchange relationships in the interest of their own subsidiary to improve bargaining power (Gammelgaard, 2009). As found by Nguyen, Ngo, Bucic, and Phong (2018), cross-functional knowledge exchanges through informal relationships fail due to competition between departments. Additionally, competition between subunits is also a driver of members' identification with the subunit. In knowledge exchange relationships, employees try to protect their knowledge by sharing less or hide their knowledge gap, as knowledge is a source of power for a subsidiary (Mudambi \& Navarra, 2004) and would help it gain a competitive edge in intra-MNE competition. Therefore, this study proposes that intra-MNE competition is likely to influence the relationship of subsidiary power on interpersonal knowledge transfer through organizational identification.

Another factor that is likely to influence the effect of subsidiary power on interpersonal knowledge transfers is the cultural intelligence (CQ) of the employees. Culturally intelligent people are not only aware of and knowledgeable about another culture, but also have the ability to adapt their thinking and direct their behaviour to suit the cultural preferences of the other culturally different people (Thomas et al., 2008). Being sensitive to the cultural needs of a foreign culture makes one open-minded, flexible and capable of easily interacting with someone from a foreign culture (Imai \& Gelfand, 2010). This promotes cooperative behaviour with culturally different others. 
High CQ individuals work with a collaborative motive. They are less likely to engage in stereotyping and maintaining in-group out-group differences (Imai \& Gelfand, 2010) that arise from identification with their own subsidiary. They are able to overcome any prejudice that arises due to differences in culture. Greater respect for the values and customs of another culture and an ability to view from another's perspective helps develop shared understanding and shared identity with the possible outgroup (Kim \& Dyne, 2012). This will underscore the presence of superordinate identity, which is the MNE- the subsidiaries share.

Cultural intelligence plays an important role in cross-cultural interactions and knowledge transfers in MNEs (Vlajčić, Caputo, Marzi, \& Dabic', 2019). Involvement of individual actors makes individual cognitive skills important for interpersonal interactions. Right from the stage of initiation to successful transfer of knowledge, an individual's cultural capabilities play a crucial role. Cultural capabilities not only bring confidence to initiate a transfer process, but also help effectively conduct the transfer through better communication due to understanding of the other culture one is interacting with. Therefore, in the context of cross-unit knowledge transfers in MNEs, a higher CQ is likely to strengthen individual knowledge exchange behaviour.

This study examines knowledge transfers in Research and Development (R\&D) units of MNEs in India. Examining individual knowledge exchange across R\&D units of an MNE provides an appropriate context due to higher knowledge exchanges taking place between $R \& D$ units. Traditionally, R\&Ds were set up with an objective to transfer HQ or home country knowledge and technology. But presently, they act as a source of new knowledge for the whole organization due to their ability to capture spill-overs of external knowledge (Feinberg \& Gupta, 2004). MNEs continuously create, transfer and exploit knowledge dispersed across different R\&D subsidiaries around the world (Bartlett \& Ghoshal, 1989). Various studies have highlighted the existence of informal interpersonal knowledge exchange networks between researchers/scientists in R\&Ds which foster innovation in the organization (Allen, 1977; Bouty, 2000; Kachra \& white, 2008).

India is one of the fastest growing economies and is fast emerging as a major destination for MNEs for high-end R\&D projects (Deloitte, 2011; Gupta \& Gupta, 2014). According to a report from Zinnov, a Global Management Consulting firm, India hosted 1,165 R\&D centres at the end of 2015 established by 928 foreign MNEs, with an employment base of 323,000 scientists and engineers. These R\&D centres, which serve the Asia Pacific region and also the global market, are considered at par with those in the US, Europe or any other advanced countries (Motohashi, 2015). The MNEs having their R\&D units in India also have units across 
different countries, mainly in the US, UK, Europe, Japan, China, South Korea, and Israel. This makes knowledge transfer between R\&D units in India and units across these countries inevitable. $R \& D$ units provide a more appropriate environment for investigating knowledge transfers in multinationals (elaborated in section 4.4.1, Methodology chapter p. 71). An investigation of the knowledge exchange behaviour of Indian employees with their counterparts in other countries not only enhances our understanding of cross-border knowledge exchange within the MNE, but also provides insights on behaviours of employees set in an emerging economy.

\subsection{Research Questions and Objectives}

Drawing on the above research background, this study aims to address the following research questions:

- How does subsidiary power impact cross-border interpersonal knowledge transfer of the employees?

- How does intra-MNE competition between subsidiaries and cultural intelligence of employees influence the impact of subsidiary power on cross-border interpersonal knowledge transfer?

The proposed research questions are investigated by addressing the following research objectives:

- Examine how subsidiary power impacts interpersonal knowledge transfer. Interpersonal knowledge transfer implies two different directions of knowledge transfer; inflows and outflows to and from the subsidiary. These transfers are studied by examining two separate activities; knowledge seeking behaviour of an employee for inflows, and knowledge sharing behaviour of an employee (when someone else seeks) for knowledge outflows.

- Examine the indirect effect of subsidiary power on knowledge transfer through organizational identification (OI). That is, the mediating effect of OI on the relationship between subsidiary power and knowledge seeking and sharing.

- Examine the moderating effect of intra-MNE competition (comp) and cultural intelligence (CQ) on the relationship between OI and knowledge transfer.

- Examine the moderating effect of intra-MNE competition and cultural intelligence on the indirect effect of subsidiary power on knowledge transfer. 


\subsection{Research Methodology}

To investigate the above research questions and accomplish the objectives of this research, a quantitative research approach is used considering the maturity of the concepts and variables used in the study (Edmondson \& McManus, 2007). A model showing the linkage of concepts and variables used in the study and hypotheses for the relationships is proposed. To validate the conceptual model, a primary data collection technique is used. A questionnaire survey is used to collect data from individual respondents; researchers/scientists working in R\&D units of foreign MNEs in India. India, being one of the primary R\&D hubs of MNEs from the US, UK, and Europe, provides an appropriate context to examine cross-border knowledge exchange behaviour of individual employees in an MNE. This study collected 333 individual responses from employees working in 40 different foreign MNEs in India.

Hypotheses testing is conducted using a type of structural equation modelling, PROCESS Macro (Hayes, 2018a). Basically, it provides multiple regression analysis results with biascorrected bootstrap confidence intervals for the relationships. Before analysing the relationships, the data is cleaned, coded, and refined, and Confirmatory Factor Analysis is undertaken using AMOS in SPSS version 23 (Byrne, 2010). Validity and Reliability tests are conducted to validate the accuracy and consistency of the measurement of constructs. Assumptions of linear regression are also tested. Response bias is examined for responses collected using a paper-based survey against online surveys and for earlier and late responses. After conducting the hypotheses tests, a robustness test for common method variance is conducted using a marker variable technique (Lindell \& Whitney, 2001).

\subsection{Intended Research Contributions}

This research intends to make the following theoretical and empirical contributions to advance research on knowledge transfer in MNEs.

1. Presenting a comprehensive literature review by exploring studies on cross-border knowledge transfer at two different levels; organizational and individual, as well as their antecedents at both the organizational and individual levels, this review validates the need to study cross-border individual level knowledge transfer process in the MNE. By doing so, this research is able to identify and find evidence that cross-border individual level knowledge transfer, which is crucial for the MNE, has received limited attention. Therefore, by exploring cross-border individual knowledge transfers in the MNE, this research intends to contribute and advance our understanding and knowledge on the knowledge transfer discourse in MNEs. Moreover, unlike most other discourses 
which examine sharing of knowledge only, individual knowledge transfer is investigated by examining both the activities of knowledge seeking and sharing. This reflects a view that these are two distinct activities and therefore, need to be examined separately.

2. This study draws on the Social Identity Theory, from the social psychology discipline, and applies organizational identity insights to develop a theoretical framework to explain the link between subsidiary power and individual level knowledge transfer. In earlier studies, a relational perspective of organizational identity was used to examine knowledge transfers, where superordinate identity with the contact, the HQ, was used as a bonding agent to facilitate knowledge transfers. However, this study has presented another facet of organizational identification. Rather than helping knowledge transfers, OI may create barriers to the process or make knowledge sticky. This aspect of organizational identification of employees is unique to an MNE, where subsidiary units are separated by differences in geography and contexts. Through this, the study contributes to SIT by advancing our knowledge about OI in organizations.

3. This study contributes to the Knowledge Governance Approach (Foss, 2007). Being able to govern knowledge exchange behaviour of employees is an important focus of knowledge management and human resource management in the organization. The KGA contributes to this discourse by bringing in organizational factors which help in governing individual knowledge transfers. KGA makes a distinction between formal and informal governance mechanisms. This research proposes an additional set of organizational level identity-based factors to KGA, which although not governance mechanisms per se, can nevertheless be used to govern knowledge exchanges in the MNE. This study calls these quasi-formal governance mechanisms.

4. Additionally, two boundary conditions are identified; intra-MNE competition and cultural intelligence, for the relationships between subsidiary power and knowledge transfer. These boundaries explain that the impact of subsidiary power on knowledge transfer varies for two different conditions, the extent of intra-MNE competition and cultural intelligence of employees.

5. By understanding the interplay of organizational identification, subsidiary power, intraMNE competition and cultural intelligence in predicting employee knowledge exchange behaviour, recommendations are put forward to strategists and HR managers to facilitate cross-border knowledge transfers within the MNE by applying new identity-based governance mechanisms. Through manipulation of employee 
perceptions or managing employee identification with the subsidiary/HQ, managers will be able to govern knowledge exchanges across the border.

6. Lastly, as the context of the study is India, it gives us an opportunity to gain insights to individual behaviour in the unique multicultural context of India. As more and more MNEs are establishing their subsidiaries including R\&D centres in India, the findings are likely to be useful for MNEs to find ways to enhance interpersonal and organizational knowledge transfers to and from subsidiaries located in India or other emerging economies having a similar context.

\subsection{Thesis Structure}

The thesis comprises six chapters which are outlined below.

Chapter 1 introduces the topic of research and discusses the background, problem and rationale for the study. Additionally, the research questions, objectives, methodology adopted and intended contributions of the study are discussed.

Chapter 2 critically reviews literature on knowledge transfer in the MNEs. A comprehensive literature review is undertaken using a $2 \times 2$ matrix structure, exploring knowledge transfer at the organizational and individual levels and their antecedents at both the levels. From this review, patterns and gaps have been identified and the study is proposed. Also, the other constructs, subsidiary power, intra-MNE competition, and cultural intelligence used in the study are discussed.

Chapter 3 begins with a discussion of the theoretical underpinnings of the research-the Knowledge Based View and the Social Identity Theory. A conceptual model and a theoretical framework linking subsidiary power and knowledge transfer is presented. Later, hypotheses are developed for the proposed relationships between the constructs-subsidiary power, organizational identification, knowledge transfer, intra-MNE competition and cultural intelligence.

Chapter 4 consists of discussion on research methodology, research design, construct measurement, sampling method, data collection procedure, and data analysis techniques used for the study.

Chapter 5 presents the refinement of the measures using CFA. Prior to analysis, assumptions of regression analysis are tested, and the descriptive statistics are presented. This chapter explains the data analysis and the hypotheses testing using conditional process analysis with Process macro. 
Chapter 6 provides a thorough discussion of the findings in relation to the research questions. Further discussion covers the theoretical contributions, managerial implications, and limitations of the study. This chapter concludes by putting forward recommendations for future research.

\subsection{Summary}

In this chapter I have presented an overview of my research study. At the outset, I present a background of the study and uphold the rationale for researching the particular topic of study. Next, I propose the research questions of interest and the methodology used to conduct the study. Later, I put forward the intended research contributions of my study, and the thesis structure. In the next chapter I will present an elaborate review of the related literature. 


\section{Chapter 2: Literature Review}

\subsection{Introduction:}

This chapter reviews literature on cross-border knowledge transfer in the MNE. The chapter begins with a review of the literature on knowledge transfers using a $2 \times 2$ matrix where literature at both the organizational and individual levels is reviewed. Then, I have zoomed in on one of the four quadrants of the $2 \times 2$ matrix to elaborate the knowledge governance approach, which is of interest to this study. Based on this review, a research gap is identified and three means (subsidiary power, intra-MNE competition, cultural intelligence) are proposed to explore the gap. This is followed by literature review on these three constructs. Then, the chapter summary closes this chapter.

\subsection{Knowledge Transfer}

Davenport and Prusak (1998, p.5) define knowledge as "a fluid mix of framed experience, values, contextual information, and expert insight that provides a framework for evaluating and incorporating new experiences and information". They further argue that it resides in individuals; the knowers. Organizations embed knowledge in documents, routines, processes, practices, and norms. Knowledge has been categorized into product knowledge, process knowledge, and management knowledge (Grosse, 1996). Hansen (2002), Kogut and Zander (1992), and Minbaeva et al. (2012), categorized knowledge as information and know-how. Information refers to the knowledge which can be transmitted while keeping its integrity intact. Examples include facts, data, or documents which can be easily transferred without loss of value. Information also denotes- "know-what" something means. Whereas, "know-how" denotes knowing how to do something. "It is the accumulated practical skill or expertise that allows one to do something smoothly and efficiently" (Hippel, 1988). While "know-what" 
captures explicit knowledge which can be codified or documented; "know-how' is tacit or procedural knowledge which is difficult to code and mostly embedded in practices.

Following Hansen (2002), Kogut and Zander (1992), and Minbaeva et al. (2012), knowledge, as used in this study, means any 'information/know-what' and 'know-how'. 'Know-how' would capture the tacit knowledge, the uncodified undocumented knowledge, such as, the components of advice, problem solving, and brain storming. This knowledge is non-proprietary, not protected by patent and there is no formal embargo on its transfer (Kachra \& White, 2008). Knowledge that is highly tacit and cannot be exchanged cross-border through virtual media of communication is outside the purview of this study.

Knowledge transfer refers to the process by which knowledge is moved across the firm in order to leverage on the firm's knowledge base. It has been defined as the mechanism through which experiences of an individual or a unit affects other individuals or units in the organization (Argote, 1999; Patriotta, Castellano, \& Wright, 2013). Szulanski (1996, 2000) defines it as a dyadic process of knowledge exchange between a sender and a receiver, where the disposition and ability of the source and the recipient, strength of their tie and characteristics of the knowledge decides the effectiveness of the transfer (Minbaeva, 2007; Szulanski, 2003).

Knowledge transfer in a multinational organization is multi-dimensional and multidirectional (Minbaeva, 2007). It happens in four directions- 1) From HQ to Subsidiary (Vertical transfer); 2) From subsidiary to the HQ (Reverse transfer); 3) From a focal subsidiary to a peer subsidiary, and 4)From a peer subsidiary to a focal subsidiary (the latter two called lateral transfers) (Gupta \& Govindarajan, 2000). Intra-MNE knowledge transfer occurs at two levels: organizational level and individual level. At the organizational level knowledge flows from HQ to subsidiary/s, subsidiary/s to HQ and between subsidiaries across different countries. At the individual level, knowledge transfer can occur between individual employees either in units/subsidiaries or in HQ located in different countries. Likewise, the antecedents or the factors that may influence the processes are also at the two levels.

The review of intra-MNE cross border knowledge transfer is presented with a two-bytwo matrix representing four categories of different combinations of knowledge transfers and antecedents, at two different levels; organizational and individual (Figure 2.1). The figure categorizes the related literature into four quadrants. Quadrants 1 and 2 include the impact of organizational level and individual level factors respectively on organizational level knowledge transfer. Quadrants 3 and 4 present their impact on individual level knowledge transfer. Additionally, the base line includes literature on characteristics of knowledge which impact transfers at both the levels. 
Figure 2.1 Cross border knowledge transfer at the two levels

\begin{tabular}{|c|c|c|}
\hline \multirow{2}{*}{ Explanatory variables } & \multicolumn{2}{|c|}{ Outcome Variable: Knowledge Transfer } \\
\hline & Organisational Level & Individual Level \\
\hline Organisational Level & $\begin{array}{l}\text { Quad 1: Single level - rich Literature } \\
\text { Ability, Willingness of organization, absorptive } \\
\text { capacity, disseminating capacity, close } \\
\text { relationships, trust, subsidiary power and } \\
\text { autonomy, performance evaluation criteria, HRM } \\
\text { practices, knowledge management tools, } \\
\text { contextual similarity and differences. }\end{array}$ & $\begin{array}{l}\text { Quad 4: Multi-level-emerging literature } \\
\text { Knowledge governance approach, } \\
\text { organisational culture and values, HRM } \\
\text { Practices, social governance mechanisms, } \\
\text { commitment based mechanisms, national } \\
\text { Culture, language, functions. }\end{array}$ \\
\hline Individual Level & $\begin{array}{l}\text { Quad 2: Multi-level- emerging literature } \\
\text { Individual knowledge networks, informational } \\
\text { boundary spanners, managerial sense making, } \\
\text { micro foundations approach. }\end{array}$ & $\begin{array}{l}\text { Quad 3: Single level-rich Literature } \\
\text { Personality characteristics, attitude and } \\
\text { motivation, personal relations and networks, } \\
\text { demographic characteristics, individuals' } \\
\text { power, status, boundary spanning. }\end{array}$ \\
\hline Base Line & \multicolumn{2}{|l|}{ Characteristics of the knowledge transferred } \\
\hline
\end{tabular}

Source: Author's compilation based on review of the representative literature

Besides the exogenous factors, characteristics of the knowledge involved in the transfer affects the transfer process at both the levels (baseline, Figure 2.1). Knowledge includes both tacit and explicit (Holtbrügge \& Berg, 2004; Minbaeva, 2007; Pedersen, Petersen, \& Sharma, 2003; Polyani, 1966), complex and simple (Kogut \& Zander, 1993; Minbaeva, 2007), or declarative \& procedural (Noorderhaven \& Harzing, 2009) components. The degrees of complexity, teachability, and tacitness of knowledge affect the transfer process. The higher the degree, the harder and slower the transfer. As 'we know more than we can tell' (Polanyi, 1966, p.4) tacit knowledge is hard to be articulated, codified, stored and transferred (Li \& Gao, 2003). Tacit knowledge is difficult to transfer through virtual communication media, and requires rich communication channels such as colocation of the actors (Klitmøller \& Lauring, 2013; Pedersen et al., 2003).

However, as posited by Nonaka and Takeuchi (1995), tacit knowledge can be converted to explicit knowledge by the process of socialization, externalization, combination, and internalization, which they presented through the spiral SECI knowledge conversion model. They identified four different patterns of interaction between tacit and explicit knowledge and suggested that knowledge can be mobilized through the dynamic interaction between these four modes. These conversions involve processes such as shared experience, conversation, coordination, documentation, and learning metaphor. According to them, SECI originates at the level of the individual and transcends to the collective group level, organizational level and eventually to the inter-organizational level. 
Explicit knowledge is highly codified, easy to transmit, whereas tacit knowledge is more abstract, harder to articulate and transfer. Crespo, Griffith, and Lages (2014) found that explicit knowledge is positively related to knowledge outflows from the subsidiary to the HQ. While transfer of tacit knowledge primarily requires rich communication media, explicit knowledge can be transferred through written media (Pedersen et al., 2003). The more easily knowledge can be communicated due to easy articulability, codifiability and teachability, the faster is the speed of knowledge transfer in the organization (Zander \& Kogut, 1995).

\subsubsection{Organizational level knowledge transfer and organizational level antecedents:}

\section{Quad 1}

Intra-MNE knowledge transfer has been extensively studied in International Business and Management literatures. Such knowledge transfer takes place between headquarters and the subsidiaries, or between the subsidiaries. Traditional studies focused on knowledge transfer from headquarters to the subsidiaries, where the objective of the MNE has been international exploitation of its competitive advantage in the host countries (Gupta \& Govindarajan, 1991; Minbaeva et al., 2003; Noorderhaven \& Harzing, 2009; Schulz, 2003; Tseng, 2015). Knowledge transfer has also been studied between subsidiaries (Crespo, Griffith, \& Lages, 2014; Tsai \& Ghoshal, 1998; Zhao \& Luo, 2005). More recently, reverse knowledge transfers from the subsidiaries to the headquarters have been explored, where subsidiaries from emerging economies in particular, diffuse the knowledge tapped from local sources to other parts of the MNE (Ambos, Ambos, \& Schlegelmilch, 2006; Foss \& Pedersen, 2002; Hakanson \& Nobel, 2001; Mudambi, Pedersen, \& Andersson, 2014). It is noteworthy that in a majority of these studies, the designated actors are the headquarters or the subsidiaries. A plethora of studies have researched the antecedents of intra-MNE knowledge transfer at the organizational level.

Characteristics of the actors participating in the transfer influence effectiveness and efficiency of the process. Two important characteristics are the ability and willingness of the headquarters or the subsidiary to send or receive knowledge (Minbaeva, 2007; Noorderhaven \& Harzing, 2009; Tseng, 2015; Wang, Tong, \& Koh, 2004). The ability of a sender is determined by the knowledge base and skills of the employees involved in the transfer (Grant, 1996); and willingness is contingent on the benefits the sender will obtain from the transfer. A receiver's absorptive capacity (Cohen \& Levinthal, 1990), which involves two elements; prior knowledge and intensity of effort, is also a major determinant of the effectiveness and efficiency of the knowledge transfer process (Gupta \& Govindarajan, 2000; Minbaeva, 2007; 
Szulanski, 1996). The higher the ability and motivation of the employees in an organization to recognise, assimilate and apply (Cohen \& Levinthal, 1990, p. 128) external knowledge, the more knowledge will be transferred to the organization.

Not surprisingly, the nature of the relationship between the sender and receiver is also an important antecedent of knowledge transfer. Close relationships between the sender and receiver units due to frequent communication (Crespo et al., 2014), previous cooperation (Ciabuschi et al., 2012; Inkpen \& Tsang, 2005; Tsai \& Ghoshal, 1998; Uzzi, 1997; Zhao \& Luo, 2005), integrated work processes (Noorderhaven \& Harzing, 2009), and interdependent work flows (Bartlett \& Ghoshal, 1989) ease the process of international knowledge transfer by reducing motivational and cognitive problems (Szulanski, 1996). A close relationship which is conditioned by the extent and nature of trust in the relationship between the exchange partners influences knowledge transfers between them (Szulanski, 1996; Argote, McEvily \& Reagans, 2003; Welch \& Welch, 2008). Other relational factors such as identification with the source (HQ) or competition with peer subunits also impact knowledge transfer and implementation in the subsidiaries (Argote \& Kane, 2009; Hansen, Mors, \& Lovas, 2005; Kostova \& Roth, 2002; Tsai, 2002).

Organizations also use formal mechanisms such as giving more autonomy to the subsidiary (Ciabuschi, Martín, \& Ståhl, 2010), setting subsidiary performance evaluation criteria (Bjorkman, Barner-Rasmussen, \& Li, 2004), knowledge management tools (Mahnke, Pedersen, \& Venzin, 2005) or HRM practices (Minbaeva et al., 2003), with an aim to facilitate knowledge transfers between the units. Delegating decision-making rights to the subsidiary increases its perception of freedom and motivation to transfer more knowledge (Ciabuschi et al., 2010; Foss \& Pedersen, 2002). Formal organizational mechanisms can be used by the HQ as control mechanisms to impact ability and motivation of employees to transfer knowledge, leading to transfers between organizations. By deploying certain specific HRM practices a recipient unit's absorptive capacity can be developed which fosters more knowledge inflows (Zhou, Fey, \& Yildiz, 2018).

Homophily or similarity between the organizational contexts provides a common ground for understanding and exchange of knowledge which increases interaction between the parties resulting in more knowledge transfers. Context encompasses knowledge, values, assumptions and beliefs held by the exchange partners. Cultural similarity or low cultural difference (Schlegelmilch \& Chini, 2003), language similarity (Ambos \& Ambos, 2009; Reiche, Harzing, \& Pudelko, 2015), and organizational cultural difference (Cui, Griffith, Cavusgil, \& Dabic, 2006) positively influence knowledge transfers between units. Knowledge flows from units 
located in an advanced developed country to a unit located in a less developed country is greater, as the knowledge held by the developed country unit is perceived as more valuable than that of the other (Frenkel, 2008; Gupta \& Govindarajan, 2000).

Studies on contextual perceptions and knowledge transfers between subsidiaries imply competitive tension and the existence of a relationship of power and politics between the units (HQ-Subsidiary/subsidiary-subsidiary). A relationship based on power due to resource dependency can aid knowledge flows between the units. Wong, Ho, and Lee (2008) found that knowledge flows will be stronger to a high power unit from a low-power due to the low power unit complying with the demands of the high-power unit for knowledge. But this finding is conditional upon the high-power subsidiary seeking knowledge from the low-power agent. More recently Whittle, Mueller, Gilchrist, and Lenney (2016) contended that due to a relationship of power and politics between the HQ and the subsidiary, knowledge transfer between them is affected. Due to sensemaking of HQ's power over the subsidiary, subsidiary managers may hide or not share important information or knowledge of the local market with the HQ, which affects flows of subsidiary knowledge to the HQ. In view of the tension that may exist between the units, units are susceptible to trust issues or issues that feed a unit's perception of the other, which can make the knowledge sticky or create barriers to knowledge transfers.

\subsubsection{Organizational level knowledge transfer and individual level antecedents: Quad 2}

The knowledge-based view (Grant, 1996) suggests that the locus of knowledge is in the individual and the primary role of the firm is coordination and integration of this knowledge embedded in the individuals dispersed across different subsidiaries. People are identified as important conduits of organisational level knowledge transfer. Argote and Ingram (2000) suggest that as individuals are important reservoirs of knowledge, knowledge transfer between units takes place through interactions between individuals.

However, most of the studies have examined intra-MNE knowledge transfer at the unit level (Minbaeva et al., 2003). This lacks complete understanding as the micro analysis view argues that knowledge exchanges at the individual level are important for knowledge flows/transfers within the MNE (Argote \& Ingram, 2000; Felin \& Hesterly, 2007; Kostova, 1999). Examining the impact of individual factors on organizational knowledge transfer is in

line with the micro-foundations perspective (Felin, Foss, \& Ployhart, 2015). This field is informed by studies on individual level antecedents of organizational cross-border knowledge transfer. 
Studies on individual knowledge networks form an integral part of this classification. For transfer of inter-unit knowledge, Zhao and Anand (2013) proposed network structures between individuals located in different units which they called boundary spanner and collective bridge. According to them, knowledge with different degrees of complexity requires different types of network structures for transfer. Individuals participating in these networks exchange knowledge through interactions and aid transfers between units. Bjorkman et al. (2004) also found that corporate socialization mechanisms facilitate interpersonal networks between members of different units which increase interpersonal ties between units and lead to interunit knowledge transfer (Hansen, 1999; Tsai, 2001).

Another study on organizational knowledge transfer which is grounded on individual level antecedents is by Whittle et al. (2016). They found that individual actions of managers in subsidiaries may influence knowledge transfers at the organizational level. HQ and subsidiaries have their unique capacities and capabilities due to differences in the resources and knowledge possessed by them. Based on these resources and capabilities, managers in subsidiaries make sense of the power of their subsidiary vis-a-vis the power of the HQ over them. This sensemaking guides their reasoning and decision-making and leads them to censor important information from the HQ resulting in lesser flow of subsidiary knowledge to the HQ.

Additionally, although the concept of "Absorptive Capacity" (Cohen \& Levinthal, 1990), is a firm level concept, it resides with organization's employees as it is the interaction between employees' ability and motivation to acquire information (Minbaeva et al., 2003). Absorptive capacity of a unit positively influences inflow of knowledge to it. Organizations can use various governance mechanisms like HRM practices to increase the absorptive capacity of its employees resulting in increased knowledge transfers to the unit.

Furthermore, sometimes organizational level knowledge transfers occur due to movement of individuals between units. International assignees - expatriates, or repatriates, and global managers can act as informational boundary spanners and carriers of important organizational knowledge between units leading to intra-MNE knowledge transfers (Bjorkman et al., 2004; Chang, Gong, \& Peng, 2012; Furuya, Stevens, Bird, Oddou, \& Mendenhall., 2009; Lazarova \& Tarique, 2005; Patriotta et al., 2013; Tsang, 1999; Wang, Tong, Chen, \& Kim, 2009). These discourses formed the basis of the micro-foundations approach to studying knowledge transfers between units in multinationals (Felin \& Foss, 2005; Felin et al., 2015; Foss, 2007; Foss \& Pedersen, 2004). 


\subsubsection{Individual level knowledge transfer and individual level antecedents: Quad 3}

Some early studies of individual knowledge exchange in an organization dates back to Allen and Cohen's (1969) and Keller and Holland's (1983) studies conducted in R\&Ds. Allen and Cohen (1969) identified 'status' of the employees as an important factor impeding knowledge flows among the lower-status members. They found that communication among higher-status members was more than communication among the lower-status members. Even lower-status members tried to associate and direct their communication more towards the higher-status members than other lower-status colleagues. Keller and Holland (1983) identified personality traits, such as innovative orientation, a low need for clarity, and high self-esteem, which enhanced risk-taking and ability to innovate of communicators/innovators who were mainly responsible for knowledge creation, diffusion and transfer in the R\&Ds.

Over the past decade, studies have been appearing again, with focus on identifying antecedents to individual level knowledge transfers in multinationals. It was found that individuals' intentions to share knowledge depend on their positive attitude and motivation towards knowledge sharing (Cabrera \& Cabrera, 2005). As knowledge processes require effort, motivation is a prerequisite for employees to expend such effort (Argote \& Ingram, 2000; Minbaeva, 2013).

Researchers have examined individual knowledge transfer behaviour in the light of psychological theory of motivation, the self-determination theory (SDT) (Gagne \& Deci, 2005; Gagne, 2009; Minbaeva, 2013; Reinhol, Pedersen, \& Foss, 2011; Foss, Pedersen, Reinholt \& Stea, 2015). According to the SDT, motivation not only varies in level, but also in kind. Individual behaviour depends upon different types of motivation. Gagne (2009) differentiates between two types of motivation; autonomous and controlled. According to her, autonomous motivation is a better predictor of knowledge sharing attitude which eventually leads to knowledge sharing behaviour. When individuals are autonomously (or intrinsically) motivated to share knowledge, they volitionally engage in the sharing activity without pressure from any external sources. They pursue knowledge sharing out of their interest and passion for their work, which is more effective than engaging in sharing due to expectations of rewards or benefits.

Stenius, Haukkala, Hankonen, and Ravaja (2017) tested Gagne's model of knowledgesharing motivation and found support for autonomous motivation which came out to be the strongest predictor of knowledge-sharing intentions. Additionally, Reinhol et al. (2011) posit that having ability and opportunity to share knowledge can lead to knowledge sharing only when an employee is adequately autonomously motivated. A similar distinction between three 
motivation types; intrinsic, extrinsic and introjected, was made by Foss, Minbaeva, Pedersen, \& Reinholt (2009). Foss et al. (2009) found that intrinsic (similar to autonomous) and introjected motivation (to enhance self-worth through knowledge sharing) of an employee are positively related to the extent of knowledge sending by an employee. However, extrinsic motivation may lead to lesser knowledge sharing due to individuals sharing only the amount required to obtain the external rewards.

Studies have found that deploying HRM practices can influence individual knowledge transfer behaviour through impacting individuals' motivation to share knowledge (Minbaeva et al., 2012, Foss, Pedersen, Fosgaard, \& Stea, 2015). While some HR practices affect intrinsic or autonomous motivation, others affect extrinsic motivation of the employees to share knowledge. However, Dasí et al. (2017) found that extrinsic motivation is more important than intrinsic for inter-unit/cross-border knowledge sharing. Organizational separation and differences in contexts of separate units demands more time and effort. Also, political friction and competition between units may reduce intrinsic motivations of individuals to share knowledge across units.

Motivation or a positive attitude to seek or share knowledge also depends upon an individual's personality characteristics. Only a few studies have empirically examined the role of individual personality in knowledge sharing (Wang \& Noe, 2010). Research on personality traits have used the standard 'Big Five' traits which includes extraversion, agreeableness, neuroticism, conscientiousness, and openness to experience (McCrae \& Costa, 1989). Matzler, Renzl, Muller, Herting, and Mooradian (2008) found that individuals who have traits such as agreeableness, conscientiousness and openness are more willing and likely to engage in more knowledge sharing. In a study on knowledge sharing in the Knowledge Management Systems in organizations, Wang, Noe, and Wang (2014) found that knowledge governance practices like evaluation and reward for knowledge sharing works best with employees having higher levels of neuroticism and lower levels of conscientiousness and openness to experience. Furthermore, pro-social individuals as against pro-self or selfish are more likely to share information in group decision-making (Steinel, Utz, \& Koning, 2010).

Individuals should also have the ability to receive and share knowledge. Having expertise, aptitude, experience and prior related knowledge not only helps in acquisition of new knowledge or knowledge absorption by the individual, but also in providing and communicating the right knowledge in an understandable way (Minbaeva, 2013). Self-efficacy and openness to experience have a positive psychological effect on intensions to seek/share 
knowledge, due to which they engage more in knowledge seeking/sharing (Cabrera, Collins, \& Salgado, 2006).

Interpersonal knowledge exchange also depends upon the quality of relationship between individuals. Social ties between individuals are important conduits of knowledge transfer relationships (Cabrera \& Cabrera, 2005). Inter-unit knowledge transfer is fostered by direct inter-unit ties or building social capital between members of different units across the border (Kachra \& White, 2008; Mäkelä, Andersson, \& Seppala, 2012; Tortoriello, Reagans, \& McEvily, 2012; Zhao \& Anand, 2013). Mere existence of ties is not a sufficient condition for knowledge exchange to take place. Mutual trust between the individuals is necessary for effective knowledge sharing (Cabrera \& Cabrera, 2005; Mäkelä \& Brewster, 2009; Raab, Ambos, \& Tallman, 2014). Trust makes a knowledge source and the knowledge shared more reliable. Also, without trust on the recipient, regarding how the shared knowledge will be used, a source will be reluctant to share his/her knowledge.

According to the social exchange theory (Blau, 1964), individuals regulate their behaviour based on analysis of costs and benefits involved in undertaking the behaviour. Knowledge exchange is also a kind of social exchange where individuals will scrutinize the expected costs and the resultant benefits from seeking/receiving or sharing knowledge. The perceived expertise and the value of the knowledge held by the source is a crucial deciding factor for searching and seeking information/knowledge/advice (Borgatti \& Cross, 2003; Nebus, 2006). A lack of awareness of relevance of each other's knowledge or the tendency of "not-invented-here" syndrome (Katz \& Allen, 1982), that is, the failure to appreciate and then devalue each other's knowledge may create barriers to cross-border knowledge seeking (Haas \& Cummings, 2015). Knowledge sharing/seeking entails incurring costs related to loss of time spent on sharing/obtaining knowledge, monetary costs and effort required to reach out to the source, costs of communication, psychological cost due to requestor's display of knowledge inadequacy, or fear of losing valuable knowledge and competitive advantage (Michailova \& Husted, 2003). Therefore, expectation of reciprocity is an important factor for knowledge exchange relationships. Individuals will be encouraged and willing to share knowledge when they can expect to receive some economic benefits in the future or expect reciprocation of the behaviour when he/she needs to seek knowledge (Cabrera \& Cabrera, 2005; Caimo \& Lomi, 2015; Kachra \& White, 2008; Schrader, 1991).

Expectation of reciprocity and willingness to share knowledge is also dependent on competition between the exchange partners. Bouty (2000) posited that economic interest of the organization and the individual's personal objectives are intertwined in employee's decision 
making in whether to share a particular resource or not. If the exchange partners are direct competitors, resources available for exchange will be fewer and likelihood of exchange will be lesser. In a study of R\&D scientists' cross-unit knowledge exchange behaviour, Kachra and White (2008) found that when the level of competition between the units is higher, the scientists made smaller efforts to respond to requests for knowledge from the competing unit.

Knowledge sharing can bring status and power to the individual. Need for status can drive an individual to share knowledge and expertise. By sharing knowledge proactively, individuals try to differentiate him/herself from others and show-off their unique expertise. This helps them obtain social recognition and higher status (Park et al., 2017). However, a higher perceived power of oneself and higher self-confidence can deter willingness to seek advice from others, both experts and novices (See, Morrison, Rothman, \& Soll, 2011; Tost, Gino, \& Larrick, 2012). Sometimes employees might refrain from seeking help/advice from colleagues as help seeking implies dependence and incompetence which can lead to loss of power (Lee, 1997).

Last but not the least, individual characteristics such as age, education, tenure of the individuals in the organization, and such other demographic characteristics of the recipient and the source, are found to motivate individuals to interact with possible knowledge sources in other subsidiaries/subunits (Haas \& Cummings, 2015).

\subsubsection{Individual level knowledge transfer and organizational level antecedents: Quad 4}

Due to proximity of the factors to individuals, individual level factors can directly impact individual knowledge exchange behaviour. Nevertheless, there are factors at the organizational level which can exert their influence through effects on the individual level antecedents.

An organizational environment supportive of knowledge exchange, while also facilitating it, is necessary for more knowledge exchanges to take place between employees. Organizational values and a work climate that promotes a collaborative culture of trust and social relationships create a positive attitude and enhance motivation of employees towards knowledge sharing which positively impact their knowledge exchange behaviour (Cabrera \& Cabrera, 2005; Foss et al., 2015; Llopis \& Foss, 2016;Michailova \& Minbaeva, 2012). Roles of leaders and managers in creating a knowledge sharing work climate by motivating employees has also been highlighted (Carmeli, Gelbard, \& Reiter-Palmon, 2013; Raab et al., 2014).

In a multinational setting, the primary factor that hinders knowledge transfer between individuals located in different subunits is the geographic distance between the individuals 
(Nebus, 2006). Haas and Cummings (2015) found that geographic differences between members of the MNE located in two different countries, regions, or time zones increase communication costs, difficulty of understanding knowledge, and make knowledge exchange arduous. In addition to geographic separation, structural differences such as differences in operating division, business units, and functional areas can create concerns about relevance of knowledge due to less awareness and appreciation of each other's knowledge. Individuals sharing similarities of organizational functions also share common field-related knowledge, rely on common reference points and thus have a common shared cognitive ground between them, which facilitates knowledge sharing (Mäkelä, Kalla, \& Piekkari, 2007; Mäkelä et al., 2012). However, organizational level similarities between the operational contexts of subsidiaries/units which include culture, language, and functions help build social capital between the individuals and create a more favourable environment for knowledge exchange (Mäkelä et al., 2012).

Apart from similarity or difference in national culture, national culture in itself may influence knowledge sharing behaviour of individuals. Certain cultural traits such as pro-social, and relationship oriented can positively influence knowledge sharing. Michailova and Hutching (2006) found that a vertical collectivist culture (in-group oriented) and particularism (personal relationship oriented) can lead to intensive social relations among organizational members which facilitates more knowledge sharing within the organization. Collectivist and particularistic individuals have a tendency to differentiate between an in-group and out-group and display a strong sense of in-group oriented behaviour which can lead to more exchanges within the unit than across.

Most of the studies on organizational level antecedents come under the umbrella of the Knowledge Governance Approach (KGA) which is a crucial advancement towards opening the "black box" of micro-foundations for macro organizational knowledge transfers (Foss, 2007). According to this approach, deployment of governance mechanisms, which are organizational level factors, influence knowledge processes through their influence on the conditions of individual action (Foss, 2007; Gooderham, Minbaeva, \& Pedersen, 2011). KGA has been used by a number of studies to examine individual level conditions of individual knowledge exchange behaviour and draw inferences to understand knowledge transfers at the organizational level (Minbaeva, 2008; Minbaeva et al., 2012).

KGA identifies both formal and informal mechanisms at the organizational level. Informal mechanisms involve creating an organizational culture or a value system that creates social capital and encourages and facilitates knowledge sharing among employees. Formal 
mechanisms include HRM practices such as job design, rewards and incentives, training and development, which impact an individual's ability and motivation to share knowledge (Minbaeva, 2013; Morris, Zhong, \& Makhija, 2015). Practices like performance appraisal, incentives etc. can impact an individual's motivation to engage in knowledge sharing (Gagne, 2009; Foss et al., 2015). Some practices like collaborative work design increase interaction between individuals that aid knowledge transfers (Kaše, Paauwe, \& Zupan, 2009). Informal mechanisms like deploying social governance mechanisms help in establishing psychological contracts with the employees to create trust, commitment and goodwill for the organization and motivate employees to engage in knowledge exchanges (Gooderham et al., 2011). Similarly, commitment-based mechanisms, which use cognitive stimulation, are better drivers of knowledge transfer behaviour than transaction-based mechanisms, which use tangible and explicit incentives for promoting knowledge sharing behaviour (Husted, Michailova, Minbaeva, \& Pedersen 2012).

\subsection{Knowledge Governance Approach}

Organizations use governance mechanisms to influence individual behaviour to achieve organizational objectives. The Knowledge Governance Approach propounded by Foss (2007) explores how the deployment of governance mechanisms at the organizational level influences knowledge processes, such as creating, sharing and retaining knowledge in the organization. It is known that the formal aspects of organization such as organization structure, work design, coordination mechanisms, training and development or reward systems can be manipulated by the organization (Grandori, 2001). The deployment of governance mechanisms can influence and change individual behaviour related to the knowledge processes. The KGA takes a micro behavioural view and posits that the organizational level governance mechanisms exert their influence on the organizational knowledge processes through their impact on actions and behaviours of individual actors. It explores the underlying mechanisms at the individual level through which the organizational level processes operate. Doing so, it makes explicit behavioural and cognitive assumptions about an individual actor's/agent's perceptions, beliefs, motivation and preferences in a knowledge sharing/exchange context (knowledge sharing/exchange process being the focus of this discourse).

Similar to the transaction cost economics logic as applied to knowledge transactions, the objective of KGA is minimizing the cost and maximizing the net benefits from the knowledge processes as well, more specifically, knowledge exchange/sharing through deploying the governance mechanisms (Foss, 2007; Heiman \& Nickerson, 2002; Williamson, 1985). But 
KGA, unlike the transaction cost economics, makes the underlying behavioural and cognitive assumptions in a knowledge sharing context very explicit (Foss, Husted, \& Michailova, 2010). The KGA draws on Coleman's bath tub model (Figure 2.2) (Coleman, 1990; Foss, 2007; Minbaeva et al., 2012) to explicate how the organizational level antecedents affect organizational level outcomes of knowledge transfer through impacting the conditions of individual action of knowledge sharing/exchange. According to Coleman (1990), organizational antecedents (macro) influence the "conditions of individual behaviour" (micro) (Arrow 1), which, along with other individual factors, affect changes in individual behaviours (micro) (Arrow 2). These individual actions aggregate and lead to favourable organizational level outcomes (macro) (Arrow 3). Arrow 4 depicts the macro to macro relationship when organizational level outcomes are explained by organizational/macro-level phenomena.

Figure 2.2 Interaction of knowledge transfer and its antecedents at two levels-individual and organizational

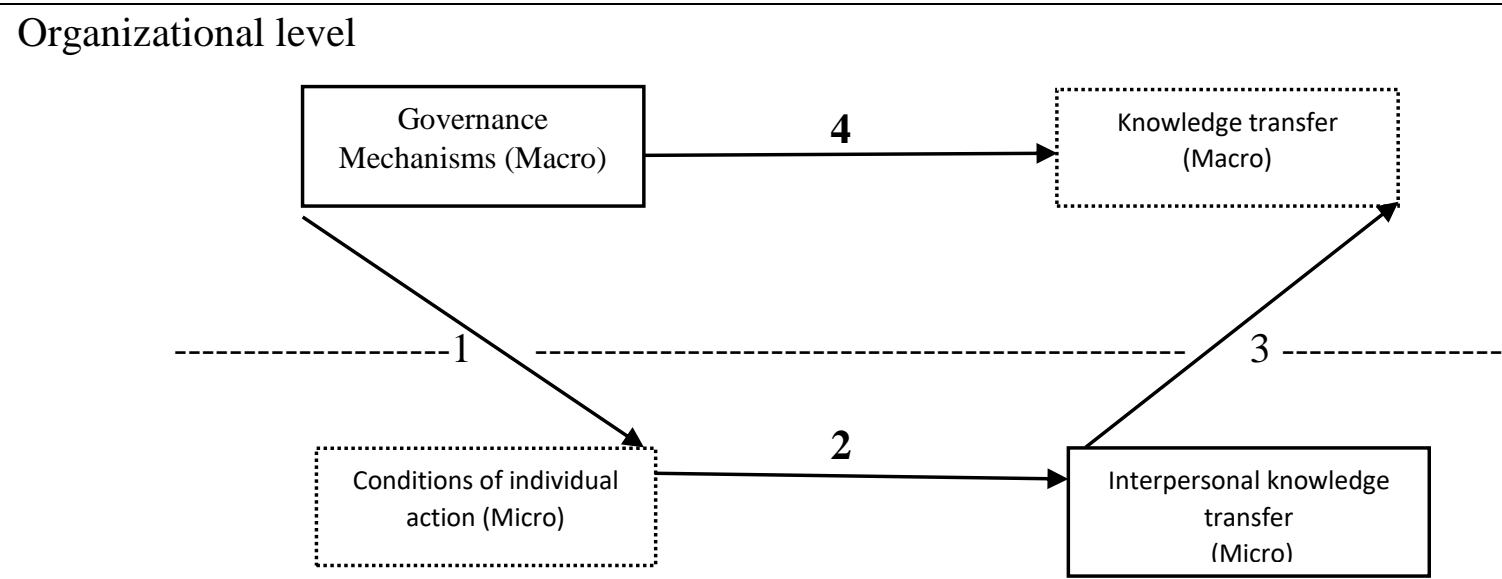

Individual level

Source: Foss (2007); Minbaeva, Makela and Rabiossi (2012), adapted from Coleman (1990).

Foss (2007) adapted Coleman's (1990) analysis to explain the multilevel nature of the Knowledge Governance Approach to knowledge transfer by integrating and examining the interplay between antecedents at the two levels-organizational/macro and individual/micro. Using the bath-tub, Foss (2007) has broken down the underlying knowledge transfer process by exhibiting how the governance mechanisms at the macro level have a psychological or cognitive influence on the individual (micro level) (Arrow 1), which motivates them to engage in knowledge sharing/exchange (Arrow 2). The conditions of individual actions are an individual's perceptions, attitudes, desires, assessment and such other cognitive effects which lead them to engage in knowledge exchange behaviour. Consequently, the integration of these 
individual knowledge exchange behaviours would lead to organizational level macro knowledge transfers.

Foss et al. (2009) and Foss et al. (2010) posit that the organizational level antecedents of knowledge sharing involve both formal and informal factors. Formal aspects are the organizational structure, goal setting, directives, rules and regulations (Foss et al., 2010; Grandori, 2001) and the informal aspects relate to trust, networks, social ties, culture, identity and identification (Argote \& Kane,2009; Michailova \& Husted, 2003).

Thus, KGA is the choice, combination, and deployment of formal and informal organizational mechanisms to influence individual knowledge sharing behaviour in organizations so that organizational knowledge-based goals (knowledge transfer, building absorptive capacity, capabilities and obtaining competitive advantage) can be achieved (Foss et al., 2010, p 459). Both formal and informal mechanisms are crucial to the understanding of knowledge sharing (Foss et al., 2010, p. 470).

\subsubsection{Formal mechanisms}

Out of the formal organizational antecedents, human resource management practices have been researched most extensively. Particularly because these are direct governance mechanisms which can be deployed to influence individual knowledge sharing behaviour. HRM practices such as job/work design, incentives and rewards, training and development can impact individual knowledge sharing behaviour. HRM practices have an influence on three antecedents of individual knowledge sharing behaviour-ability, motivation and opportunity (Minbaeva, 2013).

Collaborative work design such as job rotation and cross-functional teamwork increase social interactions and increase knowledge exchanges through improving interpersonal relationships (Kaše et al., 2009). Job characteristics such as autonomy, task identity and feedback can foster different types of individual motivation; intrinsic, introjected, and external respectively (Foss et al., 2009). When employees are intrinsically motivated, they find the job interesting, enjoying and stimulating. They become proactive and constantly strive to promote their personal growth through learning and such other positive activities like knowledge sharing.

Likewise, introjected motivation leads employees to engage in knowledge sharing behaviour that puts them in a positive light due to meeting the organization's expectations. Also, feedback in the form of performance evaluations and recognition increases an employee's external motivation leading to knowledge sharing behaviour by sending signals to the 
employee that knowledge sharing is important and valued by the organization (Foss et al., 2010). As found by Foss et al. (2015), employees have a higher autonomous motivation to engage in knowledge sharing when they have autonomy and variety in their job.

Similarly, HR practices like performance appraisal, rewards, incentives, training and development, work climate, and managerial styles can promote an individual's autonomous motivation to engage in knowledge sharing by satisfying psychological needs of attitude, need satisfaction, and sharing norms (Foss et al., 2015; Gagné, 2009). Morris et al. (2015) found that when outcome-based incentives are used, employees are more motivated to search for and reach out to distant knowledge in the organization. Likewise, training and development satisfies psychological needs of the sharing norm and enhances relationships among individuals (Gagné, 2009; Kaše et al., 2009).

\subsubsection{Informal mechanisms}

Among the informal mechanisms is organizational culture which promotes employee knowledge sharing. Organizational values, a key component of organizational culture, helps an organization frame the standards and criteria to establish the organizational objectives and choose the courses of actions to achieve those set goals. Michailova and Minbaeva (2012) found that espousing, enacting and internalizing the value of dialogue as a core organizational value facilitates knowledge sharing among organizational members (p. 67). They posit that organizational values affect employees at both cognitive and behavioural levels. At the cognitive level, a pro knowledge sharing value can positively influence an individual's attitude and basic assumptions towards knowledge sharing, which then shape individual knowledge sharing behaviour.

Likewise, a cooperative climate can foster knowledge sharing in the organization (Quigley, Tesluk, Locke, \& Bartol, 2007) as it is conceived of as a descriptive norm requiring members to behave in a cooperative manner. As found by Llopis and Foss (2016), a cooperative climate can serve as a supplementary source of motivation for employees who lack any intrinsic motivation towards engaging in knowledge sharing. Also, to strengthen the positive effect of cooperative climate on knowledge sharing, they suggested that job autonomy should be provided.

The importance of social capital on knowledge transfer behaviour has been highlighted by Bouty (2000), Gooderham et al. (2011) and Tsai (2002). Networks crossing organizational boundaries and ties between individuals facilitate individual information searches across the organization leading to increased knowledge sharing (Cross \& Cummings, 2004). However, to 
facilitate increased knowledge exchange among individuals, it is important that in addition to creating networks, individuals occupy a central position in the network and possess both autonomous motivation and ability to acquire and provide knowledge (Reinholt et al., 2011).

Moreover, not only the organizational culture, but also national culture can impact individual knowledge sharing behaviour in the organization. In a comparative study of individual knowledge sharing behaviour in Chinese and Russian organizations, Michailova and Hutchings (2006) found that a vertical collectivist culture (in-group oriented) and particularism (personal relationship oriented) can lead to intensive social relations among organizational members which then facilitates more knowledge sharing between the in-group members.

Some studies contend that informal organizational antecedents exert their influence only through the underlying mechanism of influencing individual perception. As Gooderham et al. (2011) suggests, knowledge governance mechanisms operate in a multi-level context. They exert their influence through the individual experiences or perceptions of these mechanisms. As suggested by Kehoe and Wright (2013), perceived HR practices (practices that are perceived and interpreted subjectively by each employee) are temporally closer to, and consequently likely to be more predictive of individuals' attitudinal and behavioural outcomes (p. 4). According to Minbaeva (2013), perceived HR practices, measured at the individual level, are more predictive of individual knowledge sharing behaviour. Employees' perceptions of their organization's commitment to knowledge sharing and their perceived intensity of training, promoting their personal growth, can intrinsically motivate individuals to engage in social interactions and lead to increasing knowledge sharing behaviour (Kuvaas, Buch, \& Dysvik, 2012; Minbaeva et al., 2012). Thus, as posited by Minbaeva (2013), HRM practices influence an employee's ability, motivation, and opportunity to engage in knowledge sharing which leads to their knowledge sharing behaviour.

Another informal mechanism, which bears similarity with the theory that has been used in this study, is identity and identification. Argote and Kane (2009) contend that members belonging to different units in an organization share a sense of belonging to a higher-order unita superordinate social identity, from which they derive a portion of their identity. This can act as a coordination and governance mechanism that can influence knowledge sharing/transfer between the units. This is more of a relational approach, which is distinct from the contentbased approach, which conceptualizes organizational identity based on distinctive features of the organization (Argote \& Kane, 2009). 


\subsection{Points of Departure and the Research Gap}

This summary of literature review (Figure 2.1) indicates that rich literature exists when knowledge transfer and its antecedents are at single level: organisational factors to organisational knowledge transfer (Quad 1) and individual level factors to individual level knowledge exchanges (Quad 3). Recent research is emerging for examining multi-level knowledge transfer processes (Quad 2 and Quad 4): Individual antecedents impacting organizational transfers (Quad 2) and organizational antecedents impacting individual transfers (Quad 4).

A need to understand individual knowledge transfer behaviour is gaining importance as it is crucial to understanding knowledge transfer at the organizational level, which is the central tenet of the micro-foundations approach (Felin et al., 2015). Quad 3 is more explored than Quad 4 as it is informed by studies in various other disciplines, primarily in psychology, organizational behaviour, and sociology. As a multilevel phenomenon, Quad 4 provides an opportunity to examine individual behaviour in the larger context of an organization and more so in the context of a multinational organization having distinguishing characteristics of diverse local and global organizational factors exerting influence. Most of the current studies are under the KGA, which explores factors or mechanisms deployed to govern individual knowledge exchange behaviour. Through a focus on Quad 4, I aim to bring in a set of organizational level factors, unique to an MNE, and add to the KGA.

\subsubsection{Importance of individual level knowledge transfer}

A review of the above literature reveals that most of the studies on intra-MNE knowledge transfer examine transfers at the organizational level (Gupta \& Govindarajan, 2000; Michailova \& Mustaffa, 2012; Minbaeva et al., 2003; Schulz, 2003). At the micro level, knowledge transfer takes place between individual actors (Argote \& Ingram, 2000). It is observed that in the last two decades there has been a clarion call for exploring microfoundations of organizational processes such as knowledge transfer. In response to this, various researchers have attempted and successfully been able to produce seminal works both theoretical and empirical (Borgatti \& Cross, 2003; Dasí et al., 2017; Haas \& Cummings, 2015; Makela \& Brewster, 2009; Michailova \& Husted, 2003; Minbaeva et al., 2012; Zhao \& Anand, 2013). Most of these are about individual knowledge sharing/exchanging/providing/sourcing behaviour within the organization or across subunits/departments within an organization in a specific country context. These studies are mainly found in the HRM discourse. However, relatively little attention has been focused on cross border knowledge transfer between 
individuals in the MNE, who belong to subsidiaries located in different countries (Foss et al., 2010; Foss \& Pedersen, 2004). As aptly put by Makela et al. (2007), "the issue of who interacts with whom is of fundamental importance to how knowledge flows internally, but has thus far received surprisingly little attention in the literature concerning multinational corporations" (p.2).

Performance and success of a multinational depends upon the integration and transfer of its knowledge across its geographically dispersed subsidiaries (Argote \& Ingram, 2000). Knowledge transfer within the multinational across nations has been linked to innovation (Kotabe et al., 2007; Phene \& Almeida, 2008), better coordination (Szulanski, 2000) and to its competitive advantage (Grant, 1996; Kogut \& Zander, 2003). As the knowledge-based view suggests, individual actors responsible for storage, creation, and transfer of knowledge are the locus of knowledge in the organization (Grant, 1996). As suggested by Caimo and Lomi (2015), they help finding solutions to complex problems that require integratios of different knowledge and expertise located across different units.

Interpersonal knowledge exchanges may be both formal and informal. Informal exchanges are purely interpersonal, ad hoc, and independent of organizational structure, policy, and formal collaborations (Allen, 1977). They are outside the purview of any organizational directives or surveillance. Formal exchanges are the ones which are required for completion of a task, where the knowledge sources are prescribed or identified by the organization. As put by Nebus (2006), it is not uncommon for an individual to be assigned a task without prescribed paths to complete the task. For these kinds of non-routine, complex tasks, individuals search for advice and knowledge to leverage the knowledge, expertise and experience of others. These are called informal as there are no prescribed steps to complete the task and the individual himself/herself can search for knowledge informally. This applies to situations where the seeker has the freedom of choice with whom to contact for knowledge. Task advice relations are similar to such informal relations (Nebus, 2006). Appleyard (1996), Kachra and White (2008), and Schrader (1991) provide evidence of such informal knowledge exchanges where R\&D scientists provide technical information or advice to colleagues as they regularly receive requests for know-how from other R\&D scientists, both inside and outside their own firms. Mäkela et al. (2007) as well explored informal individual knowledge sharing in the MNC context.

The informal knowledge exchanges are mediums of transferring critical information. Research scientists exchange very crucial proprietary knowledge and information across organizational boundaries through these informal resource exchanges (Bouty, 2000; Hippel, 
1987; Kreiner \& Schultz, 1993). It was found that personal contacts of scientists with people external to the firm accounted for about $40 \%$ of the ideas generated in the firm (Allen, 1977). In an empirical study of Danish and US subsidiaries, Schulz (2003) found that informal relations between units have a positive effect on inflows of knowledge to the focal subsidiary from a peer or supervising unit. Morris et al. (2015) also found that the more the employees reach out to distant knowledge outside their unit within the MNE, the more their project performance increases. Thus, individual knowledge transfers across the border are crucial for the MNE.

However, individuals as well as their behaviours are heterogeneous (Felin \& Hesterly, 2007). An individual employee's behaviour is independent and many times it does not align with the goals of the organization (Bjerregaard \& Klitmoller, 2016). These interpersonal knowledge exchanges vary in frequency and are asymmetric as an individual may seek knowledge from another without the reverse taking place (Haas \& Cummings, 2015; Kilduff \& Krackhardt, 1994). Also, Mäkela et al. (2007) contend that informal knowledge sharing/exchange in an organization displays an uneven pattern as individuals have a tendency to interact and share knowledge more with similar others. As put by Steinel et al. (2010), individual knowledge sharing is a mixed motive and strategic behaviour in nature. The drivers of this behaviour are both at the personal level, at the level of the individual, and at the organizational level. The factors that influence individual knowledge sharing across organizational units are distinct and different from the ones that promote knowledge sharing within the organization (Dasí et al., 2017). Therefore, examining individual cross-border knowledge exchanges is not only crucial to understand knowledge transfers at the organizational level, but also provides us insights into cross-border behaviour of individual employees in an MNE, that transcends geographical, organizational, and cultural boundaries.

\subsubsection{Individual level knowledge transfers in MNEs-filling the research gap}

The pioneers of the recent surge in individual level knowledge sharing research are Borgatti and Cross (2003), Bouty (2000), Cabrera and Cabrera (2005), Cabrera et al. (2006), Husted and Michailova (2002), Mäkela at al. (2007), and Michailova and Husted (2003). Out of the recent lot were the ones who examined interpersonal cross-border knowledge exchanges in MNEs. However, Mäkela et al.'s (2007) work was the first to more explicitly focus on the cross-border contextual aspects that may influence individual knowledge sharing in the MNE. Although there have been a good number of studies on individual knowledge sharing in the 
organization, cross-border individual level knowledge sharing in an MNE is still less explored (Dasí, et al., 2017).

Earlier, Ambos and Ambos (2009); Gupta and Govindarajan (2000); and Hansen and Lovas (2004) have pointed out organizational separation in the MNE as a factor hindering organizational knowledge transfers. Dasí et al. (2017) examined individual knowledge sharing behaviour under two different contexts, within the same business unit located in the same national context, and across business units located in different national contexts. They found that individual knowledge sharing behaviour in MNEs is influenced by variation in the organizational context which has largely been ignored. Internal boundaries in an MNE due to organizational separation between business units affect individual knowledge sharing behaviour. The effect of organizational separation in MNEs is "distinct from (and additional to) the various types of 'distance' that are found to be affecting knowledge sharing, as identified by the IB literature" (Dasí et al., 2017, p. 432). This calls for the need to identify those factors that emerge as a result of organizational separation of the business units in the MNE which may hinder the individual knowledge exchange behaviour across business units.

MNE business units (not functional or departmental units) or subsidiaries, as generally called, are units of the same MNE sharing the same strategic mission and larger MNE goals, and connected through interdependencies created due to similarity of operations; but, having a distinctly separate and defined profit-loss responsibility (Dasí et al., 2017; Govindarajan \& Fisher, 1990; Gupta \& Govindarajan, 1984). Rather than a "unitary organization", an MNE is an "inter-organizational network" of subsidiaries embedded in a network of customers, suppliers or such other external actors (Ghoshal \& Bartlett, 1990). Thus, every subsidiary has a different role and its relationship with the HQ varies accordingly. Subsidiaries are semiautonomous units with independent objectives and they differ in terms of their mandates, responsibilities and functions (Ambos, Andersson, \& Birkinshaw, 2010; Birkinshaw \& Hood, 1998).Depending on their roles, resources and activities, subsidiaries occupy different positions of importance in the development of the multinational and its performance (Wernerfelt, 1984). Based on their strengths and weaknesses, they exert considerable influence on the strategic decisions of the multinational, and also have more bargaining power in the distribution of MNE resources among the subsidiaries (Ciabuschi, et al., 2012; Mudambi \& Navarra, 2004). Thus, subsidiary power plays a crucial role in subsidiary evolution through charter change (Balogun, Jarzabkowski, \& Vaara, 2011; Birkinshaw \& Hood, 1998; Dörrenbächer \& Gammelgaard, 2006), in subsidiary initiative taking and issue selling (Dörrenbächer \& Gammelgaard, 2016). 
These power dynamics in the MNE also influences managers' and employees' behaviour in a subsidiary. Ciabuschi et al. (2012) and Mudambi and Navarra (2004) found that managers in a high-power subsidiary engage in rent-seeking behaviour. Also, high power HQ employees are found to impose knowledge and practices on low-power subsidiaries as they assume that the knowledge held by the low-power is inferior to theirs (Frenkel, 2008). Further, managers in subsidiaries are found to hide crucial information or underlying problems from the high power HQ to avoid adverse reaction or to further their position in the organization (Bjerregaard \& Klitmoller, 2016; Whittle et al., 2016).Therefore, it is likely that cross-border individual knowledge sharing behaviour is influenced by subsidiary power.

I use the Social Identity Theory (Tajfel, 1978; Turner, 1975) as the underlying theory that explains the relationship between subsidiary power and individual knowledge transfer. Subsidiary power is an organizational level factor. Through bringing in a set of 'identity' based organizational level antecedents, I would like to contribute to governance of knowledge in the organization, the KGA. The conceptual development is discussed in the next chapter. Further, two boundary conditions are added to the above relationship. One is the intra-MNE competition between the subsidiaries and the other is the cultural intelligence of the individual employee. Competition within the MNE specifically between the subsidiaries is likely to influence the relationship between subsidiary power and knowledge transfer through identification. This relationship will also be influenced by the cultural intelligence of employees.

\subsection{Subsidiary Power}

Power, in common parlance, means the ability to control the behaviour of others. Power manifests in the possession of authority, control or influence over others. It plays an important role in social, political and economic interactions (Rucker \& Galinsky, 2008). Controversy regarding the conceptual definition of "power" is not new; and a review of literature shows that the controversy has not been resolved (Pfeffer, 1981; Provan, 1980).

Weber's (1947) definition has provided the initial foundation for the concept of power. He defined power as the probability of a man or a number of men to realize his/their own will inspite of resistance from others. Dahl (1957) defined power in terms of the relationship between people. According to him, the ability of A to influence B is that power of A over B. Emerson's (1962) definition highlights a dependency relationship, where, A is more powerful than B if B is dependent on A for scarce and valuable resources. Diverting from the relationship perspective, Mechanic (1962) interprets power as a force which results in changes in individual behaviour (in the absence of which the behaviour would not have changed). Magee and 
Galinsky (2008) indicated that existence of power does not always require changing behaviours of actors. Even a passive presence of a powerholder might lead to changes in an individual's behaviour. Power has also been discussed with respect to whether it is enacted or potential (Provan, 1980). While potential power is the "capacity to influence future outcomes", enacted power is the "actual exercise of power" (Provan, 1980, p. 550).

The concept of power was initially studied at the community level and later at the level of the work organization. In the organization, power has been studied at both the interpersonal and organizational levels. Interpersonal level power involves work on the power of managers, subordinates, and top management team; whereas, organizational level involves power of subunits and departments (Feng, Morgan, \& Rego, 2015; Fiol, O'connor, \& Anguinis, 2001; Galang \& Ferris, 1997; Schein, 1977; Sivanathan, Pillutla, \& Murnighan, 2008; Treadway et al., 2013; Turner \& Schabram, 2012). At the interpersonal level, the most popular typology of a power base was put forward by French and Raven (1959). They identified five bases of interpersonal power; reward power, legitimate power, referent power, expert power and coercive power. At the organizational level, the strategic contingencies' theory of intraorganizational power by Hickson et al. (1971) attributes power of the subunit to its ability to cope with uncertainty, substitutability of its coping activities and centrality of its functions, through control of strategic contingencies for the dependent activities. According to Salancik and Pfeffer (1974), a subunit will possess power when it provides organizations with resources, which are considered to be critical, important and valuable for the organization.

According to the Resource Dependency Theory, power of a unit depends on the resource dependency relationship it has with other units (Medcof, 2001; Pfeffer \& Salancik, 1978). The size of a unit was also found to be highly correlated with power. Large size units are usually more powerful than the smaller units as their size is likely to affect innovation and performance of the unit due to greater resource allocation (Tsai, 2010). While various studies have tried to attribute intra-organizational power to resource dependency and critical scare resources, Astley and Sachdeva (1984) interpreted power in terms of properties of the organization, the "structural" sources of power. They opined that intra-organizational power is the result of interaction between three sources of power: hierarchical authority, resource control and network centrality.

In a multinational organization, the concept of subsidiary power rests in the view of the multinational as a heterarchical, network structure (Bartlett \& Ghoshal, 1989; Hedlund, 1986) rather than a hierarchical structure. Ghoshal and Bartlett (1990) were of the opinion that as subsidiaries within an MNC have different roles and the HQ-Subsidiary relationships vary 
accordingly, the MNC is far from being a "unitary organization". Rather, it can be conceptualized as an "inter-organizational network" where the units are embedded in a network of customers, suppliers or such other external actors. Subsidiaries differ in terms of their mandates, responsibilities and functions (Birkinshaw \& Hood, 1998). They are semiautonomous units with independent objectives (Ambos et al., 2010).

As Resource Based Theory suggests, firm level resources which are valuable, rare and non-substitutable, provide the firm with unique competitive advantages and become the basis for power (Peteraf, 1993; Wernerfelt, 1984). Depending on their roles, resources and activities, subsidiaries occupy different positions of importance in the development of the multinational and its performance. Based on their strengths and weaknesses, they can exert considerable influence on the strategic decisions of the multinational. Subsidiary power, thus, may refer to a subsidiary's ability to influence its parent's decision in strategic and operational activities (Dörrenbächer \& Gammelgaard, 2006). While one of the dimensions of subsidiary power is having influence over the MNC's strategic decisions, the other dimension is having the freedom to pursue its own goals, that is, autonomy (Ambos et al., 2010; Najafi-Tavani, Zaefarian, Naudé, \& Giroud, 2015). Some researchers also use the term bargaining power to refer to power of the subsidiary (Ciabuschi et al., 2012; Dörrenbächer \& Gammelgaard, 2011; Mudambi \& Navarra, 2004).

Dörrenbächer and Gammelgaard (2011) identified four sources of subsidiary powersystemic, resource dependency, institutional, and micro-political bargaining power. A subsidiary responsible for a specific function which is critical to the proper functioning of the value-chain network in the MNE provides it with systemic power. Power can also be derived from the host-country institutional structures. Another source is micro-political bargaining power, which is derived from a subsidiary exerting influence on the HQ through strategic information politics or other forms of manipulative behaviour. Moreover, resource dependency is also a source of subsidiary power. It is derived from a subsidiary's control over critical resources that is valuable to the MNE. Further, Mudambi et al. (2014) added to the resource dependency power and differentiated between functional and strategic power. According to them, while a subsidiary can have functional power due to technology and/or business related competencies in a particular functional domain, strategic power or its ability to influence MNE strategic decisions will be gained only when that function is critical to the MNE's performance and the MNE is highly dependent on it.

Mudambi and Navarra (2004) found that due to possession and control of the firm's R\&D knowledge, subsidiaries engage in knowledge flows from the subsidiary to other parts of the 
MNE leading to subsidiaries gaining bargaining power. Later Ciabuschi et al. (2012) and Najafi-Tavani et al. (2015) found support for this view by establishing that greater technology transfer competence of a subsidiary or its ability to engage in reverse knowledge transfers to other parts of the MNE makes it powerful within the organization. Some researchers have underscored the importance of the local business networks and subsidiary's past initiative taking, to autonomy and influence of the subsidiary in the MNE (Ambos et al., 2010; Andersson, Forsgren, \& Holm, 2007; Andersson \& Pahlberg, 1997).

Subsidiary power has also been attributed to its duration of operation or age, and its control over its production processes (Mudambi \& Navarra, 2004). Another perspective of power in the MNE has been put forward by Frenkel (2008), who opines that a subsidiary can draw power from the development status of the host nation. Ambos, Asakawa and Ambos (2011) contend that the embeddedness of a subsidiary within the internal MNE network would help a subsidiary gain higher autonomy in the future. Being part of a close-knit internal network with the HQ and allowing sufficient HQ to control would help a subsidiary build trust which eventually can lead the HQ grant autonomy to the subsidiary.

The power of a subsidiary can have a significant influence over various MNE processes which impact the subsidiary's development as well as its survival in the MNE. A powerful subsidiary can gain headquarters' attention, which is considered to be a scarce and critical resource for the development of a subsidiary (Bouquet \& Birkinshaw, 2008b). A subsidiary's evolution through role development or charter change depends on how much influence or power the subsidiary has over headquarters' decisions (Balogun et al., 2011; Birkinshaw \& Hood, 1998; Dörrenbächer \& Gammelgaard, 2006) to affect a positive change. Dörrenbächer and Gammelgaard (2016) propounded that when the headquarters-subsidiary power relations are highly asymmetrical, a greater degree of issue selling is required by the subsidiary to obtain an approval from the headquarters for the initiative it undertakes. Therefore, according to Bouquet and Birkinshaw (2008a), low power subsidiaries engage in various strategies or tactics such as profile building, deference, feedback-seeking, coalition building etc. to gain more power in the MNE.

Considering the importance of power to the subsidiary for various MNE processes, managers' as well as employees' behaviour in the subsidiary is influenced by the power of the subsidiary. It is known that subsidiaries compete to achieve a power position through which it can influence the HQ in its critical decision making which impacts worldwide operations (Luo, 2005). Dörrenbächer and Gammelgaard (2006) found that HQ's realised strategies in subsidiary role development is the result of its intended strategies modified by micro-political HQ- 
Subsidiary negotiation processes. Managers of a high-power subsidiary, with respect to its ability to bargain for internal resources within the MNE, have been found to engage in rentseeking behaviour (Ciabuschi et al., 2012; Mudambi \& Navarra, 2004).

For the purpose of this study, following Dörrenbächer and Gammelgaard (2006), Ambos et. al. (2010) and Najafi-Tavani et al. (2015), power of a subsidiary in the MNE is defined as its influence on the strategic decisions of the HQ and its autonomy to pursue its own objectives. This power may be based on various factors as identified by the studies above. This may range from the resources possessed by the subsidiary which are critical for the MNE, dependence of the HQ on the subsidiary's resources, network centrality of the subsidiary or the importance of its mandate, its size and age, importance of the market it is located, past initiative taking, development/economic status of the country in which it is located or having people at the top management who can or have influence over the HQ.

Another concept that is similar to 'power' is 'status'. Status is the extent to which someone/something is respected or admired by others (Magee \& Galinsky, 2008). The concepts of 'power' and 'status' are strongly related and they covary (Kilduff \& Galinsky, 2013). While high status makes it easier to gain power, high power often leads to gaining respect and admiration, or status. But, 'power' and 'status' are two distinct concepts (Anicich, Fast, Halevy, \& Galinsky, 2016). Sometimes power may exist without status, as in the case of a reimbursement clerk, at other times status may exist without power, as in the case of an emeritus professor (Anicich et al., 2016; Fragale, Overbeck, \& Neale, 2011). This study focuses on power, specifically subsidiary power, and its impact on interpersonal knowledge transfer.

\subsection{Intra-MNE Competition}

Competition is a behavioural outcome as a consequence of the individual's struggle for achieving high status (Liening, Mehta, \& Josephs, 2012). Usually the ones who are high in dominance are successful in achieving high status positions. Competitors are actors who share interest in the same resources (Ingram \& Yue, 2008). Limited availabilities of resources creates conditions of conflict among individuals/groups. Competition also exists between subunits in an organization. Chen (1996) identified resource similarity as an important condition for the existence of competitive tension. Internal competition is usually due to limited resources or overlaps of subunit charters (Birkinshaw \& Lingblad, 2005). Charters are the business responsibilities of the subunits entrusted by the parent (Galunic \& Eisenhardt, 1996).

The dynamics of competition can be extended to the MNE which is viewed as a "network" of differentiated units with resources (Ghoshal \& Bartlett, 1990; Gupta \& 
Govindarajan, 1991). Studies on intra-MNE competition has explored competition vis-à-vis cooperation between subsidiaries which is termed 'coopetition' (Chiambaretto, Massé, \& Mirc, 2019; Luo, 2005; Tippmann, Scott, Reilly, \& O’Brien, 2018). In an MNE setting, subsidiaries must cooperate to meet greater organizational goals but at the same time compete among themselves.

A majority of the existing research on intra-MNE competition involves studies exploring sources of competition within the MNE. Cerrato (2006) views the MNE as an internal market system, where the subsidiaries participate in three different internal markets: The first is for goods and services; the second for charters; and the third for competencies and practices. Becker-Ritterspach and Dörrenbächer (2011) viewed intra-MNE competition as competition of subsidiaries for parent's resources, mandates, system position and customers. Subsidiaries compete for limited headquarters' resources like capital, technology, knowledge, information, and corporate support to reduce dependence on the indigenous resources and strengthen their local competitive strength (Luo, 2003). They also compete to defend their system position or improve it. System position is the strategic role and position of the subsidiary in the MNE network/system. It involves competing for a position in the value chain and knowledge flow system, competing to be the centre of excellence or to have an influence over the headquarters' decisions (Luo, 2005).

Another aspect of inter-subsidiary competition is around the subsidiary mandates or charters (Tippmann et al., 2018). According to Birkinshaw (1996) charters are temporary with respect to both time and content. He argued that the subsidiary can gain a mandate based on its distinctive capabilities, but might lose it as well if it fails to continue to have supportive capabilities. In a study of a Hungarian subsidiary of Siemens telecommunication, Dörrenbächer and Gammelgaard (2010) found how a weak resource position due to diminishing attractiveness of the host country and a low network density of the subsidiary led to the removal of its charter. Thus, evolution of a subsidiary with respect to mandate/charter change, by gaining a new mandate, or developing an existing one gives rise to competition between the subsidiaries.

Research on the determinants of intra-firm competition in the MNEs has found various environmental as well as organizational factors which trigger competition between the subsidiaries. Birkinshaw and Lingblad (2005) suggest that intra-firm competition is stronger in mature and homogeneous industries. Competition for internal resources between the subsidiaries is stronger when the host country environment is unstable and requires high subsidiary responsiveness (Luo, 2005). Granting more autonomy to a subsidiary triggers 
competition as subsidiary managers predominantly act towards the interest of the subsidiary and contest for mandates or charters (Birkinshaw \& Lingblad, 2005). Weakening of a subsidiary's capabilities and loss of its competitive advantage will also instigate more competition (Birkinshaw \& Lingblad, 2005) Moreover, close headquarters-subsidiary relationships may also spur intra-firm competition between the subsidiaries (Cerrato, 2006).

Similarly, Becker-Ritterspach and Dörrenbächer (2011, p. 534) provide a political perspective and suggest that intra-firm competition has an "intrinsic conflict potential". They propose that intra-firm competition at the macro-level not only impacts HQsubsidiary/subsidiary-subsidiary relationships at the meso-level, but also negotiations among the subsidiary managers at the micro-level.

Subsidiary managers play a very crucial role in inter-subsidiary competition. BeckerRitterspach and Dörrenbächer (2011) identified subsidiary managers as important actors in intra-firm competition as they can mobilize resources both within and outside the multinational based on their resource exchange relationships. These managers act as the boundary spanners, occupy crucial positions in the network nodes, forming links between external and internal networks. Due to the pressure of intra-firm competition, subsidiary managers continuously strive to improve their bargaining power by employing the resource exchange relationships in the interest of their own subsidiary (Gammelgaard, 2009). Additionally, they may also show varying commitments to different coalitions. Headquarters and the subsidiary executives become the main "political brokers" (March, 1962, p. 672) in intra-firm competition. Friedkin and Simpson (1985) and Pfeffer and Salancik (1974) also found that due to competition among the subunits, member' identification with the subunits increased, and subunit managers encouraged resource allocation criteria that discriminately favoured their own subunits.

In a recent study on cross-functional knowledge sharing, Nguyen et al. (2018) explored the effect of cross-functional competition on formal and informal coordination mechanisms. When there is cross-functional competition, employees may be motivated to engage in more formal knowledge exchanges with the competing department in order to be aware of the activities of the competitor and be prepared for competition. But, informal knowledge exchange relationships fail to thrive due to competition as loyalty to the department takes precedence over relationships which are then marred by mistrust, hesitation, and uncertainty. Chiambaretto et al. (2019) found that sub-units face a knowledge sharing/protecting dilemma. While the subunits are required to cooperate and engage in knowledge sharing to achieve company-wide objectives, they may hesitate to do so in order to protect their idiosyncratic 
knowledge which can provide them with competitive advantage in internal competition. Thus, Intra-MNE competition hinders knowledge sharing between sub-units.

\subsection{Cultural Intelligence}

The concept of Cultural Intelligence (CQ) was first introduced by Earley (2002) and Earley and Ang's (2003). "Cultural intelligence refers to a person's capacity to adapt to new cultural settings based on multiple facets including cognitive, motivational and behavioural features" (Earley, 2002, p. 271). This definition evolved during the early stages of initiation and later, Thomas et al. (2008) built on Earley (2002) or Earley and Ang's (2003), to present CQ as a "system of interacting knowledge and skills, linked by cultural metacognition, that allows people to adapt to, select, and shape the cultural aspects of their environment"(p. 127). It consists of, and emerges from the interaction of three underlying facets of cultural knowledge, cross-cultural skills, and cultural metacognition.

It is a multifaceted construct drawn from theories of intelligence (Ott \& Michailova, 2015; Sternberg, 1997). But it is distinctive from general intelligence, social intelligence or emotional intelligence (EQ), which are culture bound and are constructed and transmitted within cultures (Earley, 2006; Sharma, 2019). Whereas, CQ is a 'general set of capacities' for diverse cultural environments and applies to more than one culture (Ott \& Michailova, 2015; Sharma, 2019; Thomas et al., 2015). As Earley and Mosakowski (2004) argue, "CQ picks up where EQ leaves off' (p.139).

Thomas et al. (2008) define CQ as a form of intelligence or an individual's ability to effectively interact and also adapt to a foreign culture utilizing one's cultural knowledge, crosscultural skills and cultural metacognition, which then translates into culturally appropriate behaviour. Cultural knowledge involves knowledge about norms, beliefs, values and behaviours appropriate to another culture vis-a'-vis own's culture. Cross-cultural skills include three types of skills; perceptual skills about how perceptions are formed of others and behaviours are interpreted; skills related to how one develops and maintains relationships; and skills related to the ability to adapt and adjust to new cultural situations. Cultural metacognition is the process of regulating and monitoring one's knowledge processes and thoughts in relation to some objectives. It involves awareness of a cultural context, analysis of the influence of the cultural context and planning courses of action appropriate in different cultural contexts (Ott \& Michailova, 2015; Thomas et al., 2008; Thomas et al., 2015).

Studies investigating antecedents of CQ may be categorized under three main subjectsinternational experience and cultural exposure; training/education to develop CQ; and 
individual differences (Ott \& Michailova, 2018). CQ is a type of capability and thus it is open to change and improvement (Wood \& Peters, 2014). The primary factor that can result in higher $\mathrm{CQ}$ is international experience or any other form of cultural exposure. Exposure to another culture through observation and experiencing situations abroad enhances knowledge and awareness about the other culture can also help learn about the culturally appropriate behaviour (Crowne, 2013; Engle \& Crowne, 2014; Wood \& Peters, 2014). However, the type of international experience does matter. Crowne $(2008,2013)$ contends that rather than international vacations, education or work experience abroad leads to higher CQ. Moreover, even short-term international experience can increase CQ (Engle \& Crowne, 2014; Wood \& Peters, 2014).

International experience when combined with cross-cultural education and training can significantly increase CQ (Moon, Choi, \& Jung, 2012; Rehg, Gundlach, \& Grigorian, 2012). Tailored training programmes can improve an individual's knowledge and understanding of another culture and his/her ability to identify cues during international experiences; also equip him/her with the necessary skills to effectively interact across cultures (Macnab et al., 2012; Reichard et al., 2015).

Although CQ capabilities can be acquired, each individual's personal characteristics that are endogenous to an individual, may be related to his/her CQ or acquisition of CQ. Conscientiousness influences metacognitive CQ, agreeableness influences behavioural CQ and extraversion influences cognitive, motivational and behavioural CQ; whereas openness influences all the facets of CQ (Ang et al. 2006; Harrison, 2012). Self-efficacy has been found to be an important trait for acquiring CQ. Cross-cultural experience may be challenging and stressful. Self-efficacy helps an individual endure and persevere these challenges. Also, crosscultural training efforts are more effective when participants are self-efficacious (Macnab \& Worthley, 2012; Macnab et al., 2012).

CQ is found to have a positive relationship with cultural adjustment and performance. Culturally intelligent people are better able to manage their anxiety and uncertainty and adapt to any new surroundings, which helps them focus and channel their efforts more towards work, improving their performance (Malek \& Budhwar, 2013). When exposed to a culturally different environment, people with higher CQ suffer less culture shock (Chen et al., 2011). However, to establish relationships between adjustment and performance, it must be mediated by other enabling factors. Lee and Sukoco (2010) argued that mere expatriate adjustment is not sufficient to improve work performance. Additional programmes to improve expatriates' capabilities related to cross-cultural communication are needed to transform the psychological 
comfort of expats, so that better adjustment lead to better work performance. Compared to the other CQ facets, motivational CQ emerged as more crucial to facilitating adaptation and adjustment (Ott \& Michailova, 2018). A person having greater motivational CQ would be more motivated to gain knowledge and learn about a new culture while expending more effort to overcome issues related to adaptation and adjustment (Huff et al., 2014; Lin, Chen, \& Song, 2012).

Higher CQ helps develop cross-cultural leadership skills. Cross-cultural leaders are required to work in a different cultural environment, with people and processes from multiple cultures (Rockstuhl, Seiler, Ang, Van Dyne, \& Annen, 2011). A higher CQ increases cultural awareness, motivates and helps adapt to a culturally different environment and effectively communicate across cultures, which enhances the cross-cultural leadership effectiveness of an expat (Deng \& Gibson, 2009).

Effects of CQ on cross-cultural communications and relationship building have also been studied. A higher CQ facilitates intercultural coordination and cooperation (Mor, Morris, \& Joh, 2013). Mor et al. (2013) argued that higher cultural metacognition; the process of monitoring and adjusting one's thoughts, makes one aware and conscious of others' cultural preferences during interactions. A higher cultural metacognition helps one to engage in “cultural perspective taking” (Lee, Adair, \& Seo, 2013; Mor et al., 2013) where one considers how another person's cultural background may affect their response to a situation. One can view a situation contextually and from another's perspective. This facilitates more crosscultural coordination and cooperation as it reduces stereotyping and confirmatory bias during interactions. Any ambiguity and uncertainty associated with cross-cultural interactions is reduced as CQ enables one to better predict a culturally different new situation and avoid early judgements (Bucker et al., 2014). This helps develop cross-cultural relationships which enables managers to effectively manage and reduce the ambiguities associated with differences in an institutionally different environment as well (Sharma, 2019).

Individuals high in cultural metacognition can develop affect-based trust enabling creativity in cross-cultural collaboration (Chua et al., 2012). Awareness about another's culture helps one adapt one's behaviour and adjust to cultural differences during interactions which builds interpersonal trust. Thus, trust and effective communication aids creative collaborations. A higher CQ individual is more cooperative, open-minded, agreeable, and has greater interpersonal trust towards culturally different others. Thus, they display cooperative, interestbased behaviours in the context of cross-cultural negotiations which make them effective negotiators (Groves et al., 2015; Imai \& Gelfand, 2010). 
Also, a higher CQ of employees has a positive influence on cross-cultural cross-border knowledge transfers in an MNE (Vlajčić et al., 2019). The different facets of CQ increase cultural knowledge and awareness, help understand others' cultural preferences and intrinsically motivate to engage in cross-cultural interactions that lead to increased knowledge sharing (Chen \& Lin, 2013). Individuals with high CQ are less likely to hide knowledge from culturally different groups as they don't engage in social categorization due to cultural differences (Bogilovic et al., 2017). Therefore, the cultural intelligence of an employee is likely to influence the indirect effect of subsidiary power on knowledge transfers through organizational identification.

\subsection{Summary}

The reviewed literature based on a $2 \times 2$ matrix, involving cross-border knowledge transfer both at the organizational and individual levels, revealed that rich literature exists when both the outcome and antecedents are at the same level. This has resulted in focusing my study on the less explored areas, on how certain identity-based organizational antecedents can influence individual level knowledge transfer within the MNE. This focus area is primarily guided by the knowledge governance approach and the social identity theory. The next chapter proposes relationships of the identified explanatory variables (subsidiary power, intra-MNE competition, and cultural intelligence) on the outcome variable of individual level cross-border knowledge transfer within the MNE. Hypotheses are developed, and a conceptual model is proposed based on the underlying theories and related literature. 


\section{Chapter 3: Hypotheses Development}

\subsection{Introduction}

In the previous chapter, a review of literature was undertaken on cross-border knowledge transfer within the MNE at both the organizational and individual levels. Based on the literature review, I propose my research question on the relationship between subsidiary power and individual cross-border knowledge transfer behaviour. Additionally, two boundary conditions, intra-MNE competition and cultural intelligence are proposed. In the next section, I discuss the underlying theories of KGA and SIT on which the present research is grounded. Following this, I present the conceptual model and hypotheses development. A chapter summary is provided in the last section.

\subsection{Underlying Theories}

Theory is "a set of interrelated abstract propositions about human affairs and the social world that explain their regularities and relationships" (Brewer, 2000, p. 192). According to Sparrowe and Mayer (2011), theory helps building hypothesis in three ways. Firstly, the proposed hypotheses are positioned drawing insights from the related research. Secondly, theory helps with explaining the relationship between the core variables or processes proposed, using clear and logical arguments. Finally, theory helps with presenting the relationships in a coherent manner.

\subsubsection{Knowledge-based view}

Grant (1996), in his seminal article established the "knowledge-based view of the firm". Knowledge, which is valuable, inimitable, and heterogeneous, has been identified as a resource that contributes to the long-term performance of the firm and is a source of its strategic competitive advantage. This view attributes the success of the firm to its superior ability to 
integrate, transfer, and apply knowledge across the firm. Thus the focus of the knowledgebased view $(\mathrm{KBV})$ is on coordination of knowledge within the firm and the primary role of the firm is integration of specialized knowledge. An important assumption of the knowledge-based view is that the locus of knowledge is the individual and the efficiency of knowledge creation and storage depends on individual specialization. The efficiency of the firm in integrating the knowledge embedded in its individual members is determined by the efficiency of the mechanisms used for integration, and the extent to which the knowledge domain matches the requirements of the product (Grant \& Baden-Fuller, 1995).

Kogut and Zander's (1993) influential article on the evolutionary theory of the MNE attributes internalization of the firms and creation of the multinationals to their knowledge that provides them comparative advantage to internalize. According to them, decisions of the firm to transfer technology within the firm depends upon the efficiency of the firm in creating and transforming knowledge into products and services relative to other firms, not on market failure. With this new perspective, intra-organizational knowledge transfer is not only a major activity of the MNE, but also the very reason for its existence. MNEs manage local knowledge, engage in learning international knowledge and at the same time integrate global knowledge (Bartlett \& Ghoshal, 1989). Thus, knowledge drawn from multiple locations and dissemination of this knowledge across the firm becomes the basis of its competitive advantage.

Following the knowledge-based view, it is each individual who is responsible for the creation and storage of knowledge in the organization. This is consistent with Simon's (1991, p. 125) observation that 'all learning takes place inside individual human heads; an organization learns in only two ways: (a) by the learning of its members, or (b) by ingesting new members who have knowledge the organization didn't previously have'. Felin and Hesterly (2007) also argued that any theory on value creation in the organization must start with a consideration of the individuals who make up the organization. Any collective knowledge-based phenomenon can be explained by knowledge at the individual level.

Adopting this idea, the present study explores the integration of valuable knowledge in the multinationals through knowledge transfers by the subsidiary employees. Apart from the formal exchanges initiated by the headquarters or the subsidiary, much of informal exchanges of knowledge and information take place through these individual employees. These informal knowledge exchanges facilitate innovation and influence long term organizational performance. Therefore, this study aims at capturing integration of the knowledge embedded in the individuals across different subsidiaries in the multinational. 


\subsubsection{Social identity theory}

The primary theoretical framework that is used in this study is Social Identity Theory (SIT), which was advocated by Tajfel (1978) and Turner (1975). Social Identity Theory says, people tend to categorise themselves and others into various social groups which are defined by certain prototypical characteristics (Turner, 1975). The Social Identity Theory identifies two components of the concept of "self". One component is personal identity, which encompasses an individual's own attributes such as physical or psychological characteristics, or abilities. The second component is social identity, which involves the attributes of the group he/she is classified under. Thus, social identification is the perception of an individual of oneness of the "self" with the group one belongs to, having typical characteristics. Social identity is synonymous with group identification (Tolman, 1943) and the terms are often used interchangeably. Social identification involves two important processes- a) self-categorization, which perceptually differentiates between in-group and out-group; and b) self-enhancement, that is, in-group favouritism over the out-group (Terry \& Hogg, 1996).

One important form of social/group identification that is pertinent to this study is Organizational Identification (OI), because organizations such as MNEs are entities that can be identified as a social group. "OI is defined as the degree to which a member defines himself/herself by the same attributes he/she believes define the organization" (Dutton, Dukerich, \& Harquail, 1994, p.1). Thus, an individual who is part of an organization like an MNE subsidiary, builds perception about his/her identify based on the characteristics of the organization. Ashforth and Mael (1989) identified a set of antecedents which enhance an individual's identification to a group. These are, group distinctiveness (either positive or negative) with respect to its values and practices, group prestige (in relation to other similar groups), salience of the out-group(s), and inter-group competition, which accentuates the existence of the in-group and enhances identification with the in-group through reinforcement of the differences and similarities with the out-groups.

Terry and Hogg (1996), argue that self-concept based on salient social identity and selfcategorization leads people to construct a context specific group norm, represented by group prototypical characteristics. This group norm describes and prescribes beliefs and behaviours that promote optimum maximization of intergroup differences and minimization of in-group differences. Thus, the individual defines himself/herself and thinks, feels, and behaves in terms of the group norm not only in public (for social approval) but also in private.

Understanding of how group identification can influence peoples' behaviour is informed by various studies in social psychology. The relationship was confirmed by a study on changes 
in individual behaviour in the environmental domain by Rabinovich, Morton, Postmes, and Verplanken (2012). They found that individual intentions and ultimately their behaviour to engage in environmentally friendly actions were influenced by inter-group comparisons and self-categorization. In another study on exercise and sun-protective behaviour, Terry and Hogg (1996) confirmed that perceived group norms of the behaviourally relevant reference group influenced intensions to engage in a particular behaviour when the individual identified highly with the group. Another study by Mael and Ashforth (1992) found that alumni identification with the alma mater predicted individual behaviour of the alumni, where the alumni engaged in financial contributions to the alma mater, and advised offsprings and others to attend the alma mater.

Social Identity Theory is likely to be relevant to this study of knowledge transfer in MNEs as it draws attention to interaction among distinct groups (Child \& Rodrigues, 1996). The different socio-cultural, political, economic, environments that MNE subsidiaries are in, contribute to their distinctiveness and can lead their employees to distinguish them from the parent as well as other subsidiaries. According to Birkinshaw, Holm, Thilenius and Arvidsson (2000), an employee's organizational identification in a foreign subsidiary of an MNE is of crucial importance to the MNE due to the ambiguity and tension that is likely to exist between the foreign subsidiary and the corporate headquarters or between the subsidiaries located in different countries. Empirical support for the idea was found by Gregersen and Black (1992), who reported that managerial employees were affected by separate group identification at the subsidiary and global organizational levels and based on their group identification distinguished between themselves and employees in other subsidiaries/headquarters.

Various researchers have explored the dual organizational identification of subsidiary employees, where the employees have various degrees of identification with both the MNE and the local subsidiary unit (Reade, 2001b; Smale et al., 2015; Vora \& Kostova, 2007). Being part of one group does not involve exclusion from other groups (Reade, 2001a). Employees in an organization may simultaneously belong to different departments, groups, and therefore may identify with various entities at different organizational levels at the same time. It is commonly found that individuals identify more with lower-level entities which are smaller and proximate (Smale et al., 2015), or the work group, than the organization as a whole (Bartels, Pruyn, Jong, \& Joustra, 2007). Therefore, in the MNE, where employees have dual identification with both the subsidiary and the organization, it is likely that managers identify more with the subsidiary than the MNE as subsidiaries are smaller social units closer to them (Reade, 2001a). 
As held by Vora, Kostova, and Roth (2007), subsidiary employees' dual identification with the MNE as well as the subsidiary can be at similar levels or disparate levels, that is, identifying more with one entity than the other. This has implications for subsidiary manager roles and role conflict interactions with the HQ. This study examines knowledge exchange between subsidiaries, where this dual identification with the HQ is unlikely to exert an influence. Regarding employees' decisions on knowledge exchange with members of other subsidiaries, it is their extent of identification with their own subsidiary that will come into play. Work group identification or identification with a smaller group (as the case of the subsidiary) is more important than organizational identification in determining organizational attitude and behaviour (Van Knippenberg \& Van Schie, 2000).

\subsection{Hypothesis Development}

The main outcome variable of interest is interpersonal knowledge transfer-knowledge seeking and knowledge sharing. Interpersonal knowledge transfer refers to exchanges between individuals in an MNE related to the business (product, market, organization etc.). The two main components of interpersonal knowledge transfer are the sender/source, who shares the knowledge, and the receiver, who acquires the knowledge (Liyanage, Elhag, Ballal, \& Li, 2009). Knowledge transfer occurs when individuals actively communicate knowledge/information between them. These exchanges take place between globally dispersed members of project teams or expert groups (Haas \& Cummings, 2015; Morris, Zhong, \& Makhija, 2015; Raab et al., 2014), or individual members, through telephone conversation, emails or other IT mechanisms, or through direct face-to-face contacts at conferences and meetings (Allen, 1977; Bouty, 2000; Hippel, 1987).

Here, for the purpose of this study, the focus is only on knowledge transfers that take place in MNEs involving wholly owned subsidiaries. As we know, due to greater control of the parent on the wholly owned subsidiaries, transfer of knowledge is faster and easier to and from the wholly owned subsidiaries (Buckley, Clegg, \& Tan, 2003; Grosse, 1996; Wang et al., 2004).

While knowledge seeking examines knowledge inflows, knowledge sharing examines knowledge outflows. As proposed, the primary predictor variable is subsidiary power, the mediator is organizational identification, and the moderators are intra-MNE competition and cultural intelligence. The conceptual model is presented in Figure 3.1. 
Figure 3.1 The conceptual model

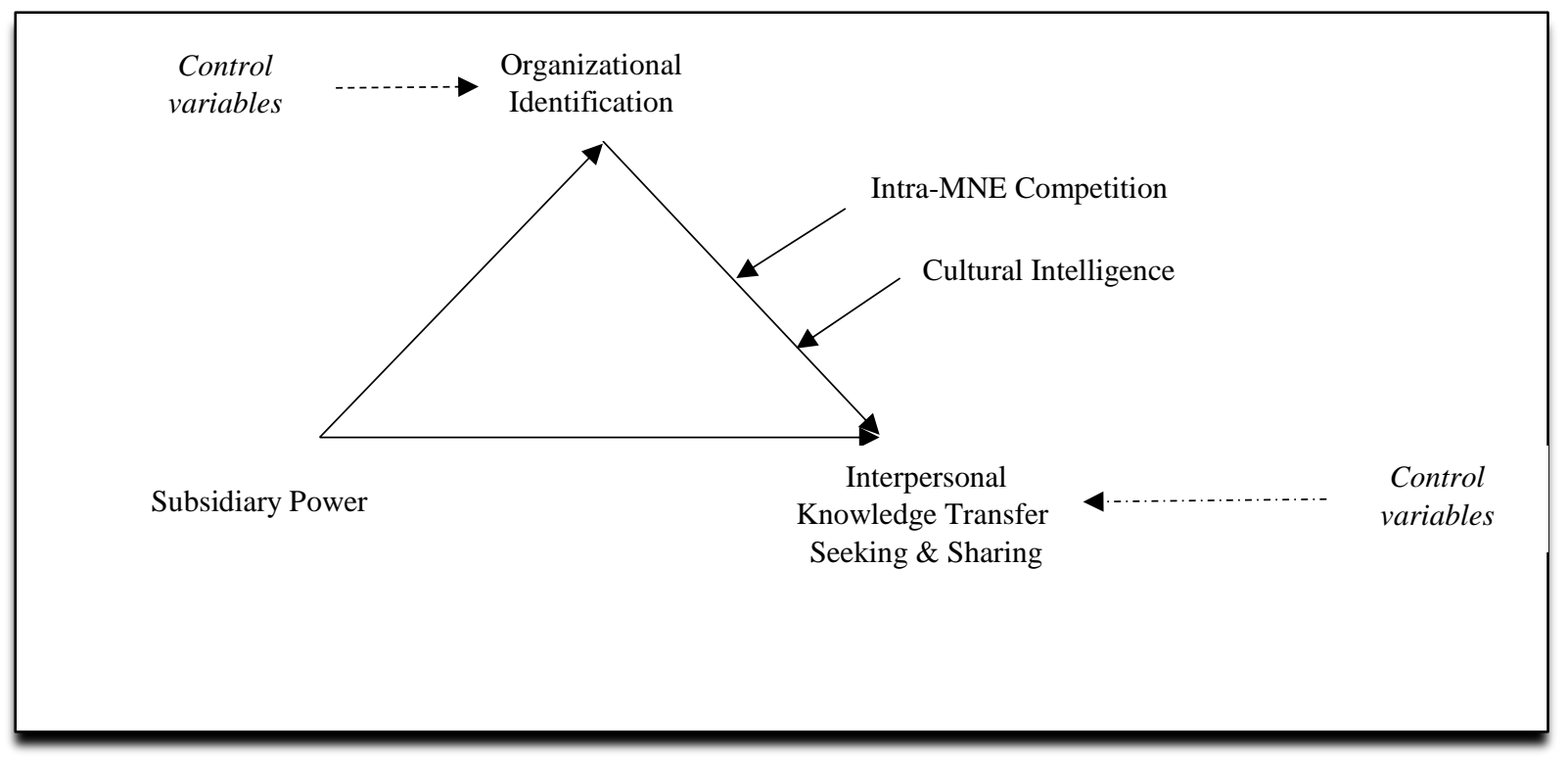

\subsubsection{Direct effect of subsidiary power}

Studies examining the influence of subsidiary power on knowledge transfers within the MNE have primarily dealt with organizational knowledge transfer as the outcome variable. The power of a subsidiary has been found to impact knowledge transfers between subsidiaries when power is based on a resource dependency relationship between them. Wong, Ho, and Lee (2008) posited that when a subsidiary is powerful due to possessing resources upon which another subsidiary depend, there will be more knowledge transfers to the powerful subsidiary. They argued that any request for knowledge by the powerful subsidiary from the low-power subsidiary will always be complied with by the low-power subsidiary due to fear of losing resources that it is needs, which will lead to more knowledge flows. Further discourses as well have focused on organizational level knowledge transfers (Frenkel, 2008; Whittle et al., 2016). However, it can be observed that while these studies drew conclusions about knowledge transfer at the organizational level, their arguments are basically grounded on cross-border interactions of individual actors within the MNE.

In a study by Frenkel (2008) which examined the effect of geopolitical factors on knowledge transfers in multinationals, subsidiary power was drawn from the development status of the host country. Subsidiaries located in a less developed country were considered less powerful and it was found that knowledge transfers were more to the low-power subsidiaries. This was due to employees from high-power units who engage more in knowledge sharing with the low-power subsidiary members. High-power unit employees perceive knowledge of the low-power subsidiaries in less developed countries as inferior to their 
knowledge, due to which they engage in more knowledge transfers to those subsidiaries, sometimes to the extent of even imposing practices on them intervening more sophisticated practices.

Power may deter free flow of knowledge from a low-power unit to a high-power unit. By virtue of power, a high-power unit may have more control and coercive power over the low-power unit. This might create barriers to sharing knowledge and information by the lowpower unit due to fear of the high-power unit. Whittle et al. (2016) found evidence of such behaviour by low-power unit members. They found that based on the resources, capabilities, and knowledge possessed by the subsidiary unit, subsidiary managers make sense of the power they have or the headquarters has over them. This sensemaking guides their reasoning and decision-making. Anticipating adverse reactions from the HQ, they hide important information or knowledge of the local market from the headquarters which the authors term sensecensoring. This has implications for flow of intra-MNE knowledge as knowledge flow from the low-power subsidiary to the high-power HQ will decrease. Thus, while knowledge inflows to a low-power subsidiary will be more, outflows will be less.

Some studies have highlighted the existence of micro-political behaviour among individual actors in cross-border interactions. Dörrenbächer and Geppert (2011) contend that dynamics of power in a MNE impact subsidiary managers' socio-political interactions, like knowledge sharing, with peer subsidiary managers through which they try to exert and develop power for themselves or their subsidiary. The main focus in these studies was on how key actors in the subsidiary pursuing different interests developed political-strategies to initiate, influence, or negotiate various processes with the HQ which benefited the subsidiary (Geppert \& Dörrenbächer, 2014).

Conroy, Collings, and Clancy (2018) fortified this view by positing that subsidiary actors use their social and political skills in micro-level interactions to enhance their subsidiary influence in MNE strategic decision making. These interactions are mechanisms to building relationships and coalitions with key decision makers. Through these interactions they try to showcase or reinforce certain valuable subsidiary characteristics to make the HQ aware and draw its attention. This involves proactive behaviours such as identifying key decision makers, seeking information, feedback seeking, or network building, which leads to more knowledge inflows from the high-power.

Dependency relationships also give rise to power in MNEs (Mudambi et al., 2014). By seeking help, the seeker acknowledges his/her dependence on the helper/advisor (Druian \& DePaulo, 1977). The seeker's dependence on the advisor makes the advisor relatively more 
powerful than the seeker, and by acknowledging incompetence and dependence the seeker puts him/herself at a relatively power-less position than the advisor. This help-seeking behaviour has implications for power (Lee, 1997), which makes a subsidiary vulnerable to losing power in the MNE. Therefore, members of a high-power subsidiary are less likely to seek knowledge from a low-power subsidiary and low-power subsidiary members are more likely to seek knowledge from a high-power subsidiary.

Moreover, expertise and value of knowledge held by a source is an important consideration for seeking knowledge from the source (Borgatti \& Cross, 2003). Knowledge may be a source of power for subsidiaries (Mudambi \& Navara, 2004). Knowledge held by a powerful subsidiary may be perceived as more valuable than that held by a less powerful unit. This encourages more knowledge seeking from the high-power subsidiary than the low-power one.

Based on the above arguments, I posit that the relationship between power of a subsidiary and knowledge seeking is likely to be negative, but with knowledge sharing it is positive. Therefore, I hypothesize that

Hla: Subsidiary power has a negative direct effect on interpersonal knowledge seeking. H1b: Subsidiary power has a positive direct effect on interpersonal knowledge sharing.

\subsubsection{The mediating role of organizational identification}

To argue for the mediating effects of OI in the relationship between subsidiary power and knowledge transfer, three relationships are argued. The first is the direct effect of subsidiary power on knowledge transfer which is argued in hypothesis 1. Next I will argue for the effects of (i) subsidiary power on organizational identification, and (ii) organizational identification on knowledge transfer, to make a case for the mediation hypothesis.

The power of a subsidiary plays a crucial role in a subsidiary's development as well as its survival in the MNE. A subsidiary's evolution through role development or charter change depends on how much influence or power the subsidiary has over HQ's decision (Balogun et al., 2011; Birkinshaw \& Hood, 1998; Dörrenbächer \& Gammelgaard, 2006). Power, as a distinctive characteristic of the subsidiary, distinguishes it from other subsidiaries and the HQ in the MNE.

Various researchers have found effects of power on self-categorization (Smith, Smallman, \& Rucker, 2016), and inter-group relations (Hornsey, Spears, Cremers, \& Hogg, 2003; Sachdev \& Bourhis, 1991). The more attractive the construed external image or the perceived external prestige is, as with a more powerful subsidiary, the greater will be the 
organizational identification of the employees (Bartels et al., 2007; Dutton et al., 1994; Smidts, Pruyn, \& Van Riel, 2001). Power contributes to the distinctiveness and prestige of the subsidiary in the multinational which confers an employee affiliated to that subsidiary with enhanced organizational identification in his/her interactions with the out-groups or other subsidiaries/parent.

Relative power of the subsidiary can thus become a key driver of organizational identification in the MNE and lead to social categorization and stereotyping within the MNE (Hornsey et al., 2003). In a similar premise about influence of power, Fiol et al. (2001) put forward the concept of "Power Mental Models". According to them, power affects generation of individual perceptions or mental models about the power of their own subsidiary as well as other subsidiaries (Fiol et al., 2001). These mental models or a set of beliefs are generated based on cues such as organizational position, control of resources and other power sources. Thus, higher subsidiary power leads to higher organizational identification.

Organizational identification leads employees to engage in categorizing and stereotyping in the MNE and promotes behaviour that is consistent with their concept of power (Fiol et al., 2001). Self-categorization and inter-group comparisons influence individual behaviour, where behaviour is regulated by the perceived norms and standards associated with the salient group membership (Rabinovich et al., 2012). Inter-group comparisons are made to bring-out the salience of the in-group, as social categories become salient only with respect to other relevant categories (Tajfel \& Turner, 1979). Terry and Hogg (1996) argue that self-concept based on salient social identity and self-categorization, leads people to construct a context-specific group norm, represented by group prototypical characteristics. This group norm describes and prescribes beliefs and behaviours that promote optimum maximization of intergroup differences and minimization of in-group differences. Thus, the individual defines himself/herself and thinks, feels, and behaves in terms of the group norm not only in public (for social approval), but also in private. Thus, when individuals identify with the organization, they will display behaviour that is conducive to the organization that enhances its performance (Ashforth, Harrison, \& Corley, 2008; Bartels et al., 2007; Reade, 2001a).

Through self-categorization based on attributes or bases of power, individuals try to verify and maintain their self-conceptions about power of theirs as well as other subsidiaries, and engage in behaviour that is consistent with this self-conception (Fiol et al., 2001). They strive to promote optimum maximization of this inter-subsidiary difference of power. If they belong to a high-power subsidiary, they would try to protect and maintain their high power, whereas, low-power subsidiary members will constantly try to enhance and develop their 
power. Doing so, they would strive to hold on to their unique resources and capabilities which can confer them power.

Thus, subsidiary power due to organizational identification of an employee will influence individual knowledge transfer/exchange behaviour with members of other subsidiaries in the MNE. Knowledge, a resource, is of strategic importance to the subsidiary as it is a source of a subsidiary's competitive advantage (Argote \& Ingram, 2000), and possession of knowledge and capabilities is a source of power to the subsidiary within the MNE (Mudambi \& Navarra, 2004). If they belong to a relatively powerful subsidiary, they would not only protect themselves from losing the resources that confer them power, but also refrain from engaging in any kind of behaviour which may question their power status. They would avoid such behaviour that may create a gap or discrepancy between the desired and perceived image about their power, or create a perceived/construed image that is inconsistent with the image of a powerful subsidiary. This is similar to what Roberts (2005) asserted about employing defensive social identity-based impression management strategies to protect or maintain one's image as powerful and avoiding situations that puts one at a powerless position.

Roberts (2005) incorporated social identity theory to professional impression management in organizations and termed it social identity-based impression management. She posited that social identities shape an individual's desired and perceived professional imagehow he/she wishes to be seen and how one thinks he/she is currently seen, as a member of a social identity group. One wants to be either affiliated with or distanced from the stereotypical or idiosyncratic characteristics of the social identity group/groups one belongs to. He/she responds to identity threats and tries to shape other's perceptions to bridge the gap between desired and perceived professional image. Thus, he/she engages in professional image construction to construct the image he/she values, through impression management strategies which are employed during interpersonal interactions. These strategies may be both assertive and defensive strategies. Assertive strategies are used to create the desirable image, whereas, defensive strategies are used to maintain a particular image.

Thus, I establish how subsidiary power can influence cross-border individual knowledge transfer behaviour in an MNE using organizational identification or the Social Identity Theory. The underlying theory used to explain the relationship is represented by the mediation effect of organizational identification on which the relationship is grounded. Thus, I propose that 
H2a: Organizational identification of employees would mediate the influence of subsidiary power on interpersonal knowledge seeking.

H2b: Organizational identification of employees would mediate the influence of subsidiary power on interpersonal knowledge sharing.

\subsubsection{The nature of indirect effects}

After hypothesizing that organizational identification is the mechanism through which subsidiary power influences interpersonal knowledge transfer, next I argue about the direction of such effects.

One of the primary processes in SIT involves self-enhancement (Terry \& Hogg, 1996). Self-enhancement is the desire to see oneself or one's actions, attitudes, traits in a positive light (Pfeffer \& Fong, 2005). Individuals seek knowledge from higher power subsidiary members for self-enhancement. Pfeffer and Fong (2005) in their study on self-enhancement and its relation to power processes, posit that people prefer to associate themselves with winners, with successful people or more powerful ones, to feel positive about themselves and enhance their self-image. This is due to their desire to "bask in reflected glory", which Cialdini et al., (1976) demonstrated in their classic study where a higher number of football fans were found wearing university-insignia apparels after the university football team won. Through association and building relationships with the more powerful, individuals enhance their status (Pfeffer, \& Fong, 2005). A similar effect was observed in a study on information flow in R\&D laboratories (Allen \& Cohen, 1969). They found that PhD scientists rarely socialized and discussed technical problems with the non-PhDs. In contrast, non-PhDs directed most of their socialization and technical discussion to $\mathrm{PhDs}$ rather than to non-PhDs. In laboratories, recognition, publication and other forms of status representations are mostly restricted to the PhDs. Therefore, through association with the higher status PhDs, the non-PhDs attempt to enhance their status.

Similar to self-enhancement motive, is "opportunity focused personal identification", proposed by Ashforth, Schinoff, and Rogers (2016). According to this approach, an individual tries to identify with another person if he/she sees a chance to uphold his/her current valued identity or enhance or acquire new valued identity. Identifying and building relationships with members of a more powerful subsidiary is likely to enhance individual power and status. While individuals will choose to seek knowledge from a high-power subsidiary member, they will avoid seeking from a low-power one. It is perceived that associating with the unsuccessful or 
the negative demeans an individual's image and status. So they choose not to associate with and seek knowledge from the less powerful subsidiary members.

In addition to the motive of status enhancement, Social Identity Theory also incorporates status protection. Group members who already have a positive social identity will strive to maintain the status quo (Tajfel \& Turner, 1979). Member of a subsidiary having high power will tend to maintain the powerful image by not seeking knowledge from a low-power subsidiary. By seeking help, help seekers acknowledge that they are incompetent, or there is a gap in their knowledge and expertise (Ames \& Lau, 1982; Karabenick \& Knapp, 1988).

Showing off their knowledge gap or incompetency will infuse them with fear of losing power, as knowledge and expertise are one of the primary sources of power (French \& Raven, 1968; Lee, 1997; Salancik \& Pfeffer, 1977). This counters their need for self-enhancement, as it is detrimental to the knowledge seeker's need for creating a positive public impression about himself/herself (Leary \& Kowalski, 1990; Lee, 1997; Schlenker \& Weigold, 1992) and maintenance of positive self-image (Cialdini \& Nicholas, 1989). Whereas, seeking knowledge from the powerful is favourable for self-enhancement, as individuals are willing to subjugate their interests and emotions in order to associate with the powerful (Pfeffer \& Fong, 2005). Thus, knowledge seeking will be high by a low-power and knowledge sharing will be high by a high-power (due to seeking by the low-power).

Also, knowledge sharing provides an opportunity to the knowledge holder to show-off his/her knowledge and capabilities and differentiate him/herself from others, which can bring power and status. Need for status can drive sharing of knowledge and expertise (Park et al., 2017). This would lead to lesser inflow and more outflow of interpersonal knowledge to and from a high-power unit due to the unit members engaging in less knowledge seeking and more knowledge sharing.

Therefore, it can be hypothesized that,

H3a: Subsidiary power will have a negative indirect effect on interpersonal knowledge seeking mediated through organizational identification.

H3b: Subsidiary power will have a positive indirect effect on interpersonal knowledge sharing mediated through organizational identification.

\subsubsection{Moderating effect of intra-MNE competition}

Intra-MNE competition moderates the indirect effect of subsidiary power on interpersonal knowledge transfer through organizational identification of employees. In the MNE, when subsidiaries are in competition, they look for ways to strengthen their bargaining power and 
position within the organization at the expense of their competitor subsidiaries, and behave opportunistically (Dörrenbächer \& Gammelgaard, 2016). Moreover, the competitor might hide his/her knowledge and declare only parts of the related knowledge so that the seeker is only benefited partially. This increases the seeker's search cost as he/she needs to spend additional effort searching to obtain the useful knowledge (Hansen, Mors, \& Lovas, 2005). Therefore, there is relatively less trust on the knowledge shared by the competitor rather than a noncompetitor.

Szulanski (1996) has identified that one of the reasons for stickiness of knowledge is the characteristics of the knowledge source. According to him, perception of the source as unreliable and untrustworthy leads to resistance and challenge from the recipient during transfer and makes the knowledge sticky. Lack of trust in the competitor subsidiary also has a negative influence on sharing of knowledge with the members of the competitor subsidiary. Trust here implies the degree to which one believes that the other individual has good intentions and does good out of altruistic motives towards him/her (He, Fang, \& Wei, 2009). Thus, trust not only creates barriers in knowledge seeking, but also in knowledge sharing (Chen \& Hung, 2010; Chiu, Hsu, \& Wang, 2006; Lai, Chen, \& Chang, 2014; Toh \& Srinivas, 2012).

Decisions to share knowledge with others are based on the expectation of reciprocity, that is, expectation of future benefits from the recipient of the knowledge. An expectation of reciprocity is an essential element of exchange of resources (Gouldner, 1960). Expectation of reciprocity is influenced by the presence of inter-firm competition (Hippel, 1987; Schrader, 1991). When the request for knowledge is from a competitor, the expectation of reciprocity of the source from the recipient is likely to be less, as by spending time and effort in sharing the know-how, the source may benefit the recipient and itself lose its ability to compete. In a study, Kachra and White (2008) found that R\&D scientists who belong to different project groups, competing for limited seed money, investment dollars, and budget allocations within the organization, made smaller efforts to fulfil requests for know-how from scientists in other groups due to intergroup competition.

In addition to expectations of reciprocity, knowledge sharing decisions are also based on anticipated costs and benefits from the sharing activity (Appleyard, 1996). It depends on whether the expected benefits from giving away the knowledge are more than the expected costs. In the MNE, when a subsidiary member shares his/her knowledge with a competitor subsidiary member, he/she risks the loss of the subsidiary's unique knowledge that could provide the subsidiary with competitive advantage over its competitor subsidiaries and increase its bargaining power within the MNE. Possession of unique knowledge confers monopoly 
rights to a firm, and by sharing that knowledge with the competitor, the firm gives up the monopoly over the knowledge and faces "competitive backlash" (Carter, 1989, p.156; Schrader, 1991). Thus, the cost of sharing outweighs the benefits due to sharing. Absence of competition between the exchange partners is an important consideration for sharing knowledge (Bouty, 2000).

Another factor that inhibits knowledge seeking behaviour is, acknowledgement of incompetency or knowledge gap in front of the source (Ames \& Lau, 1982; Karabenick \& Knapp, 1988) and dependence on the source. Through seeking knowledge, seekers display their knowledge gap or incompetency. As knowledge and expertise are sources of power (French \& Raven, 1968; Salancik \& Pfeffer, 1977), displaying knowledge gap will make them vulnerable to losing power in intra-MNE bargaining. This is also harmful to the seeker's need for selfenhancement, creation and maintenance of his/her positive self-image (Cialdini \& Nicholas, 1989; Leary \& Kowalski, 1990; Schlenker \& Weigold, 1992). Also, it is believed that, by seeking help, the seeker acknowledges his/her dependence on the source and confers more power to it (Druian \& DePaulo, 1977). Based on the resource dependency theory, Emerson (1962) posits that a dependency relationship makes an actor on whom others are dependent, more powerful. Therefore, seeking help from a member of a subsidiary which is a strong competitor as well, will bestow more power to it.

The above arguments lead me to posit that intra-MNE competition creates barriers to both knowledge seeking and sharing, the indirect effect of subsidiary power on interpersonal knowledge transfer is likely to depend on the extent of competition between the subsidiaries. Thus, it can be hypothesized that:

H4a: Intra-MNE competition strengthens the negative indirect relationship between subsidiary power and interpersonal knowledge seeking.

H4b: Intra-MNE competition weakens the positive indirect relationship between subsidiary power and interpersonal knowledge sharing.

\subsubsection{Moderating effect of cultural intelligence}

Cultural skills are very important for individuals who engage in knowledge transfers or any inter-unit interactions in an MNE (Barner-Rasmussen et al. 2014). Employees who have higher CQ are more motivated to interact with culturally different others. They have greater understanding and respect for the culture and values of others, while the ones with low CQ engage in stereotyping (Kim and Dyne, 2012). As put by Ang and Dyne (2008), their understanding of key cultural differences makes them overcome any prejudices to collaborate 
with others from different cultures. They are better able to discover international opportunities and be innovative (Lorenz et al., 2018). Cultural knowledge enhances creativity of individuals as they draw culture-related novel ideas from their culturally diverse networks (Chua, 2015).

This capacity to identify and the ability to tap opportunities in a multicultural environment helps high CQ employees transcend organizational boundaries created by their subsidiary identification and power. They have intrinsic willingness to learn from crosscultural challenges. They are less risk averse, initiative taking, and promote cooperation that have a positive impact on innovation in a multi-cultural context (Elenkov \& Manev, 2009). Their capability to initiate efforts to source and integrate resources helps them overcome the inhibitions to engage in cross-border knowledge seeking from culturally different MNE members.

Cultural differences give rise to social categorization where individuals engage in ingroup out-group distinction with culturally different MNE members. Higher CQ is positively related to sharing knowledge in multi-cultural teams (Chen \& Lin, 2013). High CQ individuals are less likely to engage in culture induced social categorization and knowledge hiding (Bogilović et al., 2017). They understand diverse cultural backgrounds and values of culturally different people well and are able to engage in "cultural perspective taking" (Lee et al., 2013; Mor et al., 2013). 'Cultural perspective taking' is the ability to understand how a person's cultural background may influence his/her response to a particular situation. This helps develop shared values and understanding (Groves \& Feyerherm, 2011) and facilitates coordination and cooperation among culturally diverse MNE members who endorse similar guiding principles (Adair, Hideg, \& Spence, 2013).

High CQ improves cross-cultural interpersonal communication which is indispensable for any type of creative collaboration (Chua et al., 2012). The different facets of CQ directly and indirectly influence knowledge sharing behaviour (Ang et al., 2007). With cognitive CQ, individuals have knowledge, awareness of others' cultures, also about similarities and differences with their own culture. Metacognitive CQ helps them acquire, understand cultural knowledge, others' cultural preferences, and accurately interpret behaviours during interactions. High metacognitive and cognitive CQ also enables high CQ individuals to use their prior culture-specific knowledge and acumen to identify profitable opportunities that exist in a multicultural environment (Lorenz et al., 2018). Cultural metacognition is crucial for individuals' creativity in the global context (Chua \& Ng, 2017). Although cultural knowledge is positively related to creativity of individuals (Chua, 2015), higher cultural knowledge may be detrimental to creativity. But, it was found that cultural metacognition can mitigate this 
negative effect as this effect is more prominent on individuals who are low in cultural metacognition (Chua \& Ng, 2017). Motivational CQ directs attention and effort towards learning through interaction with culturally different others. This leads one to look for opportunities to interact with out-group members (Bogilović et al., 2017). Finally, behavioural CQ enables one to exhibit culturally appropriate behaviours while interacting with culturally different people. Due to better understanding and ability to interpret messages well during communication, interactions and information sharing becomes more efficient and effective. Thus, the inhibition to share knowledge with a culturally different group member will decline and knowledge hiding will reduce (Bogilović et al., 2017).

A higher CQ enhances both knowledge seeking and sharing with culturally different others. A high-power subsidiary member with high CQ will be motivated to acquire knowledge from another subsidiary member when he/she identifies a potential knowledge source. The negative effect of subsidiary power on knowledge seeking will be mitigated as a high CQ individual has a cooperative mindset that motivates him/her to interact with people from different cultures (Imai \& Gelfand, 2010). Also, the positive effect of power on knowledge sharing will be strengthened as a high CQ individual is able to overcome any inhibition that may lead him/her to share less or hide knowledge from members outside the subsidiary. These high CQ individuals are open-minded, curious, and they enjoy learning new things and interactions with people from different cultures. So, they are less likely to maintain strong ingroup out-group distinctions based on subsidiary power or their identification with the subsidiary. Thus, while the negative effect of subsidiary power on seeking will be weakened, the positive effect on sharing will be strengthened by higher CQ.

Therefore I hypothesize that,

H5a: Cultural intelligence weakens the negative indirect relationship between subsidiary power and interpersonal knowledge seeking.

H5b: Cultural intelligence strengthens the positive indirect relationship between subsidiary power and interpersonal knowledge sharing.

The proposed conceptual model controls for the relevant control variables which are discussed in section 4.3.3.4 in the chapter: Research Design.

\subsection{Summary}

In this chapter, I have explained and argued for the relationships between the constructs that I proposed, using underlying theories. The conceptual model shows that subsidiary power contributes positively to knowledge sharing and negatively to knowledge seeking. Then the 
mediating role of organizational identification and moderating roles of intra-MNE competition and cultural intelligence are presented. Identification mediates the relationship between subsidiary power and knowledge transfer, and the indirect effect is negative for knowledge seeking and positive for knowledge sharing. Competition strengthens and cultural intelligence weakens the indirect negative relationship with seeking. Whereas, competition weakens and cultural intelligence strengthens the positive indirect effect with sharing. In the next chapter, I present a discussion on the design of my proposed research, and on the methodology that is adopted to undertake the study. 


\section{Chapter 4: Research Design}

\subsection{Introduction}

This chapter begins with a discussion of the research paradigm that guides this research. Later, I describe the methodology that is adopted to conduct this study which includes discussion on sample selection, data collection procedures, measurement of variables and statistical techniques used for data analysis.

\subsection{Research Paradigm}

The research paradigm is the fundamental belief or philosophical dimension that guides a research. It is a set of assumptions and beliefs about how we perceive the world that becomes our thinking framework and influences our behaviour as a researcher (Jonker \& Pennink, 2010). As Crotty (1998) defines, theoretical perspective is "the way of looking at the world and making sense of it. It is how we know what we know". The two main philosophical dimensions are ontology and epistemology (Saunders, Lewis, \& Thornhill, 2009; Lincoln \& Guba, 2000). Ontology can be understood as "the nature of existence" while Epistemology as "the nature of knowing" (Berry et al., 2011, p. 283). Ontology is the belief about the nature of knowledge and epistemology is the belief on how this knowledge can be generated, understood and used for it to be acceptable (Wahyuni, 2012).

What strategies a researcher undertakes to conduct his/her inquiry to acquire knowledge is guided by both his ontological and epistemological beliefs (Guba \& Lincoln, 1994). Based on these philosophies, four research paradigms are postulated; Positivism, Postpositivism, Interpretivism and Pragmatism (Guba \& Lincoln, 2005; Saunders et al., 2009, p.119; Wahyuni, 2012). Positivism and post-positivism share a common view that social reality is objective and external and can be measured and understood objectively with quantitative methods. 
Interpretivism believes that reality is constructed by social actors by their perception and interpretation of the social phenomena. This involves subjective analysis and rich description of qualitative data. Pragmatism on the other hand, instead of believing in either a subjective or objective perspective, takes a mixed approach where the research question determines the framework. It favours both quantitative and qualitative data.

\subsubsection{Post positivism}

Although I undertake an objective approach, I do not completely conform to positivism. Positivism perceives the world as highly systematic and uniform, and believes that knowledge is objective, and we can discover this knowledge which is accurate and certain (Crotty, 1998). I would like to follow a post-positivist approach which challenges the belief of the 'absolute truth'. Although post-positivism follows the positivist thinking, it challenges the precision, certainty and absolute truth of knowledge as positivism (Phillips \& Burbules, 2000). It suggests probability instead of certainty. It assumes that reality exists, but also acknowledges the fact that this reality can only be studied imperfectly (Mertens, 2004). It believes that with respect to studying human behaviour, knowledge is the result of social conditioning. Therefore, social reality needs to be framed in a context of dynamic social structures that have created the observable phenomena within social world (Wahyuni, 2012).

My study aims to investigate human behaviour in an organizational context which is embedded in a socio-cultural dynamic environment. For this purpose, I have reduced the ideas into variables comprising hypotheses that are testable. The objective of my research is to study the effect of subsidiary power, employees' cultural intelligence and intra-MNE competition on interpersonal cross-border knowledge exchanges. I believe that, "the reality" or "what we want to know" exists regardless of the "operation of any consciousness"; they are objectified in the subjects and we can discover that objective truth (Crotty, 1998, p. 8). So, I investigate the relationships with the help of statistical tests involving testing of hypotheses. I believe that the objective reality about the individual knowledge exchange behaviour and its causes exists, and are measurable with quantitative data; and I am able to discover and assess this knowledge accurately to a certain extent. Therefore, the research paradigm that informs my study is postpositivism.

\subsubsection{Quantitative approach}

The importance of research methodology and the fit between the theory and the methods, has been highlighted by various scholars (Bono \& McNamara, 2011; Edmondson \& Mcmanus, 2007; Maanen, SØrensen, \& Mitchell, 2007; Scandura \& Williams, 2000). The methodology 
or the research design process is the backbone of a study which strengthens the reliability of the study by impacting the reader's confidence on the conclusions drawn by testing the researcher's arguments (Bono \& McNamara, 2011). Edmondson and Mcmanus (2007), suggested a framework (Figure 4.1) for achieving methodological fit which recommends matching the three methodological approaches-quantitative, qualitative and hybrid, to the three levels of prior work or stages of theory development in the field. For a theory at a nascent stage, a qualitative study is recommended, one at a mature stage requires a quantitative study and finally, the one at an intermediate stage should use both qualitative and quantitative.

Figure 4.1 Methodological fit

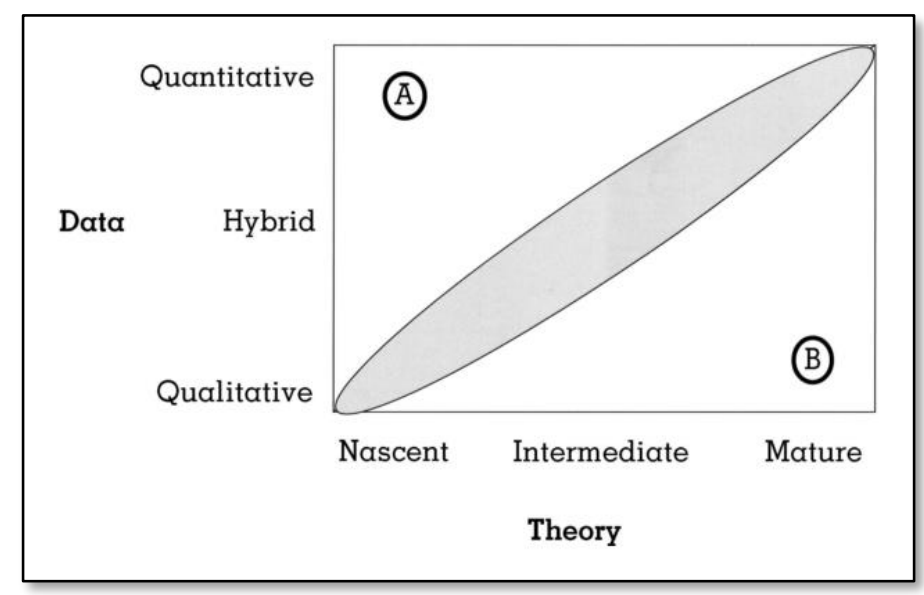

Source: Edmondson \& McManus, 2007, p.1168

The present study is based on theories such as the Knowledge Based View and the Social Identity Theory, which have been researched extensively in various settings. This study intends to bring more refinement to these interrelated theories. The enquiry also involves development of testable hypotheses to establish relationships between constructs (variables) such as subsidiary power, interpersonal intra-MNE knowledge exchanges, intra-MNE competition and cultural intelligence. These are well established constructs which have been used in many studies across different disciplines to explain various phenomena. Interpersonal knowledge exchange is a well-researched topic primarily in human resource management, organizational behaviour, psychology, IT and to some extent in international business as well.

Antecedents of cross-border interpersonal knowledge exchange ranges from social ties, trust, individual characteristics, national and demographic differences (Haas \& Cummings, 2015; Ma"kelä et al., 2007; Raab et al., 2014; Tortoriello et al., '2012). This is a mature field of study. With focused research questions, I attempt to elaborate and extend our understanding of the theories in relation to the topic of interest. Therefore, I believe that a quantitative research 
approach befits studies like this, which are based on "Mature Theories" (Edmondson \& Mcmanus, 2007). A quantitative approach also enables us to study multiple relationships among multiple constructs by examining a large number of observations (Ang, 2014). Moreover, a postpositivist objective philosophy underlying this research espouses the use of quantitative data to bring precision and accuracy to the measurements of the constructs and hypotheses testing.

\subsection{Data Collection Method}

I collected data using a questionnaire survey. A survey is used to capture and establish the views and perceptions of the people about what they feel, believe or value, that is then used to support an argument. It is conducted on potential respondents sampled out of a population so that conclusions can be generalised widely (Jankowicz, 2000). A questionnaire consists of a set of questions which is used to generate data to meet specific research objectives (Clow \& James, 2014).

\subsubsection{Survey design}

A questionnaire was designed (see Appendix 1) with an objective to capture data regarding all the different variables used in the study including control and marker variables. The questionnaire consists of 65 questions encompassing questions on a range of subjects related to respondent demographics, attitudes, behaviours, knowledge, intentions, propositions, and predispositions (Ambrose \& Anstey, 2010). Two different types of questionnaires were designed-one paper based and the other online using the popular user-friendly tool, the Qualtrics.

The questionnaire is primarily divided into four sections excluding the introduction and respondent contact information, which is kept optional. Section A consists of questions related to knowledge inflows. It includes questions about the source of knowledge; the contact person, location of the contact subsidiary, about the knowledge received, task interdependence with the contact, power of the contact subsidiary, competition with the subsidiary and relationship with the contact. Likewise, Section B consists of similar questions related to knowledge outflows; about the individual recipient of knowledge and his/her subsidiary. Section C assesses individuals' identification with their organization, their cultural intelligence and fashion consciousness (marker variable). Section D includes demographic questions about the respondent. 
The language and wording of the questions have been kept simple and easy to understand. Special care has been taken to ensure language comprehensibility and keep the questionnaire layout clean and user-friendly. The questionnaire was prepared only in English. English is one of the two languages used for official purposes in India, the other being Hindi. English is widely spoken and also primarily used for communication in the corporate sector. Additionally, considering the profile of the target respondents, it is highly unlikely that they would not be able to understand and respond to the questionnaires in English.

Dichotomous, open-ended and rating scales are used for the questions. All the interval scale questions use 5-point Likert scale items. A desirable scale for Likert scale question ranges from five-to-eight point response options (Lietz, 2010). A five-point scale was chosen. Having more points may reduce the response rate and response quality due to more points. The respondent takes longer to respond which may lead to withdrawal from the survey (Sachdev \& Verma, 2004). A five point scale is also used following a measure of 'subsidiary autonomy' used in Ambos et al. (2010). The respondents were assured full confidentiality of their data. If they wished, they could also keep the information they provided anonymous. The section of the questionnaire which could identify the respondent was kept optional.

\subsubsection{Pre-test and ethics}

To determine the clarity and comprehensibility of the questionnaire, the questionnaire was pretested using a total of eight faculty and doctoral students in the university (DeVellis, 2017; Krishnan, Martin, \& Noorderhaven, 2006). Pre-testing helped assess the wording, content, difficulty level, and scale of the questions (Lietz, 2010). The face validity of the constructs were also assessed. Based on the feedback, the questionnaire was revised and refined. As the study would require human interaction, approval of the university ethics committee was sought before starting the data collection. The study was granted Victoria University of Wellington Human Ethics Committee approval with reference number: 0000024345.

\subsubsection{Measurement instruments}

\subsubsection{Dependent variable-interpersonal cross border knowledge transfer}

To collect information on interpersonal knowledge exchange, the questionnaire was addressed to individual respondents in the subsidiary, the bench-level scientists, which are the scientists and engineers. Knowledge exchanges among the scientists in the R\&Ds may not be confined to a specific area of their research (Kachra \& White, 2008). These exchanges also involve any kind of work-related information and knowledge sharing which is common to most of them regardless of any specific research area. This study aims to capture any type of job-related 
"know-what" and "know-how" (Kogut \& Zander, 1992) exchanges between the employees. Here an egocentric approach was applied to find out whom the individual employee contacts for work related information, know-how, advice, and who contacts him/her in reverse (Cross \& Cummings, 2004; Nebus, 2006). Interpersonal knowledge exchange involves two activities: knowledge seeking and knowledge sharing (Reinholt et al., 2011). Therefore, both the activities of seeking and sharing are examined separately to understand individual knowledge inflows/outflows or transfers to and from the focal subsidiary.

The first objective was to identify the particular contact from whom the individual receives knowledge and the one with whom he/she shares knowledge. Therefore, the name generator technique was used following Cross and Cummings (2004); Mäkelä and Brewster (2009); and Reagans and McEvily (2003). Sections A and B begin with a name generator question asking respondents to identify the contact with whom the respondent had engaged in information and know-how exchange, seeking and sharing respectively, during the previous 12 months through email, telephone, video conferencing, or face-to-face meetings. Once the respondent identifies the contacts, the rest of the questions in each section are with reference to the particular contact.

Knowledge exchange was measured by the amount and utility of knowledge that the respondent received and shared through seeking and sharing respectively. Following Hansen (2002), Mäkelä and Brewster (2009), and Kogut and Zander (1992) both 'amount' and 'use' of knowledge were measured using four items each. Knowledge is captured by the items which include 'facts or information', 'advice', 'personal insights' and 'tricks of the trade'. A fivepoint Likert scale ' $1=$ Very little to $5=$ Very much' was used to measure the 'amount' of knowledge and a scale of ' $1=$ Not at all useful to $5=$ Extremely useful' to measure 'usefulness' of the knowledge received or shared with the contact.

For knowledge seeking, the amount of knowledge received is measured by "During the last 12 months I have received for the identified contact ( $1=$ very little, $2=$ little, $3=$ neither little nor much, 4=much, 5=very much) 'Facts or information', 'Advice to deal with work-related problem', 'Personal insights about work' and 'Tricks of the trade'. Usefulness of knowledge received is measured by "Please rate the usefulness of knowledge/information you have received from this contact (1=not at all useful, 2=slightly useful, 3=useful, 4=very useful, $5=$ extremely useful). I find, 'Facts or information', 'Advice to deal with work-related problem', 'Personal insights about work' and 'Tricks of the trade'. Knowledge sharing is measured with similar questions. After conducting confirmatory factor analysis, values for knowledge seeking and knowledge sharing were imputed using AMOS version 23. 
As the objective of this study is to capture any type of knowledge exchange that an individual employee engages in, a subjective measure of knowledge transfer is more appropriate than an objective measure such as patents or citations. Patents and citations are the tangible or visible outcomes of innumerable knowledge transfer processes that occur behind the scene. Therefore, an objective measure is likely to present a partial picture only.

\subsubsection{The main explanatory variable}

The research investigates a causal relationship between subsidiary power and interpersonal knowledge exchanges-seeking and sharing. Following Najafi-Tavani et al. (2015) and Ambos et al. (2010), power of a subsidiary was defined by its influence over HQ's decisions and its autonomy. In these studies, influence and autonomy of the subsidiary are measured by the perception of the subsidiary manager/general manager/managing director. The subsidiaries involved in the context of these studies consist of different departments, and unlike the focus of my study, the R\&D unit in particular. Further, the sub-questions used in these measures were directed to the top managers in the subsidiary. They involved deep information on subsidiary functions such as on the subsidiary's ability to decide about restructuring, hiring and firing of top managers, or introduction of new products. While in my study, as the respondents are researchers/scientists/engineers in the $R \& D$ units who are unlikely to have any knowledge about the above, the measures could not be replicated.

Therefore, I adapt and use two items 'autonomy' and 'influence' to measure the power of the R\&D unit. The objective was to capture an individual employee's overall perception about the power of a subsidiary, which may influence his/her knowledge exchange decisions. Influence was measured by the perception of a respondent about "Ability of the subsidiary to influence HQ's decisions" using a 5-point Likert scale ' $1=$ very little' to ' $5=$ very much'. Autonomy was measured by an individual's perception about "Ability of the subsidiary to take decisions autonomously with less control from the HQ" using a 5-point Likert scale ' $1=$ very little' to '5=very much'.

The construct 'Subsidiary Power' is a combination of the indicators-'influence' and 'autonomy' (Borsboom, Mellenbergh, \& van Heerden, 2004). The causality is from the indicators to the construct. Variation in the item measures causes variation in the construct power (Bollen \& Lennox, 1991; Edwards \& Bagozzi, 2000). The items, influence and autonomy do not share a common theme and are not interchangeable. That is, adding or dropping an item may change the concept of power (Jarvis, MacKenzie, \& Podsakoff, 2003; Rossiter, 2002). Moreover, unlike the reflective indicators, the indicators-influence and 
autonomy may or may not be correlated (Diamantopoulos \& Siguaw, 2006). Therefore, from observation and assessment, it is found that it is a formative measurement construct (Coltman, Devinney, Midgley, \& Venaik, 2008).

For this study, the relative power of the own subsidiary of the respondent to the contact's subsidiary is referred to as 'subsidiary power', which is the predictor variable. The power of the own/contact's subsidiary is measured as a composite of two scores; influence and autonomy, by taking the mean values of these two scores. Then, the relative subsidiary power or 'subsidiary power' is calculated by subtracting the contact subsidiary's power from the power of the own subsidiary of the respondent. A positive value indicates a higher power of that own subsidiary and a negative value indicates lower power of own subsidiary, in relation to the subsidiaries of the contacts from/with whom the respondent seeks/shares.

As perception-based measures are considered more appropriate for research on human behaviour (Michailova \& Minbaeva, 2012), a subjective measure of subsidiary power is used instead of an objective measure.

\subsubsection{Mediator and moderators}

Organizational identification of the employees with their subsidiary was measured with six items on a five-point Likert scale adopted from Reade (2001b) and Smale et al. (2015). The six items assessed how much the employees identify with their local unit/subsidiary. A 5-point Likert scale is used where the scale ranges from ' $1=$ strongly disagree to $5=$ strongly agree'. The questions are as follows: 'The practices of this local unit/subsidiary are in line with my personal values', 'What this local unit/subsidiary stands for is important to me', 'My values and the values of the local unit/subsidiary that I work for are the same', 'I am proud to tell others that I am an employee of this company', 'In this company we have capable and sensible people in top management', and 'This company is likely to be successful in the future'.

Intra-MNE competition or inter-subsidiary competition was adapted from Hansen et al. (2005) and Kachra and White (2008). Inter-subsidiary/intra-MNE competition was measured with three perceptual items on a five-point Likert scale ' $1=$ no competition to $5=$ =very high competition'. The items measured perception of competition with the contact subsidiary with regards to funding from $\mathrm{HQ}$, common target market, and due to producing similar products and technologies. Again, a perceptual measure of intra-MNE competition is more appropriate than an objective measure as I examine human behaviour.

Cultural intelligence was measured using the measure developed by Thomas et al. (2015). It is a ten item measure using a five-point Likert scale ' $1=$ not at all to $5=$ extremely well'. The 
items measure various aspects of a person's awareness about cultural differences and his/her ability to deal with culturally different people. The following are the questions: 'I know the ways in which cultures around the world are different', 'I can give examples of cultural differences from my personal experience, reading, and so on', 'I enjoy talking with people from different cultures', 'I have the ability to accurately understand the feelings of people from other cultures', 'I sometimes try to understand people from another culture by imagining how something looks from their perspective', 'I can change my behaviour to suit different cultural situations and people', 'I accept delays without becoming upset when in different cultural situations and with culturally different people', 'I am aware of the cultural knowledge I use when interacting with someone from another culture', 'I think a lot about the influence that culture has on my behaviour and that of others who are culturally different', and 'I am aware that I need to plan my course of action when in different cultural situations and with culturally different people'.

Knowledge seeking/sharing is a second order construct having two first order factors, seeking/sharing amount and seeking/sharing use. Also, cultural intelligence is a second order construct having three first-order factors; knowledge, skill and metacognition. Intra-MNE competition and organizational identification are first order constructs. After performing second-order confirmatory factor analysis, the scores for all the variables, knowledge seeking/sharing, organizational identification, intra-MNE competition and cultural intelligence were imputed using AMOS version 23 in SPSS.

\subsubsection{Control variables}

Control variables increase the statistical power of the model, reduce the error terms and help rule out alternative explanations for the findings (Becker, 2005). The decision to include a variable as a control has to meet three criteria (Becker, 2005; Bono \& McNamara, 2011): Firstly, prior empirical research has established correlation between the variable and the dependent variable; secondly, it is expected to be correlated with the hypothesized independent variable(s); lastly, it is less important than the independent/ mediator/moderator variables Therefore, control variables are very carefully chosen so that the important ones are included and the superfluous ones are excluded.

Four individual level variables age, tenure, education, and task interdependence between the respondent and the contact he/she is seeking and sharing knowledge with, are used as control variables in the model. These variables may influence the dependent variable, interpersonal knowledge transfer. Similarity in demographic variables like age, tenure, and 
education between individuals can influence knowledge transfer between them. Individuals of similar age, tenure or education level often share similarity in their work, personal, and educational experiences which may facilitate knowledge transfers between them (Haas \& Cummings, 2015). These individuals are more likely to share similar values and attitudes due to shared experiences in the work organization which makes it easy for them to communicate. The other variable I controlled for is task-based interdependence between the respondent and the contacts so that the dependent variable captures voluntary knowledge seeking and sharing. This accounts for the extent to which the respondent and the contacts were required to communicate due to any formal task (Hass \& Cummings, 2015).

The ages of the respondent and the contacts were measured in years with a nominal scale ranging from ' $1=20$ to $29 \mathrm{yrs}, 2=30$ to $39 \mathrm{yrs}, 3=40$ to $49 \mathrm{yrs}, 4=50$ to $59 \mathrm{yrs}, 5=60$ and above yrs'. 'Age' was measured as relative age of the respondent using ' 0 ' if the respondent is younger, and ' 1 ' if the respondent is equal or older than the contact. As information on tenure of the contacts could not be received, tenure was measured only for the respondent, in years with a scale variable. Education was also measured with a scale variable. Task interdependence between the respondent and the contact was measured using the measure adapted from Haas and Cummings (2015). The respondents were asked to 'indicate the extent to which, he/she (the respondent) could not accomplish the task without information and materials from the contact' and 'indicate the extent to which the contact could not accomplish the task without information and materials from the respondent'. A 5-point Likert scale 1=not at all possible, $2=$ less possible, $3=$ =somewhat possible, $4=$ much possible, $5=$ very much possible was used.

However, some other variables such as cultural, geographic distance with the contacts, industry may also influence knowledge transfers between subsidiaries. In my study, most of the knowledge transfers occurred with contacts which were primarily from US, UK or Europe, which had similar cultural and geographic distance from India. Therefore, cultural and geographic distance were not included as controls. In organizational research, it is common to control for industry, as knowledge transfer in different industries is different. But, industry effect is offset by accounting for only R\&D units primarily from the manufacturing industry. Many studies have explored issues related to communication (Allen, 1977; Nobel \& Birkinshaw, 1998), knowledge flows (Teigland, Fey, \& Birkinshaw, 2000) between the R\&Ds and how MNEs engage in transnational learning through these global R\&D networks (Lam, 2003). Studies have also highlighted the dominance of interpersonal and informal communication and collaboration networks between the scientists from across different $R \& D$ centres for knowledge flows (Allen, 1977; Bouty, 2000; Kachra \& White, 2008; Kreiner \& 
Schultz, 1993). Therefore, intensive cross-border knowledge transfers is a common feature of any $R \& D$ unit irrespective of it being to any industry. As the objective of this study is to examine individual knowledge transfer behaviour, the effect of industry is less likely to have any impact.

\subsection{Data Collection Procedure}

\subsubsection{Sample frame}

The sampling frame consists of employees working in R\&D subsidiaries of foreign multinationals. Employees working in R\&D subsidiaries is an appropriate sample frame for this study. Although, traditionally, setting a R\&D facility in a foreign location was for the purpose of gaining internalization advantage to enable transfer of technology from home to host country, now-a-days, R\&D centres are sources of new knowledge for the whole organization as they are able to tap external knowledge (Feinberg \& Gupta, 2004). These R\&D centres which are responsible for generating new knowledge also become the centres of excellence, the source of valuable knowledge to the MNE. MNEs continuously create, transfer and exploit knowledge dispersed across different $R \& D$ units to gain competitive advantage (Bartlett \& Ghoshal, 1989).

In this study, R\&D subsidiaries are chosen over subsidiaries that control other functional departments. Many studies have explored issues related to communication (Allen, 1977; Nobel \& Birkinshaw, 1998), knowledge flows (Teigland et al., 2000) between R\&Ds and global R\&D networks (Lam, 2003). Mostly, studies have highlighted the prevalence of interpersonal informal communication and collaboration networks between scientists from across different R\&D centres for knowledge flows (Allen, 1977; Bouty, 2000; Kachra \& White, 2008; Kreiner $\&$ Schultz, 1993). Compared to other subsidiaries, the frequency and the amount of knowledge exchange are more between $R \& D$ units. Moreover, in $R \& D$ units, apart from the top executives, bench-level scientists or employees at the middle level also engage in cross-border knowledge exchanges as they receive requests for know-how from their counterparts in other subsidiaries (Kachra \& White, 2008). Therefore, R\&D subsidiaries provide an appropriate environment where cross-border knowledge exchange not only exists but thrives. So, employees working in R\&D units are an appropriate sample frame to study interpersonal crossborder knowledge exchange behaviour in the MNE.

In a study by Buckley et al. (2003), it was found that wholly owned subsidiaries (WOS) are faster and more efficient at achieving primary knowledge transfer (from headquarters to subsidiary) than JVs. Also, tacit technologies that are less codifiable and harder to teach, can 
be transferred at a lower cost to wholly owned subsidiaries than to third parties (Kogut \& Zander, 1993). Due to greater headquarters control, secured knowledge flows and full retention of the benefits of knowledge transfers with the MNE, WOS facilitate more intra-MNE knowledge inflows and outflows (Frost \& Zhou, 2005; Grosse, 1996; Wang et al., 2004). Therefore, wholly owned subsidiaries over joint ventures, alliances or any other forms of partnership are the subjects for the study. Thus, the sample frame consists of employees more specifically researchers/scientists/engineers in R\&D units of foreign MNEs belonging to any industrial sector in a particular country.

\subsubsection{Sample selection}

India is fast emerging as a major destination for MNEs for high-end R\&D projects (Deloitte, 2011; Gupta \& Gupta, 2014). It provides a fertile ground for studies on R\&D units. As per the latest report from US-based HfS Research, India leads with 30 per cent of the global ER\&D centre announcements from April to December 2015 (Aggarwal, 2016). R\&D centres in India are predominantly from the US (Bliese, Chan, \& Ployhart, 2007). As posited by Motohashi (2015), these R\&D units are considered at par with those in US, Europe or any other advanced countries. According to a report by Zinnov, a global consulting firm, by the end of 2015, there were 1,165 R\&D units established by 928 foreign MNEs in India with a workforce of approximately 323,000 scientists and engineers.

In a survey of foreign investment in R\&D in India during 2003-09, it was found that in terms of sectoral share, the largest share was in IT \& software $(50.36 \%)$, followed by aerospace (12.52\%), pharmaceuticals \& biotechnology (9.72\%), automobiles (9.27\%), machinery \& equipment $(3.01 \%)$, services $(2.62 \%)$, electronic components $(2.56 \%)$, chemicals $(1.25 \%)$ (Mrinalini, Nath, \& Sandhya, 2013). The target sample includes employees (technical staff), scientists/researchers/engineers working in R\&D units of foreign MNEs belonging to different industrial sectors in India.

I adopted a non-probability sampling approach of purposeful convenience sampling (Sekaran \& Bougie, 2016; Hair, Celsi, Money, Samouel, \& Page, 2016) because the target respondents need to meet the set specification of working as a researcher/scientist/engineer in a R\&D of a foreign MNE in India. It is difficult to identify and get access to these respondents. There are no databases available to contact the target respondents. Therefore, in the first stage, the $R \& D$ units are identified. As there are no available lists of R\&D units of foreign MNEs in India, I obtained the list of organizations which were granted patents originating from India 
during the period from 2011-2015(31 ${ }^{\text {st }}$ Dec) published by the United States Patent and Trademark Office (USPTO).

This list comprises the organizations and patent count where the first-named inventor listed on the patent grant is a resident of India. Out of a total of 256 organizations, 156 are foreign MNEs. The objective of selecting the sample from the USPTO list was to first identify those foreign MNE R\&Ds where knowledge exchanges are quite evident from the patents that they have been able to produce. Additionally, this also ensured that the MNEs selected, have atleast two or more R\&D units in different countries outside of India. This gives the respondent in the Indian unit choice (out of the other two subsidiaries) regarding engaging in cross- border knowledge exchange.

The next task was to identify the target respondents which are scientists/engineers/researchers working in these R\&D units. Challenges to collecting data in India has been highlighted in many studies. It is more difficult to find organised directories and databases of individuals and companies in India than in any other country (Krishnan \& Poulose, 2016). Moreover, considering non-response rates are comparatively higher in online surveys (Kwak \& Radler, 2002), I started with a drop-and-collect survey using paper questionnaires. For this, I contacted a manager from the top management team e.g. MD/R\&D manager in the target R\&Ds, who identified the respondents for the survey.

To supplement the above approach to access the respondents, an online survey method was also used. Electronic surveys have an advantage over others due to their low cost, quick turn-around time and wide reach (Dixon, 2007). As no formal database was available, the target respondents were searched online through the professional networking site 'LinkedIn'. The respondents were identified through their profile description on LinkedIn which matched the required target respondent sample profile. Key words used to conduct the search includes the name of the MNE R\&D, job titles: researcher/engineer/scientist.

\subsubsection{Data collection}

Data was collected using a questionnaire survey method. Two different approaches were used, a paper-based and an online survey. Initially, a paper-based pick and drop method was used. Later, to supplement the paper-based survey and collect adequate responses, an online survey was used. As put by Sax, Gilmartin, and Bryant (2003), an online survey is a methodological alternative to a paper questionnaire. The process of data collection through the surveys is documented below. 
For the paper-based survey, the head of the subsidiary holding profiles such as MD/CEO/Vice President was contacted. A meeting was scheduled wherein the purpose of the study and its implications to organizational management was explained and presented. Full confidentiality of the organization and anonymity of the survey was assured. The manager signed the consent of participation and gave approval for conducting the survey with employees of his organization. The survey questionnaire was then distributed to the employees identified by the subsidiary head, and collected on a later date. Routing the survey through the head of the organization is a proven way of data collection in India, as a request from a higher or other legitimate authority has a positive effect on employee involvement (Krishnan \& Poulose, 2016). Out of the total 333 responses, 193 were received through this approach.

The second approach through an online survey was adopted to supplement the paperbased approach. The online questionnaire was designed using the software tool 'Qualtrics'. Potential respondents were contacted on LinkedIn and sent a request for connecting. Compared to the western context, direct ways of data collection are more prevalent and effective in India (Krishnan \& Poulose, 2016). Direct interaction or personalised communication brings familiarity to the potential respondents and makes them receptive. Direct contact with respondents through LinkedIn helped establish reliability and credibility of the study.

Similar to the 'brief pre-notice letter' as recommended in a mail survey by Dillman (2000) in Tailored Design Method, I included a brief message along with the request for connecting. In the brief message, I mentioned about the study and my objective of connecting to the potential respondent. After they accepted my request, I sent them the details of my study with a cover letter and link to the online survey. The participant information sheet used as a cover letter is attached in Appendix 2. I assured them anonymity and confidentiality. Also, the question about the contact details of the respondents was kept optional. There was no way I could track the responses to identify the respondents. However, the organization the respondents belonged to was known as the organization name was made compulsory. Two weeks later, an email was sent thanking the ones who completed the survey. Along with the thank you email, the survey link was sent again for those who were yet to complete it. It was possible to identify them as the ones who had completed would inform me about the same. For those who had still not completed, I sent them a reminder email after about four weeks. 140 responses were received through this method. 


\subsection{Tests for Biases}

\subsubsection{Response bias}

This study uses two different methods of collecting data, a paper based and an online survey. In the early phase, the paper-based survey was used and in the later phase, the online survey was used. Using two different methods for the survey was necessary to increase the response rate and gain more coverage of the population spread across different parts of the country. But, paper-based vs online method may have an impact on the responses received. This would potentially make the survey biased. To assess any response bias issue due to this difference in survey method, an independent sample t-test is conducted to compare the responses of items of the dependent variables knowledge seeking and knowledge sharing for surveys conducted by the two methods. I compared 193 respondents who had filled in the paper questionnaire against 140 who completed online.

Non-response bias is another issue that needs to be considered. If the responses received are significantly different from the non-responses, it means the responses are not representative of the population. Therefore, it may significantly impact the results of the study by its effect on the external validity of the study (Viswesvaran, Barrick, \& Ones, 1993). Armstrong and Overton (1977) suggest that late respondents are often considered to be more representative of non-respondents than early respondents. Therefore, to check the non-response bias issue, a ttest comparing the two sets of responses, early and late, on the dependent variable is recommended (Peltokorpi \& Yamao, 2017). These 193 paper-based and 140 online responses also represent responses which were collected in the early and late phases of the survey respectively. As discussed, the online survey method was used in the latter half of the data collection phase to supplement the paper survey. Thus, the results from the t-test for paper and online survey is also used to assess non-response bias using early and late responses respectively. The test results are in section 5.4

\subsubsection{Common method variance}

My study aims to capture the perception or attitude of the respondents towards various constructs of interest. Variables which measure attitudes are most susceptible to common method variance (CMV). "It is the variance that is attributable to the measurement method rather than to the constructs the measures represent" (Podsakoff, MacKenzie, Lee, \& Podsakoff, 2003, p. 879). For example, self-report data can create false internal consistency between variables if respondents have a tendency to provide consistent answers to questions otherwise not related. It can inflate or deflate observed relationships between constructs which 
may lead to type I and II errors (Chang et al., 2010). In cross-sectional studies like this one, when individuals report their present and past internal states as well as behaviours related to those states at the same time, there is a possibility that method variance has inflated the observed correlations between the variables artifactually (Lindell \& Whitney, 2001).

To minimize the potential of CMV, Chang et al. (2010) recommends using some ex ante and ex post approaches as forwarded by Podsakoff et al. (2003). In this study, I have applied some of these recommended steps. At the design stage, I have mixed the order of questions and used complicated regression models which make it unlikely for respondents to visualize interactions and non-linear effects. Respondents were assured of the anonymity and confidentiality of the study. Great care was taken to remove any form of ambiguity or vagueness and not use any unfamiliar terms.

As an ex post approach, I have used two statistical approaches. At first, I have used Harman one-factor analysis to check whether variance in the data is attributable to a single factor. This method loads all constructs into an exploratory factor analysis to check if one single factor emerges, or if a majority of the covariance between the measures is accounted for by one general factor (Chang et al., 2010). Additionally, I have used Lindell and Whitney's (2001), partial correlation technique using a marker variable, "Fashion Consciousness" (Malhotra, Kim, \& Patil, 2006). A marker variable is a variable which is theoretically unrelated to at least one other variable in the study. As it is theoretically unrelated, it can be predicted that there is zero correlation between the variables. CMV is assessed based on the correlation between the marker variable and the theoretically unrelated variable (Malhotra, Kim, \& Patil, 2006). Following Lindell and Whitney's (2001), I checked the partial correlations between all the perceptual variables, controlling for "Fashion Consciousness".

The variable "Fashion Consciousness" is theoretically unrelated to any other variable of interest. The affective and evaluative elements in the variable 'fashion consciousness' stimulate the same response processes which are similar to those required in responding to the attitudinal and perceptive measures used for the other variables in the study (Chan, 2009) . Therefore, fashion consciousness is an ideal variable which I believe would be susceptible to CMV and would capture the common method bias well. Also, it will stimulate a similar response process as the substantive items because its items follow the same scale as other variables (5-point Likert scale) (Williams, Hartman, \& Cavazotte, 2010).

A three item measure to assess the fashion consciousness (Lertwannawit \& Mandhachitara, 2012) of the respondents used. It is measured with three items using a 5-point Likert scale. The items are, 'When I must choose between the two, I usually dress for fashion, 
not for comfort', 'An important part of my life and activities is dressing smartly', and 'A person should try to dress in style'. The mean score of the three items is used as a measure of fashion consciousness. The results are in section 5.8.

\subsection{Data Analysis Techniques}

\subsubsection{Preliminary analysis and screening}

\subsubsection{Missing data}

Missing data is an unavoidable component of any multivariate analysis. They can reduce the sample size available for analysis. When missing data are non-random, any statistical results based on that data could be biased. Therefore it is not only important to find out the extent of missing data, but also of primary concern is to identify the patterns and relationships underlying the missing data. So, when data is imputed or any other remedy is applied, it would help us maintain the original distribution of values (Hair, Black, Babin, \& Anderson, 2010).

Hair et al. (2010) suggested a four step process for identifying missing data and applying remedies. The first step is to determine the type of missing data. If the missing data are expected and part of the research design, they are ignorable and do not require any specific remedies. Whether the non-ignorable missing data are due to reasons known or unknown to the researchers, the next step is to determine the extent and pattern of missing data. The researcher needs to find out whether the amount of missing data is low enough to not affect the results. If it is low then we can use any of the remedies, or, missing data under $10 \%$ for an individual case or observation can generally be ignored and imputation can be avoided if we have a sufficient number of cases with no missing data for the selected analysis technique. Variables or cases with $50 \%$ or more missing data should be deleted. But, if the missing data is not low enough to not affect the results, we need to examine the degree of randomness present in the data which will then determine the appropriate remedies. Based on whether the missing data is 'Missing at Random' or 'Missing Completely at Random', different data imputation techniques are applicable. Missing data analysis is presented in section 5.2.

\subsubsection{Data coding}

The data collected from respondents was coded in Microsoft Excel. After coding the data, it was transferred to the Statistical Package for the Social Sciences (SPSS). All the items were coded in the range from 1 to 5 as per the codes allotted in the questionnaire and as discussed in the previous section. The variables 'Age' and 'Education' are recoded. 'Age' captures the relative age of the respondent with respect to the contact he/she is seeking from or sharing knowledge with. 'Age' is coded as 0 if the respondent is younger than the contact. It is coded 
1 if respondent is equal or older than the contact. 'Education' is coded as 0 if the respondent has an undergraduate degree and coded as 1 if he/she has a post-graduate degree.

\subsubsection{Checking for outliers}

Outliers are observations which are distinctly different from other observations. They appear as an unusually high or low value for a variable. Outliers can have a significant effect on any empirical analysis. Before categorizing them as beneficial or problematic for any analysis they must be viewed and evaluated within the context of the analysis and based on the type of information they are providing (Hair et al., 2010).

I detect univariate outliers using the method suggested by Hair et al. (2010). The data for each variable is first converted to standard scores which have a mean of 0 and a standard deviation (SD) of 1 . Then I examine the observations on each of the variables individually to identify cases exceeding \pm 2.5 standard deviations. I observed 6 cases in total. But Hair et al. (2010) recommends that for a larger sample size (greater than 80) we may increase the threshold value of the standard scores up to 4 . The analysis is presented in section 5.6.1.

\subsubsection{Sample size}

According to a popular method to determine minimum sample size needed for a study, a sample size need to be five times the number of variables included in the model (Tabachnick \& Fidell, 2007). The number of variables in my study is nine. Therefore, the sample size should be at least 45. Another method as suggested by Green (1991) is, to test an overall model, a minimum of $50+8 \mathrm{k}$ cases is required, where $\mathrm{k}$ is the number of predictors in the model. Further, he recommended that $104+\mathrm{k}$ cases are required for testing individual predictors. Based on these two calculations, he suggested using the greater value out of the two. Accordingly, in my study, I require 114 and 112 respectively. With respect to the above two methods, my sample of 333 cases is sufficient for this study.

However, for mediation analysis, I also refer to a table of sample sizes developed by Fritz and MacKinnon (2007). The table (Table 4.1) provides sample sizes needed to detect indirect effect, generated using the Percentile Bootstrap method, of certain sizes and to achieve 0.8 power. In calculating the sample sizes, they have used Cohen's (1988) criteria for small ( $2 \%$ of variance), medium (13\% variance) and large (26\% variance) effect sizes, values of 0.14 , 0.39 , and 0.59 respectively are chosen for ' $\alpha$ ' (path $\mathrm{X}$ to $\mathrm{M}$ ) and ' $\beta$ ' (path $\mathrm{M}$ to $\mathrm{Y}$ ) to form the indirect path $\alpha \beta$ sizes. Additionally, one more value 0.26 which is approximately halfway between the small and medium effects was also used to generate the table. Sample sizes with respect to 16 different indirect effects using combinations of these four values is tabulated 
below. The minimum sample size needed for small effects of $\alpha$ and $\beta$ to achieve 0.8 power is $558\{\alpha \beta=\mathrm{SS}=(.14)(.14)=.0196$. That is, $1.96 \%$ variance is explained which is slightly less than $2 \%$ (Cohen's small effect) . The minimum sample size needed for HH (between small and medium) is 162; for MM (medium) effect, it is 78. Finally, for a LL (large) effect size, a minimum sample size of 36 is needed to achieve 0.8 power. In my study, the 333 sample size is sufficient to detect a combination of ' $H$ ', ' $M$ ' and ' $L$ ' effect sizes to achieve a power of 0.8 .

Table 4.1Empirical estimates of sample sizes needed for 0.8 power using percentile bootstrap

\begin{tabular}{|c|c|c|c|c|c|c|c|c|c|c|c|c|c|c|c|c|}
\hline Method & SS & SH & SM & SL & HS & $\mathrm{HH}$ & HM & HL & MS & MH & MM & ML & LS & LH & LM & LL \\
\hline $\begin{array}{l}\text { Percentile } \\
\text { bootstrap }\end{array}$ & 558 & 412 & 406 & 398 & 414 & 162 & 126 & 122 & 404 & 124 & 78 & 59 & 401 & 123 & 59 & 36 \\
\hline
\end{tabular}

Source: Fritz and MacKinnon (2007).

\subsubsection{Confirmatory factor analysis}

Confirmatory factor analysis (CFA) is used for pre-existing validated scales. It helps test how well the measured variables have been able to represent the constructs (Hair et al., 2010, p. 668). This study used existing scales to measure the constructs, that is, the constructs are already established. Therefore, I performed CFA to assess the distinctiveness of the measures and finalise the measurement model. I verify the underlying dimensions of the latent factors and also identify the pattern of the relationship of the observed indicators with the factor (Brown, 2015). With CFA I find the extent to which the observed variables are linked to their underlying latent factors (Byrne, 2010). Also, CFA helps assessing the reliability and validity of the constructs.

CFA was conducted on the reflective measures where latent constructs cause the measured variables. CFAs were conducted separately for the two models, knowledge seeking and knowledge sharing. Model fit was assessed using values of $\mathrm{X}^{2}$, Root Mean Square Error of Approximation (RMSEA), Comparative Fit Index (CFI), PCLOSE, TLI (Tucker Lewis Index), Standardized Root Mean Residual (SRMR), Incremental Fit Index (IFI). The cut-off criteria is in the Table 4.2.

In the first step, CFA was run with all the observed variables which were grouped under the respective latent constructs. A minimum of three items per factor are taken to provide a minimum coverage of the construct's theoretical range and adequate identification. A threeindicator rule is applied where every factor has a minimum of three indicators (Hair et al., 
2010). Standard residuals less than 2.5 are acceptable. Residuals greater than 4.0 are unacceptable.

Table 4.2 Cut-off for fit indices for CFA

\begin{tabular}{lc}
\hline \multicolumn{1}{c}{ Indexes } & Cut-off \\
\hline X $/$ df & $\leq 2$ \\
TLI (Tucker-Lewis Index) & $\geq 0.95$ \\
CFI (Comparative fir index) & $\geq 0.95$ \\
GFI (Goodness-of-fit index) & $\geq 0.95$ \\
RMSEA(Root mean square errors of & $<0.06$ \\
approximation) & $>0.05$ \\
PCLOSE & $\geq 0.95$ \\
IFI (Incremental fit index) & For model comparison. \\
AIC (Akaike information criterion) & Smaller the value the better is \\
& the model \\
\hline
\end{tabular}

Source: Hair et al., (2010)

Modification indices (MI) provide information on potential cross-loadings that could exist if they are specified in the model. MI of approximately 4.0 or greater suggest that the fit of the model could be improved. Standardized factor loading below \pm 0.5 do not qualify as good items and are considered for deletion (Hair et al., 2010). After assessing the model, the model was re-specified by removing items with cross-loadings on more than one factor and then reestimated. Thus, I arrive at Model 2 which provides better fit to the data based on the above criteria. However, this Model 2 has a minimum of two indicators. A two indicator construct is allowed to be used when it is integrated into a CFA model with other constructs, as the required degrees of freedom to identify the overall model is provided by the extra degrees of freedom from the other constructs having more indicators (Hair et al., 2010).

Similarly, the three first level latent factors -Metacognition, Knowledge and Skill measure a second-order common latent construct, cultural intelligence (Thomas et al., 2015). Hair et al. (2010) suggests that there are some theoretical and empirical justifications to apply a higher order model for these factors. Theoretically, the constructs, Knowledge seeking/sharing and cultural intelligence have multiple conceptual layers. These constructs may be used for predicting similar other abstract constructs. The first order factors of amount and use of knowledge seeking/sharing, and metacognition, knowledge and skill, may influence other nomologically related constructs in the same way. Also, both the first-order and secondorder models meet the minimum conditions of identification and good measurement practices. Moreover, empirically, high correlation is found between the first order factors, between the amount and use of knowledge seeking/sharing; and between metacognition, knowledge and skill. This warrants the need to apply a second-order CFA model for the measurements. 
After conducting the CFA, scores for the latent factors in the two models, knowledge seeking and sharing, are imputed in SPSS using AMOS for analysing the data using regression analysis. These latent factors are, Knowledge Seeking/Sharing, Intra-MNE competition seeking/sharing, Identification and Cultural Intelligence. Results of the CFA are available in section 5.5.

Before using the measures for analysis, we need to ensure that the indicators or observed variables are able to measure and represent the concepts accurately and consistently. Therefore I check for reliability, which can be associated with consistency, validity, and accuracy, of the measures (Hair et al., 2016).

\subsubsection{Reliability}

A measure is considered reliable if its repeated calculation gives consistent scores (contingent on the construct being unchanged). For a multi-item scale measuring a construct to be reliable, the scores for individual items must be highly correlated (Hair et al., 2016). Reliability of such a summated scale, where there are multiple items for a construct, is measured through measuring internal consistency reliability. I used Cronbach $\alpha /$ coefficient $\alpha$ and Composite Reliability (CR) to check internal consistency reliability of the measures.

Composite Reliability assesses the internal consistency of a measure (Fornell \& Larcker, 1981). It is the reliability of a summated scale. It estimates the extent to which a set of latent construct indicators share in their measurement of a construct (Hair, Anderson, Tatham, \& Black, 1998). I also use Cronbach's $\alpha$, which is similar to composite reliability, but considered to be a less accurate approach than Composite Reliability. This is because Cronbach's $\alpha$ assumes that all items in the calculation are weighted equally. Whereas, CR is calculated based on each item's individual reliability, that is, each item is weighted separately (Hair et al., 2016). It draws on the standardized loadings and measurement error for each item. A popular rule of thumb is that 0.70 is an acceptable threshold for composite reliability, with each indicator reliability above 0.50 (Fornell \& Larcker, 1981). Also, a minimum score of 0.7 is acceptable for Cronbach's $\alpha$ (Hair, et al., 2016).

This calculator estimates composite reliability as:

$$
\begin{gathered}
\qquad R=\frac{\left(\sum \lambda_{i}\right)^{2}}{\left(\sum \lambda_{i}\right)^{2}+\left(\sum e_{i}\right)} \\
=\quad \underline{\text { Square of total standardized loadings }} \\
\text { Square of total standardized loadings + Total measurement error }
\end{gathered}
$$

Cronbach's $\alpha$ was calculated using SPSS. CR was calculated using Stats Tools Package (Gaskin, 2016). Results are in section 5.5.2.1. 


\subsubsection{Convergent and discriminant validity}

Validity is the extent to which a construct measures what it is supposed to measure (Hair et al., 2016, p. 257). The extent to which a set of measured items are able to reflect the theoretical latent construct that they are designed to measure, is called construct validity (Hair et al., 2010). Construct validity can be assessed through three checks-convergent validity, discriminant validity and nomological validity (Hair et al., 2010).

Convergent validity indicates how well observed variables are able to explain a latent factor. The indicators of a particular construct are said to converge well if they share a high proportion of variance in common (Hair et al., 2010). Convergent validity can be assessed through estimation of the factor loadings and Average Variance Extracted (AVE).

An observed variable or an indicator is said to converge if it's standardized factor loading is greater than 0.5 or ideally higher than 0.7 and significant (Hair et al., 2010). Convergent validity is also determined by the AVE. AVE reflects the overall amount of variance explained by the latent factor in its indicators/observed variables (Fornell \& Larcker, 1981; Hair et al., 1998, p. 612). It is the variance in the indicators explained by the common factor. Acceptable convergent validity is achieved when the average variance extracted is $>$ or $=0.5$ (Shook, Ketchen, Hult, \& Kacmar, 2004; Hair et al., 2010). AVE was calculated using the Stats Tools Package (Gaskin, 2016). Results are in section 5.5.2.2.

\section{$\mathrm{AVE}=\quad$ Sum of standardized loading square Sum of standardized Loading Square + Measurement error Measurement error $=1-(\text { Standardized Loading })^{2}$}

Discriminant validity indicates how the individual latent factors are distinct from one another. Discriminant validity is established when a construct is unique and captures the phenomena in a way not represented by other constructs in the measurement model (Chin, 1998). A discriminant validity issue arises when it is found that a latent factor is better explained by an observed variable from another latent factor than its own. To evaluate discriminant validity, two rules are followed. The first one is, AVE should be greater than the Maximum Shared Variance (MSV). The second is square root of AVE should be greater than the inter-construct correlation (Hair et al., 2010). The MSV is the variance that the latent factor shares with other factors in the model which explains how well the factor is explained by items outside the factor which are items of other factors (Hariri \& Roberts, 2015). MSV and correlations were generated using the Stats Tools Package (Gaskin, 2016). Results are presented in section 5.5.2.3. 


\subsubsection{Assumptions of data analysis}

\subsubsection{Linearity}

One assumption of OLS regression analysis is linearity of the relationship between the variables in a model. If this assumption is violated, it may jeopardize the meaningfulness of the interpretation of the regression coefficients (Darlington \& Hayes, 2017). As suggested by Hair et al. (2010), I tested the linearity of relationships between the dependent and independent variables through a scatterplot output of the residuals. I adopt the approach of running a multiple regression analysis (as we have multiple independent variables) and plotting the residuals. Any nonlinear part of the relationship will be indicated in the residual plot as the residuals reflect any part of the dependent variable that is unexplained. First I observe the residual plots of the combined effect of the independent variables on the dependent variable. Then I use the partial regression plots that show the relationship of a single independent variable to the dependent variable, controlling for the effect of all other independent variables. A curvilinear pattern indicates a nonlinear relationship between the variables. I observed the scatter plot of residuals by regressing the following relationships, the results of which are in section 5.6.2:

- Subsidiary Power, Identification, Competition, CQ and control variables on Knowledge Seeking

- Subsidiary Power, Identification, Competition, CQ and control variables on Knowledge Sharing

- Subsidiary power and controls on Identification (Knowledge Seeking)

- Subsidiary power and controls on Identification (Knowledge Sharing)

- Each independent variable with knowledge seeking

- Each independent variable with knowledge sharing

\subsubsection{Distribution of the data-normality}

I assessed the univariate normality of the continuous variables. Normality of the data is a fundamental assumption in multivariate analysis. I examined the distribution of each variable by its mean, median, standard deviation, minimum, maximum, skewness, and kurtosis. Normality of the distribution is assessed by the shape of the distribution which can be measured by skewness and kurtosis. Also, the Kolmogorov-Smirnov test is used to statistically test normality. When the Kolmogorov-Smirnov is significant $(\mathrm{p} \leq 0.05)$, it indicates that the data is not normally distributed (Field, 2009). However, as this test is less reliable in large samples 
(N>100) (Field, 2009), skewness and kurtosis are measured.

Skewness measures the departure from a symmetrical distribution (Hair et al., 2016, p. 336). For a sample of more than 300 , I look at the absolute value of skewness. Items with an absolute value of skewness score between -1 and +1 are considered normal (Pallant, 2010). When skewness values are greater than 1 and less than -1 , then we can say that it is a skewed distribution (Hair et al, 2016). If values are greater than 1, then it positively skewed. If values are less than 1, we call it negatively skewed. Kurtosis is the measure of a distribution's peakedness (or flatness) (Hair et al., 2016, p. 336). For a sample of more than 300, I look at the absolute value of kurtosis. Items with an absolute value of kurtosis score between -2 and 2 are considered normal (Pallant, 2010). A value greater than +2 signifies that the distribution curve is too peaked whereas, a value below -2 shows that it is flat (Hair et al., 2016) (Sposito, Hand, \& Skarpness,t 1983). Results are in section 5.6.3.

\subsubsection{Homoscedasticity}

The assumption of homoscedasticity is related to any dependence relationship between variables. It refers to the assumption that the level of variance in the dependent variable across values of the predictor/independent variable(s) remains equal. If the variance is unequal across different predictor values, the relationship is called heteroscedastic. A heteroscedastic relationship may result in the correlation coefficient underestimating/overestimating the strength of the association at different levels of the independent variable. While the association may look better at some levels, at other levels it may not be so (Hair et al, 2010; Bors, 2018). Also, it may affect the standard errors and make hypothesis tests too stringent or too insensitive (Hair et al, 2010, p. 75).

Homoscedasticity is examined from a visual analysis of the scatter plots of the dependent variables. Also, I examined the presence of heteroscedasticity through the Breusch-Pagan and Koenker test using a macro in SPSS developed by Daryanto (2013). A significance value $(\mathrm{p} \leq 0.05)$ indicates that homoscedasticity is violated (Doh, Bunyaratavej, \& Hahn, 2009). According to Hayes and Cai (2007), violation of the homoscedasticity assumption can affect the validity and power of statistical tests when using OLS regression. So they suggest the use of a heteroscedasticity-consistent standard error (HCSE) estimator of OLS parameter estimates (White, 1980; Hays \& Cai, 2007) to reduce the effects of heteroscedasticity. The results are in section 5.6.4. 


\subsubsection{Non-independence of errors}

OLS regression also assumes the errors in estimation are statistically independent. Nonindependence may affect the accuracy of the estimation of the standard errors (Hayes, 2018a). If the errors in estimation are independent, say for observations ' $i$ ' and ' $j$ ', it means, there is no information in the error in estimation of y for case ' $\mathrm{i}$ ' that could be used to estimate the error in estimation of $\mathrm{Y}$ for ' $\mathrm{j}$ ' case. This may occur due to failing to account for the common factor that may be present in subsets of cases and may be related to the dependent variable. In our study too, respondents belong to different MNE subsidiaries which may be referred to as different subclasses. For nested data, like these, where the respondents are nested within 40 different R\&Ds, the intraclass correlation (ICC) (1) is calculated to check for any clustering effect.

ICC (1) is the variance attributable to group membership (Raudenbush \& Bryk, 2002). A high ICC indicates higher similarity between members within the same R\&D and higher difference between groups. This would lead to violation of the assumption of independence of observations as the observations within the same group will then depend on some group characteristics. A one-way random effects ANOVA is conducted on the variables based on 40 group memberships. The results are in section 5.6.5. ICC (1) is calculated as

$$
\begin{aligned}
& \text { Mean } \mathrm{Sq}_{\mathrm{B}}-\text { Mean } \mathrm{Sq}_{\underline{w}} \\
& \text { Mean Sq в }+(\mathrm{k}+1) \text { Mean } \mathrm{Sq} \text { w } \\
& \text { Mean } \mathrm{Sq}_{\mathrm{B}}=\text { Mean square between } \\
& \text { Mean Sq w =Mean square within } \\
& \mathrm{K}=\text { size }
\end{aligned}
$$

\subsubsection{Multicollinearity}

Multicollinearity refers to the correlation among independent variables. Multicollinearity between the predictor variables makes it difficult to assess the individual importance of a predictor. If they are correlated, each predictor will have similar variance in the outcome, due to which it becomes difficult to find out the importance of any one predictor. Thus, the unique variance explained by each independent variable decreases and the shared prediction percentage rises (Hair et al, 2010; Field, 2018).

Multicollinearity is assessed by reviewing a correlation matrix with Pearson's correlation coefficients to identify any substantial correlation among all the predictor variables (continuous as well as dichotomous). A value greater than the threshold of 0.5 indicates high correlation (Hair et al., 1995).

Additionally, multicollinearity was tested by calculating the Variance Inflation factor (VIF) value and Tolerance $(\mathrm{T})$ statistic. VIF indicates whether a predictor has a strong 
relationship with the other predictors. VIF greater than 10 indicates multicollinearity (Myers, 1990; Field, 2018). Tolerance is defined as the amount of variability of the selected independent variable not explained by the other independent variables. T should be greater than 0.1 for no or little multicollinearity (Myers, 1990; Field, 2018). Results are in section 5.6.6.

\subsubsection{Test of main effects}

To test my models, I have used conditional process analysis with the help of the PROCESS macro for SPSS (Hayes, 2018). The PROCESS macro is a popular software frequently used by business researchers and now-a-days often appears in major business journals.

PROCESS v3.3 offers 92 pre-programmed models for estimation. The models used in my study are pre-programmed models in PROCESS. Unlike SEM, PROCESS requires only one line of SPSS code to estimate a model. It can produce all statistical test results which require combinations of different parameter estimates, such as index of moderated mediation, bootstrapping, etc. at one click. It does everything behind the scenes. This makes it simple and user friendly (Hayes, Montoya, \& Rockwood, 2017).

PROCESS uses observed variables and relies on Ordinary Least Square regression for analysis. There are still many controversies regarding estimation of interactions between latent variables and also the methods require meeting various assumptions (Marsh, Wen, Hau, \& Nagengast, 2013). This makes estimating interactions involving latent variables complicated and less trustable. Therefore, I have converted my latent variables to observed variables through computation of scores after conducting CFA.

It is argued that PROCESS and SEM produce similar results. It generally makes no difference as to whichever is used and therefore, the choice is inconsequential (Hayes, 2013; Hayes et al., 2017).

\subsubsection{Mediation effect}

I propose a mediation effect model (Baron \& Kenny, 1986) in which an independent variable, Subsidiary Power $(\mathrm{X})$, causes an intervening variable, Organizational Identification (M), which in turn causes the dependent variable, Knowledge seeking/sharing (Y). There are various approaches to testing hypotheses on mediation (Mackinnon, Lockwood, Hoffman, West, \& Sheets, 2002; Wood, Goodman, Beckmann, \& Cook, 2008). The causal-steps approach introduced by Baron and Kenny (1986) has been the most commonly used. This approach proposes four steps to demonstrate mediation which involve proving significant effects for all the paths in the model- effect of $\mathrm{X}$ on $\mathrm{M}$, effect of $\mathrm{M}$ on $\mathrm{Y}$, direct effect of $\mathrm{X}$ on $\mathrm{Y}$ and the total effect of $\mathrm{X}$ on $\mathrm{Y}$. Despite its popularity, the causal-steps approach is criticised for its low 
statistical power. It doesn't provide a joint estimate of all the conditions or a direct estimate of the size of the indirect effect of X on Y (Mackinnon et al., 2002).

The second approach is the difference in coefficients approach (Clogg, Petkova, \& Shihadeh, 1992) where the effect of mediation is assessed by comparing the relation between $\mathrm{X}$ and $\mathrm{Y}$ before and after adjustment for the mediator (Mackinnon et al., 2002). It is conducted by taking the difference between the total effect of $\mathrm{X}$ on $\mathrm{Y}$ and the direct effect of $\mathrm{X}$ on $\mathrm{Y}$ adjusted for M, and then dividing by the standard error of the difference (Fritz \& Mckinnon, 2007). To test for significance, this value is then compared against a $t$ distribution.

The third approach is the product-of-coefficient approach where the product of the coefficient of the path $\mathrm{X}$ to $\mathrm{M}$ and the coefficient of the path $\mathrm{M}$ to $\mathrm{Y}$ (adjusted for $\mathrm{X}$ ), is divided by the standard error of the product and then this value is compared to a standard normal distribution to test for significance (Mackinnon et al., 2002). The Sobel test (Sobel, 1982) is a product of coefficients test. This approach is criticised as the product of $\mathrm{X}$ on $\mathrm{M}$ and $\mathrm{M}$ on $\mathrm{Y}$ do not provide information about the size and significance of the indirect effect. Moreover, the Sobel test requires a normal distribution of the indirect effect. Therefore, the Sobel test provides a conservative estimate for small effect and sample sizes as they violate the normality assumption (Wood et al., 2008).

Bootstrapping technique has been suggested to provide a more robust test for confidence interval for testing the significance of the indirect effect (Shrout \& Bolger, 2002) and also higher power than the Sobel test (Hayes, 2018a). A bootstrap confidence interval is constructed by repeatedly taking a random sample of size ' $n$ ' from the original sample, with replacement, and estimating the indirect effect ' $a b$ ' in each resample (Hayes, 2018). The estimates of indirect effect derived from these large number of samples forms a bootstrap distribution. The percentile bootstrap test forms a $100(1-\omega) \%$ confidence interval by taking the indirect effect bootstrap estimates that correspond to the $\omega / 2$ and $1-\omega / 2$ percentiles of the bootstrap sample distribution, where $\omega=$ Type I error rate (Efron \& Tibshirani, 1993; Manly, 1997; Fritz \& MacKinnon, 2007). If this confidence interval does not contain zero, we can conclude that mediation has occurred.

The percentile bootstrap confidence interval method is a more widely recommended method for inference about the indirect effect in mediation with proper balancing between validity and power considerations (Hayes, 2018a). Although, bias-corrected and accelerated bootstrap confidence intervals are considered better than the percentile method, it has been found that they can slightly inflate the likelihood of a Type I error when the actual values of either path ' $a$ ' or path ' $b$ ' is zero (Hayes \& Scharkow, 2013). But as it is not possible to know 
if the actual ' $a$ ' or ' $b$ ' values is zero, the decision about whether to employ a bias-corrected method is difficult. Therefore, a percentile bootstrap method is the preferred method (Hayes, 2018a).

Thus, to test mediation effect, I use the percentile bootstrap confidence interval method. For this, I use the statistical tool PROCESS Macro v3.3 in SPSS 23 developed by Andrew Hayes (2018a) which produces bootstrap estimates of the indirect effect in mediation analysis. I apply 5000 bootstrap samples, which is considered sufficient (Hayes, 2018a). I also seed the random number generator at 1000. Random resampling of the same data produces confidence intervals which are not fixed (the endpoints vary every time the analysis is run). 'Seeding' helps fix these moving confidence interval endpoints by instructing the random number generator to start the sequence from a particular number, say 1000.

I use simple mediation analysis to examine how an employee's perception about subsidiary power (SP, X) can transmit its effect on the employee's cross-border knowledge exchange behaviour (Knowledge Seeking/Sharing, Y). The proposed intervening variable or the mediator is organizational identification (OI, M) of the employees towards their subsidiary. Therefore, I test the mediating effect of OI on the two relationships: i) Subsidiary power on knowledge seeking, and ii) Subsidiary power on knowledge sharing, using Model 4 in Process Macro (Hayes,2018a). The following is the statistical model in Figure 4.2 for hypotheses 1, 2 and 3. Mediation test results are in section 5.7.1.

The statistical diagram represents two equations

$$
\begin{aligned}
& \mathrm{M}=i_{\mathrm{M}}+a \mathrm{X}+a_{1} \operatorname{Cov}_{1}+a_{2} \operatorname{Cov}_{2}+a_{3} \operatorname{Cov}_{3}+a_{4} \operatorname{Cov}_{4}+e_{\mathrm{M}} \\
& \mathrm{Y}=i_{y}+c \mathrm{X}+b \mathrm{M}+b_{1} \operatorname{Cov}_{1}+b_{2} \operatorname{Cov}_{2}+b_{3} \operatorname{Cov}_{3}+b_{4} \operatorname{Cov}_{4}+e_{\mathrm{y}}
\end{aligned}
$$

a: regression coefficient of $\mathrm{X}$ on $\mathrm{Y}$.

b: regression coefficient of $\mathrm{M}$ on $\mathrm{Y}$.

$c^{\prime}$ : regression coefficient of $\mathrm{X}$ on $\mathrm{Y}$.

$\mathrm{Cov}_{1}$ : Age $\mathrm{Cov}_{2}$ : Tenure $\mathrm{Cov}_{3}$ : Education $\mathrm{Cov}_{4}$ : Task interdependence ab: Indirect effect of SPseek/share on KSeek/Share through OI. 


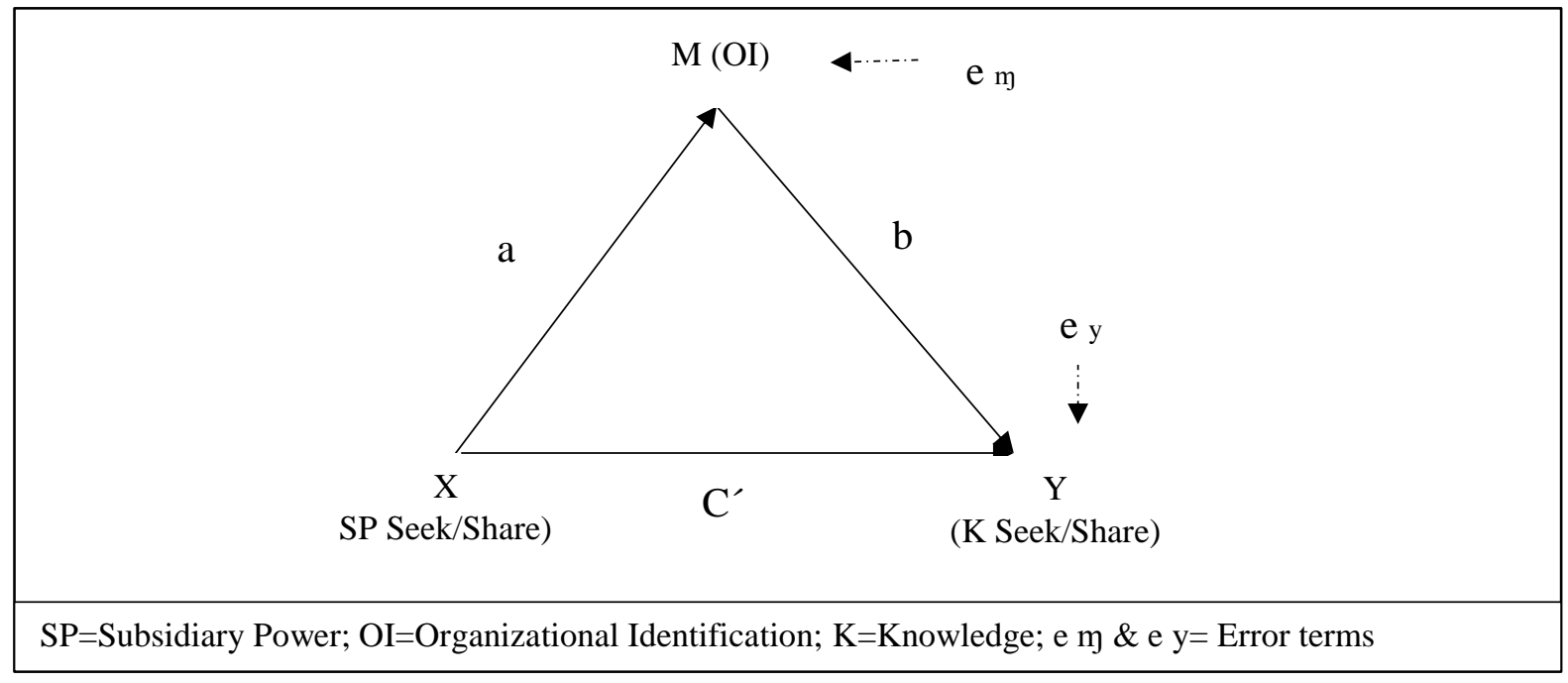

\subsubsection{Moderated mediation effect}

Moderation takes place when an effect is dependent on some other variable. If the size, sign or strength of the effect of $\mathrm{X}$ on $\mathrm{Y}$ depends on or can be predicted by $\mathrm{W}, \mathrm{W}$ is said to moderate the effect of $\mathrm{X}$ on $\mathrm{Y}$ and $\mathrm{W}$ is called the moderator (Hayes, 2018a). If an indirect effect (mediation) is contingent on a moderator, the effect is either a moderated mediation or mediated moderation. This type of analysis which examines the conditional nature of the mechanism/s and tests hypotheses about such contingent effects is called conditional process analysis (Hayes \& Rockwood, 2017).

Conditional process analysis can be conducted using the PROCESS Macro v3.3 in SPSS 23. Different combinations of moderation and mediation effects are programmed into different models in PROCESS. When either the direct or the indirect effect is moderated, PROCESS produces a table containing the conditional effects for various values of the moderator/s. It generates confidence intervals for the direct effects and bootstrap confidence intervals for conditional indirect effects (Hayes, 2018a).

In this study, the indirect effect (mediation) is conditional upon two moderators. If the indirect effect of $\mathrm{X}$ on $\mathrm{Y}$ through a mediator $\mathrm{M}$ is conditional upon two moderators $\mathrm{W}$ and $\mathrm{Z}$, this indirect effect which is a function of $\mathrm{W}$ and $\mathrm{Z}$, is called the index of partial moderated mediation which quantifies the linear relationship between a moderator and the indirect effect when a second moderator is held constant (Hayes, 2018b). Here, the indirect effect of subsidiary power on knowledge seeking and sharing through OI is conditional upon intra-MNE competition and cultural intelligence. The moderation effect takes place in the second stage of the mediation, on the direct effect of $\mathrm{OI}$ on knowledge seeking/sharing. This is a second stage 
dual moderated mediation model. Here, we examine whether one moderator moderates the indirect effect of subsidiary power on Knowledge Seek/Share independent of the effect of the other moderator.

Following Hayes (2018a); Dixon (2016); Peltokorpi (2015); Le and Kroll (2017) and Peltokorpi and Yamao (2017), I apply Process Model 16 to test the second stage dual moderated mediation model, as to whether an indirect effect depends on two moderators, intraMNE competition and cultural intelligence. Evidence of moderated mediation by a variable is reflected in the index of partial moderated mediation of that variable (Hayes, 2018b). If this index is statistically significant (different from zero), it implies that when the second moderator is held constant (at a particular value), the indirect effect is associated with the size of the first moderator (Hayes, 2015; Hayes \& Rockwood, 2017). If the index is not statistically different from zero or includes zero, then we infer that the indirect effect is unrelated to the size of the moderator when the second variable/moderator is held fixed (Hayes, 2018b). PROCESS automatically produces the index of partial moderated mediation for a model and provides a bootstrap confidence interval. As the sampling distribution of the indirect effects is likely to be non-normal, Hayes (2018a) recommends the use of bootstrap confidence interval to make a statistical inference about the indirect effect conditional on one moderator with the second moderator held fixed. The index on its own quantifies the relationship between the moderators and the indirect effect, without requiring to test for significance of the individual paths and interactions involved.

However, before testing the moderated mediation effect using Model 16, which contains the two moderators, following Le and Kroll (2017) and Peltokorpi and Yamao (2017), I elucidated the moderation effect of each individual moderator on the relationship that they both directly impact. The moderation effect of intra-MNE competition and cultural intelligence takes place in the second stage of the mediation, that is, on the direct effect of OI on knowledge seeking and sharing. Therefore, I use Process Model 1, which is a simple moderation model, to show the moderation effect of each moderator separately. After examining moderation of the direct relationship of the mediator OI on the dependent variable knowledge seeking and sharing, the dual moderated mediation with Model 16 is tested.

Once the second stage dual partial moderated mediation is established, the next step is to probe the interaction. Preacher, Rucker, and Hayes (2007) and Hayes (2015) recommend using the pick-a-point (or simple slopes) approach to probe the moderation of the indirect effect. If the moderator is a continuous variable, values of the moderator representing "low", "moderate" and "high" are picked and conditioned on those values of the moderator, the 
indirect effect is estimated. Inference about the conditional indirect effect is conducted using a bootstrap confidence interval to determine whether the effect differs from zero at one, some, or all of those three values picked. The three values may be at various levels of percentiles of the distribution $\left(16^{\text {th }}, 50^{\text {th }}\right.$, and $\left.84^{\text {th }}\right)$ or at a standard deviation below the mean, at the mean, and a standard deviation above the mean.

However, there are two moderators and the indirect effect is a function of two moderators. Although the second moderator is held fixed in the test of partial moderated mediation for the first moderator, we need to choose values for the second moderator as well. Say, in an indirect effect of $\mathrm{X}$ subsidiary power on $\mathrm{Y}$ knowledge seeking, $\mathrm{W}$ intra-MNE competition and $\mathrm{Z}$ cultural intelligence are the two moderators, to conduct an inference about the indirect effect of subsidiary power at some value of intra-MNE competition, we need to choose a value of cultural intelligence as well and vice versa. PROCESS can generate estimates for the indirect effect with both the moderators either at the $16^{\text {th }}, 50^{\text {th }}$ and $84^{\text {th }}$ percentiles or -1 $\mathrm{SD}$, Mean, and $+1 \mathrm{SD}$. For example, to examine the effect of intra-MNE competition, we may fix cultural intelligence at $50^{\text {th }}$ percentile or at the Mean. A confidence interval which does not include zero implies that the indirect effect is different for that value of intra-MNE competition with cultural intelligence fixed at a particular value. The statistical model to test our hypotheses is in Figure 4.3

Assuming that OI's effect on KSeek/Share is a linear function of Comp (W) and CQ (Z), the following equations represent the model.

$$
\begin{aligned}
\mathrm{M}= & i_{\mathrm{M}}+a \mathrm{X}+a_{1} \operatorname{Cov}_{1}+a_{2} \operatorname{Cov}_{2}+a_{3} \operatorname{Cov}_{3}+a_{4} \operatorname{Cov}_{4}+e_{\mathrm{M}} \\
\mathrm{Y}= & i_{y}+c \mathrm{X}+b_{1} \mathrm{M}+b_{2} \mathrm{~W}+b_{3} \mathrm{Z}+b_{4} \mathrm{MW}+b_{5} \mathrm{MZ}+b_{6} \operatorname{Cov}_{1}+b_{7} \operatorname{Cov}_{2}+b_{8} \operatorname{Cov}_{3}+b_{9} \operatorname{Cov}_{4}+\mathrm{e}_{\mathrm{y}} \\
= & i_{y}+c \mathrm{X}+b_{2} \mathrm{~W}+b_{3} \mathrm{Z}+\left(b_{1}+b_{4} \mathrm{~W}+b_{5} \mathrm{Z}\right) \mathrm{M}+b_{6} \operatorname{Cov}_{1}+b_{7} \operatorname{Cov}_{2}+b_{8} \operatorname{Cov}_{3}+b_{9} \operatorname{Cov}_{4}+\mathrm{e}_{\mathrm{y}} \\
& a=\text { the effect of } \mathrm{X} \text { on } \mathrm{M} \\
& b_{1}+b_{4} \mathrm{~W}+b_{5} \mathrm{Z}=\text { the conditional effect of } \mathrm{M} \text { on } \mathrm{Y} \text { which varies with } \mathrm{W} \text { and } \mathrm{Z} . \\
& \text { Indirect effect }=a\left(b_{1}+b_{4} \mathrm{~W}+b_{5} \mathrm{Z}\right)=a b_{1}+a b_{4} \mathrm{~W}+a b_{5} \mathrm{Z} \text {, which is a linear function of } \mathrm{W} \text { and } \mathrm{Z} . \\
& a b_{1}=\text { conditional indirect effect of } \mathrm{X} \text { on } \mathrm{Y} \text { when } \mathrm{W} \text { and } \mathrm{Z} \text { are both zeros (average). } \\
& a b_{4}=\text { Index of partial moderated mediation of the indirect effect by } \mathrm{W} . \\
& a b_{5}=\text { Index of partial moderated mediation of the indirect effect by } \mathrm{Z} .
\end{aligned}
$$




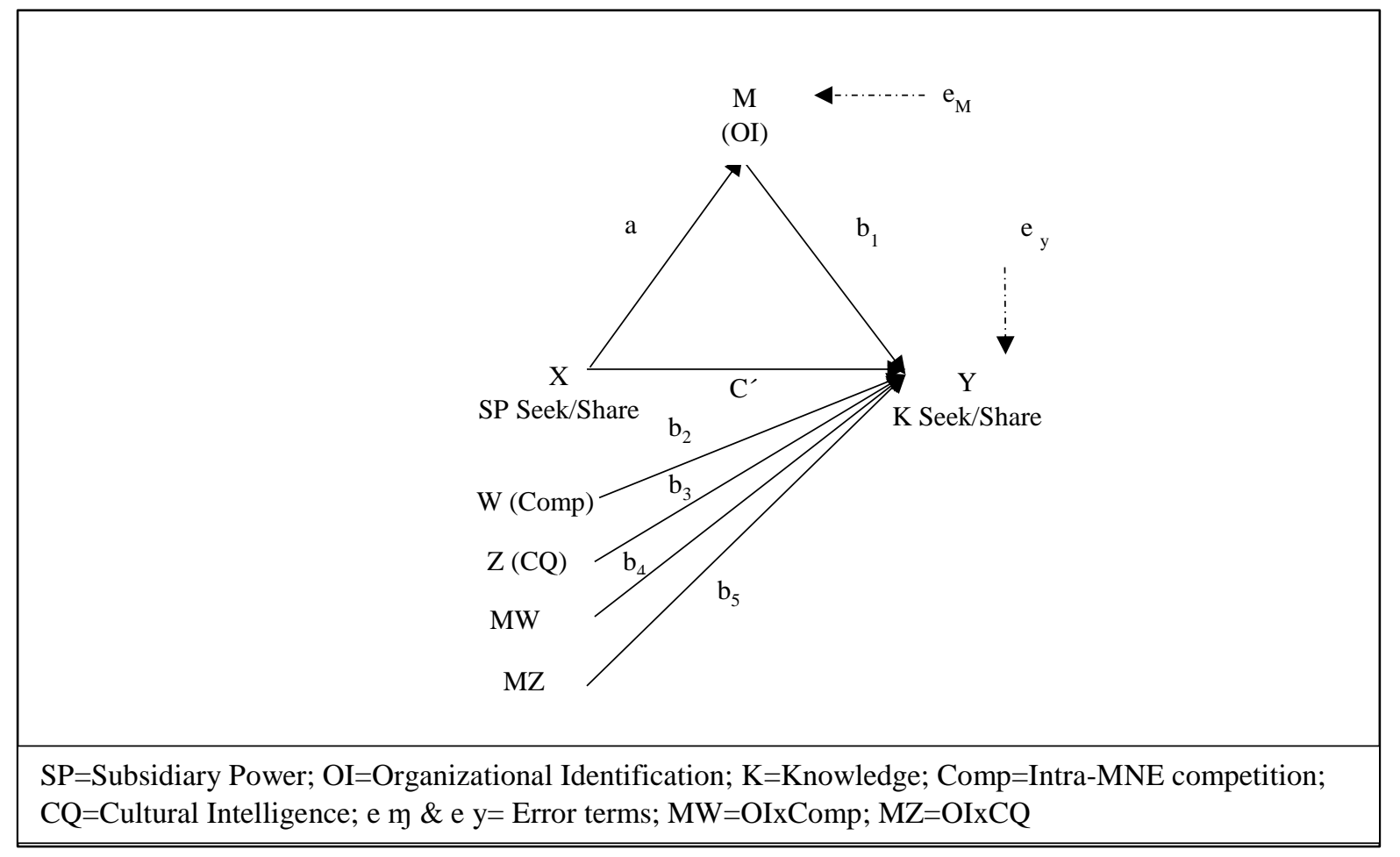

I have chosen to mean centre the variables so that the values are standardized. Although it is not necessary to mean centre as it does not have any effect on the test of interaction, standardization merely changes the metric of measurement so that one unit corresponds to one standard deviation and interpretation becomes easier (Hayes, 2013). The results are presented in section 5.7.2 and 5.7.3.

\subsubsection{Effect size}

Through regression analysis in Process, I arrive at estimates of the direct and indirect effects which are scaled in terms of the metrics of the independent variable $X$ and the dependent variable $Y$. Therefore, the absolute sizes of the direct and indirect effects do not reveal whether the effects are small or large. According to Hayes (2018a, p. 133), "the quantification of effect size in mediation analysis is an evolving area of thought and research". Various methods of effect size calculation have been proposed, such as, Ratio of the indirect effect to the total effect $\mathrm{P}_{\mathrm{M}}$ (Alwin \& Hauser, 1975; MacKinnon, Warsi, \& Dwyer, 1995); Ratio of the indirect to the direct effect $\mathrm{R}_{\mathrm{M}}$; Proportion of variance in $\mathrm{Y}$ explained by the indirect effect $\mathrm{R}^{2}$ med (Fairchild, MacKinnon, Toborga, \& Taylor, 2009); Kappa-Squared $\mathrm{K}^{2} \quad$ (Preacher \& Kelley, 2011). However, all these measures suffer from some inherent flaws which make them unsuitable for use as effect size measures (Hayes, 2018a). 
The effect size measures that PROCESS can produce are partially and completely standardized measures of indirect effect in mediation models with bootstrap confidence intervals. The partially standardized effect size $a b_{p s}$ expresses the indirect effect relative to the standard deviation of $\mathrm{Y}$. That is, the change in standard deviation in $\mathrm{Y}$, due to one unit change in $X$. The completely standardized effect $a b_{c s}$ calculates by standardizing the value of $X$ as well. That is, the indirect effect is expressed as the change in standard deviation in Y due to change in one standard deviation in X. PROCESS produces the effect size for indirect effect in mediation model only (not available for moderated mediation). The results are in section 5.7.1.

\subsection{Summary}

This study uses a post-positivism approach where data is collected through drop and pick, and online surveys. The collected data was cleaned, coded and screened for preliminary analysis. Then it was analysed using Confirmatory Factor Analysis and Conditional Process Analysis using the PROCESS macro in SPSS. The preliminary data analysis and main tests techniques are discussed in detail in this chapter. In the next chapter, the actual data analysis and results are presented. 


\section{Chapter 5: Data Analysis \& Results}

\subsection{Introduction}

This chapter explains the analysis related to the data collected. The analysis begins with data cleaning and preliminary examination of the data. This is followed by confirmatory factor analysis, where the reliability and validity of the constructs are also examined. Next, the assumptions of the analysis are examined. This is followed by presenting the results and robustness of the data analysis. Lastly, the chapter summary is provided.

\subsection{Missing Data}

Two variables, 'tenure of the contact seeking from' and 'tenure of the contact sharing with' have $64(19 \%)$ and 89 (26\%) missing values respectively out of 333 cases. According to Hair et al. (2010, p. 48), variables with as little as $15 \%$ missing data are candidates for deletion. Therefore, these two variables are dropped from the analysis. Out of the remaining useable variables, the maximum number of missing data 4.4\% (15 missing out of 333) was found for the variable 'tenure of the respondent'. The missing data for this variable was ignored as it was less than $10 \%$ (Hair et al., 2010).

Out of the 333 total number of cases under observation, 23 cases $(7 \%)$ for the knowledge seeking model analysis and 36 cases (10\%) for the knowledge sharing model analysis have missing values. The PROCESS Macro requires complete data for analysis. It automatically detects and analyses only the cases with complete data. Therefore for data analysis PROCESS used 310 cases for the seeking model and 297 cases for the sharing model, which contains complete data. Since the number of missing values is less than $10 \%$, I decided 
to ignore the missing values and not impute those (Hair et al., 2010). The missing data is presented in Table 1 (Appendix 3).

\subsection{Sample Characteristics}

The data for this study was collected through questionnaire survey in India. The respondents are employees working at R\&D centres of foreign MNEs in India. Data was collected from 40 different R\&D subsidiaries of 40 different MNEs having headquarters in 11 different countries. The respondents are primarily working as engineers or scientists or researchers at these R\&D units. They hold various positions ranging from senior engineer/chief engineer/managers/heads of innovations/technology specialists/technology managers etc. Most of them hold a minimum engineering graduate qualification (Bachelors of Engineering/Bachelors of Technology). The bench-level scientists who receive requests for know-how from R\&D scientists, both inside and outside the firm, are considered appropriate for such studies (Kachra \& White, 2008). An additional criterion for selection of individual respondents was to ensure that none of these individual employees are members of the top management (Schrader, 1991). This is to ensure that the response is representative of the employees in the subsidiary, not the unit manager. The detailed sample characteristics is presented in Table 5.1.

Table 5.1 Sample characteristics of respondents

\begin{tabular}{lll}
\hline & R\&D characteristics & \\
\hline Number of Organizations & Manufacturing & 40 \\
Industry & Services & $92 \%$ \\
Home Country & USA & $8 \%$ \\
& Europe \& UK & $40 \%$ \\
& Asia-China, Japan, Korea & $42 \%$ \\
\hline & Respondent characteristics & $18 \%$ \\
\hline Number of respondents & & \\
Gender & Male & 333 \\
& Female & $97 \%$ \\
Age & $20-29$ & $3 \%$ \\
& $30-39$ & $4 \%$ \\
& $40-49$ & $26 \%$ \\
Qualification & $50-59$ & $51 \%$ \\
& 60 and above & $16 \%$ \\
Tenure & Graduate & $3 \%$ \\
Region & Post-Graduate & $44 \%$ \\
& $\leq 5 y e a r s$ & $56 \%$ \\
\hline
\end{tabular}


All the respondents are Indian nationals. Out of 333 respondents 97\% were males and only $3 \%$ females. About $50 \%$ of the respondents are in the middle age group (40-49 years). The number of graduates and post-graduates is approximately similar, $44 \%$ and $56 \%$ respectively. The respondents belong to different states in India: 53\% were from southern India whereas $47 \%$ from the rest of India. Although the first language of the majority of the respondents (almost all) is a native Indian language, it is common that everyone could read and write in English. English is one of the two languages (the other being Hindi) which is used for official purposes in India.

These 333 respondents were employed by different R\&D centres of MNEs. A total of 40 MNEs or R\&D units were listed. They are primarily in the manufacturing sector (92\%). The majority of the MNEs are headquartered in the US, UK and Europe (82\%). The remaining 18\% are from Asia.

\subsection{Response Bias}

Out of 333 survey responses, 193 responses were based on a paper based questionnaire survey while 140 were collected through an online survey. The t-test comparison of eight items of the dependent variables of the survey, based on paper vs online are presented in Table 2 and 3 (Appendix 3). A two-tailed significance value at $\mathrm{p}>0.05$ indicates no difference between the groups compared. As discussed in the research design chapter, the online survey method was used at the latter phase of the data collection to supplement the paper-based survey. Therefore, these results are also representative of non-response bias taking the paper survey as early and the online survey as late responses.

As observed, for knowledge seeking, in Table 2 (Appendix 3), the first four items measuring the amount of knowledge seeking are not different for the two groups, $p>0.05$. But, the last four items measuring the use of knowledge seeking are found to be different, $\mathrm{p}<0.05$. For knowledge sharing, Table 3 (Appendix 3), all eight items measuring the amount and use of knowledge sharing have a significance value $p>0.05$, which indicates that the two sets are not different and non-response bias is not present for knowledge sharing. However, for the knowledge seeking, a response-bias was found for the last four items out of eight. For the first four items, response-bias was not found. Overall, it can be safely argued that the threat of nonresponse bias is absent. 


\subsection{Confirmatory Factor Analysis}

\subsubsection{First-order CFA models}

The full model, which is model 1, comprising of all 27 items (observed variables) related to 7 latent constructs each for knowledge seeking and sharing as used in the questionnaire are in Figure 1 (Appendix 4). I checked the factor loadings, fit indices, standardized residuals and the modification indices (MI) in model 1. After assessing the models, the model was re-specified by removing items with cross-loadings on more than one factor and then the model was reestimated. Based on the MI report eight items (Q1.5, Q1.8, Q1.9, Q1.12, Q1.45, Q1.46, Q1.49, and Q1.56) having high covariance between them were removed from the knowledge seeking model; similarly, eight items (Q1.26, Q1.29, Q1.30, Q1.33, Q1.45, Q1.46, Q1.49, and Q1.56) were removed from the knowledge sharing model. Thus, I arrived at model 2 for each of knowledge seeking and sharing as in Figure 2 (Appendix 4). Model 2 is a better fitting model than model 1. Model 2 consists of 19 observed items, with 7 latent constructs for each of knowledge seeking and sharing. Findings for fit indices are in Table 5.2. Both model 1 and 2 are first-order models. Although model 2 provides a better fit to the data, a second-order model, model 3, in Figure 5.1, is generated due to higher correlation between the first-order latent constructs and following some established literature.

\subsubsection{Second-order CFA models}

Following literature, and theoretical and empirical reasoning, a second-order model was constructed for both knowledge seeking and sharing (see Figure 5.1). A higher correlation between the first order latent constructs are observed (Brown, 2015). In the knowledge seeking model, a higher correlation is observed between Seek Amount and Seek Use (0.842), Metacognition and Knowledge (0.665), Knowledge and Skill (0.656), and between Metacognition and Skill (0.867). Therefore, a second-order construct knowledge seeking is created for the two first-order constructs- seek amount and seek use. The second-order construct Cultural intelligence was created for the first-order factors, metacognition, knowledge and skill as suggested by Thomas et al., (2011).

Similarly, in the knowledge sharing model, higher correlation was observed between Share Amount and Share Use (0.852), metacognition and knowledge (0.670), knowledge and skill (0.669), metacognition and skill (0.862). Thus, the second-order constructs of knowledge sharing and cultural intelligence were constructed for the sharing model as well.

In the knowledge seeking model, all item loadings were greater than 0.56 , with the majority of them exceeding 0.71 with significance at 0.000 level (Byrne, 2001). CMIN/DF 
1.859, Comparative Fit Index (CFI) 0.943, Tucker-Lewis Index (TLI) 0.931, Root Mean Square of Approximation (RMSEA) 0.051, PCLOSE 0.428, NFI 0.886, IFI 0.944. The model with the lowest AIC value among all models specified for a data set is the best fit model (Burnham \& Anderson, 2004). AIC of the final second-order model is 397.982 whereas the previous models were 399.220 and 1066.759 .

In the knowledge sharing model, all item loadings were greater than .57 , with the majority of them exceeding .71 with significance at .000 level (Byrne, 2001). CMIN/DF 1.90, CFI .94, RMSEA .052, PCLOSE .349, TLI .927, NFI .883, IFI .941. AIC of the final second order model is 403.918 whereas the previous models were 405.168 and 1071.496.

With Model 3, the second-order models for each of knowledge seeking and sharing, a superior fit is achieved than Model 2. Also, as the second order models do not result in a significant decrease in the model fit, it may be concluded that the higher order models have been able to explain the high correlation in the first order factors well. Table 5.3 presents all the factor loadings for the observed and latent variables.

\subsubsection{Reliability}

Reliability is assessed through Cronbach's $\alpha$ and Construct Reliability. Construct Reliability for all the latent factors are greater than 0.70 . Also Cronbach's $\alpha$ is greater than 0.70 for all the scales except the first order factors CQ knowledge 0.65 and CQ Metacognition 0.69 for both seeking and sharing models. Values ranging between 0.60 and 0.70 exhibit moderate strength of association and are acceptable (Hair et al., 2016, p.255). Cronbach's $\alpha$ is sensitive to the number of items in a scale. As these factors are two item scale, so $\alpha$ is slightly lower. As Hair et al. (2010) suggests, a two item construct can be used when it is integrated into a CFA model with other constructs as the required degrees of freedom to identify the overall model are provided by the extra degrees of freedom from the other constructs having more indicators. The results are presented in Table 5.3.

\subsubsection{Convergent validity}

Convergent validity is assessed through factor loadings and the Average Variance Extracted (AVE). As presented in Table 5.3, all standard factor loadings are above 0.56 which is above the criteria of 0.50 (Hair et al., 2010). The AVE values for all the latent constructs in both the knowledge seeking and knowledge sharing models have been found to be greater than 0.50 . AVE is the variance in the indicators explained by the common factor. Acceptable convergent validity is achieved when the average variance extracted is $\geq 0.50$ (Shook et al., 2004; Hair, et 
al., 2010), which shows that at least $50 \%$ of the variance in the observed variables is due to the latent construct. The AVE values are presented in the Table 5.3.

\subsubsection{Discriminant validity}

Discriminant validity is examined through AVE and Maximum Shared Variance (MSV). All constructs have an AVE of at least 0.50 (Fornell \& Larcker 1981). All the AVE values are greater than the MSVs. Also, the square-root of AVE is greater than the correlation between any pair of latent constructs (Hair et al., 2010). The AVE and MSV values are in Table 5.3. The square root of AVE values are presented in the correlation table, in Table 5.8 and Table 5.9 (diagonally in bold). 
Table 5.2 Model fit indices of CFA

\begin{tabular}{|c|c|c|c|c|c|c|c|}
\hline \multirow[b]{2}{*}{ Indexes } & \multicolumn{4}{|c|}{ CFA for Knowledge Seeking model } & \multicolumn{3}{|c|}{ CFA for knowledge sharing model } \\
\hline & Cut-off & $\begin{array}{l}\text { First order } \\
\text { Model } 1\end{array}$ & $\begin{array}{l}\text { First order } \\
\text { Model } 2\end{array}$ & $\begin{array}{l}\text { Second order } \\
\text { Model } 3 \\
\text { Final model }\end{array}$ & $\begin{array}{l}\text { First order } \\
\text { Model } 1\end{array}$ & $\begin{array}{l}\text { First order } \\
\text { Model } 2\end{array}$ & $\begin{array}{c}\text { Second order } \\
\text { Model } 3 \\
\text { Final model }\end{array}$ \\
\hline$X^{2}$ & $\begin{array}{c}\text { Significant p- } \\
\text { values expected }\end{array}$ & $862.76(<.05)$ & $243.22(<.05)$ & $263.98(<.05)$ & $867.49(<.05)$ & $249.16(<.05)$ & $267.92(<.05)$ \\
\hline$X^{2} / d f$ & $\leq 3$ & 2.84 & 1.85 & 1.85 & 2.86 & 1.90 & 1.90 \\
\hline TLI (Tucker-Lewis Index) & $\geq .92$ & .820 & .931 & .931 & .810 & .927 & .927 \\
\hline CFI (Comparative fit index) & $\geq .92$ & .845 & .948 & .943 & .836 & .944 & .940 \\
\hline $\begin{array}{l}\text { RMSEA(Root mean square errors } \\
\text { of approximation) }\end{array}$ & $<.07$ & .075 & .051 & .051 & .075 & .052 & .052 \\
\hline PCLOSE & $>.05$ & .000 & .435 & .428 & .000 & .350 & .349 \\
\hline IFI (Incremental fit index) & $\geq .95$ & .847 & .948 & .944 & .838 & .945 & .941 \\
\hline AIC (Akaike information criterion) & $\begin{array}{c}\text { For model } \\
\text { comparison. } \\
\text { Smaller the value } \\
\text { the better is the } \\
\text { model }\end{array}$ & 1066.759 & 399.220 & 397.982 & 1071.496 & 405.166 & 403.918 \\
\hline
\end{tabular}

Source: Cut-off values from Hair et al. (2010, p.672) 
Figure 5.1 Second-order CFA Model 3 (final model)
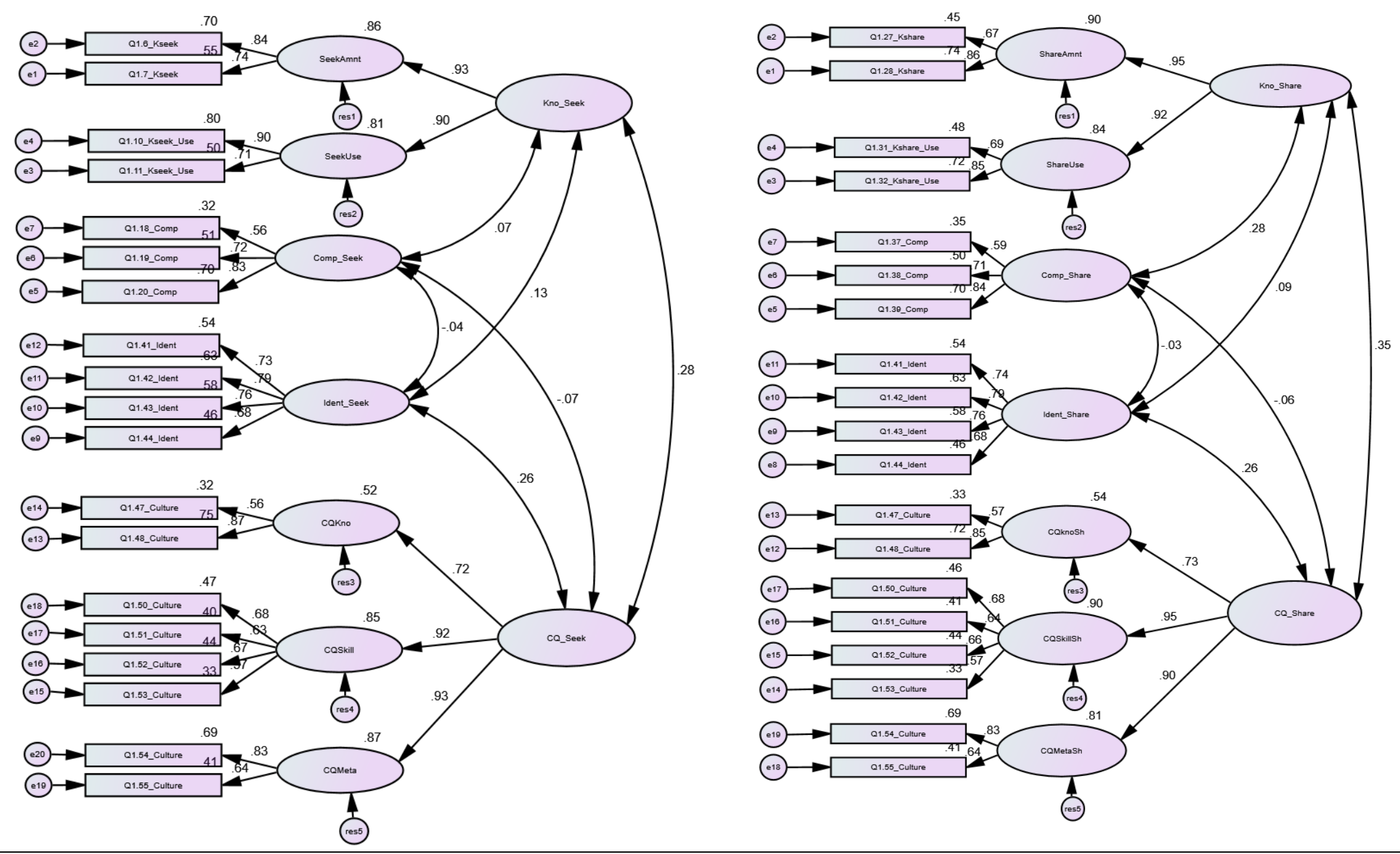
Table 5.3 Final measurement model item loadings, and reliability and validity test results

\begin{tabular}{|c|c|c|c|}
\hline $\begin{array}{c}\text { Knowledge Seeking Second-Order } \\
\text { Final model Model } 3\end{array}$ & $\begin{array}{c}\text { Factor } \\
\text { Loadings }\end{array}$ & $\begin{array}{c}\text { Knowledge Sharing Second-Order } \\
\text { Final model Model } 3\end{array}$ & $\begin{array}{c}\text { Factor } \\
\text { Loadings }\end{array}$ \\
\hline $\begin{array}{l}\text { Knowledge Seeking Second Order } \\
\alpha=.958(.839) \mathrm{CR}=.910 \mathrm{AVE}=.836 \mathrm{MSV}=.07\end{array}$ & & $\begin{array}{l}\text { Knowledge Sharing Second Order } \\
\alpha=.974(.839) \mathrm{CR}=.931 \mathrm{AVE}=.872 \mathrm{MSV}=.120\end{array}$ & \\
\hline Amount of knowledge received $\alpha=.764$ & .927 & Amount of knowledge shared $\alpha=.728$ & .950 \\
\hline Amount of advice & .840 & Amount of advice & .668 \\
\hline Amount of personal insights & .741 & Amount of personal insights & .857 \\
\hline Use of knowledge received $\quad \alpha=.778$ & .901 & Use of knowledge shared & .917 \\
\hline Use of advice & .895 & Use of advice & .693 \\
\hline Use of personal insights & .710 & Use of personal insights & .851 \\
\hline $\begin{array}{l}\text { Competition Seeking } \\
\alpha=.741 \mathrm{CR}=.751 \mathrm{AVE}=.508 \mathrm{MSV}=.005\end{array}$ & & $\begin{array}{l}\text { Competition Sharing } \\
\alpha=.750 \mathrm{CR}=.758 \mathrm{AVE}=.515 \mathrm{MSV}=.081\end{array}$ & \\
\hline Competition for funding & .562 & Competition for funding & .588 \\
\hline Competition for markets & .716 & Competition for markets & .706 \\
\hline $\begin{array}{l}\text { Competition due to products and } \\
\text { technologies }\end{array}$ & .834 & $\begin{array}{l}\text { Competition due to products and } \\
\text { technologies }\end{array}$ & .838 \\
\hline $\begin{array}{l}\text { Identification Seeking } \\
\alpha=.830 \mathrm{CR}=.831 \mathrm{AVE}=.552 \mathrm{MSV}=.066\end{array}$ & & $\begin{array}{l}\text { Identification Sharing } \\
\alpha=.830 \mathrm{CR}=.831 \mathrm{AVE}=.552 \mathrm{MSV}=.069\end{array}$ & \\
\hline Practices in line with personal values & .735 & Practices in line with personal values & .736 \\
\hline Importance of subsidiary & .794 & Importance of subsidiary & .794 \\
\hline Similar values & .762 & Similar values & .761 \\
\hline Proud to work & .677 & Proud to work & .676 \\
\hline $\begin{array}{l}\text { Cultural Intelligence Seeking Second Orde } \\
\alpha=.932 \text { (.826) } \mathrm{CR}=.896 \mathrm{AVE}=.744 \mathrm{MSV}=.0\end{array}$ & & $\begin{array}{l}\text { Cultural Intelligence Sharing Second Order } \\
\alpha=.936(.826) \mathrm{CR}=.898 \mathrm{AVE}=.748 \mathrm{MSV}=.120\end{array}$ & \\
\hline Knowledge & .720 & Knowledge & .732 \\
\hline Aware of cultural differences & .562 & Aware of cultural differences & .575 \\
\hline $\begin{array}{l}\text { Can give examples of differences } \\
\text { from experience, reading }\end{array}$ & .866 & $\begin{array}{l}\text { Give examples of differences from } \\
\text { experience, reading }\end{array}$ & .846 \\
\hline $\begin{array}{ll}\text { Skill } & \alpha=.728\end{array}$ & .921 & Skill $\alpha=.728$ & .950 \\
\hline Understand their feelings & .683 & Understand their feelings & .681 \\
\hline $\begin{array}{l}\text { Try to understand from their } \\
\text { perspective }\end{array}$ & .630 & $\begin{array}{l}\text { Try to understand from their } \\
\text { perspective }\end{array}$ & .637 \\
\hline $\begin{array}{l}\text { Can change behaviour to suit different } \\
\text { culture }\end{array}$ & .666 & $\begin{array}{l}\text { Can change behaviour to suit different } \\
\text { culture }\end{array}$ & .662 \\
\hline $\begin{array}{l}\text { Accept delays when with different } \\
\text { culture }\end{array}$ & .573 & $\begin{array}{l}\text { Accept delays when with different } \\
\text { culture }\end{array}$ & .572 \\
\hline $\begin{array}{ll}\text { Metacognition } & \alpha=.695\end{array}$ & .931 & Metacognition $\quad \alpha=.695$ & .931 \\
\hline Aware of cultural knowledge used & .829 & Aware of cultural knowledge used & .832 \\
\hline $\begin{array}{l}\text { Think a lot about influence of culture } \\
\text { on behaviour }\end{array}$ & .644 & $\begin{array}{l}\text { Think a lot about influence of culture } \\
\text { on behaviour }\end{array}$ & .642 \\
\hline
\end{tabular}




\subsection{Outliers \& Assumptions}

\subsubsection{Outliers}

One case in the variable 'organizational identification' is detected as an outlier. It has a value of 4.6, which just exceeds the threshold of 4 standard deviations for a larger sample as suggested by Hair et al. (2010). Hair et al. (2010, p. 67), recommends that outliers should be retained unless they are truly aberrant and not representative of any observations in the population. If they are representative of the population, they should be retained to ensure generalizability to the entire population. The above observation which just exceeds the threshold represents a value for organizational identification of an employee which is within the limits of the interval scale ( 1 to 5 ) that is used to measure the variable. This case, although an outlier among the observations, is representative of a segment of population. Therefore, I have decided to retain it. Table 5.4 presents the outliers.

Table 5.4 Identification of outliers

\begin{tabular}{lcc}
\hline \multicolumn{1}{c}{ Variable } & Number of cases exceeding & Number of cases exceeding \\
& \pm 2.5 & \pm 4 \\
\hline Subsidiary Power (Knowledge Seeking) & 1 & - \\
Subsidiary Power (Knowledge Sharing) & 1 & - \\
Identification (Knowledge seeking \& sharing) & 3 & 1 \\
Knowledge Sharing & 1 & - \\
\hline
\end{tabular}

\subsubsection{Linearity}

Linearity of the variables was tested by plotting scatterplot output of the residuals (Hair et al., 2010). Any nonlinear part of the relationship will be indicated in the residual plot as the residuals reflect any part of the dependent variable that is unexplained. A curvilinear pattern indicates a nonlinear relationship between the variables. A residual plot of the combined effect of the independent variables on the dependent variable was observed in Figure 5a (Appendix 5). Also, partial regression plots which show the relationship of a single independent variable to the dependent variable, controlling for the effect of all other independent variables was examined in Figure 5b (Appendix 5). A visual inspection indicated a linear relationship between the dependent and independent variables. 


\subsubsection{Normality}

The shape of the distribution of each variable was assessed by the values of KolmogorovSmirnov test (K-S) and skeweness and kurtosis values. When the Kolmogorov-Smirnov is significant ( $\mathrm{p} \leq 0.05)$, it indicates that the data is not normally distributed (Field, 2009). From the findings of K-S test, it can be observed that the null hypothesis was rejected for all the variables except CQ (Seek) and CQ (Share). Thus the assumption of normality is violated for most of the variables. K-S test output in Table 1 and 2 (Appendix 6). However, the K-S test does not provide information about whether the deviation of the distribution from normality is significant enough to bias further analysis (Field, 2009). In large samples ( $>100)$, it is usual to find K-S test significant even when the data only deviates slightly from a normal distribution (Field, 2009). Therefore, as this test is less reliable in large samples (Field, 2009), I examine the skeweness and kurtosis values as well. Table $5.5 \& 5.6$ displays the skeweness, kurtosis, mean, median, standard deviation, minimum and maximum values for all the variables except the controls-Age and Education, as they are dichotomous.

For a sample of more than 300, I look at the absolute value of skewness and kurtosis. A skewness value between -1 and +1 (Hair et al., 2016) and a kurtosis value between -2 and +2 (Pallant, 2010) are considered normal. It is observed that the skewness and kurtosis values are within the thresholds. Although, according to the K-S test the data distribution for the variables were found to be non-normal, but the skewness and kurtosis have been able to satisfy the assumption of normality.

Even if the normality assumption were violated, this is unlikely to have any effect on my data analysis. According to Hayes (2018a) this assumption of normality is rarely met in practice. In large samples normality matters less (Field, 2018, p. 248; Williams, Grajales, \& Kurkiewicz, 2013). Unless the sample size is very small, only the most severe violations of normality assumption affect the validity of statistical inferences from regression analysis (Duncan \& Layard, 1973; Hayes, 1996; Hayes, 2018a). Large samples tend to diminish the detrimental effects of non-normality (Hair et al, 2010, p 71). Moreover, the statistical tool that I use, PROCESS Macro, produces a confidence interval using the bootstrap procedure which does not require the assumption of normality of the distribution (Hayes, 2012). 
Table 5.5 Descriptive statistics- knowledge seeking

\begin{tabular}{cccccccccccc}
\hline & \multicolumn{2}{c}{ N } & Mean & Median & $\begin{array}{c}\text { Std. } \\
\text { Deviation }\end{array}$ & $\begin{array}{c}\text { Skewne } \\
\text { ss }\end{array}$ & $\begin{array}{c}\text { Std. Error } \\
\text { of } \\
\text { Skewness }\end{array}$ & $\begin{array}{c}\text { Kurtosi } \\
\text { s }\end{array}$ & $\begin{array}{c}\text { Std. Error } \\
\text { of } \\
\text { Kurtosis }\end{array}$ & Min & Max \\
& Valid & Missing & & & & & & & & & \\
\hline KSeeking & 333 & 0 & .000 & -.007 & .900 & -.364 & .134 & .024 & .266 & -2.41 & 2.32 \\
Sub Power & 333 & 0 & .000 & -.228 & .881 & -.411 & .134 & .081 & .266 & -2.73 & 2.27 \\
OI & 333 & 0 & .000 & -.014 & .475 & -.533 & .134 & 1.506 & .266 & -2.20 & .88 \\
Comp & 333 & 0 & .000 & -.020 & .537 & .444 & .134 & -.686 & .266 & -.74 & 1.38 \\
CQ & 333 & 0 & .000 & -.006 & .261 & .217 & .134 & .010 & .266 & -.70 & .74 \\
Tenure & 318 & 15 & .000 & -.853 & 4.463 & .508 & .137 & -.469 & .273 & -7.85 & 12.15 \\
Task & 333 & 0 & .000 & -.030 & .765 & -.127 & .134 & -.209 & .266 & -2.03 & 1.97 \\
\hline
\end{tabular}

$\mathrm{K}=$ knowledge; $\mathrm{Sub}=$ Subsidiary; $\mathrm{OI}=$ Organizational identification; Comp=Competition; $\mathrm{CQ}=\mathrm{Cultural}$ intelligence

Table 5.6 Descriptive statistics- knowledge sharing

\begin{tabular}{|c|c|c|c|c|c|c|c|c|c|c|c|}
\hline & Valid & Missing & Mean & Median & $\begin{array}{l}\text { Std. } \\
\text { Deviati } \\
\text { on }\end{array}$ & Skewness & $\begin{array}{c}\text { Std. Error } \\
\text { of } \\
\text { Skewness }\end{array}$ & $\begin{array}{c}\text { Kurtosi } \\
\text { s }\end{array}$ & $\begin{array}{c}\text { Std. Error } \\
\text { of } \\
\text { Kurtosis }\end{array}$ & Min & Max \\
\hline KSharing & 333 & 0 & .000 & .005 & .546 & -.376 & .134 & .670 & .266 & -1.64 & 1.38 \\
\hline Sub Power & 333 & 0 & .000 & .199 & .903 & -.254 & .134 & .182 & .266 & -2.80 & 2.70 \\
\hline OI & 333 & 0 & .000 & -.017 & .476 & -.532 & .134 & 1.505 & .266 & -2.20 & .88 \\
\hline Comp & 333 & 0 & .000 & -.062 & .559 & .489 & .134 & -.630 & .266 & -.87 & 1.44 \\
\hline CQ & 333 & 0 & .000 & -.014 & .272 & .227 & .134 & .150 & .266 & -.73 & .78 \\
\hline Tenure & 318 & 15 & .000 & -.853 & 4.466 & .513 & .137 & -.471 & .273 & -7.85 & 12.15 \\
\hline Task & 327 & 6 & .000 & .080 & 1.083 & .121 & .135 & -.277 & .269 & -1.92 & 2.08 \\
\hline
\end{tabular}

$\mathrm{K}=$ knowledge; Sub=Subsidiary; $\mathrm{OI}=$ Organizational identification; Comp=Competition; $\mathrm{CQ}=\mathrm{Cultural}$ intelligence

\subsubsection{Homoscedasticity}

Homoscedasticity is examined by a visual analysis of the scatter plots for the dependent variables; knowledge seeking and sharing. Also, a Breusch-Pagan and Koenker test (Daryanto, 2013) was used to detect heteroscedasticity. For this test, a significance value less .05 indicates that homoscedasticity is violated (Doh, Bunyaratavej \& Hahn, 2009). For knowledge seeking, it is observed that the variances of the residuals are fairly constant, that is, homoscedasticity is not violated. Also, the Breusch-Pagan and Koenker test results are not significant, indicating that knowledge seeking meets the assumption of homoscedasticity.

But for knowledge sharing, the scatter plot shows that the variances are not equal, that is, heteroscedasticity is present in the relationship between the independent variables and the dependent variable, knowledge sharing. Also, the Breusch-Pagan and Koenker test results are significant. This signals presence of heteroscedasticity for knowledge sharing. According to Hayes and Cai (2007), violation of the homoscedasticity assumption can affect the validity and power of statistical tests when using OLS regression. So they suggest the use of a 
heteroscedasticity-consistent standard error (HCSE) estimator of OLS parameter estimates (White, 1980; Hayes and Cai, 2007) to reduce the effects of heteroscedasticity. In this approach, although OLS is used to estimate the regression model, the standard errors are estimated using an alternative method which does not assume homoscedasticity. The Process Macro statistical tool that I use for hypotheses testing provides an option for using heteroscedasticity-consistent covariance estimators ( $\mathrm{HC} 3$ or $\mathrm{HC} 4$ ) that don't assume homoscedasticity. Therefore, while analysing the knowledge sharing model, I use a heteroscedasticity consistent standard error estimator by choosing the option HC3 in PROCESS. The scatter plot output for seeking and sharing are in Figure $1 \& 2$ (Appendix 7) respectively, the Breusch-Pagan and Koenker test results for seeking and sharing are presented in Table $1 \& 2$ (Appendix 7) respectively.

\subsubsection{Non-independence of errors}

This assumption is tested with intra-class correlations (ICC), which is the variance attributable to group membership (Bliese, 1998; Raudenbush \& Bryk, 2002). A high ICC indicates a higher similarity between members within the same R\&D and a higher difference between groups. A high ICC would lead to violation of the assumption of independence of observations as the observations within the same group will then depend on some group characteristics. A oneway random effects ANOVA is conducted on the perception-based variables based on 40 group memberships and average group size of 8.32.

The ICC value for subsidiary power with respect to R\&Ds is 0.05 , which means $5 \%$ of the variance is explained by variation across R\&Ds. A low ICC value means that there is less agreement within the group. The ICC values in this study are quite low, between 0.03 to 0.19 (from Table 5.7), which implies that individual level of analysis is appropriate for this study (Smidts, Pruyn, \& van Riel, 2001). Also, Bliese (2000) recommends that ICC values above 0.20 indicate a desirable level for aggregation of group effects (Hon \& Lu, 2015). As observed, the ICC values here fall short of this benchmark. Therefore, it can be considered that the observations are sufficiently independent. 
Table 5.7 Intra-class correlations

\begin{tabular}{lc}
\hline \multicolumn{1}{c}{ Variables } & ICC \\
\hline SPSeek & 0.056 \\
SPShare & 0.039 \\
KSeek & 0.072 \\
KShare & 0.066 \\
OI & 0.199 \\
CompSeek & 0.151 \\
CompShare & 0.156 \\
CQ & 0.187 \\
Task & 0.097 \\
\hline gence &
\end{tabular}

\subsubsection{Multicollinearity}

Pearson's correlation coefficient, Variance Inflation Factor (VIF) and Tolerance (T) have been examined to investigate the relationship strength between the independent variables, subsidiary power, identification, intra-MNE competition, cultural intelligence, age, tenure, education and task interdependence, as well as with the dependent variables; knowledge seeking and sharing. Pearson's correlation coefficient values between the variables have been found to be less than the threshold of 0.5 (Hair, Anderson, Tatham, \& Black, 1995), which indicates an absence of multicollinearity.

VIF values for all variables are found to be less than the threshold of 10 (Myers, 1990; Field, 2018), ranging from 0.806 to 0.983. Also, the T value is greater than 0.1 (Myers, 1990; Field, 2018), ranging from 1.017 to 1.240 . This indicates that there is no multicollinearity problem in my data. The correlations, VIF and $\mathrm{T}$ output is presented in Table 5.8 and 5.9 for knowledge seeking and sharing respectively.

\subsection{Hypothesis Testing}

\subsubsection{Direct and mediation effect}

To test the direct and indirect effect of subsidiary power on interpersonal knowledge transfer, I have used Process Model 4 which is the mediation model. The results are presented in Figure 5.2 and Table 5.10.

H1a argued for the direct negative effect of subsidiary power on knowledge seeking, while $\mathrm{H} 1 \mathrm{~b}$ asserted on the direct positive effect on knowledge sharing. I found support for $\mathrm{H} 1 \mathrm{~b}$ but not for H1a. While the effect of subsidiary power on knowledge sharing was found to be positive and significant, $\mathrm{C}^{\prime}=0.103, \mathrm{p}<.01$ as per the hypothesis; the effect on knowledge seeking was found to be positive and non-significant, $C^{\prime}=0.058, p=0.317$, against the hypothesis. This indicates that the power of a subsidiary can positively influence individual 
Table 5.8 Correlations output-Knowledge Seeking

\begin{tabular}{|c|c|c|c|c|c|c|c|c|c|c|c|c|c|}
\hline \multirow[b]{2}{*}{ Variables } & \multicolumn{11}{|c|}{ Knowledge Seeking } & \multicolumn{2}{|c|}{ Collinearity Statistics } \\
\hline & Mean & $\begin{array}{c}\text { Std. } \\
\text { Deviation }\end{array}$ & $\begin{array}{l}\text { Knowledge } \\
\text { Seeking }\end{array}$ & $\begin{array}{l}\text { Subsidiary } \\
\text { Power }\end{array}$ & $\begin{array}{l}\text { Identifi } \\
\text { cation }\end{array}$ & $\begin{array}{l}\text { Competiti } \\
\text { on }\end{array}$ & $\begin{array}{c}\text { Cultural } \\
\text { Intelligen } \\
\text { ce }\end{array}$ & $\begin{array}{c}\text { Relative } \\
\text { Age }\end{array}$ & Tenure & Education & $\begin{array}{c}\text { Task } \\
\text { interdepend } \\
\text { ence }\end{array}$ & Tolerance & VIF \\
\hline Knowledge Seeking & .000 & .900 & 0.914 & & & & & & & & & \multicolumn{2}{|c|}{ Dependent variable } \\
\hline Subsidiary Power & .000 & .881 & .089 & 1 & & & & & & & & .947 & 1.056 \\
\hline Identification & .000 & .475 & $.157 * *$ & $.129 *$ & 0.743 & & & & & & & .806 & 1.240 \\
\hline Competition & .000 & .537 & .082 & $.120 *$ & -.044 & 0.713 & & & & & & .971 & 1.029 \\
\hline Cultural Intelligence & .000 & .261 & $.331 * *$ & .084 & $.303 * *$ & -.078 & 0.863 & & & & & .886 & 1.129 \\
\hline Age & .000 & .499 & -.093 & .043 & .032 & -.016 & .043 & 1 & & & & .983 & 1.017 \\
\hline Tenure & .000 & 4.463 & $.134^{*}$ & .005 & $.265^{* *}$ & .030 & -.014 & .038 & 1 & & & .905 & 1.105 \\
\hline Education & .000 & .497 & -.064 & -.045 & $.151 * *$ & .029 & .022 & .086 & .105 & 1 & & .951 & 1.051 \\
\hline Task interdependence & -.003 & .765 & .058 & -.054 & .055 & .021 & -.082 & -.039 & $.137 *$ & .003 & 1 & .966 & 1.036 \\
\hline
\end{tabular}

Table 5.9 Correlations output-Knowledge Sharing

\begin{tabular}{|c|c|c|c|c|c|c|c|c|c|c|c|c|c|}
\hline \multirow[b]{2}{*}{ Variables } & \multicolumn{11}{|c|}{ Knowledge Sharing } & \multicolumn{2}{|c|}{ Collinearity Statistics } \\
\hline & Mean & $\begin{array}{c}\text { Std. } \\
\text { Deviation }\end{array}$ & $\begin{array}{c}\text { Knowledge } \\
\text { Sharing }\end{array}$ & $\begin{array}{c}\text { Subsidiary } \\
\text { Power }\end{array}$ & $\begin{array}{l}\text { Identifica } \\
\text { tion }\end{array}$ & $\begin{array}{l}\text { Competiti } \\
\text { on }\end{array}$ & $\begin{array}{c}\text { Cultural } \\
\text { Intelligen } \\
\text { ce }\end{array}$ & $\begin{array}{l}\text { Relativ } \\
\text { e Age }\end{array}$ & Tenure & Education & $\begin{array}{c}\text { Task } \\
\text { Interdepend } \\
\text { ence }\end{array}$ & Tolerance & $\begin{array}{l}\text { VIF } \\
\end{array}$ \\
\hline Knowledge Sharing & .000 & .546 & 0.934 & & & & & & & & & \multicolumn{2}{|c|}{ Dependent variable } \\
\hline Subsidiary Power & .000 & .903 & $.176^{* *}$ & 1 & & & & & & & & .946 & 1.057 \\
\hline Identification & .000 & .476 & $.112 *$ & $.127 *$ & 0.743 & & & & & & & .816 & 1.226 \\
\hline Competition & .000 & .559 & $.335 * *$ & .090 & -.041 & 0.718 & & & & & & .966 & 1.035 \\
\hline Cultural Intelligence & .000 & .272 & $.405 * *$ & $.138 *$ & $.308 * *$ & -.064 & 0.865 & & & & & .869 & 1.151 \\
\hline Age & .000 & .494 & -.011 & .062 & .085 & -.092 & .091 & 1 & & & & .971 & 1.030 \\
\hline Tenure & .000 & 4.466 & .071 & -.010 & $.264 * *$ & .042 & -.013 & .026 & 1 & & & .917 & 1.091 \\
\hline Education & .000 & .497 & -.018 & -.045 & $.152 * *$ & .008 & .025 & .023 & .103 & 1 & & .964 & 1.037 \\
\hline Task interdependence & -.002 & 1.083 & $-.161 * *$ & .047 & -.062 & .032 & .097 & .049 & -.010 & .017 & 1 & .970 & 1.031 \\
\hline
\end{tabular}

** Correlation is significant at the 0.01 level (2-tailed)

* Correlation is significant at the 0.05 level (2-tailed).

Diagonal values in bold: $\mathrm{Sq}$ root of AVE 
knowledge sharing. The higher the relative power, the commensurate sharing of knowledge will be more, or the lower the relative power, sharing will be less. With respect to knowledge seeking, there is no evidence of a direct relationship with subsidiary power.

$\mathrm{H} 2 \mathrm{a}$ and $\mathrm{H} 2 \mathrm{~b}$ hypothesized a mediation effect of organizational identification in the relationships between subsidiary power and knowledge seeking/sharing. In addition, $\mathrm{H} 3 \mathrm{a}$ and $\mathrm{H} 3 \mathrm{~b}$ hypothesized the direction of these mediation relationships. While the indirect effect of subsidiary power on knowledge seeking is hypothesized as negative, on knowledge sharing it is hypothesized as positive.

An output is produced for the indirect mediation effect with a $95 \%$ bootstrap confidence interval using 5000 bootstrap samples. As per the test, evidence of an indirect mediation effect was found for the relationship of subsidiary power and knowledge seeking through organizational identification, 0.023 with bootstrap confidence interval: 0.003 to 0.054 .

The confidence interval does not contain zero, which proves that organizational identification mediates the relationship. Subsidiary power impacts employees' identification with the subsidiary which influences their knowledge seeking behaviour. Thus, $\mathrm{H} 2 \mathrm{a}$ is supported. Although a single inference about the indirect mediation effect is quantified with ab $=0.022$, we should not ignore ' $a$ ' and ' $b$ ' while interpreting the results (Hayes \& Rockwood, 2017). As predicted, 'a', the effect of subsidiary power on identification is positive. But, against prediction, ' $b$ ', the effect of OI on knowledge seeking was found to be positive rather than negative. Therefore, against $\mathrm{H} 3 \mathrm{a}$, it was found that the mediation effect is positive $(0.022)$ rather than negative. This means that when employees perceive that the relative subsidiary power is high, they identify more with the subsidiary which in turn leads to higher knowledge seeking. Thus, H3a is not supported as a positive mediation effect is found.

As per the mediation output, there is no evidence of an indirect mediation effect of subsidiary power on knowledge sharing through organizational identification, 0.005 , as the bootstrap confidence interval, -0.003 to 0.01 , contains zero. Although 'a', the effect of subsidiary power on identification is positive and significant, ' $b$ ' the effect of identification on knowledge sharing was found to be non-significant. This means, I did not find evidence for the influence of subsidiary power on employees' knowledge sharing through their identification with the subsidiary. Thus, $\mathrm{H} 2 \mathrm{~b}$ is not supported. As per $\mathrm{H} 3 \mathrm{~b}$, the effect is found to be positive. But there is no evidence of mediation. Therefore, H3b is also not supported. 
Figure 5.2 Mediation effects

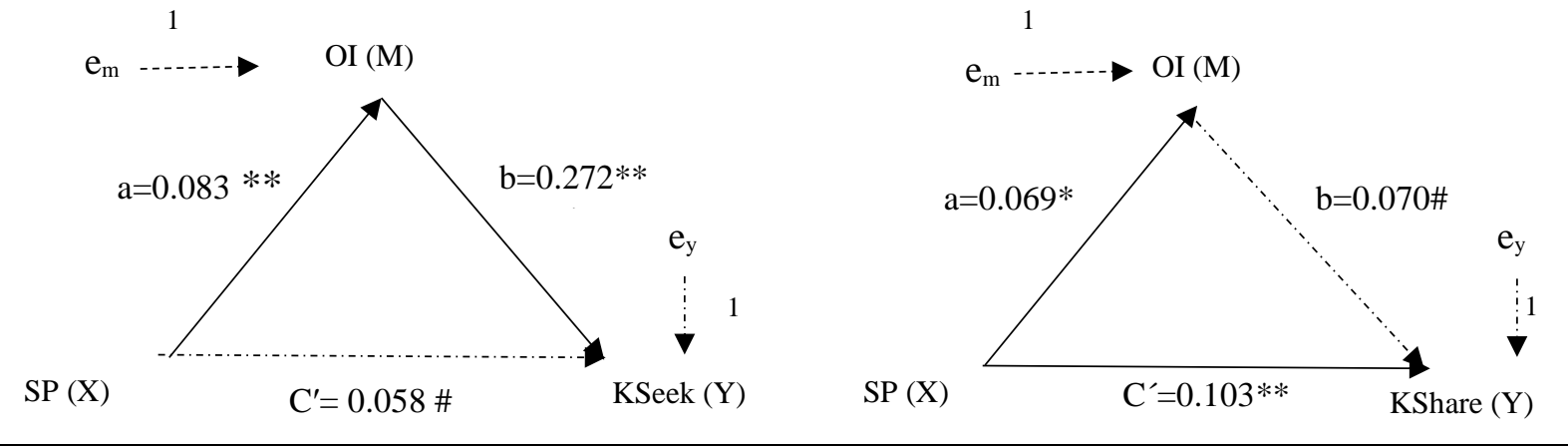

Table 5.10 Mediation effects

\begin{tabular}{|c|c|c|c|c|c|}
\hline Variables & OI & KSeek & \multicolumn{2}{|c|}{ OI } & KShare \\
\hline Constant & $-.272 *$ & -.014 & \multicolumn{2}{|c|}{-.145} & $.306 * *$ \\
\hline Age & .006 & $-.184^{\mp}$ & \multicolumn{2}{|c|}{.062} & -.011 \\
\hline Tenure & $.025^{* * * *}$ & .019 & \multicolumn{2}{|c|}{$.025^{* * *}$} & .008 \\
\hline Education & $.137 * *$ & -.146 & \multicolumn{2}{|c|}{$.119^{*}$} & -.025 \\
\hline Task dependence & .020 & .031 & \multicolumn{2}{|c|}{-.032} & $-.087 * *$ \\
\hline Sub Power $(X)$ & $.083 * *$ & .058 & \multicolumn{2}{|c|}{$.069^{*}$} & $.103 * *$ \\
\hline$O I(M)$ & & $.272 * *$ & & & .070 \\
\hline$R^{2}$ & .106 & .055 & \multicolumn{2}{|c|}{.102} & .077 \\
\hline$F$ & $7.197 * * *$ & $2.967^{* *}$ & \multicolumn{2}{|c|}{$5.805 * * *$} & $3.349^{* *}$ \\
\hline \multicolumn{3}{|c|}{ Mediation by $\mathrm{OI}$} & \multicolumn{3}{|c|}{ Mediation by $\mathrm{OI}$} \\
\hline Mediation Effect & BootLLCI & BootULCI & Mediation Effect & BootLLCI & BootULCI \\
\hline .022 & .003 & .054 & .005 & -.003 & .019 \\
\hline Direct Effect & LLCI & ULCI & Direct Effect & LLCI & ULCI \\
\hline .058 & -.056 & .172 & .103 & .018 & .188 \\
\hline
\end{tabular}

Table 5.11 Effect sizes

\begin{tabular}{cccccccc}
\hline Indirect Effect & Effect Size & BootLLCI & BootULCI & Direct Effect & Effect Size & LLCI & ULCI \\
\hline $\begin{array}{c}\text { Partially } \\
\text { standardized }\end{array}$ & .025 & .003 & .059 & $\begin{array}{c}\text { Partially } \\
\text { standardized }\end{array}$ & .195 & .018 & .188 \\
$\begin{array}{c}\text { Completely } \\
\text { standardized }\end{array}$ & .022 & .003 & .052 & $\begin{array}{c}\text { Completely } \\
\text { standardized }\end{array}$ & .174 & .018 & .188 \\
\hline
\end{tabular}

${ }^{\mp} \mathrm{p}<.10 * \mathrm{p}<.05 * * \mathrm{p}<.01 * * * \mathrm{p}<.001$ \#Non-significant. Dotted lines in the figure represent non-significant relationship; $\mathrm{SP}=$ Subsidiary Power; OI=Organizational Identification; KSeek=Knowledge Seeking; KShare=Knowledge Sharing $\mathrm{e}_{\mathrm{m}}$ \& $e_{y}=$ errors in the estimation of $\mathrm{M}(\mathrm{OI})$ and $\mathrm{Y}(\mathrm{KSeek} / \mathrm{Share})$ respectively; $\mathrm{R}^{2}=$ Coefficient of determination; $\mathrm{F}=\mathrm{F}$ statistic 
Table 5.11 presents the Effect Sizes of the indirect mediation effect for knowledge seeking and direct effect for knowledge sharing. The partially standardized $\left(a b_{p s}\right)$ and completely standardized $\left(a b_{c s}\right)$ effect sizes for the indirect effect in the knowledge seeking model is found to be small (Cohen, 1988), 0.025 and 0.022 respectively. The average effect size in international business research is small (Ellis, 2010). For the knowledge sharing model, the partially standardized ( $c^{\prime}$ ps) and completely standardized ( $c^{\prime}$ cs) effect sizes for the direct effect are found to be greater than medium (Cohen, 1988), 0.195 and 0.174 respectively.

\subsubsection{Moderation effect of competition and cultural intelligence}

Moderation by competition and cultural intelligence on the indirect effect is argued to take place in the second stage, on the effect of organizational identification on knowledge seeking/sharing. Therefore, before examining the moderation on the indirect effect, I investigate and show moderation of competition and cultural intelligence on the direct effect of organizational identification on knowledge seeking and sharing. For this purpose, I use Process Model 1 for each of the moderators. The moderation effect of intra-MNE competition and cultural intelligence on the direct effect of organizational identification on knowledge seeking and sharing is presented in Figure 5.3.

It is found that intra-MNE competition moderates the effect of organizational identification on knowledge sharing and not on knowledge seeking (Figure 5.3a). The effect on knowledge seeking is non-significant. But the effect on knowledge sharing is negative and significant, $-0.204, p=0.05(<0.05)$. This means, the effect of OI on knowledge sharing will vary with variation in intra-MNE competition. Two employees having similar identification with the subsidiary, will have different levels of knowledge sharing if their perceived levels of intra-MNE competition are different. With higher levels of intra-MNE competition, knowledge sharing will be less. The visual depiction of the moderating effect is in Figure 5.3c (ii).

The moderation by cultural intelligence is presented in Figure 5.3b. It is found that cultural intelligence moderates the direct effect of organizational identification on knowledge seeking, but doesn't moderate the effect on knowledge sharing. The effect on knowledge sharing is non-significant. The moderation effect on knowledge seeking is positive and significant, $0.889 \mathrm{p}=0.02$. This means, the effect of OI on knowledge seeking will vary with variation in cultural intelligence. Two employees having similar levels of identification with the subsidiary will have different levels of knowledge seeking if their cultural intelligence is different. With higher cultural intelligence, knowledge seeking will be more. The visual depiction of this moderating effect is in Figure 5.3c (i). 
Figure 5.3 Moderation of OI on knowledge seeking and sharing by competition and cultural intelligence

Knowledge Seeking Knowledge Sharing

a. Moderation on the direct effect by competition
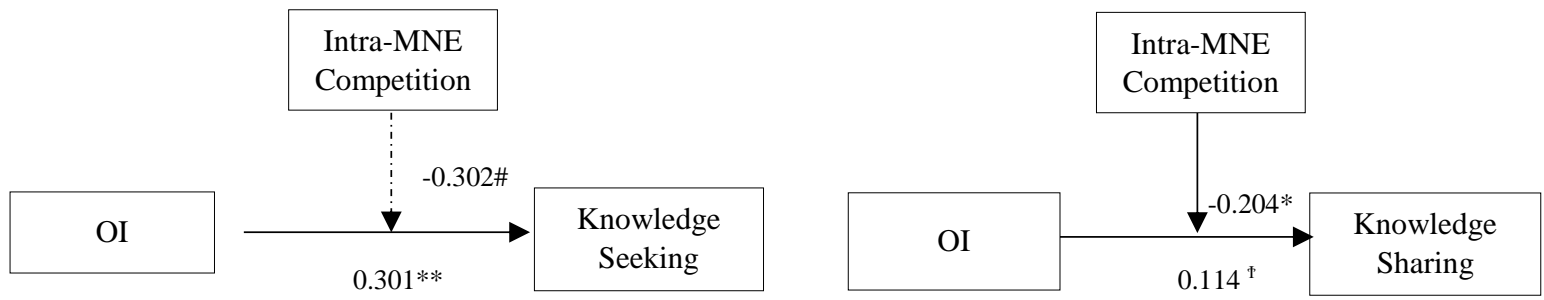

b. Moderation on the direct effect by $C Q$

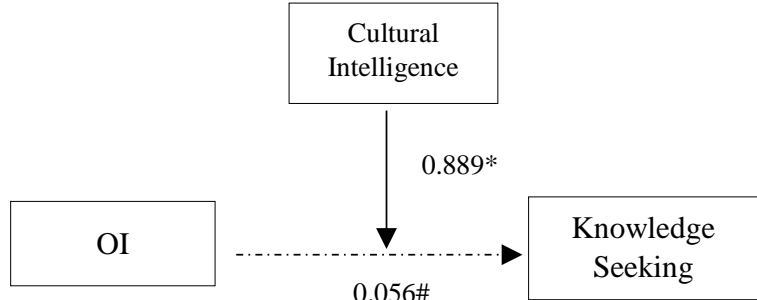

c. (i) Moderation effect of $C Q$

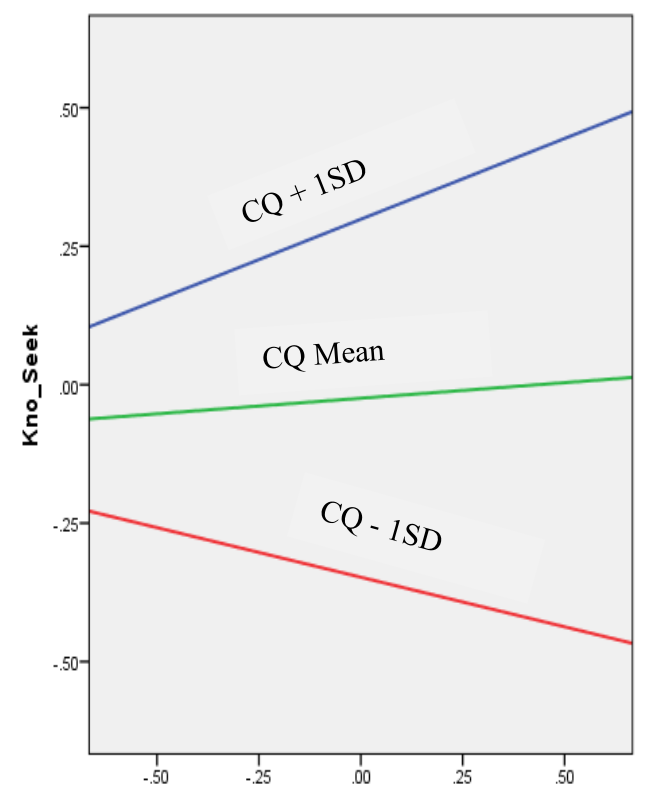

$\mathrm{OI}+1 \mathrm{SD}$ and $-1 \mathrm{SD}$

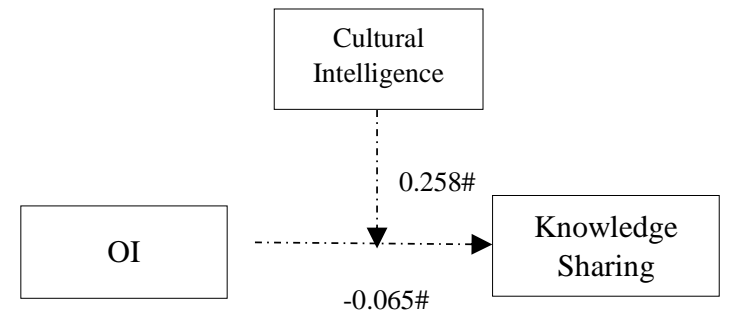

(ii) Moderation effect of competition

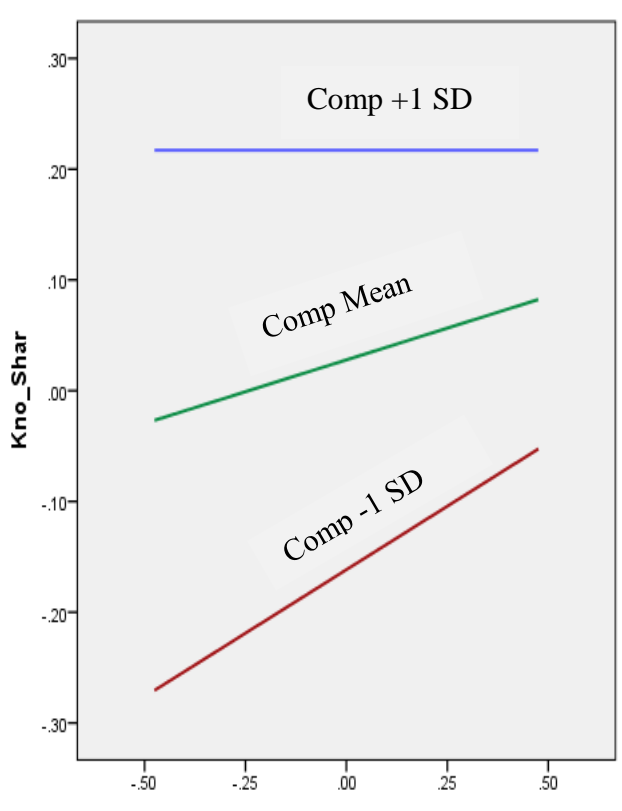

$\mathrm{OI}+1 \mathrm{SD}$ and $-1 \mathrm{SD}$

$\mathrm{\tau}<.10 * \mathrm{p}<.05 * * \mathrm{p}<.01$ \#Non-significant. Dotted lines in the figure represent non-significant relationship. OI=Organizational identification; $\mathrm{CQ}=$ Cultural intelligence; Comp=Competition; Kno_Seek=knowledge seeking; Kno_Share=knowledge sharing; $\mathrm{SD}=$ Standard deviation 
I test hypotheses H4a, H4b, H5a and H5b using Process Model 16. It is a second stage dual moderated mediation model. These hypotheses argue for the moderation of the indirect effect of subsidiary power on knowledge seeking/sharing through organizational identification by competition and cultural intelligence. The results are presented in Figure 5.4.

Indices of partial moderated mediation with a 95\% bootstrap confidence interval using 5000 bootstrap samples are presented in Table 5.12. From the index of the knowledge seeking model, as hypothesized, I found that the moderation of the indirect mediated effect by cultural intelligence, 0.072 is statistically different from zero (0.001 to 0.168 ), which implies that the indirect effect of subsidiary power on knowledge seeking is associated with the level of cultural intelligence independent of any moderation by intra-MNE competition. Therefore, with $95 \%$ confidence we can say that an employee's cultural intelligence moderates the indirect effect of his/her perception of relative subsidiary power on his/her knowledge seeking through the effect of OI. This means when intra-MNE competition is held constant, the effect of subsidiary power on knowledge seeking varies with changes in an employee's level of CQ. Two employees who have similar perceptions about subsidiary power and the same identification will display different knowledge seeking behaviour if their cultural intelligence is different. The partial moderated mediation effect is positive which means higher levels of cultural intelligence will lead to higher knowledge seeking. Hypothesis H5a was that cultural intelligence will weaken the negative effect of subsidiary power on knowledge seeking. But, as per the finding, subsidiary power has a positive effect on knowledge seeking and cultural intelligence strengthens this effect. Thus, we can conclude that H5a is supported.

I did not find evidence for a moderation effect of intra-MNE competition on the indirect effect of subsidiary power on knowledge seeking. From the index of partial moderated mediation, it is found that although the effect is negative, the bootstrap confidence interval of the effect includes zero (-0.059 to 0.007$)$ which implies that the indirect effect is unrelated to the size of intraMNE competition (independent of any effect of cultural intelligence as CQ is held fixed).

Therefore, at the level of $95 \%$ confidence we can rule out that competition is related to the indirect effect of subsidiary power on knowledge seeking through organizational identification. That is, independent of the effect of cultural intelligence on the indirect effect, the evidence doesn't support the claim that the indirect effect differs between two different levels of competition. Also, although the index is negative, it is non-significant. So, hypothesis H4a is not supported. 
From the index of partial moderated mediation in the knowledge sharing model, as hypothesized, I found that the moderation of the indirect mediated effect by intra-MNE competition, -0.014 is statistically different from zero (-0.0392 to -0.0002) (from Table 5.12), which implies that the indirect effect of subsidiary power on knowledge sharing is associated with the level of intra-MNE competition independent of any moderation by cultural intelligence. Therefore, with 95\% confidence we can say that an employee's perceived intra-MNE competition moderates the indirect effect of his/her perception of relative subsidiary power on his/her knowledge sharing through the effect of OI. This means when cultural intelligence is held constant, the effect of subsidiary power on knowledge sharing varies with changes in an employee's level of perceived intra-MNE competition. Two employees who have similar perceptions about subsidiary power and the same identification will display different knowledge sharing behaviour if their perception about intraMNE competition is different. The partially moderated mediation effect is negative which means the effect of subsidiary power on knowledge sharing will be weakened by competition, that is, with higher competition levels there will be lesser knowledge sharing. Thus, hypothesis H4b is supported.

I did not find evidence for a moderation effect of CQ on the indirect effect of subsidiary power on knowledge sharing. From the index of partial moderated mediation, it is found that the bootstrap confidence interval of the effect includes zero (-0.019 to 0.060$)$ which implies that the indirect effect is unrelated to the size of cultural intelligence (independent of any effect of intraMNE competition, as competition is held fixed).

Therefore, at the level of $95 \%$ confidence we can rule out that CQ is related to the indirect effect of subsidiary power on knowledge sharing through organizational identification. That is, independent of the effect of competition on the indirect effect, the evidence doesn't support the claim that the indirect effect differs between two different levels of CQ. So, hypothesis H5b is not supported. 

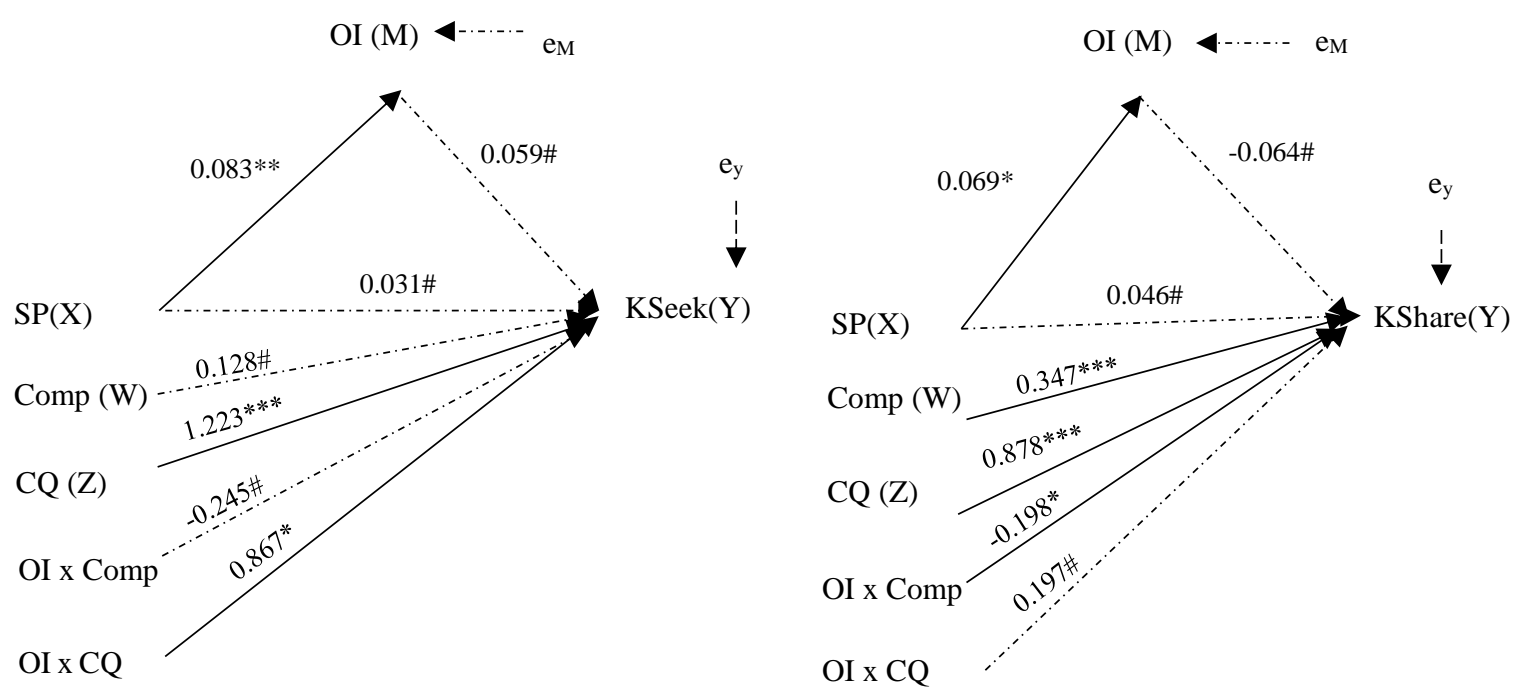

$* \mathrm{p}<.05 \quad * * \mathrm{p}<.01 * * * \mathrm{p}<.001$ \#Non-significant. Dotted lines indicate non-significant relationships. SP=Subsidiary power; $\mathrm{OI}=$ Organizational identification; KSeek=Knowledge seeking; KShare=Knowledge sharing; Comp=Competition; $\mathrm{CQ}=\mathrm{Cultural}$

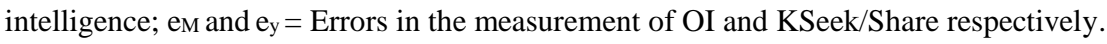

Table 5.12 Indices of partial moderated mediation

\begin{tabular}{ccccccccc}
\hline & \multicolumn{4}{c}{ Knowledge Seeking } & \multicolumn{3}{c}{ Knowledge Sharing } \\
\hline & Index & BootSE & BootLLCI & BootULCI & Index & BootSE & BootLLCI & BootULCI \\
\hline Comp & -.020 & .043 & -.059 & .007 & -.014 & .010 & -.039 & -.0002 \\
CQ & .072 & .016 & .001 & .168 & .014 & .019 & -.019 & .060 \\
\hline
\end{tabular}

Comp=Competition; $\mathrm{CQ}=$ Cultural intelligence

\subsubsection{Probing moderation}

I further explored the conditional effect of cultural intelligence to probe the level at which the moderation is significant. Taking $-1 \mathrm{SD}$, Mean, and $+1 \mathrm{SD}$ as low, moderate and high levels of CQ respectively, and holding intra-MNE competition constant at $-1 \mathrm{SD}$, mean and $+1 \mathrm{SD}$, I probed the interaction, which is presented in Table 5.13. From the analysis it was found that at $95 \%$ bootstrap CIs for indirect effects involving those with higher levels of CQ (+1SD) when competition is held constant at lower or moderate levels, did not include zero (.004 to $.088 \& .001$ to .069$)$ in bold in Table 5.13), indicating meaningful indirect effects. This suggests that the indirect effect of relative subsidiary power varies depending on one's level of CQ, making the subsidiary power ineffective 
for those with medium and low CQ, but most effective for those with high CQ while competition is either at low or moderate levels (Dixon, 2016).

Table 5.13 Probing moderated mediation by cultural intelligence in the knowledge seeking model.

\begin{tabular}{cccccc}
\hline CQ & Comp & Effect & BootSE & BootLLCI & BootULCI \\
\hline -1SD & -1SD & -.003 & .016 & -.034 & .032 \\
Mean & -1 SD & .017 & .015 & -.005 & .053 \\
$+1 \mathrm{SD}$ & $-1 \mathrm{SD}$ & .035 & .022 & $\mathbf{. 0 0 5}$ & $\mathbf{. 0 8 8}$ \\
& & & & & \\
$-1 \mathrm{SD}$ & Mean & -.014 & .013 & -.041 & .012 \\
Mean & Mean & .006 & .009 & -.008 & .029 \\
$+1 \mathrm{SD}$ & Mean & .027 & .017 & $\mathbf{. 0 0 1}$ & $\mathbf{. 0 6 9}$ \\
& & & & & \\
$-1 \mathrm{SD}$ & $+1 \mathrm{SD}$ & -.025 & .015 & -.057 & .003 \\
Mean & $+1 \mathrm{SD}$ & -.004 & .011 & -.025 & .020 \\
$+1 \mathrm{SD}$ & $+1 \mathrm{SD}$ & .016 & .017 & -.011 & .056 \\
\hline
\end{tabular}

The interaction effects can be visualized in Figure 5.5. It shows that the effect of an employee's organizational identification on knowledge seeking is different at different values of CQ -1SD, Mean and $+1 \mathrm{SD}$, depicted by the three different coloured lines. Moderation by CQ is shown at three different levels of intra-MNE competition (holding intra-MNE competition constant).

Therefore, it can be concluded that independent of any moderation of the indirect effect of subsidiary power by intra-MNE competition, cultural intelligence positively moderates this indirect effect. This is an inference about the slope of the lines in the Figure 5.6. But, independent of the effect of cultural intelligence on the indirect effect, the evidence does not support a claim that the indirect effect differs for different levels of intra-MNE competition. This is an inference about the gap between the lines in the Figure 5.6 (Hayes, 2018b). 
Figure 5.5 Interaction effect of CQ keeping competition constant at low, moderate and high levels

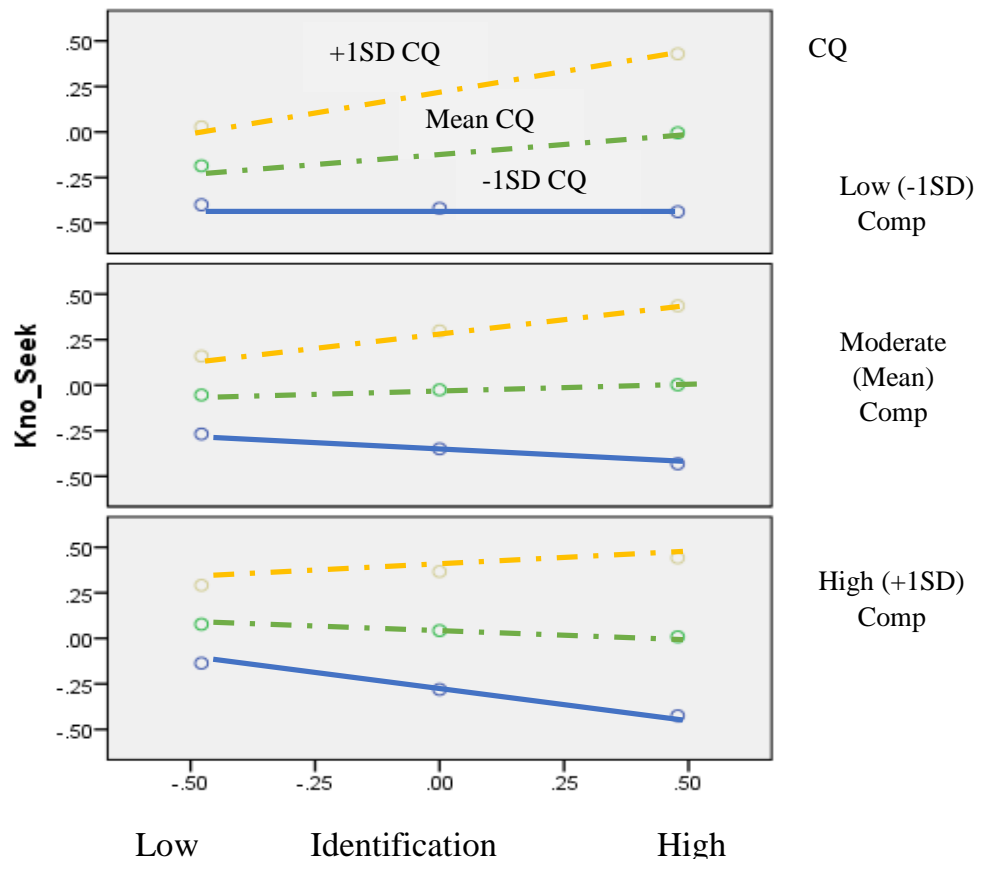

Figure 5.6 Moderation by $C Q$ on the indirect effect

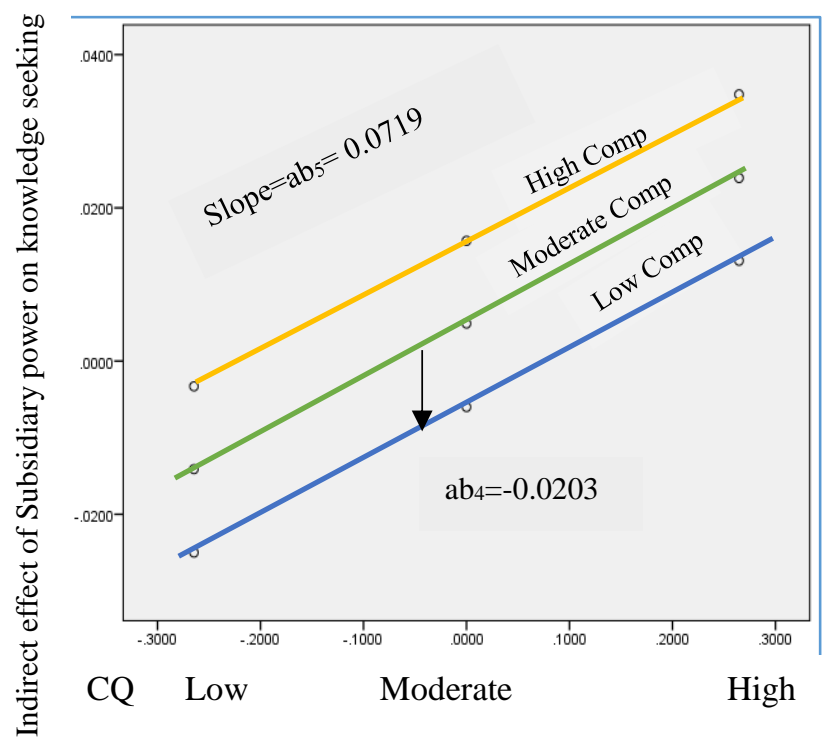


Further, I probed the partial moderated mediation of competition on the indirect effect of subsidiary power on knowledge sharing, as competition was found to moderate the indirect effect, to find the level at which moderation is significant. Similar to the knowledge seeking model, I probed the partial moderated mediation considering low (-1SD), moderate (mean) and high $(+1 \mathrm{SD})$ levels of Competition. Table 5.14 shows estimates with bootstrap confidence intervals of the conditional effect of subsidiary power on knowledge sharing at various combinations of competition and cultural intelligence (CQ held constant). It is found that at $95 \%$ bootstrap confidence intervals for the indirect effects involving competition at the higher $(+1 \mathrm{SD})$ levels (while CQ is held constant either at lower or moderate levels) doesn't include zero (-.040 to -.001; -.031 to -.001), implying meaningful indirect effects. This indicates that the indirect effect of relative subsidiary power on knowledge sharing varies depending on the level of perceived intraMNE competition, making the effect of subsidiary power ineffective for competition at lower and moderate levels, but most effective for those perceiving higher intra-MNE competition when CQ is at lower or moderate levels (Dixon, 2016).

Table 5.14 Probing moderated mediation by intra-MNE competition in the knowledge sharing model.

\begin{tabular}{cccccc}
\hline Comp & CQ & Effect & BootSE & BootLLCI & BootULCI \\
\hline -1SD & -1SD & -.001 & .007 & -.015 & .017 \\
Mean & -1SD & -.008 & .007 & -.024 & .003 \\
+1 SD & -1SD & -.016 & .010 & $\mathbf{- . 0 4 0}$ & $\mathbf{- . 0 0 1}$ \\
& & & & & \\
-1SD & Mean & .003 & .006 & -.006 & .019 \\
Mean & Mean & -.004 & .004 & -.015 & .003 \\
+1 SD & Mean & -.012 & .008 & $\mathbf{- . 0 3 1}$ & $\mathbf{- . 0 0 1}$ \\
& & & & & .029 \\
-1SD & +1 SD & .007 & .009 & -.006 & .014 \\
Mean & +1 SD & -.001 & .007 & -.015 & .006 \\
+1 SD & $+1 S D$ & -.008 & .009 & -.029 & \\
\hline
\end{tabular}

The interaction effects can be visualized in Figure 5.7. It shows that the effect of an employee's organizational identification on knowledge sharing is different at different values of Comp -1SD, Mean and +1 SD, depicted by the three different coloured lines. Moderation by Comp is shown at three different levels of CQ by holding CQ constant.

Therefore, it can be concluded that independent of any moderation of the indirect effect of subsidiary power by $\mathrm{CQ}$, competition negatively moderates this indirect effect. This is an inference about the slope of the lines in Figure 5.8. But, independent of the effect of competition on the 
indirect effect, the evidence does not support a claim that the indirect effect differs for different levels of CQ. This is an inference about the gap between the lines in Figure 5.8 (Hayes, 2018b).

Figure 5.7 Interaction effect of competition keeping CQ constant at low, moderate and high levels

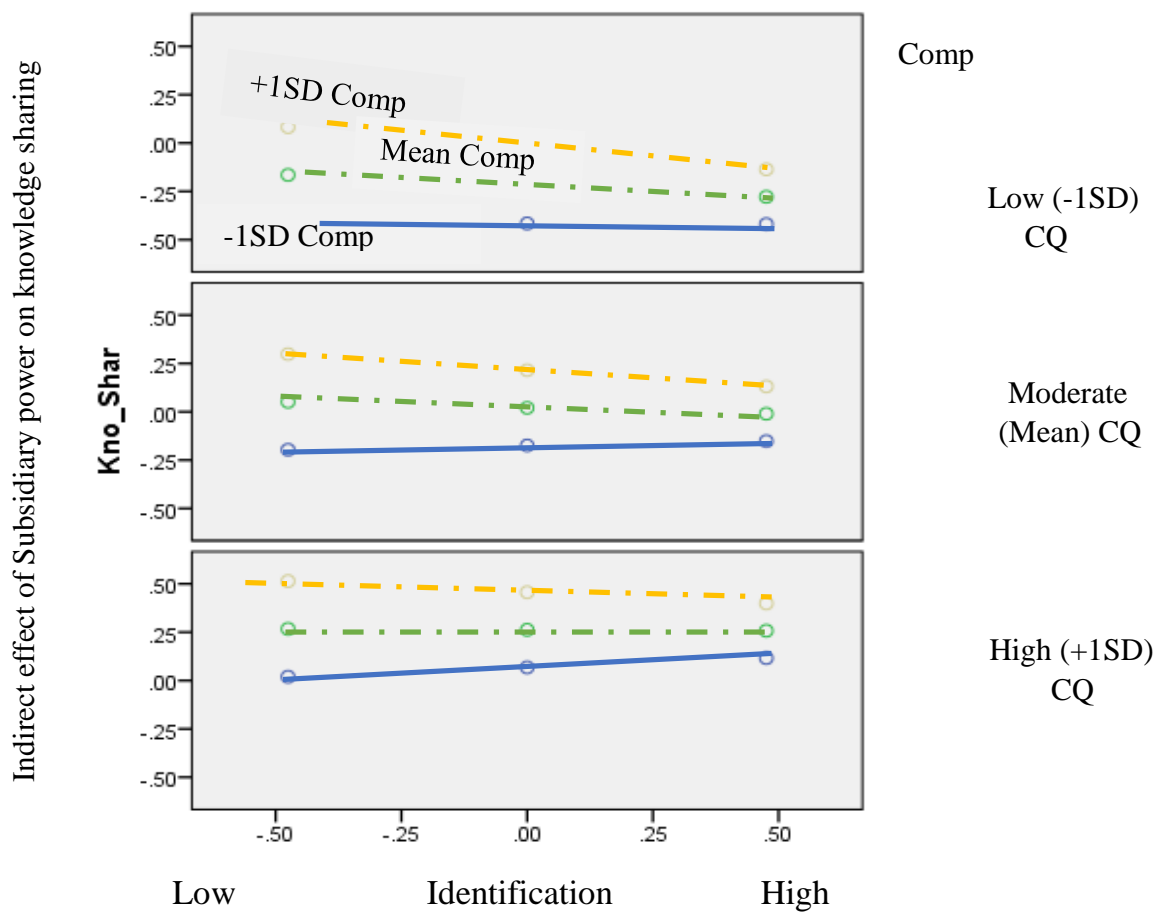

Figure 5.8 Moderation by competition on the indirect effect

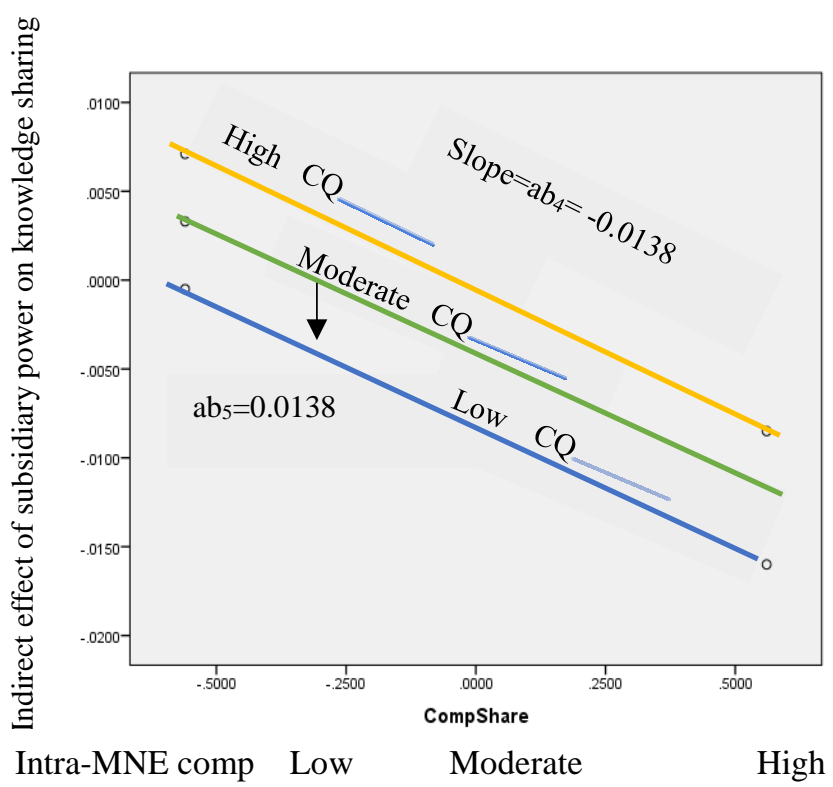


The results for all the models analysed are compiled in Table 5.15 for knowledge seeking and Table 5.16 for knowledge sharing. Table 5.17 presents a summary of all hypotheses tests findings. The $\mathrm{R}^{2}$ for knowledge seeking model is 0.19 , which indicates that $19 \%$ of the variation in knowledge seeking is explained by the variables. Also, it is found that the $\mathrm{R}^{2}$ value by regressing the control variables alone on knowledge seeking is .03, which indicates that the control variables account for about $3 \%$ of the variance in knowledge seeking. Therefore, it can be inferred that all the variables excluding the control variables account for approximately $16 \%$ of the variance in knowledge seeking.

Similarly, in the knowledge sharing model, the $\mathrm{R}^{2}$ value is 0.37 , which indicates that the variables explain $37 \%$ of the variation in knowledge sharing. By regressing the control variables on sharing it is found that the $\mathrm{R}^{2}$ is .04 . Therefore, the variables excluding the controls account for about $34 \%$ of the variance in knowledge sharing.

Among the control variables, age and tenure are found to have significant effects on knowledge seeking, -0.20 and .023 respectively. The relative age of the respondent is negatively related to seeking, -.20. Age was coded as ' 0 ' when a respondent is younger and ' 1 ' when respondent is equal or older to the contact. This indicates that when the respondent is equal or older than the contact, knowledge seeking is less. The positive relationship of seeking with tenure of the respondent implies that knowledge seeking is greater when the employee's tenure in the organization is greater. In the knowledge sharing model, tenure and task dependence are both found to have negative significant effects on knowledge sharing, -.013 and -.127 respectively. This means, the greater the tenure of the respondent working in the organization, the lesser is the knowledge sharing. Also, the higher the task interdependence of the contact on the respondent, the lesser is the knowledge sharing by the respondent is less. 
Table 5.15 Summary results- knowledge seeking

\begin{tabular}{|c|c|c|c|c|c|c|}
\hline & \multicolumn{2}{|c|}{ Mediation model } & \multirow{2}{*}{$\begin{array}{c}\begin{array}{c}\text { Moderation effect of } \\
\text { Comp }\end{array} \\
\text { Kseek }\end{array}$} & \multirow{2}{*}{$\begin{array}{c}\text { Moderation effect of } \\
\text { CQ } \\
\text { Kseek }\end{array}$} & \multicolumn{2}{|c|}{$\begin{array}{l}\text { Moderated mediation } \\
\text { (full model) }\end{array}$} \\
\hline & $\mathrm{OI}$ & Kseek & & & $\mathrm{OI}$ & Kseek \\
\hline Constant & $-.272 *$ & -.014 & -.054 & -.298 & $-.272 *$ & -.289 \\
\hline Age & .006 & $-.185^{\mathrm{T}}$ & $-.169^{\mathrm{T}}$ & $-.207 *$ & .006 & $-.199 *$ \\
\hline Tenure & $.025 * * *$ & .019 & .018 & $.030 * *$ & $.025 * * *$ & $.029 * *$ \\
\hline Education & $.137 * *$ & -.146 & -.152 & -.156 & $.137 * *$ & -.153 \\
\hline Task & .020 & .031 & .029 & .078 & .020 & .082 \\
\hline $\mathrm{SP}(\mathrm{X})$ & $.083 * *$ & .058 & & & $.083 * *$ & .031 \\
\hline $\mathrm{OI}(\mathrm{M})$ & & $.272 * *$ & $.301 * *$ & .056 & & .059 \\
\hline Comp & & & .108 & & & .128 \\
\hline $\mathrm{CQ}$ & & & & $1.221 * * *$ & & $1.223 * * *$ \\
\hline OI x Comp & & & -.302 & & & -.245 \\
\hline OI x CQ & & & & $.889 *$ & & $.867 *$ \\
\hline $\mathrm{R}^{2}$ & .106 & .055 & .061 & .187 & .106 & .197 \\
\hline $\mathrm{F}$ & $7.197 * * *$ & $2.967 * *$ & $2.835^{* *}$ & $9.939 * * *$ & $7.197 * *$ & $7.352 * *$ \\
\hline
\end{tabular}

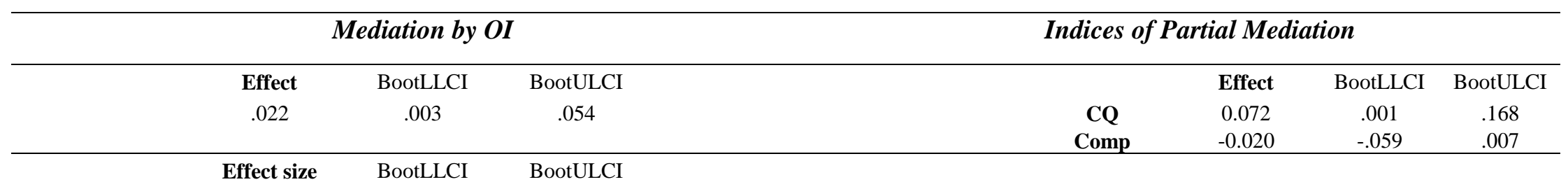

\begin{tabular}{|c|c|c|c|}
\hline & Effect size & BootLLCI & BootULCI \\
\hline $\begin{array}{l}\text { Partially } \\
\text { standardized } \\
\text { indirect effect }\end{array}$ & .025 & .003 & .059 \\
\hline $\begin{array}{l}\text { Completely } \\
\text { standardized } \\
\text { indirect effect }\end{array}$ & .022 & .003 & .052 \\
\hline
\end{tabular}


Table 5.16 Summary results- knowledge sharing

\begin{tabular}{|c|c|c|c|c|c|c|}
\hline & \multicolumn{2}{|c|}{ Mediation model } & \multirow{2}{*}{$\begin{array}{c}\begin{array}{c}\text { Moderation effect of } \\
\text { Comp }\end{array} \\
\text { Kshare }\end{array}$} & \multirow{2}{*}{$\begin{array}{c}\text { Moderation effect of } \\
\text { CQ } \\
\text { Kshare }\end{array}$} & \multicolumn{2}{|c|}{$\begin{array}{l}\text { Moderated mediation } \\
\text { (full model) }\end{array}$} \\
\hline & OI & Kshare & & & $\mathrm{OI}$ & Kshare \\
\hline Constant & -.145 & $.306 * *$ & $.265^{*}$ & $.256^{*}$ & $-.158^{\mathrm{T}}$ & $.331 * *$ \\
\hline Age & .062 & -.011 & .043 & -.043 & .062 & .001 \\
\hline Tenure & $.025 * * *$ & .008 & .006 & $.015^{*}$ & $.025 * * *$ & $.013 *$ \\
\hline Education & $.119 *$ & -.025 & -.044 & -.030 & $.119 *$ & -.024 \\
\hline Task & -.032 & $-.087 * *$ & $-.097 * * *$ & $-.108 * * *$ & -.032 & $-.127 * * *$ \\
\hline $\mathrm{SP}(\mathrm{X})$ & $.069 *$ & $.103 * *$ & & & $.069 *$ & .046 \\
\hline $\mathrm{OI}(\mathrm{M})$ & & .070 & $.115^{\mathrm{T}}$ & -.065 & & -.064 \\
\hline Comp & & & $.338 * * *$ & & & $.347 * * *$ \\
\hline CQ & & & & $.855 * * *$ & & $.878 * * *$ \\
\hline OI x Comp & & & $-.204 *$ & & & $-.198 *$ \\
\hline OI x CQ & & & & .258 & & .197 \\
\hline $\mathrm{R}^{2}$ & .102 & .076 & .173 & .229 & .1018 & .374 \\
\hline $\mathrm{F}$ & $5.805 * * *$ & $3.349 * *$ & $8.650 * * *$ & $12.289 * * *$ & $5.805 * *$ & $13.413 * *$ \\
\hline
\end{tabular}

\begin{tabular}{|c|c|c|c|c|c|c|c|}
\hline & \multicolumn{3}{|c|}{ Mediation by $O I$} & \multicolumn{4}{|c|}{ Indices of Partial Mediation } \\
\hline & Effect & BootLLCI & BootULCI & & Effect & BootLLCI & BootULCI \\
\hline & \multirow[t]{2}{*}{.005} & \multirow[t]{2}{*}{-.003} & \multirow[t]{2}{*}{.019} & CQ & 0.014 & -.019 & .060 \\
\hline & & & & Comp & -0.014 & -.039 & -.001 \\
\hline & Effect Size & LLCI & ULCI & & & & \\
\hline $\begin{array}{l}\text { Partially standardized } \\
\text { direct effect }\end{array}$ & .195 & .018 & .188 & & & & \\
\hline $\begin{array}{l}\text { Completely } \\
\text { standardized direct }\end{array}$ & .174 & .018 & .188 & & & & \\
\hline
\end{tabular}

$\mp \frac{\mathrm{e}}{\mathrm{p}<.10 * \mathrm{p}<.05} * * \mathrm{p}<.01 \quad * * * \mathrm{p}<.001 ; \mathrm{K}=$ knowledge; $\mathrm{SP}=$ Subsidiary power; $\mathrm{OI}=$ Organizational identification; CQ= Cultural intelligence; Comp=Competition; $\mathrm{R}^{2}=$ Coefficient of determination; $\mathrm{F}=\mathrm{F}$ value 
Table 5.17 Summary of hypotheses tests findings

\title{
Seeking Sharing Support
}

\author{
Mediation model
}

H1a: Subsidiary Power has a negative direct effect on knowledge seeking

H1b: Subsidiary Power has a positive direct effect on knowledge sharing

H2a: OI mediates the effect of subsidiary power on knowledge seeking

$\mathrm{H} 2 \mathrm{~b}$ : OI mediates the effect of subsidiary power on knowledge sharing

H3a: Subsidiary power will have a negative indirect effect on interpersonal knowledge seeking mediated through OI.

H3b: Subsidiary power will have a positive indirect effect on interpersonal knowledge sharing mediated through OI.
Non-significant

No

Significant Yes

+ve

Significant

Yes

Non- No

significant

+ve significant

No

Non-

No

significant

Moderated mediation model

H4a: Intra-MNE competition strengthens the negative indirect relationship between subsidiary power and interpersonal knowledge seeking.

H4b: Intra-MNE competition weakens the positive relationship between subsidiary power and interpersonal knowledge sharing.

H5a: Cultural Intelligence weakens the negative indirect relationship between subsidiary power and interpersonal knowledge seeking.

H5b: Cultural Intelligence strengthens the positive indirect relationship between subsidiary power and interpersonal knowledge sharing. 


\subsection{Common Method Variance}

To mitigate the effect of Common Method Variance, procedural measures were used ex ante at the design stage (Chang et al., 2010) as discussed in the Research Design chapter in section 4.5.2. The order of questions were mixed and complicated regression models were used which made it unlikely for respondents to visualize interactions and non-linear effects. Also, respondents were assured of the anonymity and confidentiality of the study, and any form of ambiguity was removed. Additionally, two statistical techniques were also used ex post to assess CMV: Harman's one factor test and Lindell \& Whitney's (2001) marker variable technique. Harman's one-factor test was performed with an exploratory factor analysis for all the constructs to check if one single factor accounts for the majority of the variance. If one single factor accounts for more than $50 \%$ of the variance of the factors in the factor analysis, it indicates that CMV is present in the study (Harman, 1967). The results for this test are in Table $1 \& 2$ (Appendix 8). It is observed that for the knowledge seeking variables, the percentage of variance accounted for by the largest single factor is $10.17 \%$ and for the knowledge sharing model variables, it is $11.46 \%$, which are well below the $50 \%$ threshold. Eigen value measures the amount of variation in the total sample accounted for by each factor. All nine factors in each of the knowledge seeking and sharing have eigenvalues greater than 1. These results indicate that the study does not suffer from the CMV issue.

Following Lindell and Whitney's (2001), I have checked the correlations and partial correlations for the variables measured with perceptual measures. This is in line with Malhotra et al. (2006), Najafi-Tavani, Giroud, and Sinkovics (2012), and Noorderhaven and Harzing (2009). First, I inspected each variable's zero-order correlation for statistical significance. Later, the partial correlation between the variables controlling for the marker, "Fashion Consciousness" was checked. It was found that all the significant zero-order correlations for the variables remained significant for the partial correlations. Thus, it can be concluded that common method variance does not play a role in the findings (Lindell \& Whitney, 2001; Noorderhaven \& Harzing, 2009).

Tables 3 and 4 (Appendix 8) present zero-order and partial-correlations respectively for the knowledge seeking model. Similarly, Tables 5 and Table 6 (Appendix 8) present the zero-order and partial-correlations respectively for the knowledge sharing model. 


\subsection{Summary}

After screening and cleaning of the data I undertook CFA to present validity and reliability of the measures used in my study. All the latent variables were imputed to use the PROCESS Macro to test the proposed hypotheses. I found mixed evidence for my proposed conceptual model. In the next chapter I will discuss the key findings and their theoretical and managerial implications. 


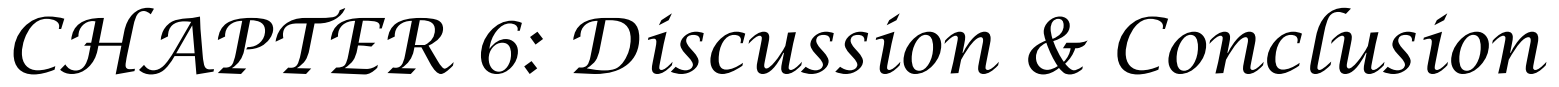

\subsection{Introduction}

This chapter begins with a summary of the findings from data analysis. This is followed by presenting theoretical and managerial implications. Then limitations of the study and future research avenues are presented before concluding the thesis.

\subsection{Key Findings}

Using a Knowledge Based View and Social Identity Theory, I argued that employees' cross border knowledge transfer (seeking and sharing) behaviour across subsidiaries within an MNE is influenced by the subsidiary characteristic which is subsidiary power. I proposed that employees' identification with the organization, the subsidiary, enables this effect. I argue that subsidiary power is a strong driver of employees' identification with the subsidiary which then influences their knowledge exchange behaviour across units. I then introduce two boundary conditions; cultural intelligence and intra-MNE competition (between the subsidiaries) which, I propose, would influence the effect of subsidiary power on knowledge exchange.

I then tested the dependent variable knowledge transfer/exchange with two different models by separating it into two different variables; knowledge seeking and knowledge sharing, as I believe that these are two different behaviours and need to be examined separately. The findings from the hypotheses tests suggest that subsidiary identification mediates the effect of subsidiary power on the knowledge seeking behaviour of employees, whereas, it doesn't mediate the effect on knowledge sharing.

The direction of the effect of subsidiary power on knowledge seeking has been found to be contrary to what was hypothesized. It was argued that relative subsidiary power is negatively 
associated with knowledge seeking. But it was found that the effect is positive. While the effect on knowledge sharing is found to be positive as expected. Testing the conditional effect of cultural intelligence of the employees and intra-MNE competition on the indirect effects of subsidiary power on knowledge seeking and sharing, I found that cultural intelligence does moderate the effect on knowledge seeking and competition moderates the effect on knowledge sharing. However, as hypothesized, competition doesn't influence the effect on knowledge seeking and cultural intelligence doesn't affect the effect on knowledge sharing.

Below I discuss each of the findings with respect to the research questions.

\subsubsection{Direct effects of subsidiary power}

The first hypothesis examined the effect of subsidiary power on knowledge seeking and sharing across subsidiaries. Based on the literature it was assumed that subsidiary power will have a negative effect on individual knowledge seeking and a positive effect on knowledge sharing.

I argued that due to the dynamics of power within the MNE, individual actors in the subsidiaries use political behaviour to enhance the power of their subsidiary during interactions across the MNE (Conroy, Collings \& Clancy, 2018; Dörrenbächer \& Geppert, 2011). Individual knowledge transfers are such socio-political interactions (Dörrenbächer \& Geppert, 2011). Subsidiary members are more likely to seek knowledge from a subsidiary which is relatively high in power and possesses valuable knowledge. Therefore, it was assumed that high-power subsidiary members are less likely to engage in knowledge seeking and engage more in knowledge sharing. That is, there is a negative relationship of subsidiary power with knowledge seeking and a positive relationship with knowledge sharing.

The findings suggest no evidence of a direct relationship between subsidiary power and individual knowledge seeking. However, as predicted, the direct relationship with knowledge sharing is found to be positive and significant. Generally, knowledge held by a high-power subsidiary is perceived as more valuable and superior (Frenkel, 2008; Mudambi \& Navarra, 2004), which leads to members of another subsidiary which is less powerful, seeking knowledge from it (Borgatti \& Cross, 2003). More knowledge seeking by members of low-power subsidiaries leads to more sharing of knowledge. Also, high-power members share more knowledge with the lowpower members due to their perception that the low-power subsidiary knowledge is inferior to theirs. Further, knowledge outflows from the low-power subsidiary is less due to low-power subsidiary members sharing less or hiding knowledge and information from the high-power 
members due to fear of coercive action by the high-power. This causes more knowledge sharing by the high-power subsidiary members. Thus, the findings substantiate my arguments that subsidiary power has a positive direct relationship with knowledge sharing.

\subsubsection{Indirect effect of subsidiary power}

The findings support my prediction that organizational identification of employees mediate the effect of subsidiary power on knowledge seeking. Whereas, support for the effect of subsidiary power on knowledge sharing through identification was not found.

As expected, subsidiary power influences individual employee's knowledge seeking from another subsidiary through its effect on employee's organizational identification. Employees make sense of their power as well as that of other subsidiaries based on certain organizational cues such as network position of the subsidiary, control over resources, influence over HQ's decisions (Fiol et al., 2001). This generates individual perception or mental models about the power of their own subsidiary as well as other subsidiaries within the MNE (Fiol et al., 2001).

As employees perceive higher subsidiary power, their identification with the subsidiary increases. As discussed, high power contributes to the distinctiveness and prestige of the subsidiary, which makes its construed external image more attractive (Bartels et al., 2007; Dutton et al., 1994; Smidts \& Pruyn, 2001). According to Social Identity Theory (Turner, 1975), this distinctiveness accentuates the existences of their own subsidiary and strengthens the differences and similarities with the other subsidiaries.

This leads employees to engage in self-categorization, distinguishing between the in-group (own subsidiary) and the out-groups (other subsidiaries), and increasing the in-group (subsidiary) identification. Higher in-group or subsidiary identification, as in this case, promotes individual behaviour that highlights inter-group differences and minimizes in-group differences. They tend to display behaviour that is favourable and beneficial to the subsidiary (Ashforth et al., 2008; Bartels et al., 2007). Knowledge is a source of a subsidiary's competitive advantage and also a source of power (Argote \& Ingram, 2000; Mudambi \& Navarra, 2004). Employees with high identification are likely to protect the resources and capabilities that confer power to the subsidiary or would refrain from displaying any kind of behaviour that questions their power status. Therefore, the findings suggest that subsidiary power will influence employees' knowledge seeking through its effect on employees' organizational identification. 
Contrary to my hypothesis, the indirect effect of subsidiary power on knowledge seeking through identification is found to be positive rather than negative. My prediction was based on the social identity theory, which involves the motive of self-enhancement (Terry \& Hogg, 1996) or opportunity focused personal identification (Ashforth et al., 2016). I had argued that due to identification and these motives involved, individuals are likely to associate themselves with the more powerful ones. By building relationships with the more powerful, they try to enhance their power. Also, it was assumed that in order to protect their status of power, higher power members would refrain from seeking knowledge due to fear of displaying their knowledge gap or incompetency.

However, the positive relationship may be due to global product mandates of Indian R\&D subsidiaries. As foreign multinationals are setting up high-end R\&D projects in India, Indian R\&D units are beginning to cater to global product needs and therefore they form an integral part of the global knowledge networks. Thus, the need to acquire more knowledge and information might be the reason for increased knowledge seeking when the power of the subsidiary is high due to holding global mandate. Another reason for the positive relationship may be the cultural context of India. Traditionally, India is a high power distance country where people have respect and acceptance of hierarchy and power differences (Chhokar, 2007). Although a multinational organizational culture advocates and inculcates less power differences among employees, local employees also derive their values from the national culture in which the organizational culture is nested (Pothukuchi, Damanpour, Choi, Chen, \& Park, 2002).

Power distance refers to the degree to which individuals and groups accept and expect that power is distributed unequally (Hofstede, 2001). The cultural value of power distance is fundamental to relationships and integral to organizations and organizational processes (Keltner, Gruenfield, \& Anderson, 2003; Daniels \& Greguras, 2014). In high power distance cultures, individuals with power are perceived as superior and inaccessible (Hofstede, 1980; Daniels \& Greguras, 2014). Subordinates hesitate to confront their supervisors with questions or inputs (Hofstede, 1991). Studies have found a negative relationship between high power distance and feedback seeking behaviour (Hwang \& Francesco, 2010; Morrison, Chen \& Salgado, 2004). In a high power distance society, low power members are found to rely less on vertical sources for guidance (Smith et al., 2005; Hwang \& Francesco, 2010). This has been observed among students from high power distance cultures who prefer to seek feedback from fellow students rather than 
from their professor (Hwang \& Francesco, 2010). Therefore, when the employees perceive their subsidiary power to be relatively low, they shy away from interaction with a relatively high power subsidiary member. This is likely to lead to less knowledge seeking behaviour.

Further, power distance determines the leadership style. Those with power in a high power distance culture expect an autocratic or paternalistic leadership approach (Hofstede, 1980). Previous research suggests paternalism to be a prevalent management practice in India (Mathur, Aycan, \& Kanungo, 1996). Those with high power adopt a paternalistic management style where the highpower leader guides and protects the wellbeing of the subordinates in the manner resembling a parent. The powerful authority nurtures, cares and displays mixed qualities of benevolence and authoritativeness. Whereas, the subordinates are expected to be loyal and conforming to the authority figure (Pellegrini, Scandura, \& Jayaraman, 2010).

Therefore, members of a high-power subsidiary in India are likely to display such a paternalistic attitude towards low-power subsidiary members to underscore their authoritative power over them. This would promote behaviour which is more communication focused to increase connectedness with the low-power members. As Pellegrini, Scandura and Jayaraman (2010) posit, the outcomes of paternalistic relations are not necessarily economic transactions. The objective behind the relationship goes beyond economic exchange. So individuals use different communication channels to gain face and show their knowledge and ability (Hwang, Francesco, \& Kessler, 2003). Therefore, increasing communication and connectivity through reaching out to the low-power subsidiary members for knowledge gives them the opportunity to underline their power and authority over the low power members and further nurture their power status to maintain continued power in the MNE.

Additionally, this positive relationship between subsidiary power and knowledge seeking may also be due to the Indian culture being more performance oriented (Chhokar, 2007; House, Dorfman, Javidan, Hanges, \& de Luque, 2013). In such societies, innovation, high standards and performance improvement are encouraged and rewarded (Javidan, 2004). So, when they identify with a high-power subsidiary, their orientation to high performance and competitiveness encourages them to strive for improving their performance and keep up to their high power status. This encourages more knowledge seeking to meet their demands for knowledge and information to accomplish their work. 
In contrast to my hypothesis, there was no evidence of mediation of the effect of subsidiary power on knowledge sharing. This implies that when it comes to the sharing of knowledge with another subsidiary member, the power of a subsidiary doesn't influence knowledge sharing due to employees' identification with the subsidiary. Although subsidiary power has a positive and significant relationship with identification, there was no significant relationship detected between identification and knowledge sharing. This indicates that the knowledge sharing behaviour of employees is not related to their identification with the subsidiary or its power. This finding may also be due to cultural factors in India.

Knowledge sharing behaviour is much influenced by culture (Hofstede, 2001; Hutching \& Michailova, 2004). The dimension of humane orientation is crucial for sharing. Human orientation is "the degree to which individuals in organizations or societies encourage and reward individuals for being fair, altruistic, friendly, generous, caring, and kind to others" (House, Javidan, Hanges, \& Dorfman, 2002, p. 6). Indian culture is deeply rooted with human orientation and therefore scores very high on this dimension (Chhokar, 2007). Human oriented people are supportive, more generous, compassionate and concerned about the well-being of others (House, Hanges, Javidan, Dorfman, \& Gupta, 2004). This dimension emphasizes empathy for others by providing time, money or other resources such as assistance when needed (Dorfman, Javidan, Hanges, Dastmalchian, \& House, 2012).

Furthermore, Indians also score high on the dimension of institutional collectivism (Chhokar et al., 2007). Institutional collectivism is the degree to which organizational and societal institutional practices encourage and reward collective distribution of resources (House et al., 2002, p. 6). This dimension emphasizes the belief that the self should be interdependent with others and should have duties and obligations towards the collective, over and above personal interests (Waldman et al., 2006). It is different from in-group collectivism which is the degree to which individuals take pride of the family or the organization to which he/she belongs.

Institutional collectivism promotes group harmony and co-operation among organizational members as they value interdependent relationships and achievement of the greater goal of the collective institution. Therefore, Indian employees are less likely to hide knowledge from other subsidiary members and more likely to share knowledge with them whenever there is any request for knowledge. Due to these culture-specific influences on knowledge sharing behaviour, 
knowledge sharing may not be influenced by subsidiary power through identification of the employees.

Based on the causal steps strategy of analysing mediation, the degree of mediation of the knowledge seeking model is termed as 'complete mediation'. But, Hayes (2018a) asserts that this term is flawed and therefore we should avoid using it. By the term 'complete mediation', we understand that the effect of subsidiary power on knowledge seeking is completely mediated by organizational identification. That is, identification is the only mechanism through which power affects seeking and it is confirmed that there is no other mechanism that would do. However, in reality, this is not the case. A complete mediation model does not provide any information about the existence or absence of other possible subsidiary power mediators (Rucker, Preacher, Tormala, \& Petty, 2011). There can be other investigators who make the same claim with other mediators. Thus they don't hold any theoretical meaning. Therefore, I have decided not to use these concepts to interpret my mediation results.

\subsubsection{Moderation effect of cultural intelligence}

An important finding was that CQ moderates the indirect effect of subsidiary power on knowledge seeking by moderating the direct effect of organizational identification on knowledge seeking. The effect is positive and significant and at higher levels of CQ. This implies, for employees having different CQs, specifically those having CQs at higher levels, the effect of subsidiary power on knowledge seeking will vary and the effect is positive. CQ will strengthen the effect of subsidiary power on knowledge seeking. The higher the CQ of employees, the stronger will be the positive effect of organizational identification on knowledge seeking and also the indirect effect of subsidiary power. Two employees with the same level of identification and subsidiary power will have different levels of knowledge seeking if their CQ levels are different (at higher levels).

CQ enables an employee to work effectively in a culturally diverse climate. Individuals with higher CQ genuinely enjoy interacting with culturally different people. They are more motivated, open-minded, curious, and enjoy learning new and different things. Organizational identification leads people to engage in self-categorization based on typical group characteristics which promotes biased behaviour. As CQ enables cultural perspective taking, it reduces any kind of bias or stereotyping which arises due to OI or categorizing between in-group and out-group members. This improves exchange relationships (Bogilović, Černe \& Škerlavaj, 2017; Imai \& Gelfand, 2010; Mor, Morris, \& Joh, 2013). Better understanding of another culture helps them to overcome any kind of 
prejudices to collaborate with culturally different others (Ang \& Dyne, 2008; Chen \& Lin, 2013). Also, a culturally intelligent employee possesses the necessary knowledge about the other culture which builds his/her self-confidence to deal with culturally diverse people and situations. Effective communication is the cornerstone of any cross-cultural interaction (Chua et al., 2012). They are therefore more confident that they can act more appropriately and deal with any kind of ambiguity and uncertainty that is mostly common in any cross-cultural interaction (Bücker et al., 2014; Groves et al., 2015).

It is also observed that the direct effect of cultural intelligence on knowledge seeking while controlling for other factors is positive and highly significant. Due to CQ, employees can easily overcome any hesitation to interact with a subsidiary member from a high-power subsidiary due to experiencing high power distance. This not only increases and makes cross-cultural communication easier, but also more effective. Therefore, a member of a low-power subsidiary can conquer any inhibition that arises due to their identification with their subsidiary and experience of high power distance and thereby engage more in knowledge seeking. Similarly, knowledge seeking by a highpower member will also increase as communication improves due to better understanding and less

ambiguity in the exchange relationship. Thus, the effect of subsidiary power on knowledge seeking through identification is stronger in the presence of high CQ. Additionally, considering the presence of intra-MNE competition, it is observed that moderation by CQ takes place at higher levels of CQ when intra-MNE competition is held constant at moderate and lower levels.

However, although there was no evidence of mediation of the effect of subsidiary power on sharing, I tested for moderation by CQ to examine if there is an effect for employees with varying levels of CQ. No effect was found. As observed, employees share knowledge due to their humane orientation and institutional collectivism. Although these cultural characteristics are not particular aspects of CQ, they are likely to be highly correlated with CQ. Culturally intelligent people are more empathetic to people from other cultures. They are good at perspective taking and due to their understanding of other cultures they are more cooperative. Therefore, even for employees having different levels of CQ, their knowledge sharing is not influenced by subsidiary power through identification.

\subsubsection{Moderation effect of intra-MNE competition}

There was no evidence of mediation of the effect of subsidiary power on knowledge sharing by organizational identification. However, an examination of moderation effect by intra-MNE 
competition has found that moderation exists. It is revealed that the effect of subsidiary power on sharing through identification exists depending on intra-MNE competition, which was not included in the simple mediation model (Hayes, 2018a). That means, whether or not different levels of subsidiary power will have different effects on knowledge sharing through identification, depends on their perceived intra-MNE competition. This relationship works only in the presence of competition.

The moderation effect is found to be negative and at higher levels of intra-MNE competition. This implies, intra-MNE competition weakens the effect of identification and power on sharing. Earlier, it was found that when it comes to knowledge sharing, subsidiary power through identification does not have an effect. Employees share knowledge regardless of any identification with their subsidiary due to their humane orientation and institutional collectivism. Only in the presence of high intra-MNE competition, is knowledge sharing influenced by the power of the subsidiary and their identification with the subsidiary. That is, for employees perceiving different levels of intra-MNE competition (at higher levels), the effect of subsidiary power on sharing will exist and such effect will be weak.

This is in line with my prediction. Intra-MNE competition creates barriers to knowledge sharing. Knowledge is a source of power. By sharing knowledge with a competitor subsidiary member, the subsidiary risks the loss of its unique knowledge that may give it a competitive advantage in intra-MNE bargaining for power. Also, the expectation of reciprocity from the competitor is less. Thus, there is a fear of benefiting the competitor and losing one's ability to compete. A competitor subsidiary is unreliable and there is lesser trust of how they would use the knowledge shared (Lai et al., 2014; Chen \& Hung, 2010). Due to intra-MNE competition, subsidiaries may act opportunistically. Lack of trust in the competitor subsidiary deters sharing. Therefore, in the decision to share knowledge with a competitor, the expected cost outweighs the benefits which discourages knowledge sharing. If we take into consideration the presence of CQ, it is observed that intra-MNE competition moderates the effect of subsidiary power on knowledge sharing at higher levels of competition when CQ is held constant at moderate and lower levels.

There was no evidence of moderation of the effect of OI on knowledge seeking by intraMNE competition. This means, I did not find evidence for the positive effect of OI on knowledge seeking or any indirect effect of SP on knowledge seeking with respect to different levels of intraMNE competition. That means, two employees who perceive similar subsidiary power and 
identification, but different levels of perceived intra-MNE competition may have similar levels of knowledge seeking. Also, the direct effect of intra-MNE competition on knowledge seeking is found to be insignificant. Thus, regardless of the level of intra-MNE competition, it does not impact any knowledge seeking decision.

\subsection{Advances and Contribution to Research}

\subsubsection{Cross-border individual level knowledge transfer in the MNE}

By examining cross border individual knowledge transfer in the MNE, this study advances our understanding about individual knowledge transfer behaviour as well as knowledge transfers at the organizational level, as individual transfers are the micro-foundations of organizational level knowledge transfers. At the beginning, I carried out a comprehensive literature review on knowledge transfers in MNEs encompassing studies at all levels of analysis and all antecedents. Doing so, I systematically organized and divided the literature by arranging it into a two-by-two matrix based on antecedents and the outcomes (knowledge transfer) at two levels, individual and organizational. This is a first of its kind attempt to explore, elucidate and present research carried out in knowledge transfer through the two level matrix.

A thorough analysis of the research carried out so far revealed that studies have primarily focused on a single level of analysis, organizational antecedents on organizational knowledge transfers and individual antecedents on individual transfers; while multilevel studies are emerging. In particular, it was found (and also being highlighted by similar reviews) (Michailova \& Mustaffa, 2012) that individual level knowledge transfers in MNEs have received relatively very little attention. This indicates a lack of complete understanding of the knowledge transfer process in MNEs as individuals are the micro actors and individual transfers are the cornerstone of transfers at the organizational level (Argote \& Ingram, 2000). The literature review helped validate the need to focus my attention on individual knowledge transfers in MNEs.

MNEs are complex organizations with a diversity of contexts and both local and global forces exercising their influences. Organizational separation in the MNE poses a major hindrance to knowledge transfers both individual and organizational (Ambos \& Ambos, 2009: Dasí et al., 2017; Gupta \& Govindarajan, 2000). Therefore, I focused on investigating individual knowledge transfers across borders and thoroughly reviewed literature on the subject. This probed into the factors that emerge due to the separation of MNEs involving diverse contexts of operation which may influence individual transfer decisions. 
My review revealed that a few studies which have touched on this subject have basically dealt with antecedents at the individual level which are characteristics such as personality, expertise, attitudes, motivation and relationships. One distinct group has evolved around organizational antecedents like HRM practices that can govern individual knowledge transfers in MNEs. Some of the studies have focused on context specific organizational factors like culture and language, which are idiosyncratic to MNEs.

In my work, I have identified certain organizational level factors such as subsidiary power and intra-MNE competition, and brought in a relevant individual level factor, cultural intelligence, which emanates due to the complex web of interdependent organizational units and employees located in different contexts, and operating with diverse responsibilities, objectives and interests, which is typical in an MNE. Through this, I contribute to advancing our knowledge and understanding of individual level knowledge transfers in MNEs.

Individual knowledge transfers were examined with two activities, knowledge seeking and knowledge sharing. I believe that by treating knowledge transfers as primarily sharing, most of the literature provided a fragmented view of the process. As they are two distinct activities, I examined them separately. The findings also substantiate this standing as the impact of factors on knowledge seeking are found to be different from those of knowledge sharing.

Subsidiary power is directly and positively associated with sharing knowledge but no direct relationship was found with seeking knowledge. That means, when it comes to decisions about seeking, there are other mechanisms through which subsidiary power exerts its influence. But knowledge sharing decisions are directly influenced by subsidiary power. The mechanism through which subsidiary power is found to impact knowledge seeking is organizational identification. Subsidiary power has a positive effect on organizational identification which then has a positive effect on knowledge seeking. This implies, when it comes to the decision to seek knowledge from another subsidiary member, the power of the employees' own subsidiary will have an impact on the decision based on the employees' identification with the subsidiary. Whereas, for knowledge sharing, whether or not the employee identifies with the subsidiary is less relevant.

I brought in two boundary conditions; intra-MNE competition and cultural intelligence of the employees, which were found to influence the effect of subsidiary power on knowledge seeking and sharing. Seeking decisions based on subsidiary power and identification were found to vary for employees having different levels of cultural intelligence but not for different levels of 
perceived intra-MNE competition. An interesting finding for knowledge sharing was; although it was found that any impact of subsidiary power on knowledge sharing is not based on mediation by identification of employees, but in the presence of intra-MNE competition, the indirect effect of subsidiary power on sharing through identification works. That is, for employees who perceive different levels of intra-MNE competition, their levels of knowledge sharing due to subsidiary power will be based on their identification with the subsidiary, and the levels will be different. Due to perceptions of competition, subsidiary power will impact knowledge sharing decisions through employees' identification with the subsidiary.

\subsubsection{Contribution to social identity theory}

For examining individual knowledge transfer in MNEs, I draw on the Social Identity Theory (SIT). Organizational identification, an important component of SIT was used to explain the impact of certain organizational level factors on knowledge transfer behaviours of employees across subsidiaries. Organizational identification is the degree to which employees define themselves by the same attributes they believe define the organization (Dutton et al., 1994, p. 1). Certain distinctive characteristics of the organization which are salient to it enhances employees' identification with the organization. Employee's organizational identification in the MNE has been the subject of many discourses. It is found that employees tend to identify with both the MNE and the local subsidiary, although to different degrees.

Due to organizational separation of MNEs, organizational identification of subsidiary employees in the MNE may play a crucial role in knowledge transfers within the MNE. Earlier studies which have used organizational identification to explain knowledge transfer in MNEs have applied a relational perspective (Argote \& Kane, 2009; Reiche et al., 2015). Subsidiary member's identification with the higher-order unit, the MNE, which may be called the superordinate social identity, promotes coordination between members belonging to different subsidiaries and thus facilitates knowledge transfers between them. Reiche et al. (2015) used this shared identity of individual employees in subsidiaries to examine the effect of shared language on knowledge transfers between members of different subsidiaries. This SIT approach is more relational than content-based, where identity is based on distinctive organizational characteristics (Argote \& Kane, 2009)

In this study I use a content-based SIT approach to examine knowledge transfers in MNEs. Organizational identification is based on power and such other resources of the subsidiary which 
are distinct to it. The different geographic, socio-cultural, and economic environments in which subsidiaries of an MNE are located, contribute to their distinctiveness and lead to subsidiary members' identification with the subsidiaries. As found by this study, identification impacts individual knowledge transfers in the MNE. This is a novel attempt at using SIT for examining knowledge transfers in MNEs. By using this approach of identification which emerges due to separation of the subsidiaries in an MNE, I contribute and enhance our understanding of a different facet of how identification in organizations, more specifically in MNEs, can impact knowledge transfer.

\subsubsection{Contribution to knowledge governance approach}

KGA draws upon Coleman's bath tub model of knowledge transfer (Coleman, 1990; Foss, 2007; Minbaeva et al., 2012) presents the knowledge transfer process at two levels: organizational and individual levels. Primarily, it argues that macro level factors create micro level conditions which result in micro level knowledge exchange through changes in the individual behaviour leading to macro level knowledge flows. Most of the literature on knowledge transfer is at single level (Quad 1 and Quad 3: Table 2.1) while multilevel studies (Quad 2 and Quad 4: Table 2.1) are emerging. I have specifically focused on Quad 4 to propose a new set of organizational level factors that influence interpersonal knowledge exchanges within the MNE which in turn influence organisational level knowledge transfer in line with the micro foundations approach (Felin \& Foss, 2005).

Along with formal and informal mechanisms of knowledge governance, the new set of quasiformal organizational antecedents are shown in Figure 6.1. Formal mechanisms such as HRM practices, knowledge management tools and direct awards of more subsidiary autonomy are under the direct control of the MNE and thus influenced mainly by internal resources and requirements. Being direct control mechanisms, less perception management is required as rewards and other outcomes are directly associated with such mechanisms, and thus can be considered as transactionbased mechanisms (Husted et al., 2012). On the other hand, informal mechanisms such as organisational culture and values are intended to enhance commitment and thus need more for perception management. Organizations use these as indirect control mechanisms. In addition to internal requirements and resources, some external factors such as an individual's personality traits are likely to influence their impact. 
Figure 6.1 Quasi-formal identity based mechanisms

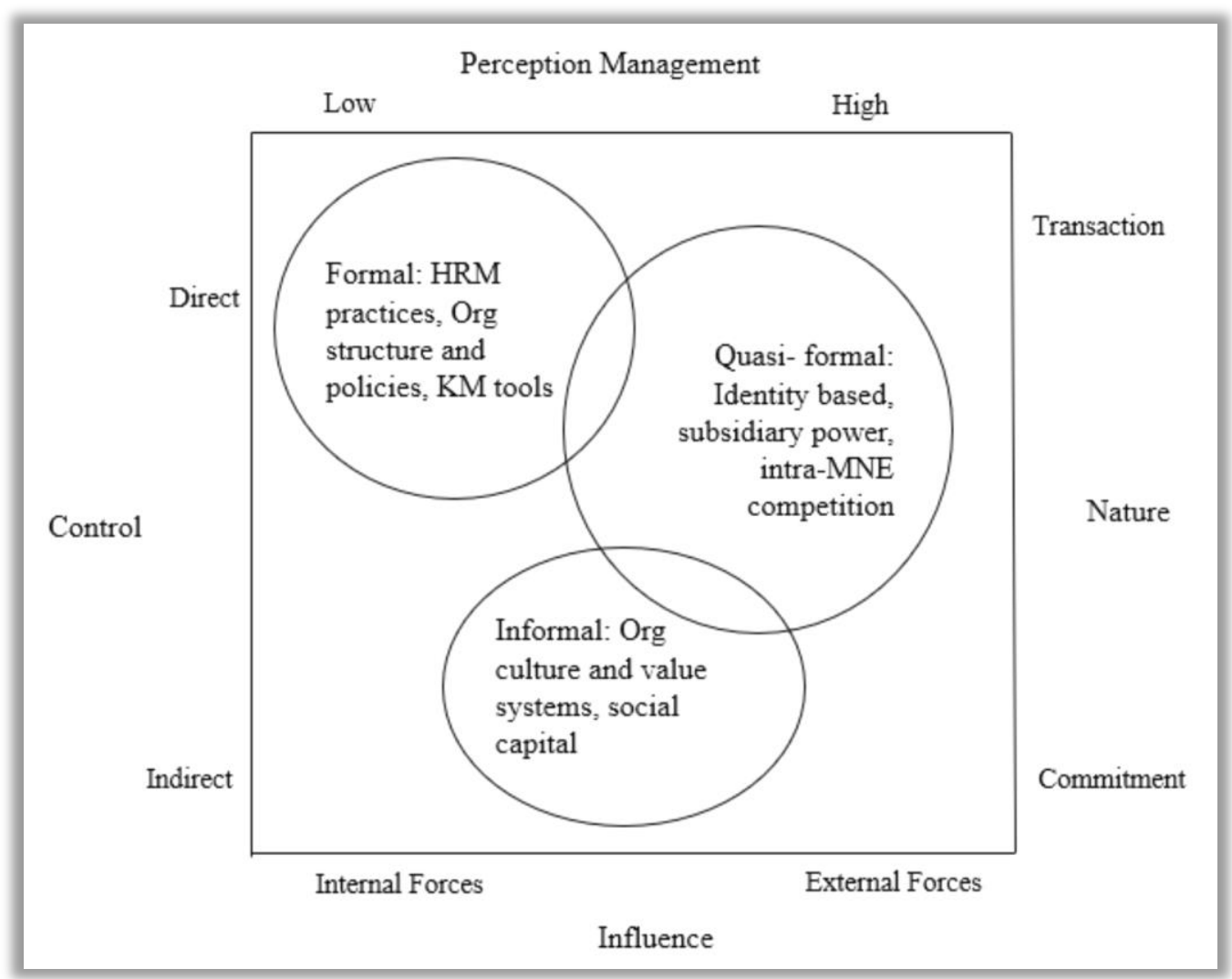

Source: Author's compilation from the review of literature

Quasi-formal mechanisms are based on the premises of Social Identity Theory. These antecedents, subsidiary power or intra-MNE competition, like other formal antecedents, are not entirely determined or influenced by the focal subsidiary but depend on other external actors as well. I call these factors identity-based quasi-formal knowledge governance mechanisms.

Subsidiary power can also emerge as a formal organizational mechanism. Sometimes it is possible that the MNE may conspicuously grant more power, autonomy or decision-making rights to a particular subsidiary by providing mandates or appointing it as a centre of excellence and endowing it with formal responsibilities of knowledge creation and organizational level knowledge transfers (Ciabuschi et al., 2010). However, subsidiary power cannot be labelled as fully formal.

Similar to an informal factor but unlike a formal factor, it is semi-permanent in nature and much harder to change for a particular subsidiary (Foss et al., 2010). Generally, to influence knowledge sharing behaviour, managers can use changes in formal factors or mechanisms as 
signals to organizational members. But it is not directly possible for a subsidiary to manage or change its power to influence individual knowledge exchange behaviour. Therefore, I call subsidiary power quasi-formal.

I see these quasi-formal 'identity-based mechanisms' as in-between the transaction-based and commitment-based knowledge governance mechanisms (Husted et al., 2012). Transactionbased mechanisms use tangible and explicit incentives to influence knowledge sharing behaviour, while commitment-based mechanisms use encouragement and cognitive stimulation. Whereas, 'identity-based mechanisms can use employees' organizational identification or perception of these identity-based factors to influence knowledge sharing behaviour, such mechanisms require higher levels of perception management.

Knowledge governance mechanisms have a psychological or cognitive influence on the individual which motivates them to engage in knowledge sharing. Individual's perceptions, attitudes, desires, and such other cognitive effects impact knowledge sharing behaviour. I add to the KGA and identify and bring in a new set of organizational level antecedents that can be used to govern individual level knowledge exchanges in the organization. Additionally, I have used the Social Identity Theory to explain the underlying individual level micro-behavioural process. Organizational identification explains the micro-level psychological processes of selfcategorization and self-enhancement that explain the impact of certain organizational level factors on individual knowledge exchange behaviour.

\subsubsection{Contribution to understanding the context of study}

The study was conducted in India on Indian employees. It helped us gain insights to understanding knowledge transfer behaviours of Indian employees. From the findings it is observed that the organizational behaviour of Indian employees is influenced by the national culture to a great extent.

Collectivist culture is found to be an important antecedent of organizational identification (Baker, Carson \& Carson, 2009). Due to high in-group collectivism of Indian culture (Chhokar, 2007), loyalty and identification with the organization is high. As found, they are more likely to draw on salient distinctive characteristics of their subsidiary, such as power. When it comes to seeking knowledge from another subsidiary, which is equivalent to seeking help, the power of their subsidiary impacts their decision through their identification to the subsidiary.

Not only with in-group collectivism, Indians score high in institutional collectivism as well (Chhokar, 2007). While in-group collectivism facilitates identification with the organization and 
impacts seeking behaviour, the influence of institutional collectivism is observed in their knowledge sharing behaviour. They value that, for achieving the common goals and objectives of the MNE, which the subsidiaries share, the work processes need to be interdependent. They adopt a more cooperative attitude and seek harmonious relationships with other subsidiaries which encourages Indian employees to share knowledge.

\subsection{Managerial Implications}

As put by Foss et al. (2010), to gain maximum benefits from knowledge sharing, it is crucial that managers and decision makers are aware of different situations, as well as the appropriate governance mechanisms that may be deployed to achieve the desired knowledge sharing behaviour.

By adding a new set of antecedents to the knowledge governance approach, this research directly contributes to management of individual knowledge transfers in the MNE. The concepts of subsidiary power, intra-MNE competition and CQ are not new to managers. However, by linking them to cross-border individual knowledge transfers for the first time, this study draws attention and adds these identity- based factors to the management of individual knowledge transfers in MNEs.

My findings suggest that managers should consider the power of a subsidiary and intra-MNE competition while governing individual knowledge transfers across subsidiaries. Whether directly or indirectly, subsidiary power influences knowledge seeking and sharing decisions of individuals. In a formal parlance, subsidiary power is a feature of the subsidiary, although temporary. The HQ can formally endow the subsidiary with power through mandates, resource allocations or rights in MNE decision making. At the same time, the HQ can divest a subsidiary of its power. Thus, HQ managers can influence individual knowledge transfers across subsidiaries or to and from the HQ.

However, subsidiary managers cannot change the power of their or another subsidiary. Managers can use perception-based management techniques to influence employees' perception of power or intra-MNE competition. Managers can hold focused discussions around the role of their own subsidiary or another subsidiary, and implement effective socialization mechanisms (Birkinshaw et al., 2000) which can help change or create perceptions. Also, to some extent, it is possible to influence identification of employees with the subsidiary. Managers can enhance organizational identification of employees by communicating to them about organizational successes, goals and values. Again, rotation of individuals, such as international assignments across subsidiaries, can help enhancing their identity with the superordinate authority, the MNE (Richter, 
West, Dick, \& Dawson, 2006). Managers can manipulate perceptions of subsidiary power, intraMNE competition or use organizational identification in order to encourage or discourage individual level knowledge transfers within the MNE.

I found that in a high power distance culture, high-power subsidiary employees are more likely to seek knowledge than low-power subsidiary employees, due to identification with the subsidiary. This is due to high-power distance culture where employees who identify with a relatively low-power subsidiary shy away from seeking knowledge from a high-power subsidiary. Sharing of knowledge is found to be high among high-power subsidiary employees. This is due to other relatively low-power subsidiary members seeking knowledge from the high-power subsidiary. Unlike knowledge seeking, a decision to share does not depend on the identification of employees with the subsidiary. In a culture which is high in institutional collectivism, identification matters less for impacts of subsidiary power.

If a subsidiary aims to encourage knowledge inflows or seeking by its employees when the power of the subsidiary is low, it can take measures to dilute the identification of the employees with the subsidiary and strengthen identification with the MNE. This can be done through socialization tactics that increase social interaction across subsidiaries, cross-border employee mobility, or practices where employees from different parts of the MNE are put together and given responsibilities which require them to work towards a common greater goal (Smale et al., 2015). This will eliminate any hesitation to interact with the high power subsidiary member. Or, if a high power subsidiary wants its employees to decrease its inflow of knowledge, it can manipulate and project the other subsidiary as more powerful which will deter knowledge seeking of its employees who are in a high power distance culture. Also, to strengthen employees' seeking behaviour due to power, an organization can implement training programmes that can help its employees develop their cultural intelligence.

A high-power subsidiary in a highly institutional collectivist country should be aware that its knowledge outflows are greater due to its employees engaging in sharing, because of high power. If it wants to control knowledge outflows, it needs to design management tools to curb the influence of institutional collectivism. It should be noted that in case of knowledge sharing, increasing or reducing identification with the subsidiary will not have any influence on employees' sharing behaviour. However, if the employees perceive intra-MNE competition, their sharing behaviour due to power will be weakened and it will work through their identification with the subsidiary. 
Therefore, to reduce knowledge outflows due to power, mangers can project other subsidiary/ies as competitors and increase employees' identification with their own subsidiary. Whereas, to further increase knowledge outflows, the managers can project the subsidiaries as non-competitors and strengthen employees' identification with the MNE rather than with their own subsidiary.

Thus, subsidiary managers can influence individual knowledge transfers either through mechanisms which manage organizational identification of the employees or through manipulating employees' perceptions of their subsidiary vis a' vis another subsidiary or HQ depending on their knowledge transfer objectives.

\subsection{Limitations}

Like any other research, my study has some limitations which need to be considered while interpreting the results.

Considering the multilevel and nested nature of the variables used in the model, although lesser Intra-class Correlation of the data is found (close to zero), a multilevel research method would have been the ideal approach for this study. To have an adequate power of Hierarchical Linear Modeling (HLM) (i.e. 0.90) to detect cross-level (two level) interactions, a sample of 30 groups with 30 individuals each is necessary (Bassiri, 1988). But, if the number of groups increases, the number of observations required per group will be reduced. For example, to attain a power of 0.90, 150 groups will require 5 respondents per group. In this study, data was collected from 40 groups and respondents for each group varies to a great extent. Therefore, it was not viable to use the HLM data analysis technique. Also, although the variables subsidiary power and intra-MNE competition are organizational level variables, I have used perception-based measures as they are considered to be more appropriate for research on human behaviour (Michailova \& Minbaeva, 2012).

Some limitations are related to sampling and sample selection. Due to difficulty in accessing respondents who work as engineers/researchers/scientists in a R\&D, I had to adopt a nonprobability sampling approach to select them. Also, to make data collection more feasible, information on knowledge transfer was collected from one side of the knowledge exchange relationship. Considering the dyadic characteristic of the variable, it would have been ideal to measure it at both sides, knowledge seeker and sharer, of the dyad (Mäkelä et al., 2012).

Another limitation of this study is that it used data sourced from single informant. Data sourced from a single informant may be susceptible to systematic bias and random errors, due to 
which the observed values may deviate from true values (Kumar, Stern, \& Anderson, 1993). It is suggested that multiple informants should be used for organizational studies (Wagner, Rau, \& Lindemann, 2010). But, studies undertaken in developing countries have to largely rely on a single informant due to constraints related to time and costs (Enticott, 2004). Moreover, the very nature of the data itself made it difficult to use multiple informants. The survey required the respondents to report their personal knowledge transfer behaviour along with information about the contacts with whom they engaged in knowledge transfers. Using multiple informants would have added to the complexity of data collection. Also, the data was cross-sectional. Data on subsidiary power is collected for the previous 2 years from the survey year. Ideally data on knowledge transfer should be collected for post survey years, say 2 years after the year of survey. However, time constraints did not allow such a longitudinal study.

Further, one more limitation of this study is that the investigation of knowledge transfer between subsidiaries is limited to the individual level. Individual knowledge transfers are considered to be the micro-foundations of organizational level transfers. Therefore, examining and establishing the link between individual and organizational transfers would have contributed and added greater value to the discourse on knowledge transfer at the organizational level. It is unfortunate that due to the availability of limited time and resources, it was beyond the scope of this study to examine how the assimilation of individual knowledge transfers lead to knowledge transfers at the organizational level.

\subsection{Future Research Avenues}

My study has opened up some research avenues that are worth pursuing in the future. It is recommended that future studies should consider testing this model by overcoming the limitations listed above.

\subsubsection{Multilevel analysis}

As revealed by the review of literature, the process of knowledge transfer and the factors that influence it in an MNC are of multilevel nature. Individuals, the primary actors in the process, are nested in subunits of organizations, which are again nested in multiple country contexts characterized by cultural, economic, political and geographic differences. Therefore, it is recommended that future studies should try to take into account the multilevel nested structure of the variables considered for research. Multilevel data collection and analysis is complex and 
challenging. So, it is suggested that before adopting a multilevel approach, the researcher should plan in advance and assess the feasibility of the study with respect to available time, resources, and data. Most importantly, it is advised that data collection should be planned well in advance to meet the requirements of a multilevel study. As put by Bassiri (1988), to have an adequate power 0.90 of the Hierarchical Linear Modelling to detect cross-level interactions, a sample of 30 groups with 30 individuals is necessary. It is preferable to collect data from more groups instead of many observations per group (Hofmann, 1997).

\subsubsection{Other cultural contexts}

Testing this model in a different country context, especially in a developed country culturally different from India will provide some interesting insights for comparison. It will help understand influences of different cultural dimensions on employee knowledge transfer behaviour. Using different cultural dimensions such as collectivism/individualism; power distance; long term/short term orientation etc. in the model will provide in-depth insights to the influence of culture. Also, investigating employee knowledge exchanges with contacts from subsidiaries which are relatively equal in power, say, between two developed country subsidiaries, may result in some fascinating outcomes. Also, it is motivating to test the model in a context similar to that of India as it would help validate the findings from India and discover new avenues for exploration.

Moreover, future research can explore the uncharted territory of multi-cultures in India which still remains greatly unexplored. Indian culture can broadly be divided into nine different subcultures (Dheer, Lenartowicz, \& Peterson, 2015). Exploring this cultural aspect of India would add valuable insights to understanding behaviours of employees coming from diverse cultures in India.

\subsubsection{Interaction of different mechanisms}

With respect to the variables used in this study, different facets of cultural intelligence and types of organizational identification, employees simultaneously identifying to various entities at different levels, there is ample room for exploration. It would be interesting to explore how identity based quasi-formal organizational KGA mechanisms interact with other formal or informal and individual or organizational level mechanisms to influence individual and organizational level knowledge transfers in the organization. 
Studying human behaviour opens a wider range of theories in psychology and social psychology, which can be used to explain employee behaviour in the organization. Insights from personality characteristics will add better understanding to examination of individual behaviour. Moreover, international business theories which are part and parcel of MNE operations also play an important role. The very crux of MNE decision making and control are the individual actors. An individual's decision making is influenced by factors both at the micro and macro organizational or country level. This makes the study of individual employee behaviour complex and challenging, but interesting at the same time.

\subsubsection{Study of knowledge transfers}

Looking into the pattern of knowledge exchanges in this study, as in Figure 6.2, it is observed that out of the respondents, a majority of them received knowledge mainly from the USA and Germany, followed by France, Sweden, and Netherlands. Whereas, with respect to sharing of knowledge, the main countries are USA, Germany, France, within India, China, and Korea.

These country specific patterns provide thought-provoking observations which can be explored in the future. A comparison of individual interactions of Indian employees with contacts from different countries is an area for future studies. Also, future studies can look into some objective measures of knowledge transfers by examining patents generated by the MNEs, from different countries and having the list of inventors belonging to different subsidiaries located in different countries. 
Figure 6.2 Countries from which knowledge is received and with which it is shared by the Indian subsidiary
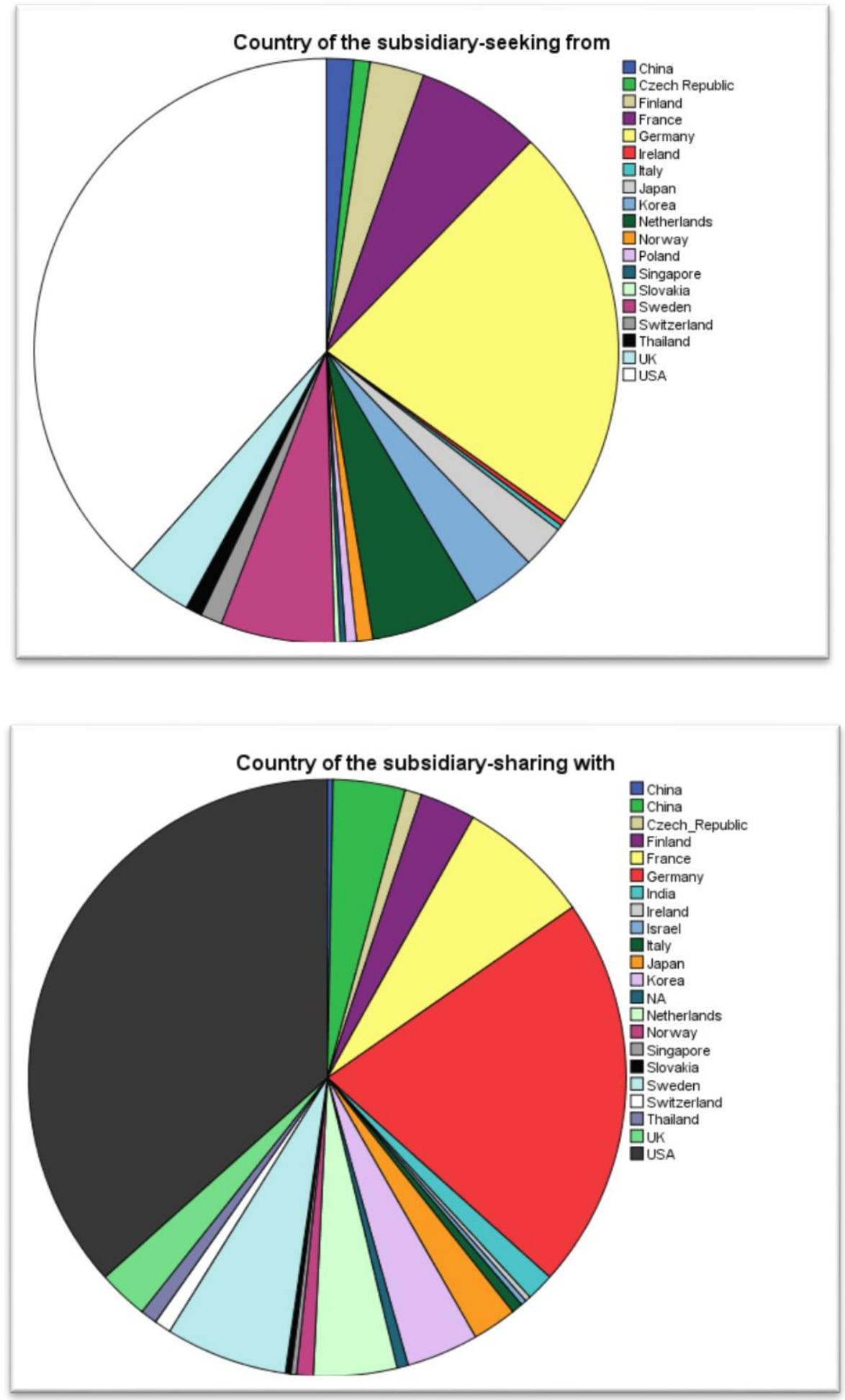
Although very ambitious, a future study can identify the individual inventors from particular MNEs located in different countries and undertake a study to investigate individual employee knowledge transfer behaviour. A mixed research methodology can be adopted by beginning with a qualitative exploration followed by quantitative analysis of the observed entities of prominence. This will provide a more robust examination of the knowledge transfer processes which have resulted in innovations, the tangible outcomes of knowledge transfer. Even, with this current research, I can examine patents and the list of inventors, generated by these MNEs in the future, and then explore a link to the observed knowledge transfer activities undertaken by the respondents of my study. This would provide better insights to the knowledge transfer process and its outcomes. This model can be extended and tested on other types of multinationals such as M\&As or alliances. Moreover, future research can examine the model in modern and non-traditional multinational structures such as the 'Global Factory System' (Buckley, 2011; Enderwick \& Buckley, 2019). In a global factory system, innovation is a collaborative venture as innovative assets may be possessed by partners who are external to the organization. The dynamics of power and competition as well as the relationships proposed in this study are expected to be different in these new forms of MNE structures than in MNEs with wholly owned subsidiaries.

\subsection{Conclusion}

Studying knowledge transfer in the MNE is of vital importance as it is the keystone to an MNE's success. From the review of literature it was revealed that it is a multidimensional, multidirectional, and multilevel process. It's antecedents are both at the organizational and individual levels. Individuals are the main actors in organizational knowledge transfers. The factors that influence individual knowledge transfer across subsidiaries are different from those that impact within subsidiary knowledge exchanges. Due to organizational separation of the MNE, and the dynamics of power and competition that exists amongst the subsidiaries, it was proposed that subsidiary power, intra-MNE competition, and cultural intelligence of the employees would influence knowledge transfers across subsidiaries. Drawing insights from the Knowledge Based View and Social Identity Theory, a model was proposed and hypotheses were developed. To test the model, data was collected from employees working in Indian subsidiaries of MNEs. Mixed results were found for the hypotheses tests from the data analysis. As predicted, subsidiary power, organizational identification, intra-MNE competition, and cultural intelligence were found to have influence, whether direct or indirect, on individual knowledge transfers. Therefore, it is proposed 
that these factors may be used as governance mechanisms to manage individual knowledge transfers. Subsidiary power and intra-MNE competition are identified as quasi-formal identity based mechanisms which can be used in organizations to govern knowledge exchanges. In addition to contributing to the Knowledge Governance Approach, this study advances our knowledge of organizational knowledge transfer processes, applications of social identity theory in organizations and towards understanding employee behaviour in the context of India. Thus, the findings from the study are used to put forward recommendations to managers. Like any other study, this study has some limitations which need to be overcome in future studies. In conclusion, this study opens up new avenues for research which are worth exploring in the future. 


\section{Appendices}

\section{Appendix 1: Questionnaire}

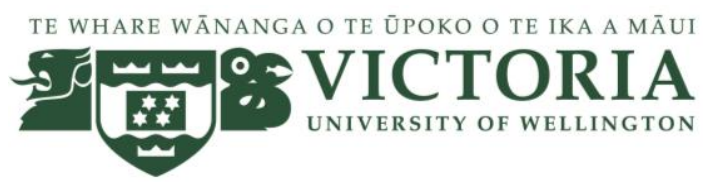

\section{WELCOME}

Thank you for participating in this survey. The survey is about interpersonal cross border knowledge exchange behaviour of subsidiary professionals. The questionnaire should be completed by a subsidiary employee who is engaged in knowledge exchange with employees within the MNE from other subsidiaries located in countries other than India.

The survey takes about 10 to 12 minutes to complete. Please note that the survey is strictly confidential to the research team. Your answers will not be shared with anyone either within your organization or outside it. There are no right or wrong answers, therefore please answer each question as completely and accurately as you can.

This project is administered by Himadree Phookan (PhD Scholar) under the supervision of Dr Revti Raman and Dr Cheryl Rivers, Victoria University of Wellington, Wellington, New Zealand.

\section{Section A}

\section{This section deals with interpersonal cross border knowledge transfer: knowledge INFLOWS}

Please identify a contact in the MNE from a subsidiary outside the country from whom you sought most of information or know how related to your work informally during the last 12 months-Information related to data, documents, etc. and know-how such as advice on how to deal with work-related problem, personal insight, tricks of the trade, etc.

This is the contact of your choice. The organization has not formally instructed you to seek know-how/information from this contact as per the prescribed path of a particular task. This is the contact you choose to go to out of various other available sources of information across different subsidiaries when in need of valuable advice/information/know-how relevant to work. Please exclude direct bosses or subordinates.

The medium of communication might have been through email, telephone, video-conferencing or any other communication mechanisms; or through face- to- face contact in conferences or meetings.

* Please provide the following demographics of your identified contact

1.1 Contact's age: $\square$ 20-29 $\square$ 30-39 $\square$ 40-49 $\square$ 50-59 $\square 60$ and above

1.2 Contact's gender: M / F / Other

1.3 Location/name of the subsidiary the contact belongs to (city, country)

1.4 How long has the contact been working in the current organization? (If known).

* Please keep the identified contact in mind and rate the following statements about the amount of knowledge/information you have received from this contact

During the last 12 months I have received from the identified contact

$(1=$ Very little, $2=$ Little, $3=$ Neither little nor much, $4=$ Much, $5=$ Very much $)$ 


$\begin{array}{lllllll}1.5 & \text { facts or information } & 1 & 2 & 3 & 4 & 5 \\ 1.6 & \text { advice to deal with work-related problem } & 1 & 2 & 3 & 4 & 5 \\ 1.7 & \text { personal insights about work } & 1 & 2 & 3 & 4 & 5 \\ 1.8 & \text { tricks of the trade } & 1 & 2 & 3 & 4 & 5\end{array}$

\section{* Please rate the usefulness of knowledge/information you have received from this contact}

I find...... $\quad(1=$ Not at all useful, 2 = Slightly useful, 3 = Useful, 4=Very useful, 5=Extremely useful $)$

$\begin{array}{lllllll}1.9 & \text { facts or information } & 1 & 2 & 3 & 4 & 5 \\ 1.10 & \text { advice to deal with work-related problem } & 1 & 2 & 3 & 4 & 5 \\ 1.11 & \text { personal insights about work } & 1 & 2 & 3 & 4 & 5 \\ 1.12 & \text { tricks of the trade } & 1 & 2 & 3 & 4 & 5\end{array}$

* Was it possible for you to accomplish the task for which you sought advice, without information or materials from this person? Please tick as appropriate
$1.13 \square$ 1=not at all possible $\square$ 2=less possible $\square$ 3=somewhat possible $\square$ 4=much possible $\square 5$ =very much possible

* Please rank your subsidiary and the contact's subsidiary regarding how much power they have within the multinational. Power is the subsidiary's ability to influence HQ's decisions and its ability to take decisions autonomously with less control from the HQ.

$$
\text { (1= Very little, } 2 \text { = little, } 3 \text { =Neither little nor much, } 4=\text { Much, 5= Very much) }
$$

Ability of the subsidiaries (your subsidiary \& the contact's subsidiary) to influence HQ's decisions

$\begin{array}{llllllll}1.14 & \text { Your subsidiary } & 1 & 2 & 3 & 4 & 5 \\ 1.15 & \text { Contact's subsidiary } & 1 & 2 & 3 & 4 & 5\end{array}$

Ability of the subsidiaries (your subsidiary \& the contact's subsidiary) to take decisions autonomously with less control from the HQ

$\begin{array}{lllllll} & & 1 & 2 & 3 & 4 & 5 \\ 1.16 & \text { Your subsidiary } & & & & & \\ & \text { Contact subsidiary } & 1 & 2 & 3 & 4 & \end{array}$

- How would you describe the competitive nature between your subsidiary and this contact's subsidiary? Your response should be based on your assessment for the last year. Please tick as appropriate.

(1= No competition, $2=$ Low competition, $3=$ Moderate competition,

1.18 Competition with respect to funding/resources from HQ 4= High competition, 5= Very high competition) 


$\begin{array}{ll}1.19 & \begin{array}{l}\text { Competition for markets (sell products to the same external } \\ \text { market) }\end{array} \\ 1.20 & \begin{array}{l}\text { Competition due to the subsidiary producing similar types of } \\ \text { products and technologies as yours. }\end{array}\end{array}$

\footnotetext{
* Please select one of the following choices regarding the relationship that you share with the contact

$1.21 \square$ You have known the contact for years. You trust him/her and consider him/her a friend. He/She belongs to your social network.

$\square$ You know the contact in passing; consider him/her an acquaintance and not really a part of your social network
}

\section{Section B}

\section{This section deals with interpersonal cross border knowledge transfer: knowledge OUTFLOWS}

Please identify a contact in the MNE from a subsidiary outside the country who sought most of information or know how related to his/her work from you informally during the last 12 months- Information related to data, documents, etc. and know-how such as advice on how to deal with work-related problem, personal insight, tricks of the trade, etc.

This is the contact that chose to come to you out of various other available sources of information across different subsidiaries when in need of valuable advice/information/know-how relevant to work. The organization has not formally instructed him/her to seek know-how/information from you as per the prescribed path of a particular task. Please exclude direct bosses or subordinates.

The medium of communication might have been through email, telephone, video-conferencing or any other communication mechanisms; or through face- to- face contact in conferences or meetings.

\section{* Is this contact the same person from whom you sought knowledge as identified in section A?}

Yes. Please skip the following questions from 1.22 to 1.25 and go to 1.26

No. Please continue with the following questions.

* Please provide the following demographics of your identified contact

1.22 Contact's age: $\square 20-29 \quad \square$ 30-39 $\square$ 40-49 $\square$ 50-59 $\square 60$ and above

1.23 Contact's gender: $\mathrm{M} / \mathrm{F}$ / other

1.24 Location/name of the subsidiary the contact belongs to (city, country)

1.25 How long has the contact been working in the current organization? (If known)

\section{* Please keep the identified contact in mind and rate the following statements about the amount of knowledge/information you have shared with this contact.}

During the last 12 months I have shared with the identified contact

(1=Very little, 2 = Little, 3 = Neither little nor much, 4= Much, 5= Vey much)
1.26 facts or information
1.27 advice to deal with work-related problem
1.28 personal insights about work

$\begin{array}{lllll}1 & 2 & 3 & 4 & 5 \\ 1 & 2 & 3 & 4 & 5 \\ 1 & 2 & 3 & 4 & 5\end{array}$


1.29 tricks of the trade

$\begin{array}{llll}1 & 2 & 3 & 4\end{array}$

5

* Please rate the usefulness of knowledge/information you have shared with this contact

I find..... (1=Not at all useful, 2 =Slightly useful, $3=$ Useful, 4=Very useful, 5=Extremely useful)

1.30 facts or information $\quad 1 \quad 1 \quad \begin{array}{lllll}2 & 3 & 4 & 5\end{array}$

1.31 advice to deal with work-related problem $\quad \begin{array}{lllllll}1 & 2 & 3 & 4 & 5\end{array}$

1.32 personal insights about work $\quad \begin{array}{llllll}1 & 2 & 3 & 4 & 5\end{array}$

1.33 tricks of the trade $\quad \begin{array}{lllllll}1 & 2 & 3 & 4 & 5\end{array}$

* Was it possible for him/her to accomplish the task for which he/she sought advice from you, without information or materials from you? Please tick as appropriate

1.34 $\square$ 1=not at all possible $\square$ 2=less possible $\square$ 3=somewhat possible $\square$ 4=much possible $\square$ =very much possible

* Is this contact the same person from whom you sought knowledge as identified in section A?/Or, does the contact belong to the same subsidiary as the contact identified in section $\mathrm{A}$ ?

Yes. Please skip the following questions from 1.35 to 1.39 and go to 1.40 .

No. Please continue with the following questions.

* Please rank the contact's subsidiary regarding how much power it has within the multinational. Power is the subsidiary's ability to influence HQ's decisions and its ability to take decisions autonomously with less control from the HQ.

(1=Very little, 2 =Little, 3 =Neither little nor much, $4=$ Much, 5=Very much)

1.35 Ability of the subsidiary to influence HQ's decisions

1.36 Ability of the subsidiary to take decisions autonomously with less control from the HQ

* How would you describe the competitive nature between your subsidiary and this contact's subsidiary? Your response should be based on your assessment for the last year. Please tick as appropriate.

( $1=$ No competition, $2=$ Low competition, $3=$ Moderate competition, 4=High competition, $5=$ Very high competition)

1.37 Competition with respect to funding/resources from HQ

1.38 Competition for markets (sell products to the same external market)

$\begin{array}{lllllllll}1.39 & \text { Competition due to the subsidiary producing similar types of } & 1 & 2 & 3 & 4 & 5\end{array}$ products and technologies as yours

* Please select one of the following choices regarding the relationship that the contact shares with you

$1.40 \square \mathrm{He} / \mathrm{She}$ has known you for years. He/She trusts you and considers you a friend. You belong to his/her social network.

$\square$ He/She knows you in passing; considers you an acquaintance and not really a part of his/her social network 


\section{Section C}

\section{This section helps understanding you and your subsidiary further}

\section{* Please rate the following about your subsidiary}

(1=Strongly disagree, 2 = Disagree, $3=$ Neither agree nor disagree, 4=Agree, $5=$ Strongly agree)

1.41 The practices of this local unit/subsidiary are in line with my $\quad \begin{array}{lllll}1 & 2 & 3 & 4 & 5\end{array}$ personal values

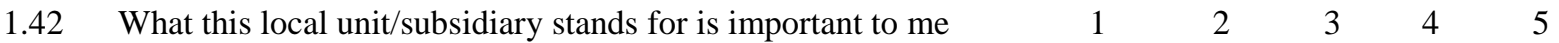

1.43 My values and the values of the local unit/subsidiary that I work $\quad \begin{array}{llllll}1 & 2 & 3 & 4 & 5\end{array}$ for are the same

1.44 I am proud to tell others that I am an employee of this company $\quad \begin{array}{lllll}1 & 2 & 3 & 4\end{array}$

1.45 In this company we have capable and sensible people in top $\quad \begin{array}{lllll}1 & 2 & 3 & 4 & 5\end{array}$ management

1.46 This company is likely to be successful in the future $\quad 1 \quad 2 \quad 3 \quad 4 \quad 5$

* Please indicate to what extent each of the following statements truly describes you. These statements are about your experience when interacting with people from other cultures.

(1 = Not at all, $2=$ A little, $3=$ Somewhat, $4=$ A lot, $5=$ Extremely well $)$

1.47 I know the ways in which cultures around the world are different. $\quad \begin{array}{llllll}1 & 2 & 3 & 4\end{array}$

1.48 I can give examples of cultural differences from my personal $\quad \begin{array}{lllll}1 & 2 & 3 & 4\end{array}$ experience, reading, and so on.

1.49 I enjoy talking with people from different cultures.

1.50 I have the ability to accurately understand the feelings of people from other cultures.

1.51 I sometimes try to understand people from another culture by imagining how something looks from their perspective.

1.52 I can change my behaviour to suit different cultural situations and people.

1.53 I accept delays without becoming upset when in different cultural situations and with culturally different people.

1.54 I am aware of the cultural knowledge I use when interacting with $\quad \begin{array}{lllll}1 & 2 & 3 & 4\end{array}$ someone from another culture.

1.55 I think a lot about the influence that culture has on my behaviour $\quad \begin{array}{llll}1 & 2 & 3 & 4\end{array}$ and that of others who are culturally different.

1.56 I am aware that I need to plan my course of action when in different cultural situations and with culturally different people. 
* Please rate your fashion consciousness. How important is it for you to dress fashionably?

( 1 = strongly disagree, 2 = somewhat disagree, 3 = neither agree nor disagree, 4 = somewhat agree, $5=$ strongly agree)

1.57 When I must choose between the two, I usually dress for fashion, not for comfort

1.58 An important part of my life and activities is dressing smartly

1.59 A person should try to dress in style

$\begin{array}{lllll}1 & 2 & 3 & 4 & 5 \\ 1 & 2 & 3 & 4 & 5 \\ 1 & 2 & 3 & 4 & 5\end{array}$

\section{Section D}

This section helps understanding demography of the respondent (you)

1.60 Your gender: M / F / Other

1.61 Your age in years: $\square$ 20-29 $\square 30-39 \quad \square$ 40-49 $\square 50-59 \quad \square 60$ and above

1.62 Your highest degree:

1.63 How long have you been working in this organisation (Years) .........

1.64 Your nationality: Your home state, if Indian National:

1.65 What is your first language:

Please provide your contact details if you would like to receive an executive summary of the findings. (Optional)

Your name:

Position:

Email ID:

Or, You may email at himadree.phookan@vuw.ac.nz to get the executive summary of findings.

THANK YOU 


\section{Appendix 2: Participant information sheet}

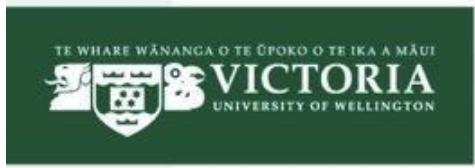

SCHOOL OF MARKETING AND INTERNATIONAL BUSINESS

TE KURA HOKOHOKO, PAKIHI KI TE AO

LEVEL 11, RUTHERFORD HOUSE, PIPITEA CAMPUS, 23 LAMBTON QUAY, WELLINGTON

PO Box 600 , Wellington 6140 , New Zealand

Phone+64-4-4635330 Fax +64-4-4635231 Email: marketing@vuw.ac.nz Website: www.victoria.ac.nz/smib

\section{Participant Information Sheet}

Thank you for your interest in this study.

My name is Himadree Phookan, a doctoral student in the program, PhD in International Business at Victoria University of Wellington, New Zealand. This study is about my thesis "Subsidiary Power and intra-MNE interpersonal cross-border Knowledge Transfer: The Role of Intra-MNE Competition and Strategic International HRM management systems".

This study aims at identifying the factors which may create barrier or facilitate the process of knowledge exchange between subsidiary employees across different countries. This survey is voluntary and the information confidential. This research has been approved by the Victoria University of Wellington Human Ethics Committee, reference: 0000024345 . Should you feel the need to withdraw from the project, you may do so at any time before $1^{\text {st }}$ of April, 2018. Please let me know if you decide to withdraw.

Your organization's participation will be through a questionnaire survey addressed to managers and employees in the subsidiary unit. The survey questionnaire for the employees would take approximately $10 \mathrm{~min}$ to complete. All the material related to the survey responses will be viewed only by the researcher and the research supervisor. It will not be shared with anyone else-within your organization or outside it. Your responses will be confidential to the researcher and the research supervisor- you or your firm will not be identified in any way by anyone except them. All electronic data, if any, will be kept in a password protected file accessible only to the researcher. Data collected in this survey will be destroyed/deleted after 5 years. It is possible that the summary results from this survey may appear in academic or professional journals and may also be presented at academic or professional conferences. I am happy to share the executive summary of my findings with you on successful completion of the project.

Your participation in the survey is very important for effective completion of this research. This will immensely contribute towards understanding key issues in knowledge transfer across borders within the multinational.

If you have any concerns, questions, or require any further information, please feel free to contact me.

Himadree Phookan

PhD Scholar

Himadree.phookan@vuw.ac.nz

\section{Research Supervisor}

Dr. Revti Raman,

Senior Lecturer,

School of Marketing and International Business,

Victoria University of Wellington, New Zealand

Revti.raman@vuw.ac.nz

\section{For ethics queries}

AProf Susan Corbett

Victoria University of Wellington, New Zealand

Susan.corbett@vuw.ac.nz

Phone: +64-4-463 5480 


\section{Appendix 3 Missing values \& response bias}

Table 1 Missing Values

\begin{tabular}{|c|c|c|}
\hline \multicolumn{3}{|l|}{ Observed variables } \\
\hline \multirow{2}{*}{ Q1.1 Age of the contact-seeking } & Valid & 329 \\
\hline & Missing & 4 \\
\hline \multirow{2}{*}{ Q1.4 Tenure of the contact-seeking } & Valid & 269 \\
\hline & Missing & 64 \\
\hline \multirow{2}{*}{ Q1.5 Facts-Information-seeking } & Valid & 333 \\
\hline & Missing & 0 \\
\hline \multirow{2}{*}{ Q1.6 Advice-seeking } & Valid & 333 \\
\hline & Missing & 0 \\
\hline \multirow{2}{*}{ Q1.7 Personal insights-seeking } & Valid & 333 \\
\hline & Missing & 0 \\
\hline \multirow{2}{*}{ Q1.8 Tricks-seeking } & Valid & 333 \\
\hline & Missing & 0 \\
\hline \multirow{2}{*}{ Q1.9 Facts-Information useful-seeking } & Valid & 333 \\
\hline & Missing & 0 \\
\hline \multirow{2}{*}{ Q1.10 Advice useful-seeking } & Valid & 333 \\
\hline & Missing & 0 \\
\hline \multirow{2}{*}{ Q1.11 Personal insights useful-seeking } & Valid & 333 \\
\hline & Missing & 0 \\
\hline \multirow{2}{*}{ Q1.12 Tricks useful-seeking } & Valid & 333 \\
\hline & Missing & 0 \\
\hline \multirow{2}{*}{ Q1.13 Task interdependency-seeking } & Valid & 333 \\
\hline & Missing & 0 \\
\hline \multirow{2}{*}{ Q1.14 Influence on HQ-own subsidiary } & Valid & 333 \\
\hline & Missing & 0 \\
\hline \multirow{2}{*}{ Q1.15 Influence-contact subsidiary-seeking } & Valid & 333 \\
\hline & Missing & 0 \\
\hline \multirow{2}{*}{ Q1.16 Autonomy-own subsidiary } & Valid & 333 \\
\hline & Missing & 0 \\
\hline \multirow{2}{*}{ Q1.17 Autonomy-contact subsidiary-seeking } & Valid & 333 \\
\hline & Missing & 0 \\
\hline \multirow{2}{*}{ Q1.18 Competition for funding-seeking } & Valid & 333 \\
\hline & Missing & 0 \\
\hline \multirow{2}{*}{ Q1.19 Competition for markets-seeking } & Valid & 333 \\
\hline & Missing & 0 \\
\hline \multirow{2}{*}{ Q1.20 Competition similar products-seeking } & Valid & 333 \\
\hline & Missing & 0 \\
\hline \multirow{2}{*}{ Q1.22 Age of the contact-sharing } & Valid & 322 \\
\hline & Missing & 11 \\
\hline \multirow{2}{*}{ Q1.25 Tenure of the contact-sharing } & Valid & 244 \\
\hline & Missing & 89 \\
\hline \multirow{2}{*}{ Q1.26 Facts-Information-sharing } & Valid & 333 \\
\hline & Missing & 0 \\
\hline O1 27 Advice-sharino & Valid & 333 \\
\hline & Missing & 0 \\
\hline O1 28 Perconal insiohts-sharino & Valid & 333 \\
\hline Q 1.28 Personal insights-sharıng & Missing & 0 \\
\hline Q1 20 Trioke shorino & Valid & 333 \\
\hline Q 1.29 1IICKs-snarmg & Missing & 0 \\
\hline Q1 30 Eoctc Information uceful charino & Valid & 333 \\
\hline Q1.30 Facts-Information useful-sharıng & Missing & 0 \\
\hline
\end{tabular}




\begin{tabular}{|c|c|c|}
\hline Q1.31 Advice useful-sharing & $\begin{array}{c}\text { Valid } \\
\text { Missing }\end{array}$ & $\begin{array}{c}333 \\
0\end{array}$ \\
\hline Q1.32 Personal insights useful-sharing & $\begin{array}{c}\text { Valid } \\
\text { Missing }\end{array}$ & $\begin{array}{c}333 \\
0\end{array}$ \\
\hline Q1.33 Tricks useful-sharing & $\begin{array}{c}\text { Valid } \\
\text { Missing }\end{array}$ & $\begin{array}{c}333 \\
0\end{array}$ \\
\hline Q1.34 Task interdependency-sharing & $\begin{array}{c}\text { Valid } \\
\text { Missing }\end{array}$ & $\begin{array}{c}327 \\
6 \\
\end{array}$ \\
\hline Q1.35 Influence-contact subsidiary-sharing & $\begin{array}{c}\text { Valid } \\
\text { Missing }\end{array}$ & $\begin{array}{c}333 \\
0\end{array}$ \\
\hline Q1.36 Autonomy-contact subsidiary-sharing & $\begin{array}{c}\text { Valid } \\
\text { Missing }\end{array}$ & $\begin{array}{c}333 \\
0\end{array}$ \\
\hline Q1.37 Competition for funding-sharing & $\begin{array}{c}\text { Valid } \\
\text { Missing }\end{array}$ & $\begin{array}{c}333 \\
0\end{array}$ \\
\hline Q1.38 Competition for markets-sharing & $\begin{array}{c}\text { Valid } \\
\text { Missing }\end{array}$ & $\begin{array}{c}333 \\
0 \\
\end{array}$ \\
\hline Q1.39 Competition similar products-sharing & $\begin{array}{c}\text { Valid } \\
\text { Missing }\end{array}$ & $\begin{array}{c}333 \\
0\end{array}$ \\
\hline Q1.41 Identification-practices & $\begin{array}{c}\text { Valid } \\
\text { Missing }\end{array}$ & $\begin{array}{c}333 \\
0\end{array}$ \\
\hline Q1.42 Identification-stands for & $\begin{array}{c}\text { Valid } \\
\text { Missing }\end{array}$ & $\begin{array}{c}333 \\
0 \\
\end{array}$ \\
\hline Q1.43 Identification-values & $\begin{array}{c}\text { Valid } \\
\text { Missing }\end{array}$ & $\begin{array}{c}333 \\
0\end{array}$ \\
\hline Q1.44 Identification-proud & $\begin{array}{c}\text { Valid } \\
\text { Missing }\end{array}$ & $\begin{array}{c}333 \\
0\end{array}$ \\
\hline Q1.45 Identification-capability & $\begin{array}{c}\text { Valid } \\
\text { Missing }\end{array}$ & $\begin{array}{c}333 \\
0\end{array}$ \\
\hline Q1.46 Identification-future success & $\begin{array}{c}\text { Valid } \\
\text { Missing }\end{array}$ & $\begin{array}{c}333 \\
0\end{array}$ \\
\hline Q1.47 Cultural Intelligence-culture diff & $\begin{array}{c}\text { Valid } \\
\text { Missing }\end{array}$ & $\begin{array}{c}333 \\
0\end{array}$ \\
\hline Q1.48 Cultural Intelligence-eg & $\begin{array}{c}\text { Valid } \\
\text { Missing }\end{array}$ & $\begin{array}{c}333 \\
0\end{array}$ \\
\hline Q1.49 Cultural Intelligence-enjoy talking & $\begin{array}{c}\text { Valid } \\
\text { Missing }\end{array}$ & $\begin{array}{c}333 \\
0 \\
\end{array}$ \\
\hline Q1.50 Cultural Intelligence-understand & $\begin{array}{c}\text { Valid } \\
\text { Missing }\end{array}$ & $\begin{array}{c}333 \\
0\end{array}$ \\
\hline Q1.51 Cultural Intelligence-their perspective & $\begin{array}{c}\text { Valid } \\
\text { Missing }\end{array}$ & $\begin{array}{c}333 \\
0\end{array}$ \\
\hline Q1.52 Cultural Intelligence-change behaviour & $\begin{array}{c}\text { Valid } \\
\text { Missing }\end{array}$ & $\begin{array}{c}333 \\
0\end{array}$ \\
\hline Q1.53 Cultural Intelligence-accept delays & $\begin{array}{c}\text { Valid } \\
\text { Missing }\end{array}$ & $\begin{array}{c}333 \\
0\end{array}$ \\
\hline Q1.54 Cultural Intelligence-use cultural know & $\begin{array}{c}\text { Valid } \\
\text { Missing }\end{array}$ & $\begin{array}{c}333 \\
0\end{array}$ \\
\hline Q1.55 Cultural Intelligence-influence behaviour & $\begin{array}{c}\text { Valid } \\
\text { Missing }\end{array}$ & $\begin{array}{c}333 \\
0\end{array}$ \\
\hline Q1.56 Cultural Intelligence-plan of action & $\begin{array}{c}\text { Valid } \\
\text { Missing }\end{array}$ & $\begin{array}{c}333 \\
0\end{array}$ \\
\hline Q1.57 MV Dress for fashion not comfort & $\begin{array}{c}\text { Valid } \\
\text { Missing }\end{array}$ & $\begin{array}{c}333 \\
0\end{array}$ \\
\hline
\end{tabular}




\begin{tabular}{|lcc|}
\hline Q1.58 MV Dressing smartly & Valid & 333 \\
& Missing & 0 \\
\hline \multirow{2}{*}{ Q1.59 MV Dress in style } & Valid & 333 \\
& Missing & 0 \\
\hline \multirow{2}{*}{ Q1.61 Respondent age } & Valid & 325 \\
& Missing & 8 \\
\hline \multirow{2}{*}{ Q1.62 Respondent qualification } & Valid & 321 \\
& Missing & 12 \\
\hline \multirow{2}{*}{ Q1.63 Respondent tenure } & Valid & 318 \\
& Missing & 15 \\
\hline
\end{tabular}

Table 2 Response bias knowledge seeking

\begin{tabular}{|c|c|c|c|c|c|}
\hline \multicolumn{6}{|c|}{ Group Statistics } \\
\hline & Groups & $\mathrm{N}$ & Mean & Std. Deviation & Std. Error Mean \\
\hline \multirow{2}{*}{ Facts-Information received } & Paper survey & 193 & 3.73 & .812 & .058 \\
\hline & Online survey & 140 & 3.69 & .759 & .064 \\
\hline \multirow{2}{*}{ Advice received } & Paper survey & 193 & 3.12 & .982 & .071 \\
\hline & Online survey & 140 & 2.96 & .913 & .077 \\
\hline \multirow{2}{*}{ Personal insights received } & Paper survey & 193 & 3.30 & .931 & .067 \\
\hline & Online survey & 140 & 3.31 & .856 & .072 \\
\hline \multirow{2}{*}{ Tricks received } & Paper survey & 193 & 2.78 & 1.014 & .073 \\
\hline & Online survey & 140 & 2.62 & .844 & .071 \\
\hline \multirow{2}{*}{ Facts-Information useful } & Paper survey & 193 & 3.67 & .765 & .055 \\
\hline & Online survey & 140 & 3.43 & .741 & .063 \\
\hline \multirow{2}{*}{ Advice useful } & Paper survey & 193 & 3.07 & .982 & .071 \\
\hline & Online survey & 140 & 2.77 & .843 & .071 \\
\hline \multirow{2}{*}{ Personal insights useful } & Paper survey & 193 & 3.12 & .925 & .067 \\
\hline & Online survey & 140 & 2.86 & .773 & .065 \\
\hline \multirow{2}{*}{ Tricks useful } & Paper survey & 193 & 2.91 & 1.009 & .073 \\
\hline & Online survey & 140 & 2.46 & .826 & .070 \\
\hline \multicolumn{6}{|c|}{$\begin{array}{l}\text { Independent Samples Test } \\
\text { t-test for equality of Means }\end{array}$} \\
\hline & & & $\mathrm{t}$ & df & Sig.(2-tailed) \\
\hline \multirow{2}{*}{ Facts-Information received } & Equal varianc & & 0.452 & 331.000 & 0.115 \\
\hline & Equal varianc & sumed & 0.457 & 311.436 & 0.110 \\
\hline \multirow{2}{*}{ Advice received } & Equal varianc & & 1.580 & 331.000 & 0.115 \\
\hline & Equal varianc & sumed & 1.598 & 313.135 & 0.110 \\
\hline \multirow{2}{*}{ Personal insights received } & Equal varianc & & -0.066 & 331.000 & 0.947 \\
\hline & Equal varianc & sumed & -0.067 & 324.768 & 0.946 \\
\hline \multirow{2}{*}{ Tricks received } & Equal varianc & & 1.482 & 331.000 & 0.139 \\
\hline & Equal varianc & sumed & 1.526 & 305.114 & 0.127 \\
\hline \multirow{2}{*}{ Facts-Information useful } & Equal varianc & hed & 2.922 & 331.000 & 0.003 \\
\hline & Equal varianc & sumed & 2.937 & 321.683 & 0.003 \\
\hline \multirow{2}{*}{ Advice useful } & Equal varianc & hed & 2.929 & 331.000 & 0.003 \\
\hline & Equal varianc & sumed & 3.001 & 321.683 & 0.002 \\
\hline \multirow{2}{*}{ Personal insights useful } & Equal varianc & hed & 2.730 & 331.000 & 0.006 \\
\hline & Equal varianc & ssumed & 2.808 & 324.315 & 0.005 \\
\hline \multirow{2}{*}{ Tricks useful } & Equal varianc & ned & 4.305 & 331.000 & 0.000 \\
\hline & Equal varianc & ssumed & 4.443 & 326.148 & 0.000 \\
\hline
\end{tabular}


Table 3 Response bias knowledge sharing

\begin{tabular}{|c|c|c|c|c|c|}
\hline \multicolumn{6}{|c|}{ Group Statistics } \\
\hline & Group & $\mathrm{N}$ & Mean & Std. Deviation & Std. Error Mean \\
\hline \multirow{2}{*}{ Facts-Information shared } & Paper survey & 193 & 3.874 & .773 & .055 \\
\hline & Online survey & 140 & 3.827 & .656 & .055 \\
\hline \multirow{2}{*}{ Advice shared } & Paper survey & 193 & 3.187 & 1.003 & .072 \\
\hline & Online survey & 140 & 2.994 & .869 & .073 \\
\hline \multirow{2}{*}{ Personal insights shared } & Paper survey & 193 & 3.301 & .925 & .066 \\
\hline & Online survey & 140 & 3.180 & .850 & .071 \\
\hline \multirow{2}{*}{ Tricks shared } & Paper survey & 193 & 2.817 & 1.114 & .080 \\
\hline & Online survey & 140 & 2.676 & .907 & .076 \\
\hline \multirow{2}{*}{ Facts-Information shared useful } & Paper survey & 193 & 3.727 & .749 & .053 \\
\hline & Online survey & 140 & 3.676 & .691 & .058 \\
\hline \multirow{2}{*}{ Advice shared useful } & Paper survey & 193 & 3.057 & .873 & .062 \\
\hline & Online survey & 140 & 3.000 & .864 & .073 \\
\hline \multirow{2}{*}{ Personal insights shared useful } & Paper survey & 193 & 3.057 & .908 & .065 \\
\hline & Online survey & 140 & 2.957 & .794 & .067 \\
\hline \multirow{2}{*}{ Tricks shared useful } & Paper survey & 193 & 2.680 & 1.00 & .072 \\
\hline & Online survey & 140 & 2.547 & .968 & .081 \\
\hline \multicolumn{6}{|c|}{$\begin{array}{l}\text { Independent Samples Test } \\
\text { t-test for equality of Means }\end{array}$} \\
\hline & & & $\mathrm{t}$ & df & Sig.(2-tailed) \\
\hline \multirow{2}{*}{ Facts-Information shared } & Equal varian & issumed & .577 & 331 & .564 \\
\hline & Equal varian & lot assumed & .593 & 322.962 & .554 \\
\hline \multirow{2}{*}{ Advice shared } & Equal varian & sssumed & 1.836 & 331 & .067 \\
\hline & Equal varian & tot assumed & 1.878 & 320.673 & .061 \\
\hline \multirow{2}{*}{ Personal insights shared } & Equal varian & sssumed & 1.223 & 331 & .222 \\
\hline & Equal varian & tot assumed & 1.239 & 313.141 & .216 \\
\hline \multirow{2}{*}{ Tricks shared } & Equal varian & sssumed & 1.226 & 331 & .221 \\
\hline & Equal varian & tot assumed & 1.266 & 326.586 & .206 \\
\hline \multirow{2}{*}{ Facts-Information shared useful } & Equal varian & issumed & .634 & 331 & .527 \\
\hline & Equal varian & tot assumed & .642 & 312.564 & .522 \\
\hline \multirow{2}{*}{ Advice shared useful } & Equal varian & sssumed & .591 & 331 & .555 \\
\hline & Equal varian & lot assumed & .592 & 301.258 & .554 \\
\hline \multirow{2}{*}{ Personal insights shared useful } & Equal varian & issumed & 1.043 & 331 & .298 \\
\hline & Equal varian & lot assumed & 1.066 & 319.545 & .287 \\
\hline \multirow{2}{*}{ Tricks shared useful } & Equal varian & sssumed & 1.205 & 331 & .229 \\
\hline & Equal varian & tot assumed & 1.213 & 306.474 & .226 \\
\hline
\end{tabular}




\section{Appendix 4: Confirmatory factor analysis}

Figure 1 First Order CFA: Model 1

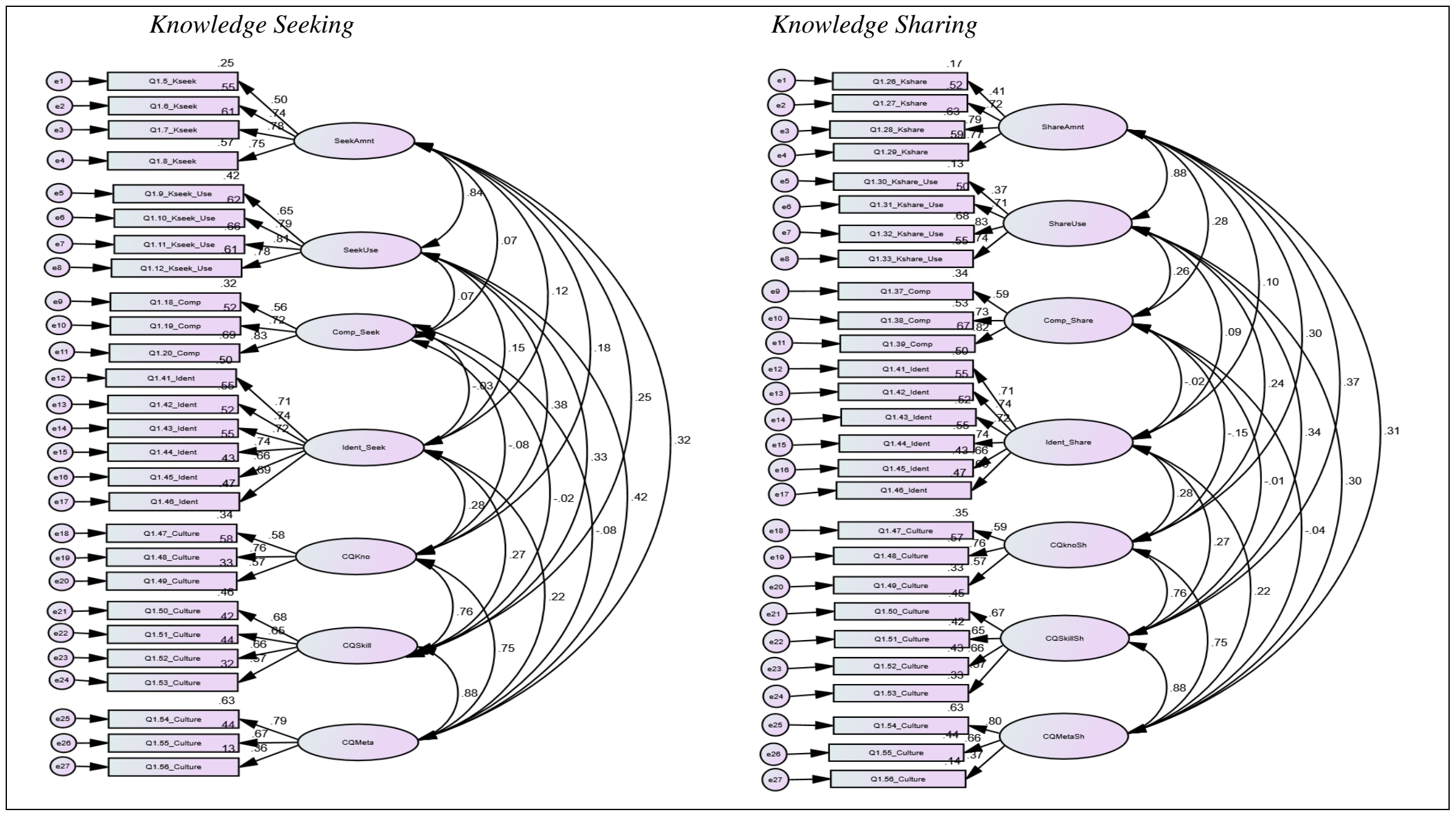


Figure 2 First Order CFA: Model 2

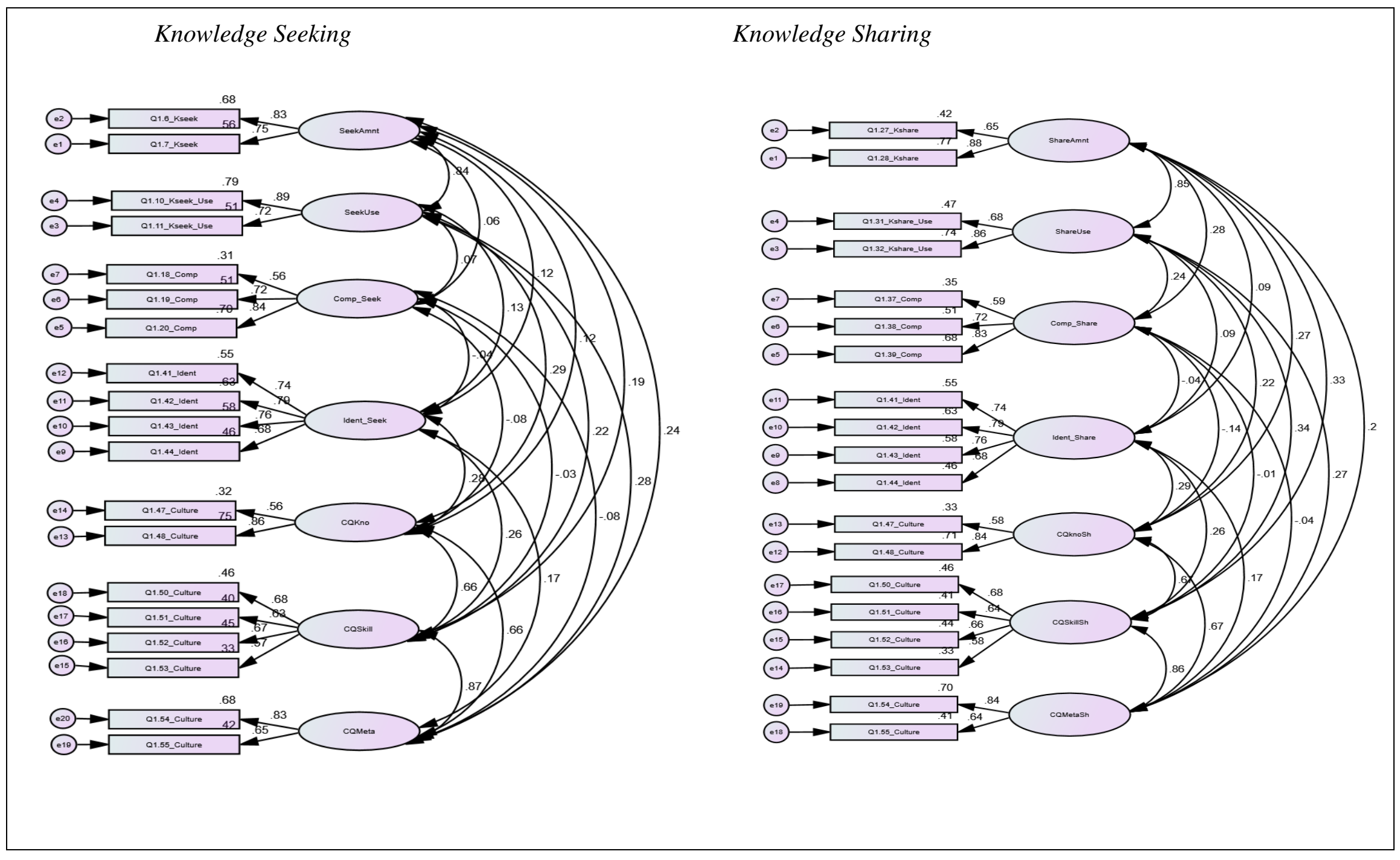




\section{Appendix 5: Linearity-scatter plots}

Figure 5a. The scatter plots of residuals of the combined independent variables on the dependent are below. A linear relationship is indicated in all the relationships.

1. Relationship between the IVsSubsidiary Power, Identification, Competition, $\mathrm{CQ}$, control variables and Knowledge Seeking producing the regression line.

$\mathrm{Y}=9.97 \mathrm{E}-17+1.53 \mathrm{E}-16 * \mathrm{x}$

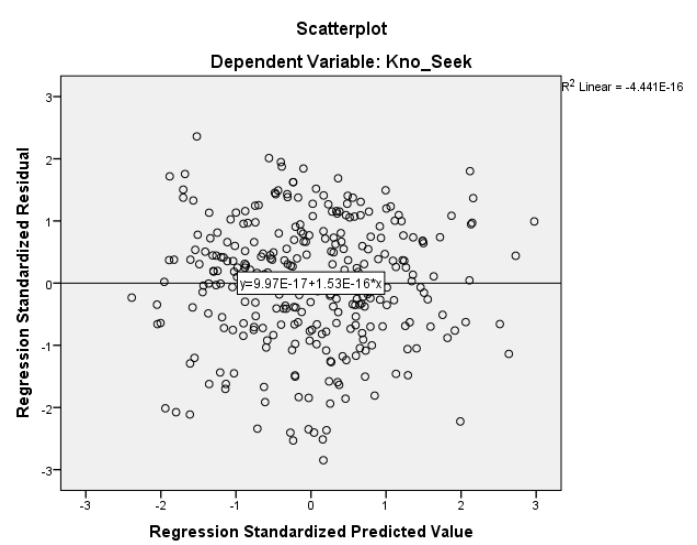

3. A linear relationship is indicated between the IVs- Subsidiary Power, control variables and Identification (for knowledge seeking model) producing the regression line $\mathrm{Y}=-$ 8.93E-17-3.13E-16*x

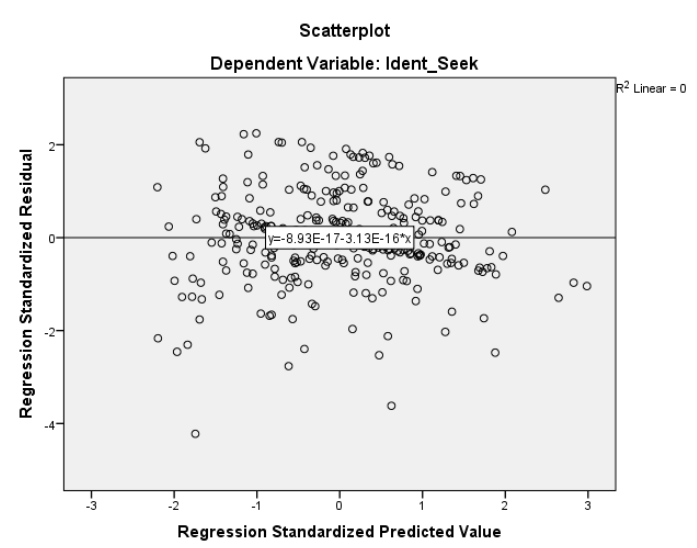

2. A liners relationship is indicated between the IVs- Subsidiary Power, Identification, Competition, CQ, control variables and Knowledge Sharing producing the regression line $\mathrm{Y}=-6.33 \mathrm{E}-17+1.04 \mathrm{E}-16 * \mathrm{x}$

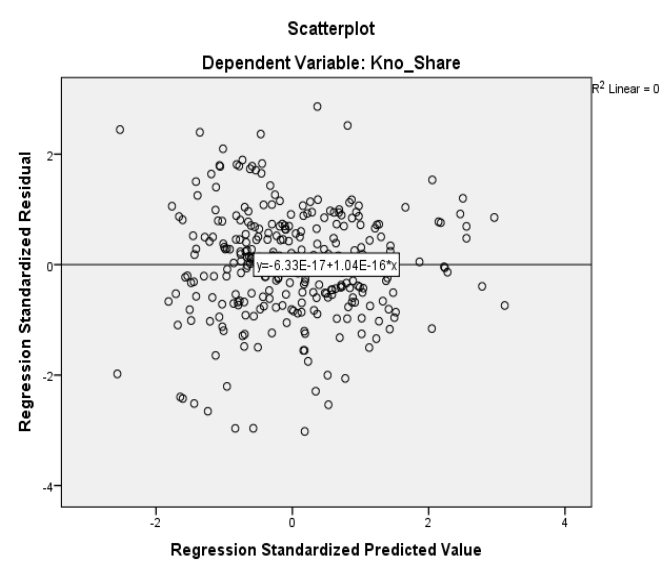

4. A linear relationship is indicated between the IVs- Subsidiary Power, control variables and Identification (for knowledge sharing model) producing the regression line $\mathrm{y}=2.86 \mathrm{E}-17-1.8 \mathrm{E}-17^{*} \mathrm{x}$

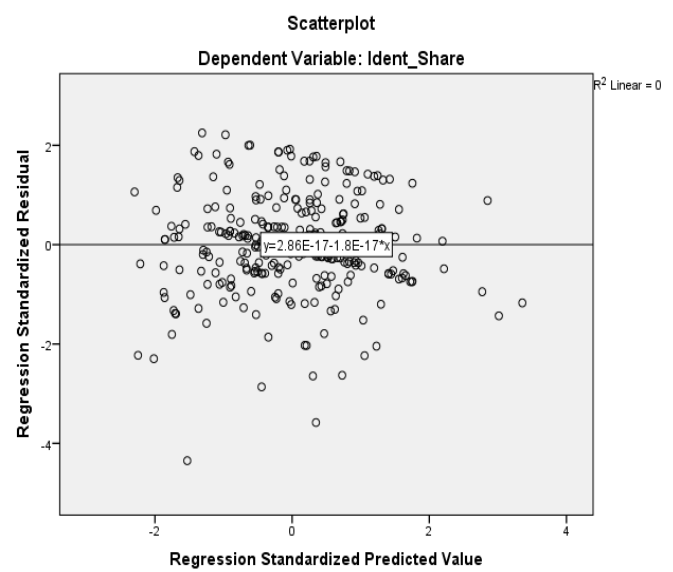


Figure $5 \mathrm{~b}$. The partial plots of each independent variable with the dependent variable are presented below.

Knowledge seeking with each independent variables
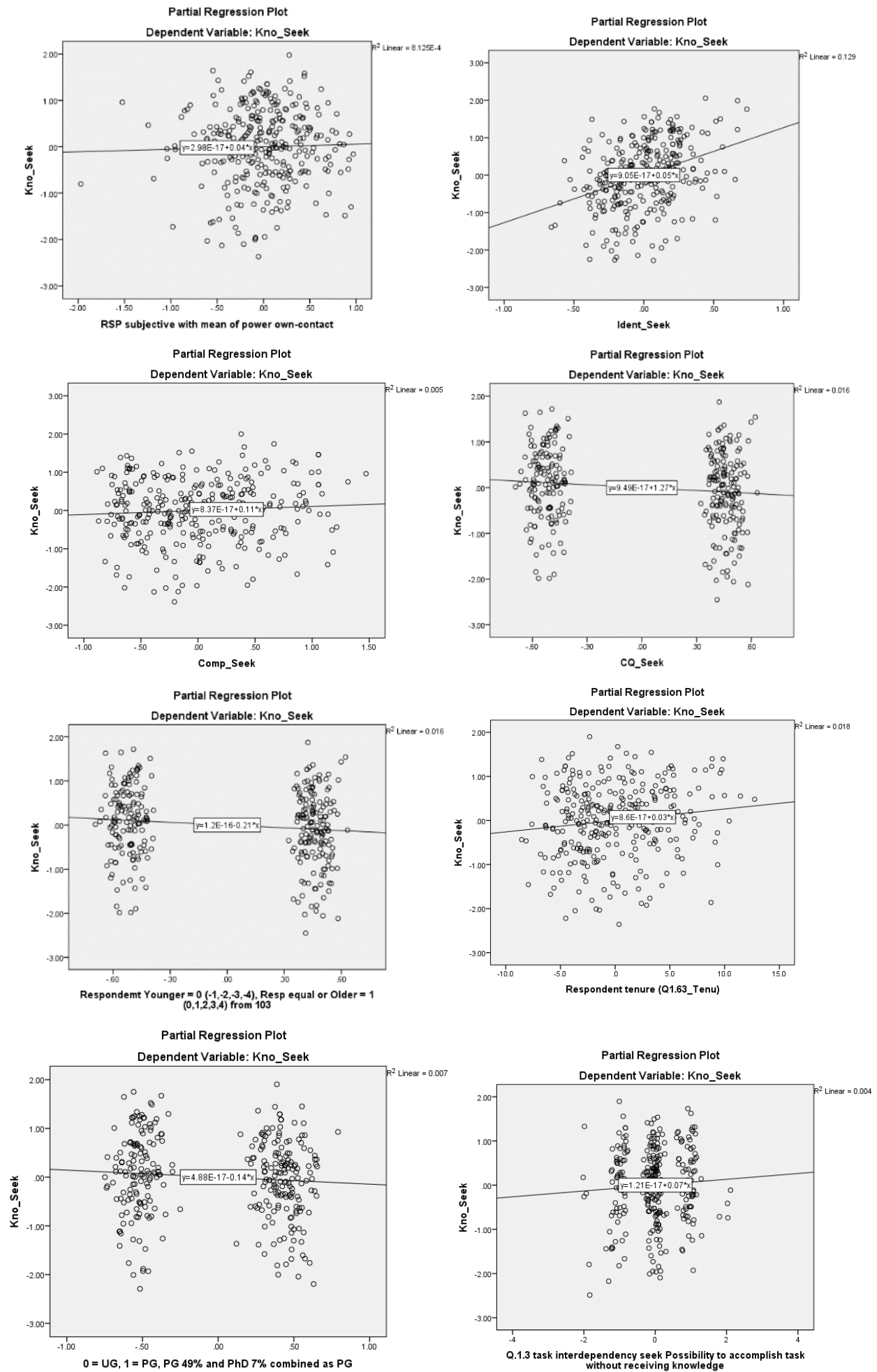
Knowledge sharing with each independent variable
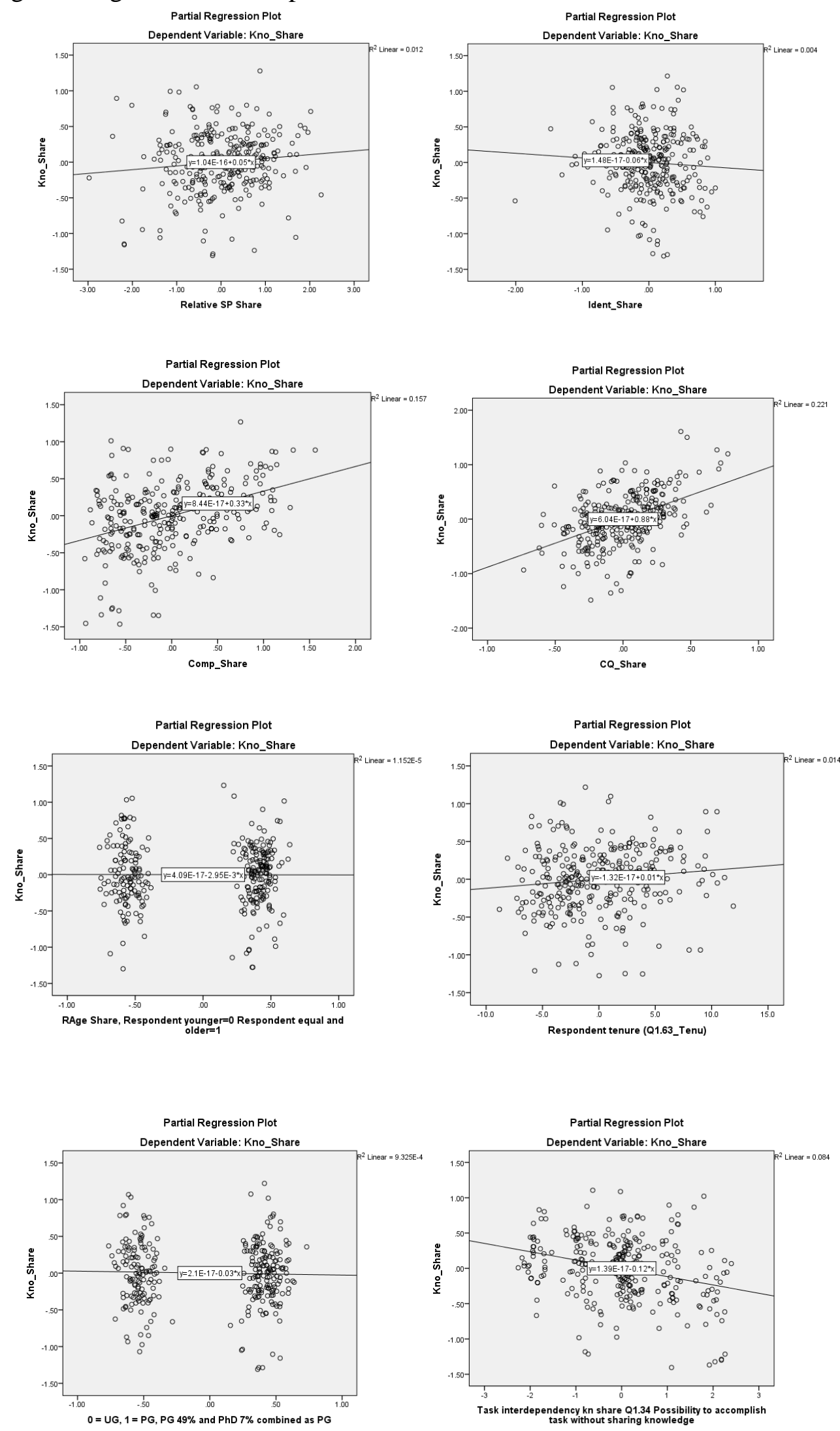


\section{Appendix 6: Test of normality}

Table 1 Kolmogorov-Smirnov ${ }^{\mathrm{a}}$ test for knowledge seeking

\begin{tabular}{|c|c|c|c|}
\hline Variables & Statistic & $\mathrm{df}$ & Sig. \\
\hline KSeeking & .063 & 318 & .004 \\
\hline Sub Power & .151 & 318 & .000 \\
\hline OI & .111 & 318 & .000 \\
\hline Comp & .084 & 318 & .000 \\
\hline CQ & .032 & 318 & $.200^{*}$ \\
\hline Tenure & .120 & 318 & .000 \\
\hline Task & .252 & 318 & .000 \\
\hline
\end{tabular}

*. This is a lower bound of the true significance.

a. Lilliefors Significance Correction

b. The control variables Age and Education were excluded from the test due to them being dichotomous categorical variables

c. Level of significance 0.05

d. Null hypotheses are that the variables are normally distributed

$\mathrm{K}=$ knowledge; $\mathrm{Sub}=$ subsidiary; $\mathrm{OI}=$ Organizational Identification; Comp=Competition; $\mathrm{CQ}=\mathrm{Cultural}$ Intelligence

Table 2 Kolmogorov-Smirnov ${ }^{\mathrm{a}}$ test for knowledge sharing

\begin{tabular}{|c|c|c|c|}
\hline Variables & Statistic & $\mathrm{df}$ & Sig. \\
\hline KSharing & .078 & 297 & .000 \\
\hline Sub Power & .141 & 297 & .000 \\
\hline OI & .106 & 297 & .000 \\
\hline Comp & .072 & 297 & .001 \\
\hline CQ & .039 & 297 & $200^{*}$ \\
\hline Tenure & .124 & 297 & .000 \\
\hline Task & .223 & 297 & .000 \\
\hline
\end{tabular}

*. This is a lower bound of the true significance.

a. Lilliefors Significance Correction

b. The control variables Age and Education were excluded from the test due to them being dichotomous categorical variables

c. Level of significance 0.05

d. Null hypotheses are that the variables are normally distributed

$\mathrm{K}=$ knowledge; $\mathrm{Sub}=$ subsidiary; $\mathrm{OI}=$ Organizational Identification; $\mathrm{Comp}=$ Competition; $\mathrm{CQ}=\mathrm{Cultural}$ Intelligence 


\section{Appendix 7: Homoscedasticity}

Figure 1 Scatterplot for Knowledge Seeking

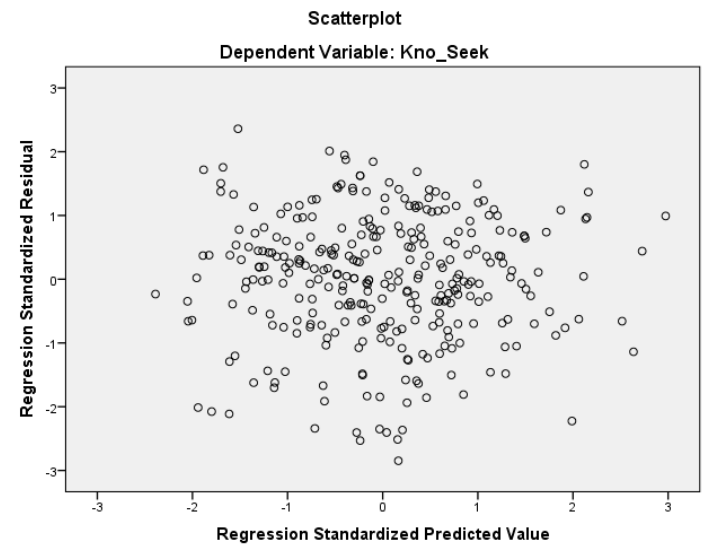

Table 1 Breusch-Pagan and Koenker test statistics and sig-values

\begin{tabular}{lcl}
\hline & LM & Sig \\
\hline BP & 10.961 & .090 \\
Koenker & 11.585 & .072 \\
\hline
\end{tabular}

Null hypothesis: heteroscedasticity not present (homoscedasticity). If sig-value less than 0.05 , reject the null hypothesis.

Figure 2 Scatterplot for Knowledge Sharing

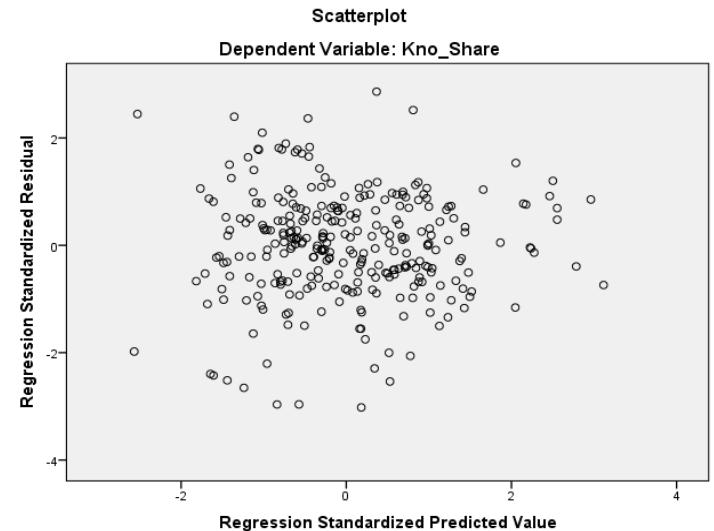

Table 2 Breusch-Pagan and Koenker test statistics and sig-values

\begin{tabular}{lcl}
\hline & LM & Sig \\
\hline BP & 24.080 & .001 \\
Koenker & 18.396 & .005 \\
\hline
\end{tabular}

Null hypothesis: heteroscedasticity not present (homoscedasticity). If sig-value less than 0.05 , reject the null hypothesis. 


\section{Appendix 8 Common method variance}

Table 1 Harman single factor test for knowledge seeking

\begin{tabular}{|c|c|c|c|c|c|c|}
\hline \multicolumn{7}{|c|}{ Total Variance Explained } \\
\hline \multirow{2}{*}{ Factor } & \multicolumn{3}{|c|}{ Initial Eigenvalues } & \multicolumn{3}{|c|}{ Extraction Sums of Squared Loadings } \\
\hline & Total & $\%$ of Variance & Cumulative $\%$ & Total & $\%$ of Variance & Cumulative $\%$ \\
\hline 1 & 1.657 & 18.406 & 18.406 & .916 & 10.174 & 10.174 \\
\hline 2 & 1.324 & 14.706 & 33.112 & & & \\
\hline 3 & 1.153 & 12.808 & 45.920 & & & \\
\hline 4 & 1.117 & 12.408 & 58.327 & & & \\
\hline 5 & .926 & 10.287 & 68.615 & & & \\
\hline 6 & .867 & 9.638 & 78.253 & & & \\
\hline 7 & .780 & 8.670 & 86.923 & & & \\
\hline 8 & .644 & 7.151 & 94.074 & & & \\
\hline 9 & .533 & 5.926 & 100.000 & & & \\
\hline
\end{tabular}

Extraction Method: Principal Axis Factoring.

Table 2 Harman single factor test for knowledge sharing

\begin{tabular}{|c|c|c|c|c|c|c|}
\hline \multicolumn{7}{|c|}{ Total Variance Explained } \\
\hline \multirow{2}{*}{ Factor } & \multicolumn{3}{|c|}{ Initial Eigenvalues } & \multicolumn{3}{|c|}{ Extraction Sums of Squared Loadings } \\
\hline & Total & $\%$ of Variance & Cumulative $\%$ & Total & $\%$ of Variance & Cumulative $\%$ \\
\hline 1 & 1.761 & 19.561 & 19.561 & 1.031 & 11.459 & 11.459 \\
\hline 2 & 1.355 & 15.054 & 34.615 & & & \\
\hline 3 & 1.202 & 13.359 & 47.974 & & & \\
\hline 4 & 1.030 & 11.446 & 59.420 & & & \\
\hline 5 & .905 & 10.053 & 69.473 & & & \\
\hline 6 & .879 & 9.768 & 79.242 & & & \\
\hline 7 & .828 & 9.195 & 88.437 & & & \\
\hline 8 & .627 & 6.965 & 95.402 & & & \\
\hline 9 & .414 & 4.598 & 100.000 & & & \\
\hline
\end{tabular}

Extraction Method: Principal Axis Factoring. 
Table 3 Zero-order correlation table for knowledge seeking

\begin{tabular}{|c|c|c|c|c|c|c|c|}
\hline & & Sub Power & KSeek & OI & Comp & CQ & Task \\
\hline \multirow[t]{3}{*}{ Sub Power } & Pearson Correlation & 1 & & & & & \\
\hline & Sig. (2-tailed) & & & & & & \\
\hline & $\mathrm{N}$ & 333 & & & & & \\
\hline \multirow[t]{3}{*}{ KSeek } & Pearson Correlation & .089 & 1 & & & & \\
\hline & Sig. (2-tailed) & .105 & & & & & \\
\hline & $\mathrm{N}$ & 333 & 333 & & & & \\
\hline \multirow[t]{3}{*}{ OI } & Pearson Correlation & $.129^{*}$ & $.157^{* *}$ & 1 & & & \\
\hline & Sig. (2-tailed) & .018 & .004 & & & & \\
\hline & $\mathrm{N}$ & 333 & 333 & 333 & & & \\
\hline \multirow[t]{3}{*}{ Comp } & Pearson Correlation & $.120^{*}$ & .082 & -.044 & 1 & & \\
\hline & Sig. (2-tailed) & .028 & .135 & .421 & & & \\
\hline & $\mathrm{N}$ & 333 & 333 & 333 & 333 & & \\
\hline \multirow[t]{3}{*}{ CQ } & Pearson Correlation & .084 & $.331^{* *}$ & $.303^{* *}$ & -.078 & 1 & \\
\hline & Sig. (2-tailed) & .124 & .000 & .000 & .156 & & \\
\hline & $\mathrm{N}$ & 333 & 333 & 333 & 333 & 333 & \\
\hline \multirow[t]{3}{*}{ Task } & Pearson Correlation & -.054 & .058 & .055 & .021 & -.082 & 1 \\
\hline & Sig. (2-tailed) & .322 & .289 & .320 & .703 & .137 & \\
\hline & $\mathrm{N}$ & 333 & 333 & 333 & 333 & 333 & 333 \\
\hline
\end{tabular}

*. Correlation is significant at the 0.05 level (2-tailed).

**. Correlation is significant at the 0.01 level (2-tailed).

$\mathrm{K}=$ knowledge; Sub=subsidiary; $\mathrm{OI}=$ Organizational Identification; $\mathrm{Comp}=$ Competition; $\mathrm{CQ}=\mathrm{Cultural}$ Intelligence

Table 4 Partial-correlations table for knowledge seeking

\begin{tabular}{|c|c|c|c|c|c|c|c|c|}
\hline \multicolumn{3}{|c|}{ Control Variables } & Sub Power & KSeek & OI & Comp & CQ & Task \\
\hline \multirow{17}{*}{$\begin{array}{l}\text { Fashion } \\
\text { Consciousness }\end{array}$} & \multirow[t]{2}{*}{ Sub Power } & Correlation & 1.000 & & & & & \\
\hline & & $\begin{array}{l}\text { Significance } \\
\text { (2-tailed) } \\
\text { df }\end{array}$ & $\cdot$ & & & & & \\
\hline & \multirow[t]{3}{*}{ KSeek } & Correlation & .082 & 1.000 & & & & \\
\hline & & $\begin{array}{l}\text { Significance } \\
\text { (2-tailed) }\end{array}$ & .138 & . & & & & \\
\hline & & df & 330 & 0 & & & & \\
\hline & \multirow[t]{3}{*}{ OI } & Correlation & .134 & .167 & 1.000 & & & \\
\hline & & $\begin{array}{l}\text { Significance } \\
\text { (2-tailed) }\end{array}$ & .015 & .002 & . & & & \\
\hline & & df & 330 & 330 & 0 & & & \\
\hline & \multirow[t]{3}{*}{ Comp } & Correlation & .112 & .062 & -.034 & 1.000 & & \\
\hline & & $\begin{array}{l}\text { Significance } \\
\text { (2-tailed) }\end{array}$ & .042 & .263 & .537 & . & & \\
\hline & & df & 330 & 330 & 330 & 0 & & \\
\hline & \multirow[t]{3}{*}{$\overline{C Q}$} & Correlation & .078 & .321 & .313 & -.099 & 1.000 & \\
\hline & & $\begin{array}{l}\text { Significance } \\
\text { (2-tailed) }\end{array}$ & .157 & .000 & .000 & .072 & . & \\
\hline & & df & 330 & 330 & 330 & 330 & 0 & \\
\hline & \multirow[t]{3}{*}{ Task } & Correlation & -.052 & .064 & .052 & .028 & -.077 & 1.000 \\
\hline & & $\begin{array}{l}\text { Significance } \\
\text { (2-tailed) }\end{array}$ & .344 & .242 & .344 & .609 & .159 & $\cdot$ \\
\hline & & & 330 & 330 & 330 & 330 & 330 & 0 \\
\hline
\end{tabular}

$\mathrm{K}=$ knowledge; $\mathrm{Sub}=$ subsidiary; OI=Organizational Identification; Comp=Competition; $\mathrm{CQ}=\mathrm{Cultural}$ Intelligence 
Table 5 Zero-order correlation table for knowledge sharing

\begin{tabular}{|c|c|c|c|c|c|c|c|}
\hline & & Sub Power & KSeek & OI & Comp & $\mathrm{CQ}$ & Task \\
\hline \multirow[t]{2}{*}{ Sub Power } & $\begin{array}{l}\text { Pearson Correlation } \\
\text { Sig. (2-tailed) }\end{array}$ & 1 & & & & & \\
\hline & $\mathrm{N}$ & 333 & & & & & \\
\hline \multirow[t]{3}{*}{ KSeek } & Pearson Correlation & $.176^{* * *}$ & 1 & & & & \\
\hline & Sig. (2-tailed) & .001 & & & & & \\
\hline & $\mathrm{N}$ & 333 & 333 & & & & \\
\hline \multirow[t]{3}{*}{$\mathrm{OI}$} & Pearson Correlation & $.127^{*}$ & $.112^{*}$ & 1 & & & \\
\hline & Sig. (2-tailed) & .020 & .042 & & & & \\
\hline & $\mathrm{N}$ & 333 & 333 & 333 & & & \\
\hline \multirow[t]{3}{*}{ Comp } & Pearson Correlation & .090 & $.335^{* *}$ & -.041 & 1 & & \\
\hline & Sig. (2-tailed) & .101 & .000 & .456 & & & \\
\hline & $\mathrm{N}$ & 333 & 333 & 333 & 333 & & \\
\hline \multirow[t]{3}{*}{ CQ } & Pearson Correlation & $.138^{*}$ & $.405^{* *}$ & $.308^{* *}$ & -.064 & 1 & \\
\hline & Sig. (2-tailed) & .011 & .000 & .000 & .242 & & \\
\hline & $\mathrm{N}$ & 333 & 333 & 333 & 333 & 333 & \\
\hline \multirow[t]{3}{*}{ Task } & Pearson Correlation & .047 & $-.161^{* *}$ & -.062 & .032 & .097 & 1 \\
\hline & Sig. (2-tailed) & .397 & .003 & .260 & .560 & .081 & \\
\hline & $\mathrm{N}$ & 327 & 327 & 327 & 327 & 327 & 327 \\
\hline
\end{tabular}

**. Correlation is significant at the 0.01 level (2-tailed).

*. Correlation is significant at the 0.05 level (2-tailed).

$\mathrm{K}=$ knowledge; $\mathrm{Sub}=$ subsidiary; $\mathrm{OI}=$ Organizational Identification; $\mathrm{Comp}=$ Competition; $\mathrm{CQ}=\mathrm{Cultural}$ Intelligence

Table 6 Partial-correlations table for knowledge sharing

\begin{tabular}{|c|c|c|c|c|c|c|c|c|}
\hline \multicolumn{3}{|c|}{ Control Variables } & \multirow{2}{*}{$\begin{array}{c}\text { Sub Power } \\
1.000\end{array}$} & \multirow[t]{2}{*}{ KSeek } & \multirow[t]{2}{*}{ OI } & \multirow[t]{2}{*}{ Comp } & \multirow[t]{2}{*}{ CQ } & \multirow[t]{2}{*}{ Task } \\
\hline Fashion & Sub Power & Correlation & & & & & & \\
\hline \multirow[t]{17}{*}{ Consciousness } & & $\begin{array}{l}\text { Significance } \\
\text { (2-tailed) }\end{array}$ & . & & & & & \\
\hline & & df & 0 & & & & & \\
\hline & KSeek & Correlation & .164 & 1.000 & & & & \\
\hline & & $\begin{array}{l}\text { Significance } \\
\text { (2-tailed) }\end{array}$ & .003 & . & & & & \\
\hline & & df & 324 & 0 & & & & \\
\hline & OI & Correlation & .114 & .139 & 1.000 & & & \\
\hline & & $\begin{array}{l}\text { Significance } \\
\text { (2-tailed) }\end{array}$ & .039 & .012 & . & & & \\
\hline & & df & 324 & 324 & 0 & & & \\
\hline & Comp & Correlation & .094 & .296 & -.019 & 1.000 & & \\
\hline & & $\begin{array}{l}\text { Significance } \\
\text { (2-tailed) }\end{array}$ & .092 & .000 & .739 & . & & \\
\hline & & df & 324 & 324 & 324 & 0 & & \\
\hline & CQ & Correlation & .138 & .391 & .319 & -.091 & 1.000 & \\
\hline & & $\begin{array}{l}\text { Significance } \\
\text { (2-tailed) }\end{array}$ & .013 & .000 & .000 & .100 & . & \\
\hline & & df & 324 & 324 & 324 & 324 & 0 & \\
\hline & Task & Correlation & .046 & -.172 & -.062 & .031 & .096 & 1.000 \\
\hline & & $\begin{array}{l}\text { Significance } \\
\text { (2-tailed) }\end{array}$ & .404 & .002 & .264 & .575 & .083 & . \\
\hline & & df & 324 & 324 & 324 & 324 & 324 & 0 \\
\hline
\end{tabular}

$\mathrm{K}=$ knowledge; Sub=subsidiary; OI=Organizational Identification; Comp=Competition; $\mathrm{CQ}=\mathrm{Cultural}$ Intelligence 


\section{References}

Adair, W. L., Hideg, I., \& Spence, J. R. (2013). The culturally intelligent team: The impact of team cultural intelligence and cultural heterogeneity on team shared values. Journal of Cross-Cultural Psychology, 44(6), 941-962.

Aggarwal, V. (2016). India top destination for R\&D investments; beats US, China $\mid$ Business Line. Retrieved from http://www.thehindubusinessline.com/info-tech/india-topdestination-for-rd-investments-beats-us-china/article8780331.ece

Allen, T. (1977). Managing the Flow of Technology: Technology Transfer and the Dissemination of Technological Information within the $R \& D$ Organization. Cambridge, Mass: MIT Press.

Allen, T. J., \& Cohen, S. (1969). Information flow in research and development laboratories. Administrative Science Quarterly, 14(1), 12-19.

Alwin, D. F., \& Hauser, R. M. (1975). The decomposition of effects in path analysis. American sociological review, 40(1), 37-47.

Ambos, T. C., \& Ambos, B. (2009). The impact of distance on knowledge transfer effectiveness in multinational corporations. Journal of International Management, 15(1), 1-14.

Ambos, T. C., Ambos, B., \& Schlegelmilch, B. B. (2006). Learning from foreign subsidiaries: An empirical investigation of headquarters' benefits from reverse knowledge transfers. International Business Review, 15(3), 294-312 .

Ambos, T. C., Andersson, U., \& Birkinshaw, J. (2010). What are the consequences of initiative-taking in multinational subsidiaries? Journal of International Business Studies, 41(7), 1099-1118.

Ambos, B., Asakawa, K., \& Ambos, T. C. (2011). A dynamic perspective on subsidiary autonomy. Global Strategy Journal, 1(3-4), 301-316.

Ambrose, D. M., \& Anstey, J. R. (2010). Questionnaire development: Demystifying the process. International Management Review, 6(1), 83.

Ames, R., \& Lau, S. (1982). An attributional analysis of student help-seeking in academic settings. Journal of Educational Psychology, 74(3), 414-423.

Andersson, U., Forsgren, M., \& Holm, U. (2007). Balancing subsidiary influence in the federative MNC: a business network view. Journal of International Business Studies, 38(5), 802-818.

Andersson, U., \& Pahlberg, C. (1997). Subsidiary influence on strategic behaviour in MNCs: an empirical study. International Business Review, 6(3), 319-334.

Ang, S. H. (2014). Research Design for Business \& Management (1st ed.). London: Sage publications.

Ang, S., \& Van Dyne, L. (2008). Handbook of Cultural Intelligence: Theory, Measurement and Application. Armonk, N.Y: M.E. Sharpe.

Ang, S., Van Dyne, L., \& Koh, C. (2006). Personality correlates of the four-factor model of cultural intelligence. Group \& Organization Management, 31(1), 100-123.

Ang, S., Van Dyne, L., Koh, C., Ng, K. Y., Templer, K. J., Tay, C., \& Chandrasekar, N. A. (2007). Cultural intelligence: Its measurement and effects on cultural judgment and decision making, cultural adaptation and task performance. Management and Organization Review, 3(3), 335-371.

Anicich, E. M, Fast, N. J., Halevy, N., \& Galinsky, A.D. (2016). When the bases of social hierarchy collide: Power without status drives interpersonal conflict. Organization Science, 27(1), 123-140.

Appleyard, M. M. (1996). How does knowledge flow? Interfirm patterns in the semiconductor industry. Strategic Management Journal, 17(Winter Special Issue), 137-154.

Argote, L. (1999). Organizational Learning: Creating, Retaining and Transferring 
Knowledge. Boston, MA: Kluwer.

Argote, L., \& Ingram, P. (2000). Knowledge transfer: A basis for competitive advantage in firms. Organizational Behavior and Human Decision Processes, 82(1), 150-169.

Argote, L., Kane, A. A., 2009. Superordinate identity and knowledge creation and transfer in organizations, in: Foss, N., Michailova, S. (Eds.), Knowledge Governance: Processes and Perspectives. Oxford University Press, New York, NY, pp. 166-190.

Argote, L., McEvily, B., Reagans, R., 2003. Managing knowledge in organizations: An integrative framework and review of emerging themes. Management Science, 49(4), 571582.

Armstrong, J. S., \& Overton, T. S. (1977). Estimating nonresponse bias in mail surveys. Journal of Marketing Research, 14(3), 396-402.

Ashforth, B. E., Harrison, S. H., \& Corley, K. G. (2008). Identification in organizations: An examination of four fundamental questions. Journal of Management, 34(3), 325-374.

Ashforth, B. E., \& Mael, F. (1989). Social identity theory and the organization. Academy of Management.The Academy of Management Review, 14(1), 20.

Ashforth, B. E., Schinoff, B. S., \& Rogers, K. M. (2016). "I identify with her , " I identify with him ": Unpacking the dynamics of personal identification in organizations. Academy of Management Review, 41(1), 28-60.

Astley, W. G., \& Sachdeva, P. S. (1984). Structural sources of intraorganizational power: A theoretical synthesis. Academy of Management Review, 9(I), 104-113.

Baker, D. S., Carson, K. D., \& Carson, P. P. (2009). An individual-level examination of the impact of cultural values on organizational identification. Journal of Applied Management and Entrepreneurship, 14(2), 29-43.

Balogun, J., Jarzabkowski, P., \& Vaara, E. (2011). Selling, resistance and reconciliation: A critical discursive approach to subsidiary role evolution in MNEs. Journal of International Business Studies, 42(6), 765-786.

Barner-Rasmussen, W., Ehrnrooth, M., Koveshnikov, A., \& Mäkelä, K. (2014). Cultural and language skills as resources for boundary spanning within the MNC. Journal of International Business Studies, 45(7), 886-905.

Baron, R. M., \& Kenny, D. A. (1986). The moderator-mediator variable distinction in social psychological research: Conceptual, strategic, and statistical considerations. Journal of Personality and Social Psychology, 51(6), 1173.

Bartels, J., Pruyn, A., Jong, M. De, \& Joustra, I. (2007). Multiple organizational identification levels and the impact of perceived external prestige and communication climate. Journal of Organizational Behavior, 17(2), 1-20.

Bartlett, C. A., \& Ghoshal, S. (1989). Managing Across Borders : The Transnational Solution. Boston, MA: Harvard Business School Press.

Bassiri, D. (1988). Large and Small Sample Properties of Maximum Likelihood Estimates for the Hierarchical Linear Model. Unpublished dioctoral dissertation. Michigan State University.

Becker, T. E. (2005). Potential problems in the statistical control of variables in organizational research: A qualitative analysis with recommendations. Organizational Research Methods, 8(3), 274-289.

Becker-Ritterspach, F., \& Dörrenbächer, C. (2011). An organizational politics perspective on intra-firm competition in multinational corporations. Management International Review, 51(4), 533-559.

Berry, T. (2011). The Great Work: Our Way into the Future. New York: Bell Tower.

Birkinshaw, J. (1996). How multinational subsidiary mandates are gained and lost. Journal of International Business Studies, 27(3), 467-495.

Birkinshaw, J., Holm, U., Thilenius, P., \& Arvidsson, N. (2000). Consequences of perception 
gaps in the headquarters-subsidiary relationship. International Business Review, 9(3), 321-344.

Birkinshaw, J., \& Hood, N. (1998). Multinational subsidiary evolution: Capability and charter change in foreign-owned subsidiary companies. Academy of Management Review, 23(4), 773-795.

Birkinshaw, J., \& Lingblad, M. (2005). Intrafirm competition and charter evolution in the multibusiness Firm. Organization Science, 16(6), 674-686.

Bjerregaard, T., \& Klitmoller, A. (2016). Conflictual practice sharing in the MNC: A theory of practice approach. Organization Studies, 37(9), 1271-1295.

Bjorkman, I., Barner-Rasmussen, W., \& Li, L. (2004). Managing knowledge transfer in MNCs: the impact of headquarters control mechanisms. Journal of International Business Studies, 35(5), 443-455.

Blau, P. (1964). Power and exchange in social life. NY: John Wiley \& Sons.

Bliese, P. D. (1998). Group size, ICC values, and group-level correlations: A simulation. Organizational Research Methods, 1(4), 355-373.

Bliese, P. D. (2000). Within-group agreement, non-independence, and reliability: Implications for data aggregation and analysis. In K. J. Klein \& S. W. Kozlowski (Eds.), Multilevel Theory, Research And Methods In Organizations: Foundations, Extensions And New Directions (pp. 349-381). San Francisco: Jossey-Bass.

Bliese, P. D., Chan, D., \& Ployhart, R. E. (2007). Multilevel methods: Future directions in measurement, longitudinal analyses, and nonnormal outcomes. Organizational Research Methods, 10(4), 551-563.

Bogilović, S., Černe, M., \& Škerlavaj, M. (2017). Hiding behind a mask? Cultural intelligence, knowledge hiding, and individual and team creativity. European Journal of Work and Organizational Psychology, 26(5), 710-723.

Bollen, K., \& Lennox, R. (1991). Conventional wisdom on measurement: A structural equation perspective. Psychological Bulletin, 110(2), 305-314.

Bono, J. E., \& McNamara, G. (2011). From the editors. Publishing in AMJ - Part 2: Research design. Academy of Management Journal, 54(4), 657-660.

Borgatti, S. P., \& Cross, R. (2003). A Relational view of information seeking and learning in Social Networks. Management Science, 49(4), 432-445.

Bors, D. (2018). Data analysis for the social sciences: Integrating theory and practice. Sage.

Borsboom, D., Mellenbergh, G. J., \& van Heerden, J. (2004). The concept of validity. Psychological Review, 111(4), 1061-1071.

Bouquet, C., \& Birkinshaw, J. (2008a). Weight versus voice: How foreign subsidiaries gain attention from corporate headquarters. Academy of Management Journal, 51(3), 577-601.

Bouquet, C., \& Birkinshaw, J. (2008b). Managing power in the multinational corporation: How low-power actors gain influence. Journal of Management, 34(3), 477-508.

Bouty, I. (2000). Interpersonal and interaction influences on informal resource exchanges between R\&D. Academy of Management Journal, 43(1), 50-65.

Brewer, J. (2000). Ethnography. Buckingham, U.K: Open University Press.

Brown, T. (2015). Confirmatory factor analysis for applied research (Second edition). New York: The Guilford Press.

Bücker, J. J., Furrer, O., Poutsma, E., \& Buyens, D. (2014). The impact of cultural intelligence on communication effectiveness, job satisfaction and anxiety for Chinese host country managers working for foreign multinationals. The International Journal of Human Resource Management, 25(14), 2068-2087.

Buckley, P. (2011). International integration and coordination in the global factory. Management International Review, 51(2), 269-283.

Buckley, P. J., Clegg, J., \& Tan, H. (2003). The Art of knowledge transfer: Secondary and 
reverse transfer in China's telecommunications manufacturing industry. Management International Review, 43(2), 67-93.

Burnham, K. P., \& Anderson, D. R. (2004). Multimodel inference: understanding AIC and BIC in model selection. Sociological Methods \& Research, 33(2), 261-304.

Byrne, B. M. (2001). Structural equation modeling with AMOS, EQS, and LISREL: Comparative approaches to testing for the factorial validity of a measuring instrument. International Journal of Testing, 1(1), 55-86.

Byrne, B. M. (2010) Structural equation modeling with AMOS: Basic concepts, applications and programming $\left(2^{\text {nd }}\right.$ ed.). New York : Routledge

Cabrera, A., Collins, W. C., \& Salgado, J. F. (2006). Determinants of individual engagement in knowledge sharing. The International Journal of Human Resource Management, 17(2), 245-264.

Cabrera, E. F., \& Cabrera, A. (2005). Fostering knowledge sharing through people management practices. The International Journal of Human Resource Management, 16(5), 720-735.

Caimo, A., \& Lomi, A. (2015). Knowledge Sharing in Organizations: A Bayesian analysis of the role of reciprocity and formal structure. Journal of Management, 41(2), 665-691.

Carmeli, A., Gelbard, R., \& Reiter-Palmon, R. (2013). Leadership, creative problem-solving capacity, and creative performance: The importance of knowledge sharing. Human Resource Management, 52(1), 95-121.

Carter, A. P. (1989). Knowhow trading as economic exchange. Research Policy, 18(3), 155163.

Cerrato, D. (2006). The multinational enterprise as an internal market system. International Business Review, 15(3), 253-277.

Chan, D. (2009). So why ask me? Are self-report data really that bad? In C. E. Lance \& R. J. Vandenberg (Eds.), Statistical and methodological myths and urban legends (pp. 309 336). New York: Routledge.

Chang, S. J., Witteloostuijn, A. Van, \& Eden, L. (2010). From the Editors: Common method variance in international business research. Journal of International Business Studies, 41(2), 178-184.

Chang, Y., Gong, Y., \& Peng, M. W. (2012). Expatriate knowledge transfer, subsidiary absorptive capacity, and subsidiary performance. Academy of Management Journal, 55(4), 927-948.

Chen, C. J., \& Hung, S. W. (2010). To give or to receive? Factors influencing members' knowledge sharing and community promotion in professional virtual communities. Information and Management, 47(4), 226-236.

Chen, M. (1996). Competitor analysis and interfirm rivalry: Toward a theoretical integration. The Academy of Management Review, 21(1), 100-134.

Chen, A. S. Y., Lin, Y. C., \& Sawangpattanakul, A. (2011). The relationship between cultural intelligence and performance with the mediating effect of culture shock: A case from Philippine labourers in Taiwan. International Journal of Intercultural Relations, 35(2), 246-258.

Chen, M. L., \& Lin, C. P. (2013). Assessing the effects of cultural intelligence on team knowledge sharing from a socio-cognitive perspective. Human Resource Management, 52(5), 675-695.

Chhokar, J. S. (2007) India: Diversity and complexity in action. In Chhokar, J. S., Brodbek, \& F. C., House (Eds.), Culture and Leadership across the World the GLOBE Book of Indepth Studies of 25 Societies (pp. 971-1020). Taylor \& Francis Group

Chin, W. W. (1998). The partial least squares approach to structural equation modeling. Modern Methods for Business Research, 295(2), 295-336. 
Chiambaretto, P., Massé, D., \& Mirc, N. (2019). “All for one and one for all?”-knowledge broker roles in managing tensions of internal coopetition: The Ubisoft case. Research Policy, 48(3), 584-600.

Child, J., \& Rodrigues, S. (1996). The role of social identity in the international transfer of knowledge through joint ventures. In S. R. Clegg \& G. Palmer (Eds.), The Politics of Management Knowledge (pp. 46-68). London: Sage.

Chiu, C., Hsu, M., \& Wang, E. (2006). Understanding knowledge sharing in virtual communities: An integration of social capital and social cognitive theories. Decision Support Systems, 42(3), 1872-1888.

Chua, R. (2018). Innovating at cultural crossroads: How multicultural social networks promote idea flow and creativity. Journal of Management, 44(3), 1119-1146.

Chua, R. Y., Morris, M. W., \& Mor, S. (2012). Collaborating across cultures: Cultural metacognition and affect-based trust in creative collaboration. Organizational Behavior and Human Decision Processes, 118(2), 116-131.

Chua, R., \& Ng, K. (2017). Not just how much you know: Interactional effect of cultural knowledge and metacognition on creativity in a global context. Management and Organization Review, 13(2), 281-300.

Ciabuschi, F., Dellestrand, H., \& Kappen, P. (2012). The good, the bad, and the ugly: Technology transfer competence, rent-seeking, and bargaining power. Journal of World Business, 47(4), 664-674.

Ciabuschi, F., Martín Martín, O., \& Ståhl, B. (2010). Headquarters' influence on knowledge transfer performance. Management International Review, 50(4), 471-491.

Cialdini, R. B., Borden, R. J., Thorne, A., Walker, M. R., Freeman, S., \& Sloan, L. R. (1976). Basking in reflected glory: Three (football) field studies. Journal of Personality and Social Psychology, 34(3), 366-375.

Cialdini, R. B., \& Nicholas, M. E. De. (1989). Self-Presentation by association. Journal of Personality and Social Psychology, 57(4), 626-631.

Clogg, C. C., Petkova, E., \& Shihadeh, E. S. (1992). Statistical methods for analyzing collapsibility in regression models. Journal of Educational Statistics, 17(1), 51-74.

Clow, K. E., \& James, K. E. (2014). Questionnaire design. In Essentials of Marketing Research: Putting Research into Practice, (pp. 322-357). USA, Sage Publications, Inc.

Cohen, J. (1988). Statistical power analysis for the behavioral sciences (2nd ed.). Hillsdale, NJ: Erlbaum.

Cohen, W. M., \& Levinthal, D. A. (1990). Absorptive capacity: A new perspective on learning and innovation. Administrative Science Quarterly, 35(1), 128-152.

Coleman, J.S., 1990. Foundations of Social Theory. The Belknap Press of Harvard University Press, Cambridge/London.29

Coltman, T., Devinney, T. M., Midgley, D. F., \& Venaik, S. (2008). Formative versus reflective measurement models: Two applications of formative measurement. Journal of Business Research, 61(12), 1250-1262.

Conroy, K. M., Collings, D. G., \& Clancy, J. (2018). Sowing the seeds of subsidiary influence: Social navigating and political maneuvering of subsidiary actors. Global Strategy Journal, 9 (4), 502-526.

Crespo, C. F., Griffith, D. A., \& Lages, L. F. (2014). The performance effects of vertical and horizontal subsidiary knowledge outflows in multinational corporations. International Business Review, 23(5), 993-1007.

Cross, R., \& Cummings, J. N. (2004). Tie and network correlates of individual performance in knowledge-intensive work. The Academy of Management Journal, 47(6), 928-937.

Crotty, M. (1998). The Foundations of Social Research: Meaning and Perspective in the Research Process. St Leonards, NSW: Allen \& Unwin. 
Crowne, K. A. (2008). What leads to cultural intelligence? Business horizons, 51(5), 391-399.

Crowne, K. A. (2013). Cultural exposure, emotional intelligence, and cultural intelligence: An exploratory study. International Journal of Cross Cultural Management, 13(1), 5-22.

Cui, A. S., Griffith, D. a., Cavusgil, S. T., \& Dabic, M. (2006). The influence of market and cultural environmental factors on technology transfer between foreign MNCs and local subsidiaries: A Croatian illustration. Journal of World Business, 41(2), 100-111.

Dahl, R. A. (1957). The Concept of Power. Behavioral Science, 2(3), 201-215.

Daniels, M. A., \& Greguras, G. J. (2014). Exploring the nature of power distance: Implications for micro-and macro-level theories, processes, and outcomes. Journal of Management, 40(5), 1202-1229.

Darlington, R., \& Hayes, A. (2017). Regression analysis and linear models : concepts, applications, and implementation. New York, New York: The Guilford Press.

Daryanto, A. (2013). Heteroskedasticity-SPSS. [Macro for computing the Breusch-Pagan and Koenker tests in SPSS.] Accessible from https://sites.google.com/site/ahmaddaryanto/ scripts/ Heterogeneity-test.

Dasí, À., Pedersen, T., Gooderham, P.N., Elter, F., Hildrum, J. (2017). The effect of organizational separation on individuals' knowledge sharing in MNCs. Journal of World Business 52(3), 431-446.

Davenport, T. H., \& Prusak, L. (1998). Working Knowledge: How Organisations Manage What They Know. Boston, MA: Harvard Business School Press.

Deloitte. (2011). Research and development expenditure: A concept paper.

Deng, L., \& Gibson, P. (2009). Mapping and modeling the capacities that underlie effective cross-cultural leadership: An interpretive study with practical outcomes. Cross Cultural Management: An International Journal, 16(4), 347-366.

DeVellis, R. (2017). Scale development: theory and applications (Fourth edition). Los Angeles: SAGE.

Dheer, R. J., Lenartowicz, T., \& Peterson, M. F. (2015). Mapping India's regional subcultures: Implications for international management. Journal of International Business Studies, 46(4), 443-467.

Diamantopoulos, A., \& Siguaw, J. A. (2006). Formative versus reflective indicators in organizational measure development: A comparison and empirical illustration. British Journal of Management, 17(4), 263-282.

Dillman, D. A. (2000). Mail and Internet surveys: The tailored design method. New York: John Wiley and Sons, Inc.

Dixon, R. (2007). Electronic vs. conventional surveys. In Handbook of research on electronic surveys and measurements (pp. 104-111). Hershey, PA: IGI Global.

Dixon, G. (2016). Applying the gateway belief model to genetically modified food perceptions: New insights and additional questions. Journal of Communication, 66(6), 888-908.

Doh, J. P., Bunyaratavej, K., \& Hahn, E. D. (2009). Separable but not equal: The location determinants of discrete services offshoring activities. Journal of International Business Studies, 40(6), 926-943.

Dorfman, P., Javidan, M., Hanges, P., Dastmalchian, A., \& House, R. (2012). GLOBE: A twenty year journey into the intriguing world of culture and leadership. Journal of World Business, 47(4), 504-518.

Dörrenbächer, C., \& Geppert, M. (Eds. (2011). Politics and power in the multinational corporation: The role of institutions, interests and identities. Cambridge University Press,.

Dörrenbächer, C., \& Gammelgaard, J. (2010). Multinational corporations, inter-organizational networks and subsidiary charter removals. Journal of World Business, 45(3), 206-216.

Dörrenbächer, C., \& Gammelgaard, J. (2006). Subsidiary role development: The effect of 
micro-political headquarters-subsidiary negotiations on the product, market and valueadded scope of foreign-owned subsidiaries. Journal of International Management, 12(3), 266-283.

Dörrenbächer, C., \& Gammelgaard, J. (2016). Subsidiary initiative taking in multinational corporations: The relationship between power and issue selling. Organization Studies, 37(9), 1249-1270.

Druian, P. R., \& DePaulo, B. M. (1977). Asking a child for help. Social Behavior and Personality, 5(1), 33-39

Duncan, G. T., \& Layard, M. W. J. (1973). A Monte-Carlo study of asymptotically robust tests for correlation coefficients. Biometrika, 60(3), 551-558.

Dutton, J. E., Dukerich, J. M., \& Harquail, C. V. (1994). Organizational images and member Identification. Administrative Science Quarterly, 39(2), 239-263.

Earley, P. C. (2002). Redefining interactions across cultures and organizations: Moving forward with cultural intelligence. Research in Organizational Behavior, 24, 271-299.

Earley, P. C. (2006). Leading cultural research in the future: A matter of paradigms and taste. Journal of International Business Studies, 37(6), 922-931.

Earley, P. C., \& Ang, S. (2003). Cultural intelligence: Individual interactions across cultures. Stanford University Press.

Earley, P. C., \& Mosakowski, E. (2004). Cultural intelligence. Harvard Business Review, 82(10), 139-146.

Edmondson, A. C., \& Mcmanus, S. E. (2007). Methodological fit in field reserach. Academy of Management Review, 32(4), 1155-1179.

Edwards, J. R., \& Bagozzi, R. P. (2000). On the nature and direction of relationships between constructs and measures. Psychological Methods, 5(2), 155.

Efron, B., \& Tibshirani, R. J. (1993). An introduction to the bootstrap. New York: Chapman $\&$ Hall.

Elenkov, D. S., \& Manev, I. M. (2009). Senior expatriate leadership's effects on innovation and the role of cultural intelligence. Journal of World Business, 44(4), 357-369.

Emerson, R. M. (1962). Power - dependence relations. American Sociological Review, 27(1), 31-41.

Enderwick, P., \& Buckley, P. (2019). Beyond supply and assembly relations: Collaborative innovation in global factory systems. Journal of Business Research, 103 (2019), 547-556.

Engle, R. L., \& Crowne, K. A. (2014). The impact of international experience on cultural intelligence: An application of contact theory in a structured short-term programme. Human Resource Development International, 17(1), 30-46.

Enticott, G. (2004). Multiple voices of modernization: some methodological implications. Public Administration, 82(3), 743-756.

Fairchild, A. J., MacKinnon, D. P., Taborga, M. P., \& Taylor, A. B. (2009). R 2 effect-size measures for mediation analysis. Behavior Research Methods, 41(2), 486-498.

Feinberg, S. E., \& Gupta, A. K. (2004). Knowledge spillovers and the assignment of R\&D responsibilities to foreign subsidiaries. Strategic Management Journal, 25(8/9), 823-845.

Felin, T., Foss, N.J., 2005. Strategic organization: A field in search of micro-foundations. Strategic Organization 3(4), 441-455.

Felin, T., Foss, N. J., \& Ployhart, R. E. (2015). The microfoundations movement in strategy and organization theory. Academy of Management Annals, 9(1), 575-632.

Felin, T., \& Hesterly, W. S. (2007). The knowledge-based view, nested heterogeneity, and new value creation: Philosophical Considerations on the Locus of Knowledge. Academy of Management Review, 32(1), 195-218.

Feng, H., Morgan, N. A., \& Rego, L. L. (2015). Marketing department power and firm Performance. Journal of Marketing, 79 (September), 1-20. 
Field, Andy P. Discovering Statistics Using SPSS: (and Sex and Drugs and Rock ' $n$ ' Roll) / Andy Field. 3rd ed. Los Angeles [i.e. Thousand Oaks, Calif.]; London: SAGE Publications, 2009. Print. ISM (London, England).

Fiol, C. M., O'connor, E. J., \& Aguinis, H. (2001). All for one and one for all? The development and transfer of power across organization levels. Academy of Management Review, 26(2), 224-242.

Fornell, C., \& Larcker, D. F. (1981). Structural equation models with unobservable variables and measurement error: Algebra and statistics. JMR, Journal of Marketing Research $18(3), 382-388$.

Foss, N. J. (2007). The emerging knowledge governance approach: Challenges and characteristics. Organization, 14(1), 29-52.

Foss, N. J., Minbaeva, D. B., Pedersen, T., Reinholt, M., 2009. Encouraging knowledge sharing among employees: How job design matters. Human Resource Management 48(6), 871893.

Foss, N. J., Husted, K., \& Michailova, S. (2010). Governing knowledge sharing in organizations: Levels of analysis, governance mechanisms, and research directions. Journal of Management Studies, 47(3), 455-482.

Foss, N. J., \& Pedersen, T. (2002). Transferring knowledge in MNCs: The role of sources of subsidiary knowledge and organizational context. Journal of International Management, 8(1), 49-67.

Foss, N. J., \& Pedersen, T. (2004). Organizing knowledge processes multinational corporation : An Introduction. Journal of International Business Studies, 35(5), 340-349.

Foss, N., Pedersen, T., Reinholt Fosgaard, M., \& Stea, D. (2015). Why Complementary HRM Practices Impact Performance: The Case of Rewards, Job Design, and Work Climate in a Knowledge-Sharing Context. Human Resource Management, 54(6), 955-976.

Fragale, A., Overbeck, J., \& Neale, M. (2011). Resources versus respect: Social judgments based on targets' power and status positions. Journal of Experimental Social Psychology, 47(4), 767-775.

French, J. R. P., \& Raven, B. (1968). The bases of social power. In D. Cartwright \& A. Zander (Eds.), Group Dynamics (pp. 259-269). New York: Harper and Row.

French, J. R. P., \& Raven, B. (1959). The bases of social power. In D. Cartwright (Ed.), Studies in Social Power (pp. 529-69). Ann Arbor, MI: University of Michigan Press.

Frenkel, M. (2008). The multinational corporation as a third space: Rethinking international management discourse on knowledge transfer through homi bhabha. Academy of Management Review, 33(4), 924-942.

Friedkin, N. E., \& Simpson, M. J. (1985). Effects of competition on members' identification with their subunits. Administrative Science Quarterly, 30(3), 377-394.

Fritz, M. S., \& MacKinnon, D. P. (2007). Required sample size to detect the mediated effect. Psychological Science, 18(3), 233-239.

Frost, T. S., \& Zhou, C. (2005). R\&D Co-practice and "reverse" knowledge integration in multinational Firms. Journal of International Business Studies, 3(2), 128-128.

Furuya, N., Stevens, M. J., Bird, A., Oddou, G., \& Mendenhall, M. (2009). Managing the learning and transfer of global management competence: Antecedents and outcomes of Japanese repatriation effectiveness. Journal of International Business Studies, 40(2), 200215.

Gagné, M., 2009. A model of knowledge-sharing motivation. Human Resource Management $48,571-589.30$

Gagné, M., \& Deci, E. L. (2005). Self-determination theory and work motivation. Journal of Organizational Behavior, 26(4), 331-362.

Galang, M. C., \& Ferris, G. R. (1997). Human resource department power and influence 
through symbolic action. Human Relations, 50(11), 1403-1426.

Galunic, D. C., \& Eisenhardt, K. M. (1996). The evolution of intracorporate domains : divisional charter losses in high-technology, multidivisional corporations. Organization Science, 7(3), 255-282.

Gammelgaard, J. (2009). Issue selling and bargaining power in intrafirm competition: The differentiating impact of the subsidiary management composition. Competition \& Change, 13(3), 214-228.

Gaskin, J., (2016), "Excel Stats Tools", Stats Tools Package. http://statwiki.kolobkreations.com

Geppert, M., Becker-Ritterspach, F., \& Mudambi, R. (2016). Politics and power in multinational companies: Integrating the international business and organization studies perspectives. Organization Studies, 37(9), 1209-1225.

Geppert, M., \& Dörrenbächer, C. (2014). Politics and power within multinational corporations: Mainstream studies, emerging critical approaches and suggestions for future research. International Journal of Management Reviews, 16(2), 226-244.

Ghoshal, S., \& Bartlett, C. a. (1990). The Multinational corporation as an interorganizational network. Academy of Management Review, 15(4), 603-625.

Ghoshal, S., \& Nohria, N. (1989). Internal differentiation within multinational corporations. Strategic Management Journal, 10(4), 323-337.

Gooderham, P., Minbaeva, D. B., \& Pedersen, T. (2011). Governance mechanisms for the promotion of social capital for knowledge transfer in multinational corporations. Journal of Management Studies, 48(1), 123-150.

Gouldner, A. W. (1960). The norm of reciprocity: A preliminary statement. American Sociological Review, 25(2), 161-178.

Govindarajan, V., \& Fisher, J. (1990). Strategy, control systems, and resource sharing: Effects on business-unit performance. Academy of Management journal, 33(2), 259-285.

Grandori, A., 2001. Neither hierarchy nor identity: Knowledge -governance mechanisms and the theory of the firm. Journal of Management and Governance 5, 381-399.

Grant, R. M. (1996). Toward a knowledge-based theory of the firm. Strategic Management Journal, 17(Winter 1996), 109-122.

Grant, R. M., \& Baden-Fuller, C. (1995). A knowledge-based theory of inter-firm collaboration. Academy of Management Journal, 17-21.

Green, S. B. (1991). How many subjects does it take to do a regression analysis? Multivariate Behavioral Research, 26, 499-510.

Gregersen, H., \& Black, J. S. (1992). Antecedents to commitment to a parent company and a foreign operation. Academy of Management Journal, 35(1), 65-90.

Grosse, R. (1996). International technology transfer in services. Journal of International Business Studies, 27(4), 781-800.

Groves, K. S., \& Feyerherm, A. E. (2011). Leader cultural intelligence in context: Testing the moderating effects of team cultural diversity on leader and team performance. Group \& Organization Management, 36(5), 535-566.

Groves, K. S., Feyerherm, A., \& Gu, M. (2015). Examining cultural intelligence and crosscultural negotiation effectiveness. Journal of Management Education, 39(2), 209-243.

Guba, E. G., \& Lincoln, Y. S. (1994). Competing paradigms in qualitative research. In: Denzin N, Lincoln YS, eds. Handbook of qualitative research. Thousand Oaks, CA: Sage Handbook of qualitative research, 2(163-194), 105.

Guba, E. G., \& Lincoln, Y. S. (2005). Paradigmatic Controversies, Contradictions, and Emerging Confluences. In N. K. Denzin \& Y. S. Lincoln (Eds.), The Sage handbook of qualitative research (pp. 191-215). Thousand Oaks, CA: Sage Publications Ltd.

Gupta, A. K., \& Govindarajan, V. (1984). Business unit strategy, managerial characteristics, 
and business unit effectiveness at strategy implementation. Academy of Management Journal, 27(1), 25-41.

Gupta, A. K., \& Govindarajan, V. (1991). Knowledge flows and the structure of control within multinational corporations. The Academy of Management Review, 16(4), 768-792.

Gupta, A. K., \& Govindarajan, V. (2000). Knowledge flows within multinational Corporations. Strategic Management Journal, 21(4), 473-496.

Gupta, R., \& Gupta, B. M. (2014). Foreign MNC R\&D centers in India: A study of their publications, 2003-12. DESIDOC Journal of Library and Information Technology, 34(4), 287-292.

Haas, M. R., \& Cummings, J. N. (2015). Barriers to knowledge seeking within MNC teams: Which differences matter most? Journal of International Business Studies, 46(1), 36-62.

Hair, J. F., Anderson, R. E., Tatham, R. L., \& Black, W. C. (1995). Multivariate Data Analysis with Readings, 3d ed. Englewood Cliffs, NJ: Prentice Hall, 1995

Hair, J. F., Celsi, M., Money, A., Samouel, P., \& Page, M. (2016). The Essentials of Business Research Methods: Third Edition. Taylor and Francis.

Hair, J. F., Black, W. C., \& Babin, B. J., \& Anderson. (2010). Multivariate Data Analysis. Upper Saddle River, NJ: Prentice Hall.

Hakanson, L., \& Nobel, R. (2001). Organizational characteristics and reverse technology transfer. Management International Review, 41(4), 395-420.

Hansen, M. T. (1999). The search-transfer problem The role of weak ties in sharing knowledge across organization subunits. Administrative Science Quarterly, 44(1), 82-111.

Hansen, M. T. (2002). Knowledge Networks: Explaining effective knowledge sharing in multiunit companies. Organization Science, 13(3), 232-248.

Hansen, M. T., \& Lovas, B. (2004). How do multinational companies leverage technological competencies? Moving from single to interdependent explanations. Strategic Management Journal, 25(8-9), 801-822.

Hansen, M. T., Mors, M. L., \& Lovas, B. (2005). Knowledge sharing in oranizations: Multiple networks, multiple phases. Academy of Management Journal, 48(5), 776-793.

Hariri, A., \& Roberts, P. (2015). Adoption of innovation within universities: Proposing and testing an initial model. Creative Education, 6(02), 186.

Harman, H. H. (1967), Modern Factor Analysis, University of Chicago Press, Chicago, IL.

Harrison, N. (2012). Investigating the impact of personality and early life experiences on intercultural interaction in internationalised universities. International Journal of Intercultural Relations, 36(2), 224-237.

Hayes, A. F. (2012). PROCESS: A versatile computational tool for observed variable mediation, moderation, and conditional process modeling [White paper].

Hayes, A. F., 2013. Introduction to Mediation, Moderation, and Conditional Process Analysis: A Regression-Based Approach. The Guilford Press, New York.

Hayes, A. F. (2015). An index and test of linear moderated mediation. Multivariate Behavioral Research, 50(1), 1-22.

Hayes, A. F. (1996). Permustat. Behavior Research Methods, Instruments, \& Computers, 28(3), 473-475.

Hayes, A., \& ProQuest. (2018a). Introduction to Mediation, Moderation, and Conditional Process Analysis: A Regression-based Approach (Second edition). New York: The Guilford Press.

Hayes, A. F. (2018b). Partial, conditional, and moderated moderated mediation: Quantification, inference, and interpretation. Communication Monographs, 85(1), 4-40.

Hayes, A. F., \& Cai, L. (2007). Using heteroskedasticity-consistent standard error estimators in OLS regression: An introduction and software implementation. Behavior Research Methods, 39(4), 709-722. 
Hayes, A. F., Montoya, A. K., \& Rockwood, N. J. (2017). The analysis of mechanisms and their contingencies: PROCESS versus structural equation modeling. Australasian Marketing Journal (AMJ), 25(1), 76-81.

Hayes, A. F., \& Rockwood, N. J. (2017). Regression-based statistical mediation and moderation analysis in clinical research: Observations, recommendations, and implementation. Behaviour Research and Therapy, 98(2017), 39-57.

Hayes, A. F., \& Scharkow, M. (2013). The relative trustworthiness of inferential tests of the indirect effect in statistical mediation analysis: Does method really matter? Psychological Science, 24(10), 1918-1927.

Hedlund, G. (1986). The hypermodern MNC - a heterarchy? Human Resource Management, 25(1), 9-35.

He, W., Fang, Y., \& Wei, K. W. (2009). The role of trust in promoting organizational knowledge seeking using knowledge management systems: An empirical investigation. Journal of the American Society For Information Science and Technology, 60(3), 526537.

Heiman, B., \& Nickerson, J. (2002). Towards reconciling transaction cost economics and the knowledge-based view of the firm: The Context of interfirm collaborations. International Journal of the Economics of Business, 9(1), 97-116.

Hickson, D. J., Hinigs, C. R., Lee, C. A., Schneck, R. E., \& Pennings, J. M. (1971). A strategic contingencies' theory of intra-organizational power. Administrative Science Quarterly, 16(2), 216-229.

Hippel, E. von. (1987). Cooperation between rivals: Informal know-how trading. Research Policy, 16(6), 291-302.

Hippel, E. Von. (1988). The Sources of Innovation. Cambridge: MIT press.

Hofmann, D. A. (1997). An overview of the logic and rationale of hierarchical linear models. Journal of Management, 23(6), 723-744.

Hofstede, G. (1980). Motivation, leadership, and organization: do American theories apply abroad? Organizational Dynamics, 9(1), 42-63.

Hofstede, G. B. (1991). Cultures and organizations-software of the mind. UK: McGrawHill Companies Inc.

Hofstede, G. (2001). Culture's Consequences: Comparing Values, Behaviors, Institutions and Organizations Across Nations (2nd ed.). United States of America: Sage publications.

Holtbrügge, D., \& Berg, N. (2004). Knowledge transfer in multi- national corporations: Evidence from German firms. Management International Review, 3(Special Issue), 129145.

Hon, A. H., \& Lu, L. (2015). Are we paid to be creative? The effect of compensation gap on creativity in an expatriate context. Journal of World Business, 50(1), 159-167.

Hornsey, M. J., Spears, R., Cremers, I., \& Hogg, M. a. (2003). Relations between high and low power groups: the importance of legitimacy. Personality and Social Psychology Bulletin, 29(2), 216-227.

House, R. J., Dorfman, P. W., Javidan, M., Hanges, P. J., \& de Luque, M. F. S. (2013). Strategic leadership across cultures: GLOBE study of CEO leadership behavior and effectiveness in 24 countries. Sage Publications.

House, R., \& Global leadership organizational behavior effectiveness research program. (2004). Culture, leadership, and organizations: The GLOBE study of 62 societies / edited by Robert J. House ... [et al.]. Thousand Oaks, CA: Sage Publications.

House, R., Javidan, M., Hanges, P., \& Dorfman, P. (2002). Understanding cultures and implicit leadership theories across the globe: an introduction to project GLOBE. Journal of World Business, 37(1), 3-10.

Huff, K. C., Song, P., \& Gresch, E. B. (2014). Cultural intelligence, personality, and cross- 
cultural adjustment: A study of expatriates in Japan. International Journal of Intercultural Relations, 38, 151-157.

Husted, K., \& Michailova, S. (2002). Knowledge sharing in Russian companies with Western participation. Management International, 6(2), 17-28.

Husted, K., Michailova, S., Minbaeva, D.B., Pedersen, T., 2012. Knowledge-sharing hostility and governance mechanisms: an empirical test. Journal of Knowledge Management 16(5), 754-773.

Hutchings, K., \& Michailova, S. (2004). Facilitating knowledge sharing in Russian and Chinese subsidiaries: the role of personal networks and group membership. Journal of Knowledge Management, 8(2), 84-94.

Hwang, A., \& Francesco, A. M. (2010). The influence of individualism-collectivism and power distance on use of feedback channels and consequences for learning. Academy of Management Learning \& Education, 9(2), 243-257.

Hwang, A., Francesco, A. M., \& Kessler, E. (2003). The relationship between individualismcollectivism, face, and feedback and learning processes in Hong Kong, Singapore, and the United States. Journal of Cross-Cultural Psychology, 34(1), 72-91.

Imai, L., \& Gelfand, M. J. (2010). The culturally intelligent negotiator: The impact of cultural intelligence (CQ) on negotiation sequences and outcomes. Organizational Behavior and Human Decision Processes, 112(2), 83-98.

Ingram, P., \& Yue, L. Q. (2008). Structure, affect and identity as bases of organizational competition and cooperation. The Academy of Management Annals, 2(1), 275-303.

Inkpen, A. C., \& Tsang, E. W. K. (2005). Social capital, networks, and knowledge transfer. Academy of Management Review, 30(1), 146-165.

Jankowicz, A. D. (2000). Business research methods. London: Thomson Learning.

Jarvis, C. B., MacKenzie, S. B., \& Podsakoff, P. M. (2003). A critical review of construct indicators and measurement model misspecification in marketing and consumer research. Journal of Consumer Research, 30(2), 199-218.

Javidan, M. (2004) 'Performance orientation as a cultural dimension', In: R.J. House, P.J. Hanges, M. Javidan, P.W. Dorfman and V. Gupta (eds.) Culture, Leadership, and Organizations: The GLOBE Study of 62 Societies, (pp: 239-276). Thousand Oaks, CA: Sage.

Jonker, J., \& Pennink, B. (2010). The essence of research methodology: A concise guide for master and PhD students in management science. Verlag Berlin Heidelberg: Springer.

Kachra, A., \& White, R. E. (2008). Know-how transfer: The role of social , economic / competitive , and firm boundary factors. Strategic Management Journal, 29(4), 425-445.

Karabenick, S. A., \& Knapp, J. R. (1988). Help seeking and the need for academic assistance. Journal of Educational Psychology, 80(3), 406-408.

Kaše, R., Paauwe, J., \& Zupan, N. (2009). HR Practices, international relations, and intrafirm knowledge transfer In knowledge-intensive firms: A social network perspective. Human Resource Management, 48(4), 615-639.

Katz, R., \& Allen, T. (1982). Investigating the not invented here (NIH) syndrome: A look at the performance, tenure, and communication patterns of $50 \mathrm{R} \& \mathrm{D}$ Project Groups. $R \& D$ Management, 12(1), 7-20.

Kehoe, R. R., \& Wright, P. M. (2013). The impact of high-performance human resource practices on employees' attitudes and behaviors. Journal of Management, 39(2), 366-391.

Keller, R. T., \& Holland, W. E. (1983). Communicators and innovators in research and development organizations. Academy of Management Journal, 26(4), 742-749.

Keltner, D., Gruenfeld, D. H., \& Anderson, C. (2003). Power, approach, and inhibition. Psychological Review, 110(2), 265-284.

Kilduff, G., \& Galinsky, A. (2013). From the ephemeral to the enduring: How approach- 
oriented mindsets lead to greater status. Journal of Personality and Social Psychology, 105(5), 816-831.

Kilduff, D., \& Krackhardt, M. (1994). Bringing the Individual Back in : A structural analysis of the internal market for reputation in organizations. Academy of Management Journal, 37(1), 87-108.

Kim, Y. J., \& Van Dyne, L. (2012). Cultural intelligence and international leadership potential: The importance of contact for members of the majority. Applied Psychology, 61(2), 272294.

Klitmøller, A., \& Lauring, J. (2013). When global virtual teams share knowledge: Media richness, cultural difference and language commonality. Journal of World Business, 48(3), 398-406.

Kogut, B., \& Zander, U. (1992). Knowledge of the firm , combinative capabilities , and the replication of technology. Organization Science, 3(3), 383-397.

Kogut, B., \& Zander, U. (1993). Knowledge of the firm and the evolutionary theory of the multinational corporation. Journal of International Business Studies, 24(4), 625-645.

Kogut, B., \& Zander, U. (2003). Knowledge of the firm and the evolutionary theory of the multinational corporation. Journal of International Business Studies, 34(6), 516-529.

Koo Moon, H., Kwon Choi, B., \& Shik Jung, J. (2012). Previous international experience, cross-cultural training, and expatriates' cross-cultural adjustment: Effects of cultural intelligence and goal orientation. Human Resource Development Quarterly, 23(3), 285330.

Kostova, T. (1999). Transnational transfer of strategic organizational practices: A contextual perspective. Academy of Management Review 24(2), 308-324.

Kostova, T., \& Roth, K. (2002). Adoption of an organizational practice by subsidiaries of multinational corporations: Institutional and relational effects. Academy of Management Journal 45(1), 215-233.

Kotabe, M., Dunlap-Hinkler, D., Parente, R., \& Mishra, H. a. (2007). Determinants of crossnational knowledge transfer and its effect on firm innovation. Journal of International Business Studies, 38(2), 259-282.

Kreiner, K., \& Schultz, M. (1993). Informal collaboration in R\&d. The formation of networks across organizations. Organization Studies, 14(2), 189-209.

Krishnan, R., Martin, X., \& Noorderhaven, N. G. (2006). When does trust matter to alliance performance?. Academy of Management Journal, 49(5), 894-917.

Krishnan, T. N., \& Poulose, S. (2016). Response rate in industrial surveys conducted in India: Trends and implications. IIMB Management Review, 28(2), 88-97.

Kumar, N., Stern, L. W., \& Anderson, J. C. (1993). Conducting inter-organizational research using key informants. Academy of Management Journal, 36(6), 1633-1651.

Kuvaas, B., Buch, R., \& Dysvik, A. (2012). Perceived training intensity and knowledge sharing: Sharing for intrinsic and prosocial reasons. Human Resource Management, 51(2), 167-187.

Kwak, N., \& Radler, B. (2002). A comparison between mail and web surveys: Response pattern, respondent profile, and data quality. Journal of Official Statistics, 18(2), 257.

Lai, H.-M., Chen, C.-P., \& Chang, Y.-F. (2014). Determinants of knowledge seeking in professional virtual communities. Behaviour \& Information Technology, 33(5), 522-535.

Lam, A. (2003). Organizational learning in multinationals: R\&D networks of Japanese and US MNEs in the UK. Journal of Management Studies, 40(3), 673-703.

Lazarova, M., \& Tarique, I. (2005). Knowledge transfer upon repatriation. Journal of World Business, 40(4), 361-373.

Leary, M. R., \& Kowalski, R. M. (1990). Impression management : A literature review and two-component model. Psychological Bulletin, 107(I), 34-47. 
Lee, F. (1997). When the going gets tough, do the tough ask for help? Help seeking and power motivation in organizations. Organizational Behavior and Human Decision Processes, 72(3), 336-63.

Le, S., \& Kroll, M. (2017). CEO international experience: Effects on strategic change and firm performance. Journal of International Business Studies, 48(5), 573-595.

Lee, L. Y., \& Sukoco, B. M. (2010). The effects of cultural intelligence on expatriate performance: The moderating effects of international experience. The International Journal of Human Resource Management, 21(7), 963-981.

Lee, S., Adair, W. L., \& Seo, S. J. (2013). Cultural perspective taking in cross-cultural negotiation. Group Decision and Negotiation, 22(3), 389-405.

Lertwannawit, A., \& Mandhachitara, R. (2012). Interpersonal effects on fashion consciousness and status consumption moderated by materialism in metropolitan men. Journal of Business Research, 65(10), 1408-1416.

Li, M., \& Gao, F. (2003). Why Nonaka highlights tacit knowledge: a critical review. Journal of Knowledge Management, 7(4), 6-14

Lincoln, Y., Guba, E. (2002). The only generalization is: There is no generalization. In Gomm, R., Hammersley, M., Foster, P. (Eds.), Case Study Method (pp. 27-44). London: Sage.

Liening, S. H., Mehta, P. H., \& Josephs, R. A. (2012). Competition. Encyclopedia of Human Behavior, USA: Elseiver, Inc.

Lietz, P. (2010). Research into questionnaire design. International Journal of Market Research, 52(2), 249-272.

Lin, Y. C., Chen, A. S. Y., \& Song, Y. C. (2012). Does your intelligence help to survive in a foreign jungle? The effects of cultural intelligence and emotional intelligence on crosscultural adjustment. International Journal of Intercultural Relations, 36(4), 541-552.

Lindell, M. K., \& Whitney, D. J. (2001). Accounting for common method variance in crosssectional research designs. Journal of Applied Psychology, 86(1), 114.

Ling, Y., Floyd, S. W., \& Baldridge, D. C. (2005). Toward a model of issue-selling by subsidiary managers in multinational organizations. Journal of International Business Studies, 36(6), 637-654.

Liyanage, C., Elhag, T., Ballal, T., \& Li, Q. (2009). Knowledge communication and translation-a knowledge transfer model. Journal of Knowledge management, 13(3), 118131.

Llopis, O., \& Foss, N. J. (2016). Understanding the climate-knowledge sharing relation: The moderating roles of intrinsic motivation and job autonomy. European Management Journal, 34(2), 135-144.

Lorenz, M. P., Ramsey, J. R., \& Richey Jr, R. G. (2018). Expatriates' international opportunity recognition and innovativeness: The role of metacognitive and cognitive cultural intelligence. Journal of World Business, 53(2), 222-236.

Luo, Y. (2003). Market-seeking MNEs in an emerging market: How parent-subsidiary links shape overseas success. Journal of International Business Studies, 34(3), 290-309.

Luo, Y. (2005). Toward coopetition within a multinational enterprise: a perspective from foreign subsidiaries. Journal of World Business, 40(1), 71-90.

Maanen, J. Van, SØrensen, J. B., \& Mitchell, T. R. (2007). The interplay between theory and method. Academy of Management Review, 32(4), 1145-1154.

MacKinnon, D. P., Lockwood, C. M., Hoffman, J. M., West, S. G., \& Sheets, V. (2002). A comparison of methods to test mediation and other intervening variable effects. Psychological Methods, 7(1), 83.

MacKinnon, D. P., Warsi, G., \& Dwyer, J. H. (1995). A simulation study of mediated effect measures. Multivariate Behavioral Research, 30(1), 41-62.

MacNab, B., Brislin, R., \& Worthley, R. (2012). Experiential cultural intelligence 
development: Context and individual attributes. The International Journal of Human Resource Management, 23(7), 1320-1341.

MacNab, B. R., \& Worthley, R. (2012). Individual characteristics as predictors of cultural intelligence development: The relevance of self-efficacy. International Journal of Intercultural Relations, 36(1), 62-71.

McCrae, R. R., \& Costa, P. T. (1989). The structure of interpersonal traits: Wiggins's circumplex and the five-factor model. Journal of Personality and Social Psychology, 56(4), 586.

Mael, F., \& Ashforth, B. E. (1992). Alumni and their alma mater: A partial test of the reformulated model of organizational identification. Journal of Organizational Behavior, $13(2), 103-123$.

Magee, J. C., \& Galinsky, A. D. (2008). Social hierarchy: The self reinforcing nature of power and status. The Academy of Management Annals, 2(1), 351-398.

Mahnke, V., Pedersen, T., \& Venzin, M. (2005). The impact of knowledge management on MNC subsidiary performance: The Role of Absorptive Capacity. Management International Review, 45(2), 101-119.

Mäkelä, K., Andersson, U., \& Seppälä, T. (2012). Interpersonal similarity and knowledge sharing within multinational organizations. International Business Review, 21(3), 439451.

Mäkelä, K., \& Brewster, C. (2009). Interunit interaction contexts, interpersonal social capital, and the differing levels of knowledge sharing. Human Resource Management, 48(4), 591613.

Mäkelä, K., Kalla, H. K., \& Piekkari, R. (2007). Interpersonal similarity as a driver of knowledge sharing within multinational corporations. International Business Review, 16(2007), 1-22.

Malek, M. A., \& Budhwar, P. (2013). Cultural intelligence as a predictor of expatriate adjustment and performance in Malaysia. Journal of World Business, 48(2), 222-231.

Malhotra, N. K., Kim, S. S., \& Patil, A. (2006). Common method variance in IS research: A comparison of alternative approaches and a reanalysis of past research. Management Science, 52(12), 1865-1883.

March, J. G. (1962). The business firm as a political coalition. The Journal of Politics, 24(4), 662-678.

Marsh, H.W., Wen, Z., Hau, K., \& Nagengast, B. (2013). Structural equation models of latent interactions and quadratic effects. In: Hancock, G.R., Mueller, R.O. (Eds.), A Second Course in Structural Equation Modeling, second ed. Information Age, Greenwich, CT. M

Mathur, P., Aycan, Z., \& Kanungo, R. N. (1996). Work cultures in Indian organisations: A comparison between public and private sector. Psychology and Developing Societies, 8(2), 199-222.

Matzler, K., Renzl, B., Müller, J., Herting, S., \& Mooradian, T.A. (2008). Personality traits and knowledge sharing. Journal of Economic Psychology 29, 301-313.

Mechanic, D. (1962). Sources of power of lower participants in complex organizations. Administrative Science Quarterly, 7(3), 349-364.

Medcof, J. W. (2001). Resource-based strategy and managerial power in networks of internationally dispersed technology units. Strategic Management Journal, 22(11), 9991012.

Mertens, D. M. (2004, August). Transformative research and evaluation and dimensions of diversity. In Proceedings of the Sixth International Conference on Social Science Methodology: RC 33 Logic \& Methodology (pp. 17-20).

Michailova, S., \& Husted, K. (2003). Knowledge-sharing hostility in Russian firms. California Management Review, 45(3), 59-77. 
Michailova, S., \& Hutchings, K. (2006). National cultural influences on knowledge sharing: A comparison of China and Russia. Journal of Management Studies 43(3), 383-405.

Michailova, S., \& Minbaeva, D.B. (2012). Organizational values and knowledge sharing in multinational corporations: The Danisco case. International Business Review 21(1), 5970.

Michailova, S., \& Mustaffa, Z. (2012). Subsidiary knowledge flows in multinational corporations: Research accomplishments, gaps, and opportunities. Journal of World Business, 47(3), 383-396.

Minbaeva, D. B. (2007). Knowledge transfer in multinational corporations. Management International Review, 47(4), 567-593.

Minbaeva, D. B. (2008). HRM practices affecting extrinsic and intrinsic motivation of knowledge receivers and their effect on intra-MNC knowledge transfer. International Business Review, 17(6), 703-713.

Minbaeva, D. B. (2013). Strategic HRM in building micro-foundations of organizational knowledge based performance. Human Resource Management Review 23(4), 378-390.

Minbaeva, D. B., Mäkelä, K., \& Rabbiosi, L. (2012). Linking HRM and knowledge transfer via individual-level mechanisms. Human Resource Management, 51(3), 387-405.

Minbaeva, D. B., Pedersen, T., Björkman, I., Fey, C. F., \& Park, H. J. (2003). MNC knowledge transfer, subsidiary absorptive capacity, and HRM. Journal of International Business Studies, 34(6), 586-599.

Mor, S., Morris, M. W., \& Joh, J. (2013). Identifying and training adaptive cross-cultural management skills: The crucial role of cultural metacognition. Academy of Management Learning \& Education, 12(3), 453-475.

Morris, S. S., Zhong, B., \& Makhija, M. (2015). Going the distance: The pros and cons of expanding employees' global knowledge reach. Journal of International Business Studies, 46(5), 552-573.

Morrison, EW, Chen, YR, \& Salgado, SR (2004). Cultural differences in newcomer feedback: A comparison of the United States and Hong Kong. Applied Psychology, 53(1), 1-22.

Motohashi, K. (2015). Global Business Strategy-Multinational Corporations Venturing into Emerging Markets. Springer Tokyo Heidelberg New York Dordrecht London.

Mrinalini, N., Nath, P., \& Sandhya, G. D. (2013). Foreign direct investment in R \& D in India. Current Science, 105(6), 767-773.

Mudambi, R., \& Navarra, P. (2004). Is knowledge power? Knowledge flows, subsidiary power and rent-seeking within MNCs. Journal of International Business Studies, 35(5), 385406.

Mudambi, R., Pedersen, T., \& Andersson, U. (2014). How subsidiaries gain power in multinational corporations. Journal of World Business, 49(1), 101-113.

Myers, R. H., \& Myers, R. H. (1990). Classical and modern regression with applications (Vol. 2). Belmont, CA: Duxbury press.

Najafi-Tavani, Z., Giroud, A., \& Sinkovics, R. R. (2012). Mediating effects in reverse knowledge transfer processes. Management International Review, 52(3), 461-488.

Najafi-Tavani, Z., Zaefarian, G., Naudé, P., \& Giroud, A. (2015). Reverse knowledge transfer and subsidiary power. Industrial Marketing Management, 48(2015), 103-110.

Nebus, J. (2006). Building Collegial Information Networks: A theory of advice networks generation. Academy of Management Review, 31(3), 615-637.

Nguyen, N. P., Ngo, L. V., Bucic, T., \& Phong, N. D. (2018). Cross-functional knowledge sharing, coordination and firm performance: The role of cross-functional competition. Industrial Marketing Management, 71(2018), 123-134.

Nobel, R., \& Birkinshaw, J. (1998). Innovation in multinational corporations : Control and communication patterns in international R \& D operations. Strategic Entrepreneurship 
Journal, 19(5), 479-496.

Nonaka, I., \& Takeuchi, H., 1995. The knowledge-creating company. New York: Oxford University Press, New York.

Noorderhaven, N., \& Harzing, A.-W. (2009). Knowledge-sharing and social interaction within MNEs. Journal of International Business Studies, 40(5), 719-741.

Ott, D. L., \& Michailova, S. (2018). Cultural intelligence: A review and new research avenues. International Journal of Management Reviews, 20(1), 99-119.

Pallant, J. (2010). SPSS survival manual: A step by step guide to data analysis using the SPSS program. 4th Edition, McGraw Hill, New York.

Pallant, J., \& Manual, S. S. (2010). A step by step guide to data analysis using SPSS. Berkshire UK: McGraw-Hill Education.

Patriotta, G., Castellano, A., \& Wright, M. (2013). Coordinating knowledge transfer: Global managers as higher-level intermediaries. Journal of World Business, 48(4), 515-526.

Park, J., Chae, H., \& Choi, J.N. (2017). The need for status as a hidden motive of knowledgesharing behavior: An application of costly signaling theory. Human Performance 30(1), 21-37.

Pedersen, T., Petersen, B., \& Sharma, D. (2003). Knowledge transfer performance of multinational companies. Management International Review, 3(Special Issue), 69-90.

Pellegrini, E. K., Scandura, T. A., \& Jayaraman, V. (2010). Cross-cultural generalizability of paternalistic leadership: An expansion of leader-member exchange theory. Group \& Organization Management, 35(4), 391-420.

Peltokorpi, V. (2015). Corporate language proficiency and reverse knowledge transfer in multinational corporations: Interactive effects of communication media richness and commitment to headquarters. Journal of International Management, 21(1), 49-62.

Peltokorpi, V., \& Yamao, S. (2017). Corporate language proficiency in reverse knowledge transfer: A moderated mediation model of shared vision and communication frequency. Journal of World Business, 52(3), 404-416.

Peteraf, M. A. (1993). The Cornerstones of competitive advantage: A resource-based view. Journal of Chemical Information and Modeling, 53(9), 1689-1699.

Pfeffer, J., \& Salancik, G. R. (1978). The external control of organizations: A resource dependence perspective. Stanford University Press.

Pfeffer, J. (1981). Power in organizations. Marshfield, MA: Pitman.

Pfeffer, J., \& Fong, C. T. (2005). Building organization theory from first principles: The selfenhancement motive and understanding power and influence. Organization Science, 16(4), 372-388.

Pfeffer, J., \& Salancik, G. R. (1974). Organizational decision making as a political process: The case of a university budget. Administrative Science Quarterly, 19(2), 135-151.

Phene, A., \& Almeida, P. (2008). Innovation in multinational subsidiaries: The role of knowledge assimilation and subsidiary capabilities. Journal of International Business Studies, 39(5), 901-919.

Phillips, D. C., \& Burbules, N. C. (2000). Postpositivism and Educational Research. Lanham, Md: Rowman \& Littlefield Publishers.

Podolny, J. M. (2001). Networks as the Pipes and Prisms of the Market. American Journal of Sociology, 107(1), 33-60.

Podsakoff, P. M., MacKenzie, S. B., Lee, J.-Y., \& Podsakoff, N. P. (2003). Common method biases in behavioral research: a critical review of the literature and recommended remedies. Journal of Applied Psychology, 88(5), 879-903.

Polyani, M. (1966). The Tacit Dimension. London: Routledge.

Pothukuchi, V., Damanpour, F., Choi, J., Chen, C. C., \& Park, S. H. (2002). National and organizational culture differences and international joint venture performance. Journal of 
International Business Studies, 33(2), 243-265.

Preacher, K. J., \& Kelley, K. (2011). Effect size measures for mediation models: quantitative strategies for communicating indirect effects. Psychological Methods, 16(2), 93.

Preacher, K. J., Rucker, D. D., \& Hayes, A. F. (2007). Addressing moderated mediation hypotheses: Theory, methods, and prescriptions. Multivariate Behavioral Research, 42(1), 185-227.

Provan, K. G. (1980). Recognizing, measuring, and interpreting the potential/enacted power distinction in organizational research. The Academy of Management Review, 5(4), 549559.

Quigley, N. R., Tesluk, P. E., Locke, E. A., \& Bartol, K. M. (2007). A multilevel investigation of the motivational mechanisms underlying knowledge sharing and performance. Organization Science, 18(1), 71-88.

Raab, K. J., Ambos, B., \& Tallman, S. (2014). Strong or invisible hands? - Managerial involvement in the knowledge sharing process of globally dispersed knowledge groups. Journal of World Business, 49(1), 32-41.

Rabinovich, A., Morton, T. A., Postmes, T., \& Verplanken, B. (2012). Collective self and individual choice: The effects of inter-group comparative context on environmental values and behaviour. British Journal of Social Psychology, 51(4), 551-569.

Raudenbush, S. W., \& Bryk, A. S. (2002). Hierarchical Linear Models: Applications and Data Analysis Methods (2nd ed.). Newbury Park, CA: Sage publications.

Reade, C. (2001a). Antecedents of organizational identification in multinational corporations: fostering psychological attachment to the local subsidiary and the global organization. The International Journal of Human Resource Management, 12(8), 1269-1291.

Reade, C. (2001b). Dual identification in multinational corporations: local managers and their psychological attachment to the subsidiary versus the global organization. The International Journal of Human Resource Management, 12(8), 1269-1291.

Reagans, R., \& Mcevily, B. (2003). Network structure and knowledge transfer: The effects of cohesion and range. Administrative Science Quarterly, 48(2), 240-267.

Rehg, M. T., Gundlach, M. J., \& Grigorian, R. A. (2012). Examining the influence of crosscultural training on cultural intelligence and specific self-efficacy. Cross Cultural Management: An International Journal, 19(2), 215-232.

Reichard, R. J., Serrano, S. A., Condren, M., Wilder, N., Dollwet, M., \& Wang, W. (2015). Engagement in cultural trigger events in the development of cultural competence. Academy of Management Learning \& Education, 14(4), 461-481.

Reiche, B. S., Harzing, A.-W., \& Pudelko, M. (2015). Why and how does shared language affect subsidiary knowledge inflows? A social identity perspective. Journal of International Business Studies, 46(5), 528-551.

Reinholt, M., Pedersen, T., Foss, N.J., 2011. Why a central network position isn't enough: The role of motivation and ability for knowledge sharing in employee networks. Academy of Management Journal 54(6), 1277-1297.

Richter, A. W., West, M. A., Van Dick, R., \& Dawson, J. F. (2006). Boundary spanners' identification, intergroup contact, and effective intergroup relations. Academy of Management Journal, 49(6), 1252-1269.

Roberts, L. M. (2005). Changing faces: Professional image construction in diverse organizational settings. Academy of Management Review, 30(4), 685-711.

Rockstuhl, T., Seiler, S., Ang, S., Van Dyne, L., \& Annen, H. (2011). Beyond general intelligence (IQ) and emotional intelligence (EQ): The role of cultural intelligence (CQ) on cross-border leadership effectiveness in a globalized world. Journal of Social Issues, 67(4), 825-840.

Rossiter, J. R. (2002). The C-OAR-SE procedure for scale development in 
marketing. International Journal of Research in Marketing, 19(4), 305-335.

Rucker, D. D., \& Galinsky, A. D. (2008). Desire to Acquire: Powerlessness and compensatory consumption. Journal of Consumer Research, 35(2), 257-267.

Rucker, D. D., Preacher, K. J., Tormala, Z. L., \& Petty, R. E. (2011). Mediation analysis in social psychology: Current practices and new recommendations. Social and Personality Psychology Compass, 5(6), 359-371.

Saunders, M., Lewis, P., \& Thornhill, A. (2009). Research Methods for Business Students. Essex. Financial Times/Prentice Hall.

Sachdev, I., \& Bourhis, R. Y. (1991). Power and status differentials in minority and majority group relations. European Journal of Social Psychology, 21(1), 1-24.

Sachdev, S. B., \& Verma, H. V. (2004). Relative importance of service quality dimensions: A multi-sectoral study. Journal of Services Research, 4(1), 93-116.

Salancik, G. R., \& Pfeffer, J. (1974). The bases and use of power in organizational decision making: The case of a university. Administrative Science Quarterly, 19(4), 453-473.

Salancik, G. R., \& Pfeffer, J. (1977). Who gets power-and how they hold on to it: A strategiccontingency model of power. Organizational Dynamics, 5(3), 3-21.

Sax, L. J., Gilmartin, S. K., \& Bryant, A. N. (2003). Assessing response rates and nonresponse bias in web and paper surveys. Research in Higher Education, 44(4), 409-432.

Scandura, T. A., \& Williams, E. a. (2000). Research methodology in management: Current practices , trends , and implications for future research. Academy of Management Journal, $43(6), 1248-1264$.

Schein, V. F. (1977). Individual power and political behaviors in organizations: An inadequately explored reality. Academy of Management Review, 2(1), 64-72.

Schlegelmilch, B. B., \& Chini, T. C. (2003). Knowledge transfer between marketing functions in multinational companies: A conceptual model. International Business Review, 12(2), 215-232.

Schlenker, B. R., \& Weigold, M. F. (1992). Interpersonal processes involving impression regulation and management. Annual Review of Psychology, 43(1), 133-168.

Schrader, S. (1991). Informal technology transfer between firms: Cooperation through information trading. Research Policy, 20(2), 153-170.

Schulz, M. (2003). Pathways of relevance: Exploring inflows of knowledge into subunits of multinational. Organization Science, 14(4), 440-459.

See, K. E., Morrison, E. W., Rothman, N. B., \& Soll, J. B. (2011). The detrimental effects of power on confidence, advice taking, and accuracy. Organizational Behavior and Human Decision Processes, 116(2), 272-285.

Sekaran, U., \& Bougie, R. (2016). Research methods for business: A skill building approach. John Wiley \& Sons.

Sharma, R. R. (2019). Cultural intelligence and institutional success: The mediating role of relationship quality. Journal of International Management, 25(3), 100665.

Shook, C. L., Ketchen Jr, D. J., Hult, G. T. M., \& Kacmar, K. M. (2004). An assessment of the use of structural equation modeling in strategic management research. Strategic management journal, 25(4), 397-404.

Shrout, P. E., \& Bolger, N. (2002). Mediation in experimental and non-experimental studies: new procedures and recommendations. Psychological methods, 7(4), 422.

Simon, H. A. (1991). Bounded rationality and organizational learning. Organization Science, 2(1), 125-134.

Sivanathan, N., Pillutla, M. M., \& Murnighan, J. K. (2008). Power gained, power lost. Organizational Behavior and Human Decision Processes, 105(2), 135-146.

Smale, A., Björkman, I., Ehrnrooth, M., John, S., Mäkelä, K., \& Sumelius, J. (2015). Dual values-based organizational identification in MNC subsidiaries: A multilevel study. 
Journal of International Business Studies, 46(7), 761-783.

Smidts, A., Pruyn, A., \& van Riel, C.B.M. (2001). The impact of employee communication and perceived external prestige on organizational identification. Academy of Management Journal 44(5), 1051-1062.

Smith, P. B., Peterson, M. F., Ahmad, A. H., Akande, D., Andersen, J. A., Ayestaran, S., \& Ekelund, B. (2005). Demographic effects on the use of vertical sources of guidance by managers in widely differing cultural contexts. International Journal of Cross Cultural Management, 5(1), 5-26.

Smith, P. K., Smallman, R., \& Rucker, D. D. (2016). Power and categorization: Power increases the number and abstractness of categories. Social Psychological and Personality Science, 7(3), 281-289.

Sobel, M. E. (1986). Some new results on indirect effects and their standard errors in covariance structure models. Sociological Methodology, 16(1986), 159-186.

Sparrowe, R. T., \& Mayer, K. J. (2011). Publishing in AMJ - Part 4: Grounding hypotheses. (hypotheses grounded in the theory section of any paper submitted to the Academy of Management Journal)(Editorial). Academy of Management Journal, 54(6), 1098-1102.

Sposito, V. A., Hand, M. L., \& Skarpness, B. (1983). On the efficiency of using the sample kurtosis in selecting optimal lp estimators. Communications in Statistics-simulation and Computation, 12(3), 265-272.

Steinel, W., Utz, S., Koning, L., 2010. The good, the bad and the ugly thing to do when sharing information: Revealing, concealing and lying depend on social motivation, distribution and importance of information. Organizational Behavior and Human Decision Processes 113(2), 85-96.

Stenius, M., Haukkala, A., Hankonen, N., \& Ravaja, N. (2017). What motivates experts to share? A prospective test of the model of knowledge-sharing motivation. Human Resource Management, 56(6), 871-885

Sternberg, R. J. (1997). The concept of intelligence and its role in lifelong learning and success. American Psychologist, 52(10), 1030.

Szulanski, G. (1996). Exploring internal stickiness: Impediments to the transfer of best practice within the Firm. Strategic Management Journal, 17(Winter Special Issue), 27-43.

Szulanski, G. (2000). The process of knowledge transfer: A diachronic analysis of stickiness. Organizational Behavior and Human Decision Processes, 82(1), 9-27.

Szulanski, G. (2003). Sticky knowledge. Barriers to Knowing in the Firm, London: SAGE Publications.

Tabachnick, B. G., \& Fidell, L. S. (2007). Using multivariate statistics (5th ed.). Boston, MA, USA: Allyn \& Bacon.

Tajfel, H. (1978). Differentiation between social groups : studies in the social psychology of intergroup relations. New York, NY: Academic Press.

Tajfel, H. , \& Turner, J.C. (1979). An integrative theory of intergroup conflict. In W.G. Austin $\&$ S. Worchel (Eds.), The Social Psychology of Intergroup Relations (pp. 3347). Monterey, CA: Brooks/Cole.

Teigland, R., Fey, C. F., \& Birkinshaw, J. (2000). Knowledge dissemination in global R \& D operations: An empirical study of multinationals in the High Technology Electronics Industry. Management International Review, 1(Special Issue), 49-77.

Terry, D. J., \& Hogg, M. A. (1996). Group norms and the attitude-behavior relationship: A role for group identification. Society for Personality and Social Psychology, 22(8), 776-793.

Terry, D. J., Hogg, M. a, \& White, K. M. (1999). The theory of planned behaviour: self-identity, social identity and group norms. The British Journal of Social Psychology / The British Psychological Society, 38(3), 225-244.

Thomas, D. C., Elron, E., Stahl, G., Ekelund, B. Z., Ravlin, E. C., Cerdin, J. L., ... \& Maznevski, 
M. (2008). Cultural intelligence: Domain and assessment. International Journal of Cross Cultural Management, 8(2), 123-143.

Thomas, D. C., Liao, Y., Aycan, Z., Cerdin, J. L., Pekerti, A. A., Ravlin, E. C., ... \& Moeller, M. (2015). Cultural intelligence: A theory-based, short form measure. Journal of International Business Studies, 46(9), 1099-1118.

Tippmann, E., Scott, P. S., Reilly, M., \& O’Brien, D. (2018). Subsidiary coopetition competence: Navigating subsidiary evolution in the multinational corporation. Journal of World Business, 53(4), 540-554.

Toh, S. M., \& Srinivas, E. S. (2012). Perceptions of task cohesiveness and organizational support increase trust and information sharing between host country nationals and expatriate coworkers in Oman. Journal of World Business, 47(4), 696-705.

Tolman, E. C. (1943). Identification and the post-war world. The Journal of Abnormal and Social Psychology, 38(2), 141-148.

Tortoriello, M., Reagans, R., \& McEvily, B. (2012). Bridging the knowledge gap: The influence of strong ties, network cohesion, and network range on the transfer of knowledge between organizational units. Organization Science, 23(4), 1024-1039.

Tost, L. P., Gino, F., \& Larrick, R. P. (2012). Power, competitiveness, and advice taking: Why the powerful don't listen. Organizational Behavior and Human Decision Processes, $117(1), 53-65$.

Tsai, W. (2001). Knowledge transfer in intraorganizational networks: Effects of network position and absorptive capacity on business unit innovation and performance. Academy of Management Journal 44(5), 996-1004.

Tsai, W., 2002. Social structure of "coopetition" within a multiunit organization: Coordination, competition, and intraorganizational knowledge sharing. Organization Science 13(2), 179-190.

Tsai, W., \& Ghoshal, S. (1998). Social capital and value creation: The role of intrafirm networks. The Academy of Management Journal, 41(4), 464-476.

Tsang, E. W. (1999). The knowledge transfer and learning aspects of international HRM: an empirical study of Singapore MNCs. International Business Review, 8(5-6), 591-609.

Tseng, C.-H. (2015). Determinants of MNC's knowledge inflows to subsidiaries: A perspective on internalization advantages. Management International Review, 55(1), 119-150.

Turner, J. C. (1975). Social comparison and social identity: Some prospects for intergroup behavior. European Journal of Social Psychology, 5(1), 5-34.

Turner, R. A., \& Schabram, K. F. (2012). The bases of power revisited: An interpersonal perceptions perspective. Journal of Organizational Psychology, 12(1), 9-18.

Uzzi, B. (1997). Social structure and competition in interfirm networks: The Paradox of embeddedness. Administrative Science Quarterly, 42(1), 35-67.

van Knippenberg, D., \& van Schie, E. C. M. (2000). Foci and correlates of organizational identification. Journal of Occupational and Organizational Psychology, 73(2), 137-147.

Viswesvaran, C., Barrick, M. R., \& Ones, D. S. (1993). How definitive are conclusions based on survey data: Estimating robustness to nonresponse. Personnel Psychology, 46(3), 551567.

Vlajčić, D., Caputo, A., Marzi, G., \& Dabić, M. (2019). Expatriates managers' cultural intelligence as promoter of knowledge transfer in multinational companies. Journal of Business Research, 94, 367-377.

Vora, D., \& Kostova, T. (2007). A model of dual organizational identification in the context of the multinational enterprise. Journal of Organizational Behavior, 28(3), 327-350.

Vora, D., Kostova, T., \& Roth, K. (2007). Roles of subsidiary managers in multinational corporations: The effect of dual organizational identification. Management International Review, 47(4), 595-620. 
Wagner, S. M., Rau, C., \& Lindemann, E. (2010). Multiple informant methodology: A critical review and recommendations. Sociological Methods \& Research, 38(4), 582-618.

Wahyuni, D. (2012). The research design maze: Understanding paradigms, cases, methods and methodologies. Journal of Applied Management Accounting Research, 10(1), 69-80.

Waldman, D. A., De Luque, M. S., Washburn, N., House, R. J., Adetoun, B., Barrasa, A., ... \& Dorfman, P. (2006). Cultural and leadership predictors of corporate social responsibility values of top management: A GLOBE study of 15 countries. Journal of International Business Studies, 37(6), 823-837.

Wang, P., Tong, T. W., \& Koh, C. P. (2004). An integrated model of knowledge transfer from MNC parent to China subsidiary. Journal of World Business, 39(2), 168-182.

Wang, S., \& Noe, R. A. (2010). Knowledge sharing: A review and directions for future research. Human Resource Management Review, 20(2), 115-131.

Wang, S., Noe, R. A., \& Wang, Z.-M. (2014). Motivating knowledge sharing in knowledge management systems: A Quasi-Field experiment. Journal of Management, 40(4), 9781009.

Wang, S., Tong, T. W., Chen, G., \& Kim, H. (2009). Expatriate utilization and foreign direct investment performance: The mediating role of knowledge transfer. Journal of Management, 35(5), 1181-1206.

Weber, M. (1947). Theory of Social and Economic Organizations. New York: Oxford University Press.

Welch, D. E., \& Welch, L. S. (2008). The importance of language in international knowledge transfer. Management International Review, 48(3), 339-360.

Wernerfelt, B. (1984). The resource-based view of the firm. Strategic Management Journal, 3(June 1982), 171-180.

White, H. (1980). A heteroskedasticity-consistent covariance matrix estimator and a direct test for heteroskedasticity. Econometrica, 48(4), 817-838.

Whittle, A., Mueller, F., Gilchrist, A., \& Lenney, P. (2016). Sensemaking, sense-censoring and strategic inaction: The discursive enactment of power and politics in a multinational corporation. Organization Studies, 1-29.

Williamson, O. (1985). The economic institutions of capitalism: Firms, markets, relational contracting. New York : London: Free Press, Collier Macmillan.

Williams, L. J., Hartman, N., \& Cavazotte, F. (2010). Method variance and marker variables: A review and comprehensive CFA marker technique. Organizational Research Methods, 13(3), 477-514.

Williams, M. N., Grajales, C. A. G., \& Kurkiewicz, D. (2013). Assumptions of Multiple Regression: Correcting two misconceptions. Practical Assessment, Research \& Evaluation, 18(11), 2.

Wong, S.-S., Ho, V. T., \& Lee, C. H. (2008). A power perspective to interunit knowledge transfer: Linking knowledge attributes to unit power and the transfer of knowledge. Journal of Management, 34(1), 127-150.

Wood, E. D., \& St. Peters, H. Y. (2014). Short-term cross-cultural study tours: impact on cultural intelligence. The International Journal of Human Resource Management, 25(4), 558-570.

Wood, R. E., Goodman, J. S., Beckmann, N., \& Cook, A. (2008). Mediation testing in management research: A review and proposals. Organizational Research Methods, 11(2), 270-295.

Zander, U., \& Kogut, B. (1995). Knowledge and the speed of the transfer and imitation of organizational capabilities: an empirical test. Organization Science, 6(1), 76-92.

Zhao, H., \& Luo, Y. (2005). Antecedents of knowledge sharing with peer subsidiaries in other countries: A perspective from subsidiary managers in a foreign emerging market. 
Management International Review, 45(1), 71-97

Zhao, Z.J., \& Anand, J. (2013). Beyond boundary spanners: The 'collective bridge' as an efficient interunit structure for transferring collective knowledge. Strategic Management Journal 34(13), 1513-1530.

Zhou, Fey, \& Yildiz. (2018). Fostering integration through HRM practices: An empirical examination of absorptive capacity and knowledge transfer in cross-border M\&As. Journal of World Business, 100947. 